University of Patras

Faculty of Medicine

National Technical University of Athens

Faculty of Electrical and Computer Engineering

National Technical University of Athens

Faculty of Mechanical Engineering
Interdepartmental Program

of Postgraduate Studies

in Biomedical Engineering

\title{
ADVANCED THREE DIMENSIONAL DIGITAL TOMOSYNTHESIS STUDIES FOR BREAST IMAGING
}

\author{
PH.D. THESIS
}

University of Patras

\author{
Anthi Malliori
}

Faculty of Medicine

Department of Medical Physics

265 00, Rio-Patras, GREECE

Prof. Nicolas Pallikarakis 


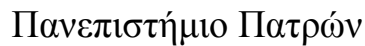

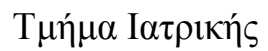

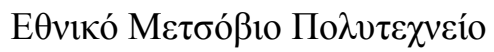

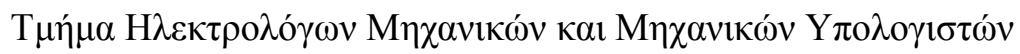

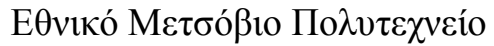

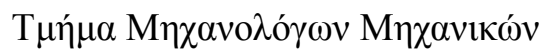

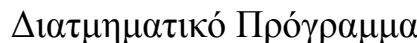

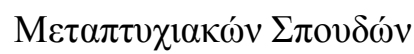

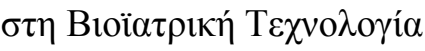

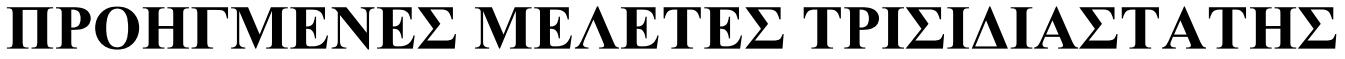

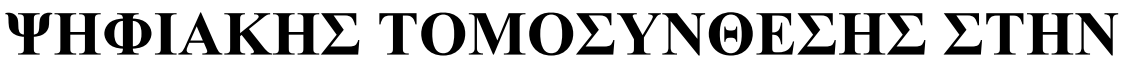

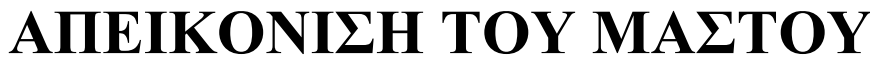

\author{
$\Delta$ I $\Delta$ AKTOPIKH $\Delta I A T P I B H$
}

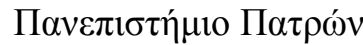

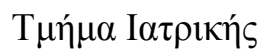

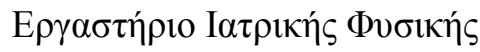

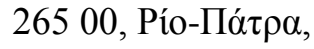

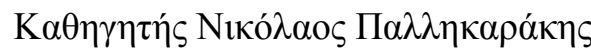

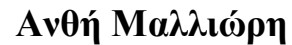

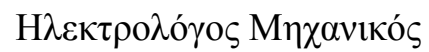

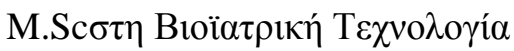

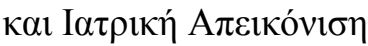


Three Members Advisory Committee

Professor Nicolas Pallikarakis,

Professor Konstantina Nikita,

Associate Professor Ivan Buliev,

Seven Members Examination Committee

Professor Nicolas Pallikarakis,

Professor Konstantina Nikita,

Associate Professor Ivan Buliev,

Professor Dimitrios Kardamakis,

Professor Dimitrios Koutsouris,

Associate Professor Eleni Costaridou,

Assistant Professor Vasiliki Bravou,
Main Supervisor

Member of Advisory Committee

Member of Advisory Committee

Main Supervisor

Member of Advisory Committee

Member of Advisory Committee Member of Examination Committee Member of Examination Committee Member of Examination Committee Member of Examination Committee 


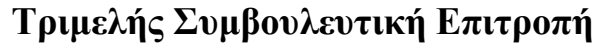

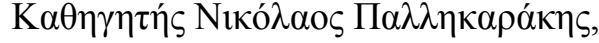

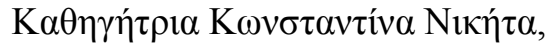

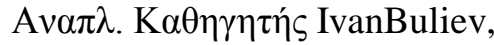

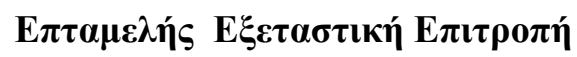

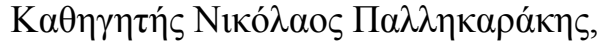

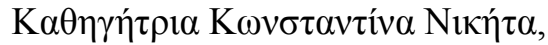

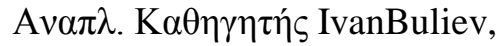

$\mathrm{K} \alpha \theta \eta \gamma \eta \tau \dot{\varsigma} \varsigma \Delta \eta \mu \eta \dot{\tau} \rho 10 \varsigma \mathrm{K} \alpha \rho \delta \alpha \mu \alpha \dot{\alpha} \kappa \eta$,

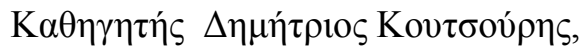

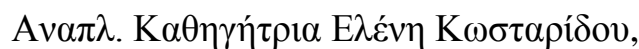

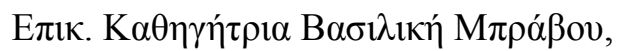

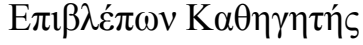

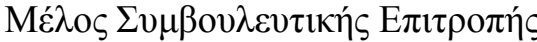

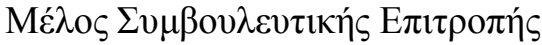

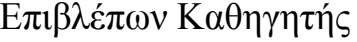

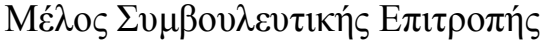

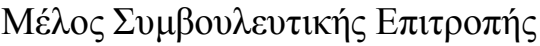

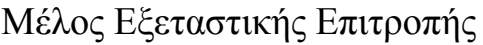

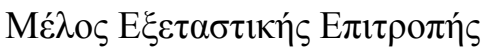

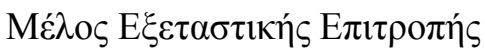

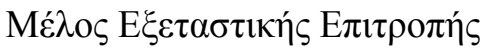




\section{ACKNOWLEDGEMENTS}

I wish to express my sincere gratitude to my supervisor, Professor Nicolas Pallikarakis for trusting me with the assignment of this project and for the opportunity to work in a team with great expertise and long research activity in the field of tomosynthesis. I am grateful for his scientific guidance and support throughout my entire thesis.

I am also grateful to Dr. Kristina Bliznakova for introducing me to this field and for sharing with me her knowledge and experience. I am deeply thankful for her continuous guidance, substantial contribution, the extensive exchange of ideas and the close collaboration we had from the very beginning of this project.

I am would like to express my great appreciation to Prof. Konstantina Nikita and Prof. Ivan Buliev, members of my advisory committee, for their support and contribution in carrying out my thesis.

I would like to thank Prof. Robert Speller, Prof. Julie Horrocks, Dr. Juliana Tromba and Dr. Luigi Rigon for their valuable contribution and advice during the experiments performed at Elettra Synchrotron facilities in Trieste and the analysis of the results.

I am also grateful to Prof. Hilde Bosmans and Dr. Nick Marshall for sharing knowledge with me and giving me the chance to carry out experimental work during the time I spent at the University Hospital in Leuven, Belgium.

I am thankful to Dr. Zhivko Bliznakov and Dr. Zacharias Kamarianakis for their valuable help and exchange of ideas.

Many thanks to all the members of the Biomedical Technology Unit, being not only colleagues but also close friends, for the excellent collaboration and nice moments we shared all these years.

Lastly and most of all I am grateful to my family for their constant and precious support in every possible way.

The current thesis has been supported by the Research Funding Program: Heracleitus II. Investing in knowledge society through the European Social Fund. 


\section{CONTENTS}

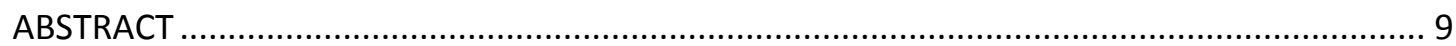

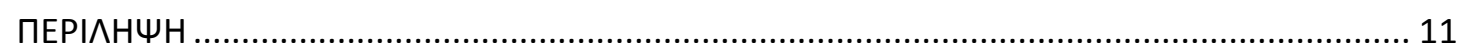

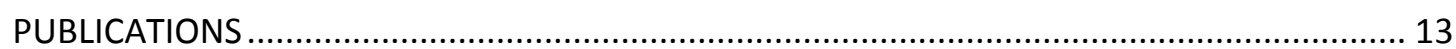

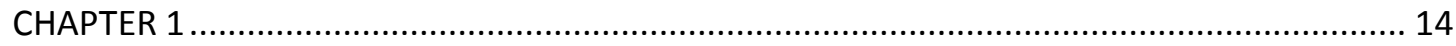

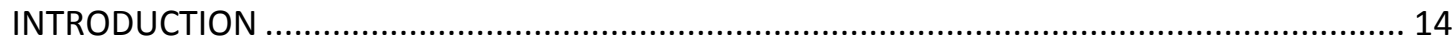

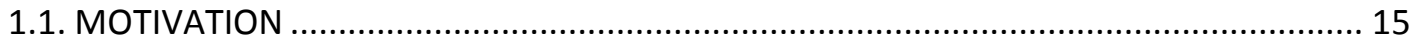

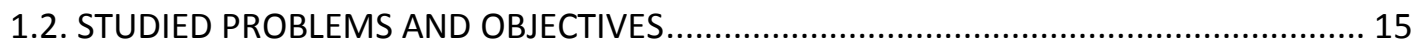

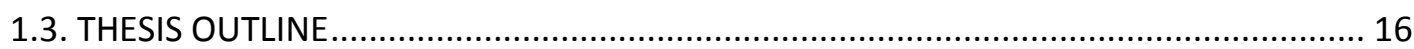

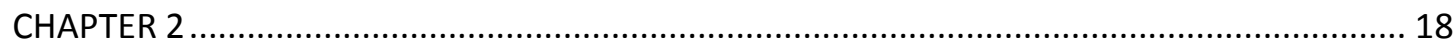

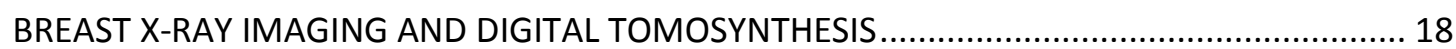

2.1. BREAST ANATOMY AND CONVENTIONAL MAMMOGRAPHY....................................... 19

2.1.1. Anatomy of the normal breast ............................................................19

2.1.2. Mammography ...................................................................................................20 20

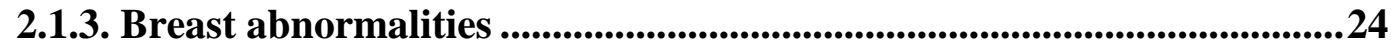

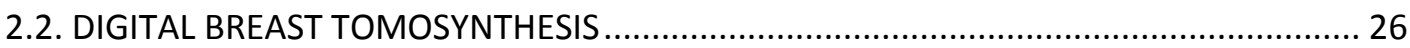

2.2.1. Principles of Tomosynthesis ..............................................................26

2.2.2. Systems and acquisition technologies ........................................................28

2.2.3. Reconstruction algorithms ..............................................................32

2.2.4. Noise and filters ...........................................................................................34

2.2.5. Evaluation metrics...................................................................................................36

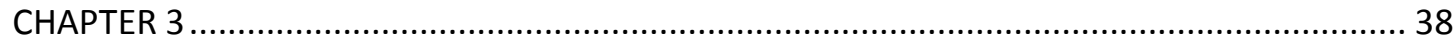

SYNCHROTRON RADIATION AND APPLICATIONS IN BREAST IMAGING .................................. 38

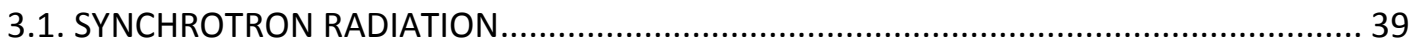

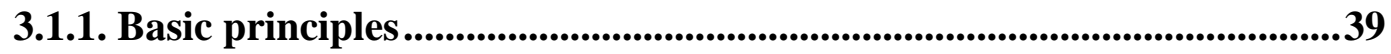

3.1.2. Synchrotron facilities ..........................................................................41

3.2. MONOCHROMATIC AND POLYCHROMATIC X-RAYS FOR MEDICAL IMAGING:

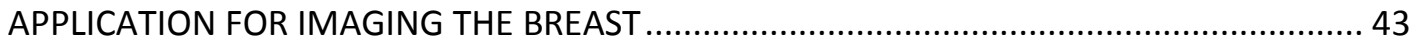

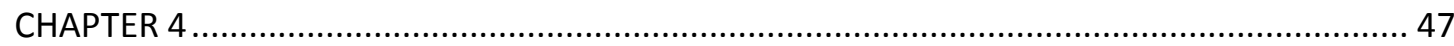

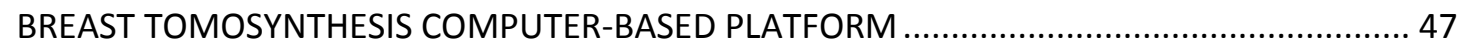

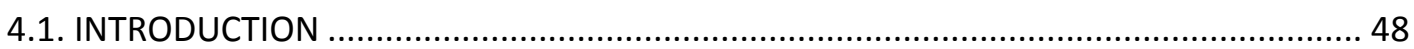

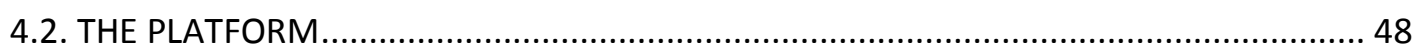

4.2.1. Design of the computer-based tomosynthesis platform ...........................48

4.2.2. Graphical User Interface ................................................................................50 
4.3. BASIC PRINCIPLES AND MATHEMATICAL DESCRIPTION OF THE RECONSTUCTION

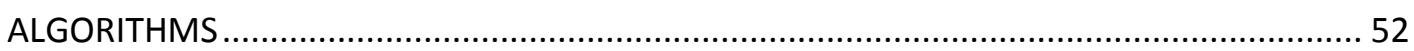

4.3.1. The Multiple Projection Algorithm ..................................................................52

4.3.2. The Backprojection algorithm ................................................................56

4.4. INITIAL TESTING USING A SOFTWARE BREAST PHANTOM........................................ 58

4.4.1. Phantom and acquisition parameters............................................................58

4.4.2. Results and conclusions .......................................................................66

4.5. INITIAL TESTING USING A HARDWARE BREAST PHANTOM: APPLICATION OF NON-

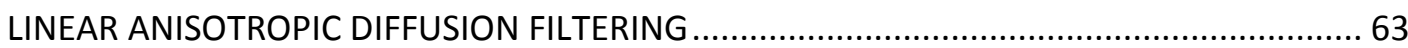

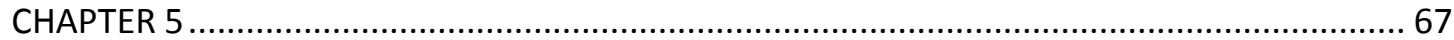

EVALUATION OF THE MPA ADAPTED FOR BREAST TOMOSYNTHESIS WITH PARTIAL ISOCENTRIC ROTATION: A STRUCTURED PHANTOM STUDY IN A CLINICAL SETTING ...............67

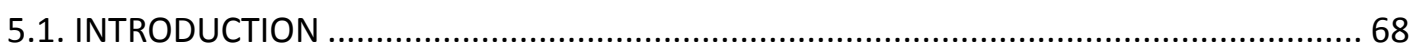

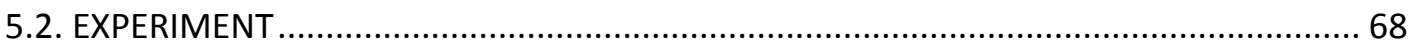

5.2.1. Breast phantom and image acquisition ......................................................68

5.2.2. Reconstruction methods and evaluation ...................................................... 71

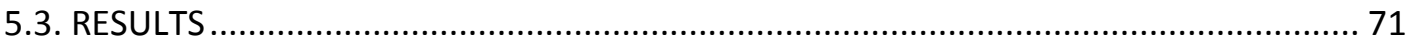

5.3.1. Comparison of MPA versus BP .................................................................... 72

5.3.2. Comparison of tomosynthesis slices with $2 \mathrm{D}$ projection mammography

5.3.3. Evaluation of reconstruction algorithms as a function of phantom

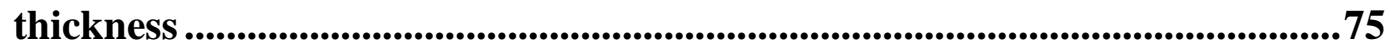

5.3.4. Influence of the phantom configuration..............................................77

5.3.5. Influence of the object size...................................................................................78

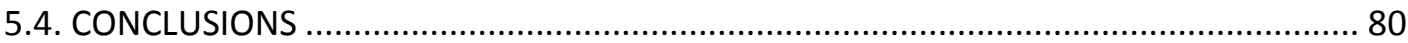

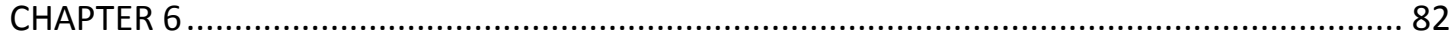

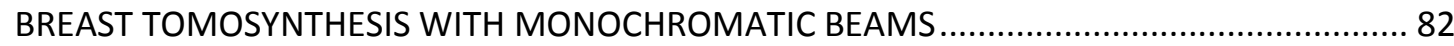

6.1. SIMULATION STUDIES: OPTIMISATION OF ACQUISITION PARAMETERS USING A

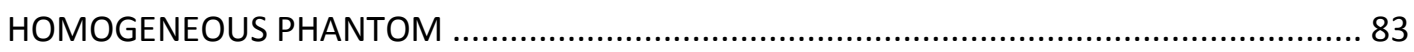

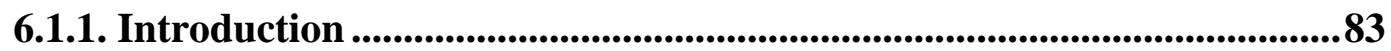

6.1.2. Phantom and acquisition scenarios ............................................................8 84

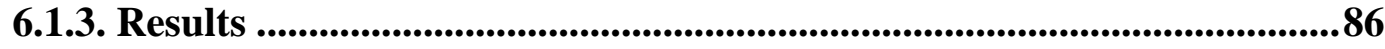

6.2. EXPERIMENTAL WORK AT ELLETRA SYNCHROTRON FACILITIES: OPTIMISATION OF ACQUISITION PARAMETERS USING A HETEROGENEOUS PHANTOM .................................. 89

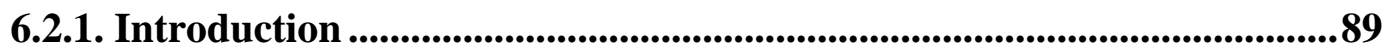


6.2.2. Experimental Setup and methods ....................................................90

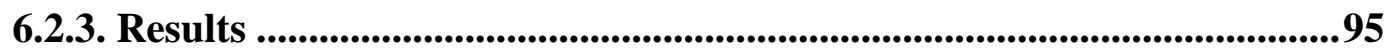

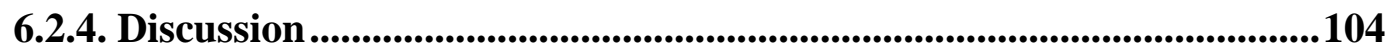

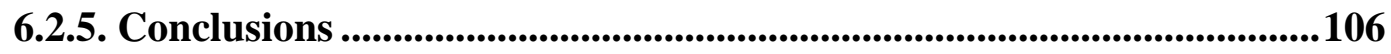

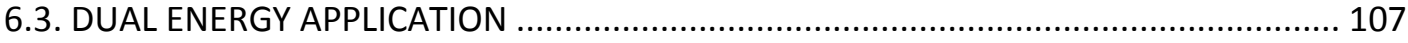

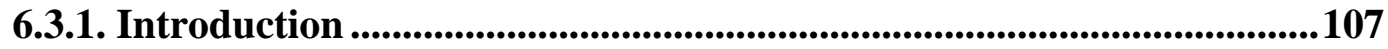

6.3.2. Materials and Methods .........................................................................107

6.3.3. Results and conclusions ............................................................................108

6.4. MONOENERGETIC vs POLYENERGETIC: EXPERIMENTAL STUDIES ............................. 110

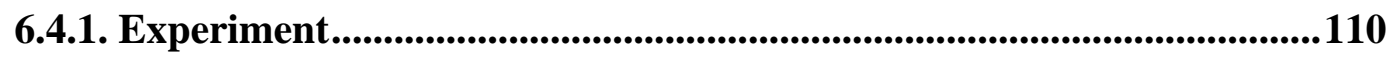

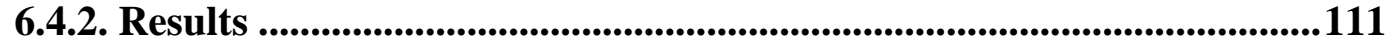

6.5. MONOENERGETIC vS POLYENERGETIC: SIMULATION STUDIES .............................. 113

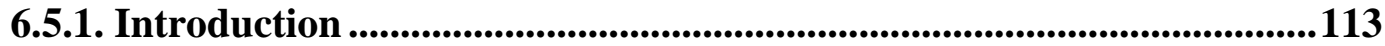

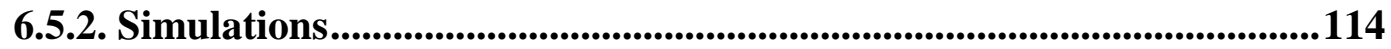

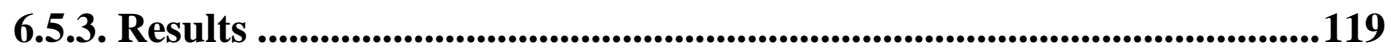

6.5.4. Conclusions ........................................................................................128

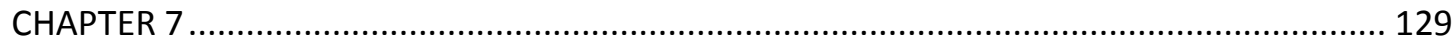

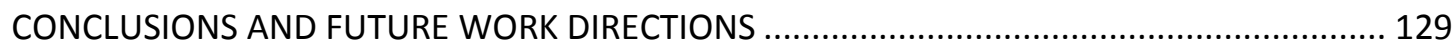

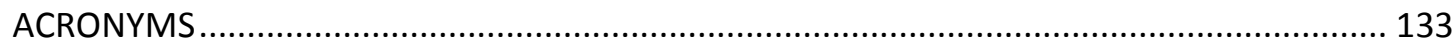

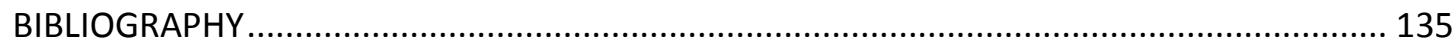


The current thesis is focused on the study of tomosynthesis techniques applied on breast imaging, in order to improve the detection of breast lesions. Breast Tomosynthesis (BT) is a pseudo-three-dimensional (3D) x-ray imaging technique that provides reconstructed tomographic images from a set of angular projections taken in a limited arc around the breast, with dose levels similar to those of a two-view conventional mammography. Simulation studies and clinical trials suggest that BT is very useful for imaging the breast in an attempt to optimize the detection and characterization of lesions particularly in dense breasts and has the potential to reduce the recall rate. Reconstruction algorithms and acquisition parameters are critical for the quality of reconstructed slices.

The aim of this research is to explore tomosynthesis modalities for breast imaging and evaluate them against existing mammographic techniques as well as to investigate the effect of reconstruction algorithms and acquisition parameters on the image quality of tomosynthetic slices. A specific aim and innovation of the study was to demonstrate the feasibility of combining BT and monochromatic radiation for 3D breast imaging, an approach that had not been studied thoroughly yet.

For the purposes of this study a computer-based platform has been developed in Matlab incorporating reconstruction algorithms and filtering techniques for BT applications. It is fully parameterized and has a modular architecture for easy addition of new algorithms. Simulations studies with the XRayImaging Simulator and experimental work at ELETTRA Synchrotron facilities in Trieste, Italy, have been performed using software and complex hardware phantoms, of realistic shape and size, consisting of materials mimicking the breast tissue. The work has been carried out in comparison to conventional BT and mammography and demonstrates the feasibility of the studied new technique and the potential advantages of using BT with synchrotron modality for the detection of breast low- and high-contrast breast lesions such as masses and microcalcifications $(\mu \mathrm{Cs})$.

Evaluations of both simulation and experimental tomograms demonstrated superior visibility of all reconstructed features using appropriately optimized filtered algorithms. Moreover, image quality and evaluation metrics are improved with extending the acquisition length for the masses. The visualization of $\mu$ Cs was found less sensitive to this parameter due to their high inherent contrast. Breast tomosynthesis shows advantages in visualizing features of small size within phantoms of increased thickness and especially in bringing into focus and localizing low-contrast masses hidden in a highly heterogeneous background with 
superimposed structures. Monochromatic beams can result in better tissue differentiation and in combination with BT can lead to improvement of features' visibility, better detail and higher contrast. Monochromatic BT provided improved image quality at lower incident exposures, compared to conventional mammography, concerning mass detection and visibility of borders, which is important for their characterization, especially when they are spiculated. Overall it has been proved that while reducing the radiation dose, monochromatic beams combined with BT, result in an improvement of image quality. These findings are encouraging for the development of a tomosynthesis system based on monochromatic beams. 


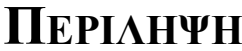

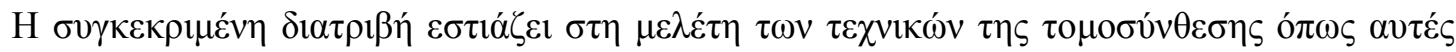

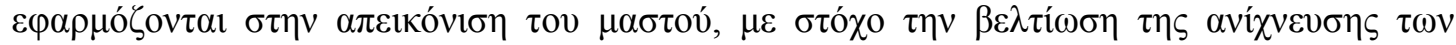

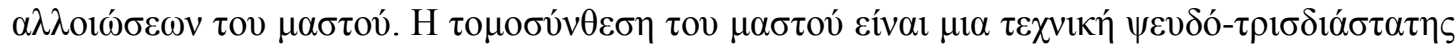

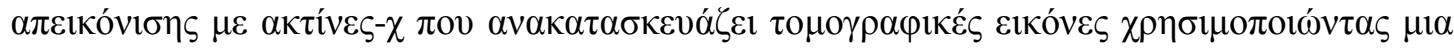

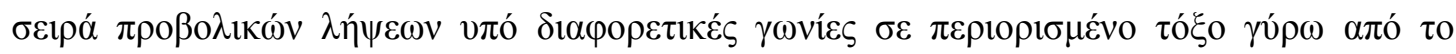

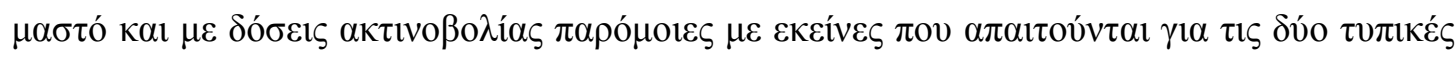

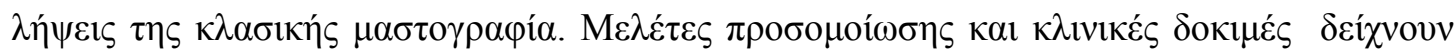

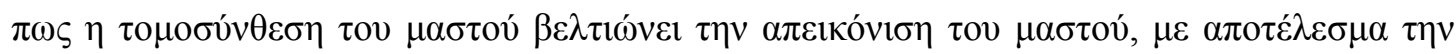

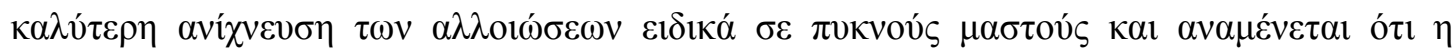

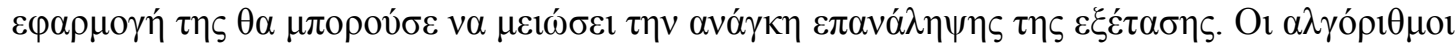

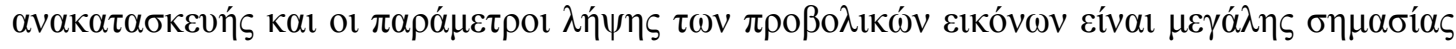

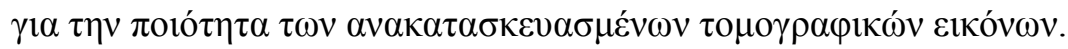

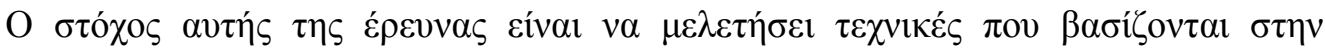

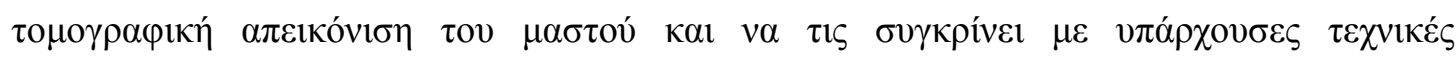

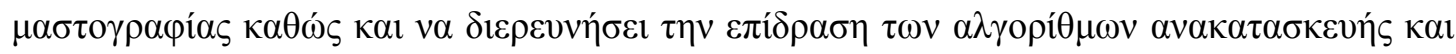

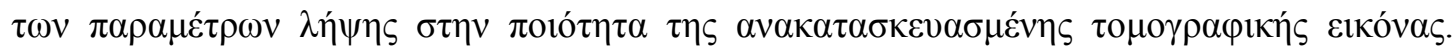

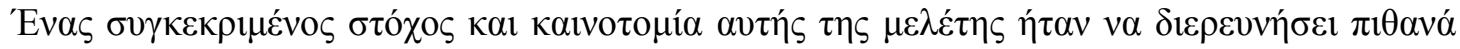

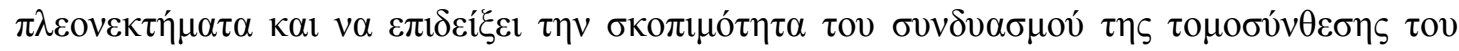

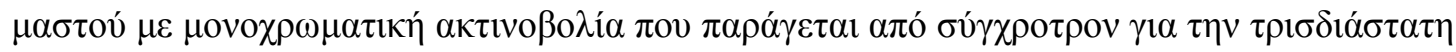

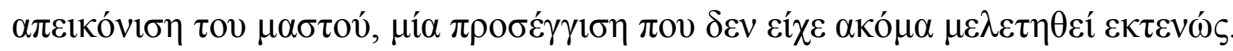

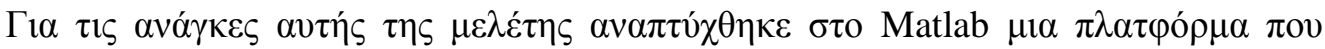

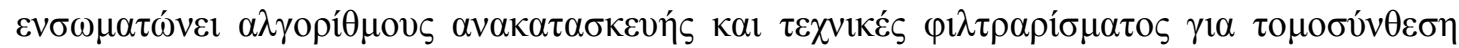

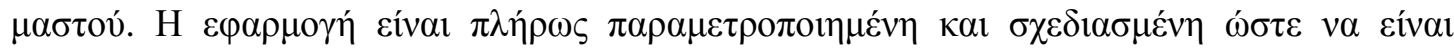

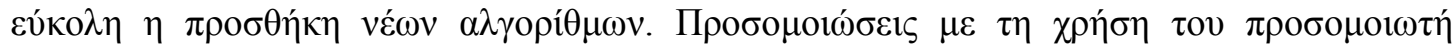

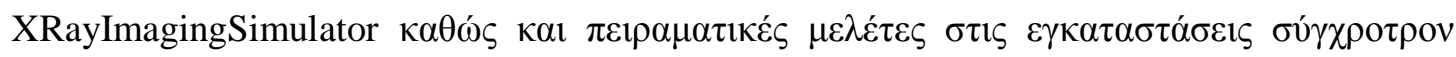

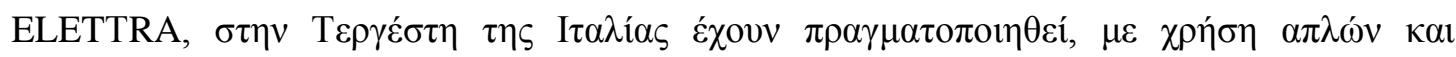

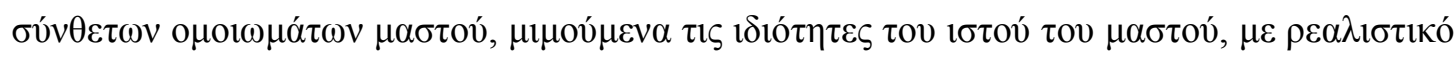

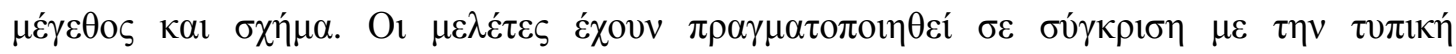

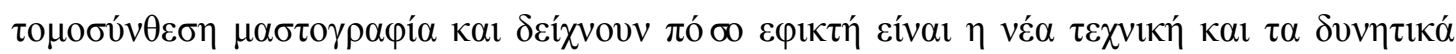

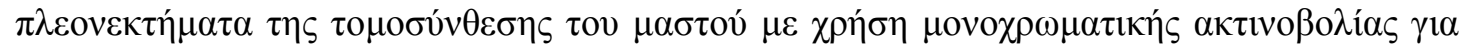

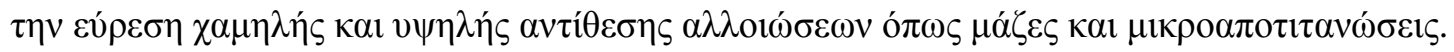




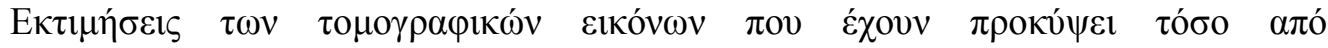

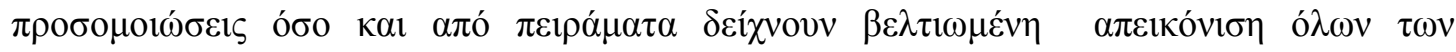

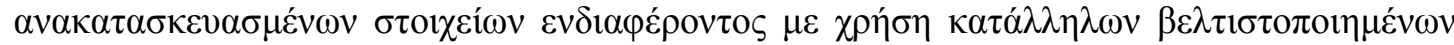

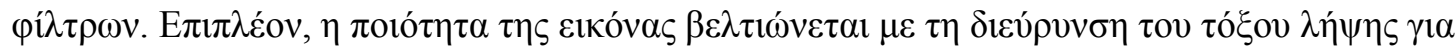

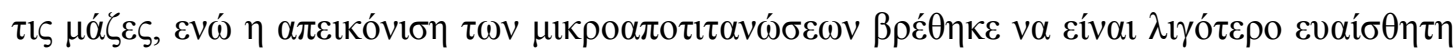

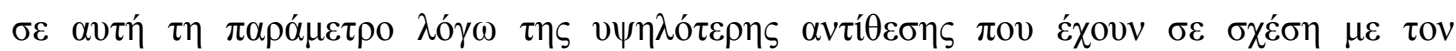

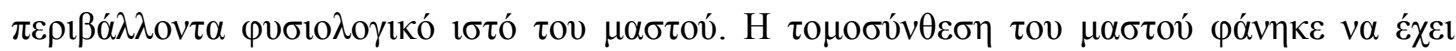

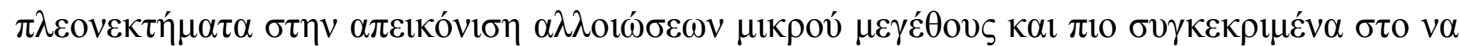

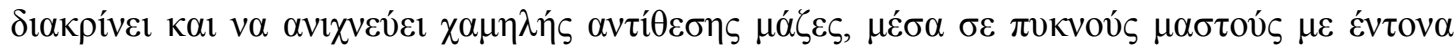

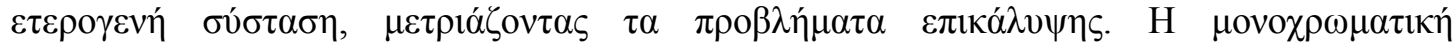

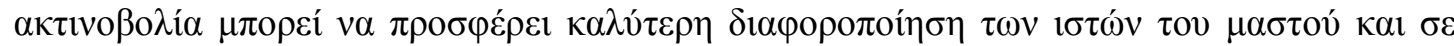

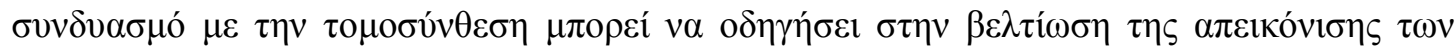

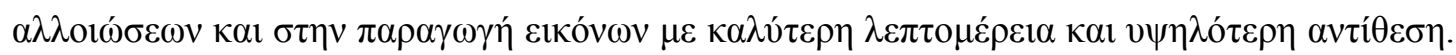

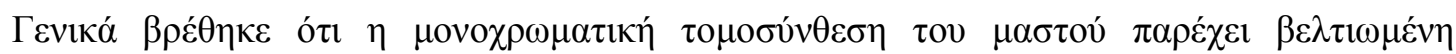

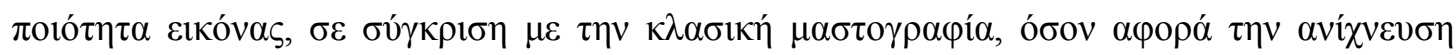

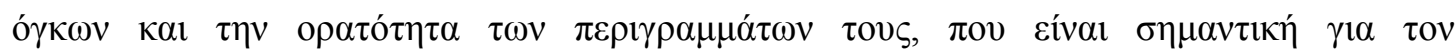

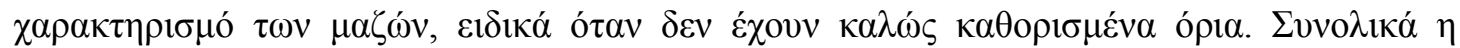

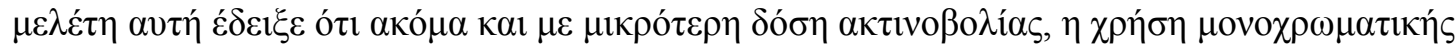

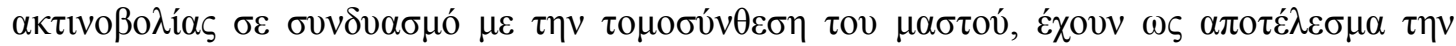

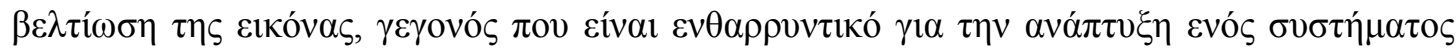

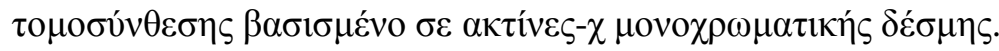




\section{Publications}

\section{Publications in international peer-reviewed journals:}

Malliori A, Bliznakova K, Speller R, Horrocks J, Rigon L, Tromba G, Pallikarakis N, "Image Quality Evaluation of Breast Tomosynthesis With Synchrotron Radiation”, Med. Phys. 2012, 39(9): 5621-5634.

Malliori A, Bliznakova K, Sechopoulos I, Kamarianakis Z, Fei B Pallikarakis N,"Breast tomosynthesis with monochromatic beams: A feasibility study using Monte Carlo simulation"(submitted in Phys Med Bio, Feb 2014 and accepted for publication, April 2014)

Malliori A, Bliznakova K, Bliznakov Z, Cockmartin L, Bosmans H, Pallikarakis N, "Breast tomosynthesis using the Multiple Projection Algorithm adapted for stationary detectors " (submitted in Med Phys, March 2014)

\section{Selected publications in conference proceedings:}

Malliori A, Bliznakova K, Daskalaki A, Pallikarakis N., "Graphical User Interface for Breast Tomosynthesis Reconstructions: An application using Anisotropic Diffusion Filtering”, , XIII Mediterranean Conference on Medical and Biological Engineering and Computing MEDICON 2013, Sevilla, Spain, September 25-28, 2013, IFMBE Proceedings 2014, Vol. 41, pp. 479-482.

Malliori A, Bliznakova K, Dermitzakis A, Pallikarakis N., "Evaluation of the Effect of Acquisition Parameters on Image Quality in Digital Breast Tomosynthesis: Simulation Studies”, World Congress on Medical Physics and Biomedical Engineering - WC2012, Beijing, China, May 26-31, 2012, IFMBE Proceedings 2013, Vol. 39, p. 2211-2214.

Malliori A, Bliznakova K, Pallikarakis N., "Digital Breast Tomosynthesis With Monochromatic Beam for Low Contrast Features”, Riga Technical University $53^{\text {rd }}$ International Scientific Conference. International Symposium on Biomedical Engineering and Medical Physics, Riga, Latvia, October 10-12, 2012.

Malliori A, Bliznakova K, Pallikarakis N., "Dual Energy Studies with Synchrotron Radiation: Preliminary Results for Low-Contrast Features”, European Medical Physics and Bioengineering Conference - EMPEC 2012, Sofia, Bulgaria, October 18-20, 2012.

Malliori A, Bliznakova K, Pallikarakis N., "A Computer-Based Platform for Digital Breast Tomosynthesis Simulation Studies", International Conference on Information Technology Applications in Biomedicine - ITAB, Corfu, Greece, November 2-5, 2010, Proceedings of the IEEE/EMBS Region 8. 
CHAPTER 1

INTRODUCTION

\section{CHAPTER SUMMARY}

This chapter is an introduction to the studied problems. It outlines the motivation and describes analytically the objectives of the present thesis. A thesis outline is also included, summarizing the scope and content of each one of the following chapters. 


\subsection{MOTIVATION}

Developments in the field of Breast Tomosynthesis (BT) during the last decade resulted in this $\mathrm{x}$-ray tomographic technique being presently introduced in routine everyday clinical practice. This method allows pseudo three-dimensional (3D) representation and could contribute in screening and diagnosis of breast cancer in its nascent stages. The potential of this new technique to detect malignant or benign breast masses, particularly for very dense breasts, has been summarized in the review article of Baker and Lo (Baker and Lo 2011) and has been demonstrated in several clinical trials (Haas et al 2013, Skaane et al 2013, Ciatto et al 2013, Rose et al 2013). The complete replacement of the x-ray mammography, that is currently the 'gold' standard technique used for screening and diagnosing breast masses, with BT is however associated with solving important questions such as single or double view tomosynthesis, training of radiologists, cost, etc. Nowadays, research is performed towards optimizing the image acquisition process as well as the post-acquisition aspects, including reconstruction, image processing, and analysis (Sechopoulos 2013a, Sechopoulos 2013b). Optimization of image acquisition is towards optimization of scanning geometry and related parameters (distances, arc length, number of projections), beam (tube voltage, total exposure), detector parameters and dose. Optimization of reconstruction algorithms including prefiltering and post-processing techniques in respect to the type of feature characteristics is also an important step that can influence the detection of breast cancer.

\subsection{STUDIED PROBLEMS AND OBJECTIVES}

Presently, BT studies are performed with polychromatic beams. Synchrotron radiation, owns characteristics such as high brightness and intensity, narrow angular collimation and the ability to produce photons of certain desirable energies (monoenergetic), which may open a new era in breast imaging. There is ongoing research on the use of monochromatic x-ray sources, and investigations in the field of mammography have shown that the use of monochromatic beams in the range $16 \mathrm{keV}$ to $24 \mathrm{keV}$ may provide increased image quality compared to conventional mammography at equivalent dose (Burattini et al 1994, Burattini et al 1995, Johnston et al 1996, Arfelli et al 1995, Moeckli et al 2000, Diekmann et al 2004, Baldelli et al 2005, Dreossi et al 2007).

Our overall goal is to study and evaluate the potential of a breast tomosynthesis setup using monochromatic radiation. Throughout this research, simulations and experiments were carried out using simple homogeneous and more complex, heterogeneous breast-like phantoms. The first phase was dedicated to incorporate validated tomosynthesis reconstruction algorithms 
and filtering methods into a computer-based platform for BT. These algorithms were adapted for the geometry of a conventional clinical BT setting and evaluated using real projection images. Reconstructions based on data acquired with monochromatic beams at Synchrotron facilities were also performed. The second phase involved an optimization study for the geometry of Synchrotron, in terms of filtering methods and acquisition parameters for features of different size and contrast while the final phase includes a thorough comparison between tomosynthesis using monochromatic and polychromatic beams as well as evaluation against conventional mammography.

\subsection{THESIS OUTLINE}

Chapter 2 summarizes the anatomy and imaging of the female breast using X-rays and specifically BT tomographic technique. A review of BT is presented outlining the present considerations and future trends. The basic principles of synchrotron radiation are presented in Chapter 3, which also refers to synchrotron facilities available providing monochromatic beams. The advantages and disadvantages of using monochromatic beams compared to polychromatic in terms of dose and image quality as well as imaging procedure and system complexity are demonstrated and specifically discussed for mammography. This chapter also outlines the potential of using monochromatic beams with BT and how this technique could be adapted in a clinical setting. Chapter 4 describes the computer-based platform that was developed for dedicated BT studies. The scope of this platform was to incorporate the Multiple Projection Algorithm (MPA) and the BackProjection (BP) algorithm combined with several filters. The algorithms that are compatible with any image acquisition geometry, source and detector type, have been adapted and are presented in detail for partial isocentric rotation with stationary detector which is the most common acquisition geometry for BT. Chapter 5, presents a study evaluating these algorithms for the specific geometry of a Siemens MAMMOMAT Inspiration clinical system and real projection images were acquired with the BR3D-CIRS breast phantom for this purpose. The evaluation involved tomosynthesis images reconstructed with MPA and BP as well as a comparison with 2D mammograms. Aspects studied include: the influence of the total phantom thickness, the position of the slab containing the objects of interest within the breast phantom, and the relationship between feature size and performance of reconstruction techniques. Chapter 6 is dedicated to monochromatic BT. The performance of using BT with monochromatic irradiation has been studied thoroughly for the detection of low and high contrast breast lesions. The investigations involved simulation and experimental work for the optimization of the acquisition parameters and filtering methods using simple mathematical representation of the 
women breast, composed through combination of simple geometrical primitives and complex breast phantoms. Simulated images were formed using the XRAYImagingSimulator while the reconstructions were performed with the BT platform. Experimental validation was accomplished at ELETTRA synchrotron facilities in Trieste. The second part of the experimental work, involved acquisitions of projection images with the objective to investigate potential benefits of using monochromatic beams compared to polychromatic, in image quality and features' visibility. This initial investigation involving monochromatic and polychromatic 2D images, was extended for BT; a feasibility study was performed aiming to compare the performance of BT using monochromatic over polychromatic beams under the same acquisition conditions. The following simulation studies were carried out: (a) simulation of polyenergetic BT, (b) simulation of monochromatic BT and (c) mammography simulations with a simple (homogeneous) and a complex (heterogeneous) software breast phantom. Chapter 7, summarizes the results and the concluding remarks of the present thesis and points out future research directions in this field. 
CHAPTER 2

BREAST X-RAY IMAGING AND DIGITAL TOMOSYNTHESIS

\section{CHAPTER SUMMARY}

Chapter 2 summarizes the anatomy and imaging of the female breast using $\mathrm{x}$-rays and specifically BT tomographic technique. A review of BT is presented outlining the present considerations and future trends in relation to (a) the x-ray systems and acquisition geometries that have been proposed and used, (b) radiographic machines for BT purpose used currently in clinical practice, (c) acquisition parameters, spectra and incident exposures, (d) algorithms used to reconstruct the scanned volume, (e) filtering techniques and noise removal methods and (f) evaluation metrics. 


\subsection{BREAST ANATOMY AND CONVENTIONAL MAMMOGRAPHY}

\subsubsection{Anatomy of the normal breast}

The breast is an organ whose structure reflects its special function, namely the production of milk for lactation. Anatomically, the adult breast lies on the pectoralis major and extends usually from the second to the sixth rib. A part of the gland called the "axillary tail" extends towards the axilla along the lateral border of the pectoralis major. This is important because a mass of breast cancer can develop there, even though it might not seem to be part of the breast.

Descriptions and anatomical diagrams of the gross anatomy of the lactating human breast are based on meticulous dissections of the breasts of lactating cadavers (Fig. 2.1) by Sir Astley Cooper in 1840 (Cooper 1840), and have changed little in recent decades on the basis of modern imaging modalities.

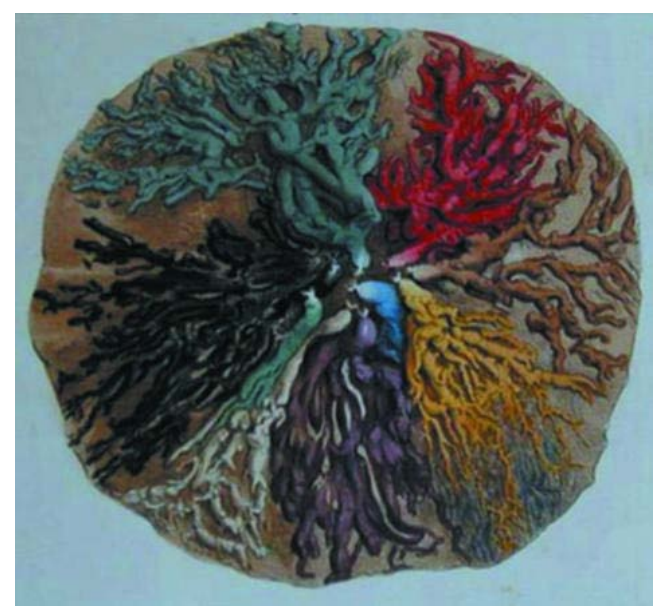

Fig. 2.1. Artist's impression of the lobes of the breast. The ducts were injected with colored wax prior to dissection of the breast of lactating cadavers (reproduced from Cooper 1840).

The breast, as described in Grey’s Anatomy (Bannister et al 1995), is composed of glandular (secretory) and adipose (fatty) tissue in a wide variation of distribution between individuals, supported by a loose framework of fibers, called Cooper's ligaments. This variety in breast glandularities, depends on parameters such as the age and is not observed between breasts of the same woman.

The glandular tissue, consists of several lobes (15 to 20) that are comprised of lobules (Fig. 2.2). The lobules further consist of clusters of alveoli containing lactocytes (mammary secretory epithelial cells) that synthesize breast milk (Tobon and Salazar 1975). The alveoli are connected to small ducts that merge into larger ducts and finally end to one lactiferous 
duct. Thus, its own lactiferous duct having a tree-like structure drains each lobe. Then, each single lactiferous duct widens into a lactiferous sinus and narrows again terminating at the nipple. Figure 2.2, depicts also the cooper ligaments and the adipose tissue which is situated between the lobes and in the space between the lobes and the skin (subcutaneous fat).

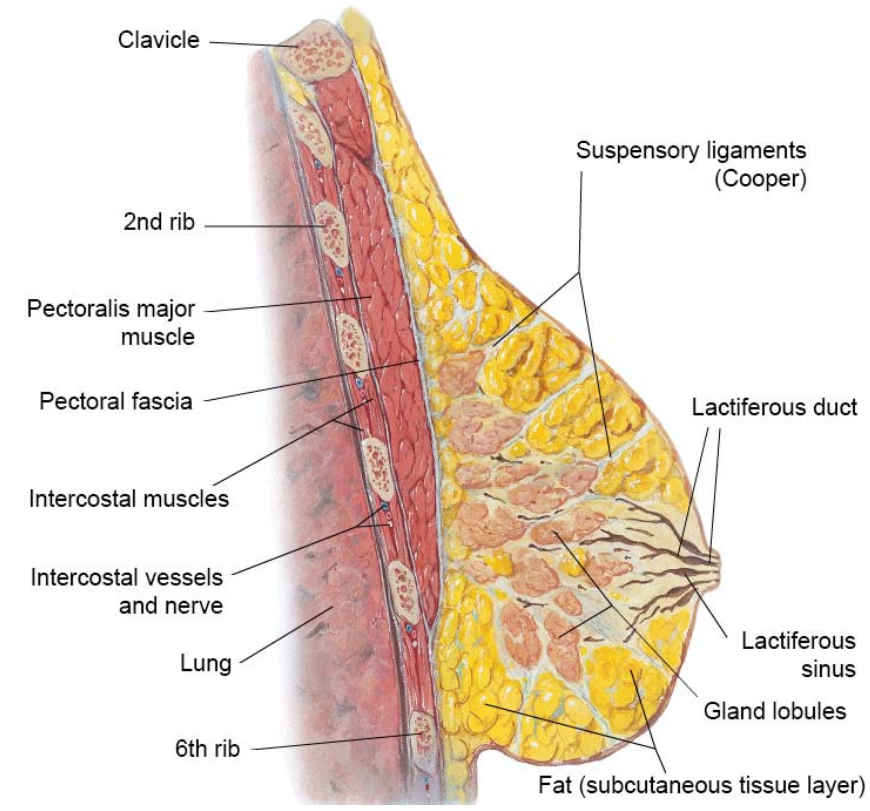

Fig. 2.2. Sagittal section of mammary gland (image adapted from Netter F.H Atlas of Human Anatomy $4^{\text {th }}$ Ed. Saunders, Netter 2006).

The blood supply from the breast comes primarily from the internal mammary artery (60\%), which runs underneath the main breast tissue and the lateral mammary branch of the lateral thoracic artery (30\%) while in smaller parts, from the posterior intercostal arteries and the pectoral branch of the thoracoacromial artery (Cunningham 1977, Bannister et al 1995).

The lymph is drained from the breast by two main pathways. The majority of lymphatic vessels flow to the axillary nodes, while the rest flow to internal mammary lymph nodes located deep to the breast. Knowledge of this lymphatic drainage is essential because in case of a breast cancer metastasis, the first lymph node of the chain is usually affected.

\subsubsection{Mammography}

Mammography is the process of imaging the female breast using low-energy x-rays (usually around $30 \mathrm{kVp}$ aiming to detect and diagnose breast cancer at an early stage. This can be achieved only with specially designed radiographic equipment dedicated to mammography. 
During the procedure, the breast is kept still using compression plates, which apart from preventing motion blur, is aiming to reduce the thickness of breast tissue that x-rays must penetrate and therefore the amount of scattered radiation. The mammographic image depicts the result of the interactions between the breast tissue and the $\mathrm{x}$-ray photons. The $\mathrm{x}$-ray photons coming out of the breast, reach the detector after passing through an anti-scatter grid (Fig 2.3) which can reduce scatter-to-primary ratio for a large or dense breast by as much as a factor of 3 . When fine detail must be examined, an air gap not only leads to magnification and (when used with a microfocus tube) improved resolution, but also reduces scatter. Either a grid or an air gap increases the average dose to the breast, however the benefits of increased clarity far outweigh any increment in radiation risk, especially for difficult cases.

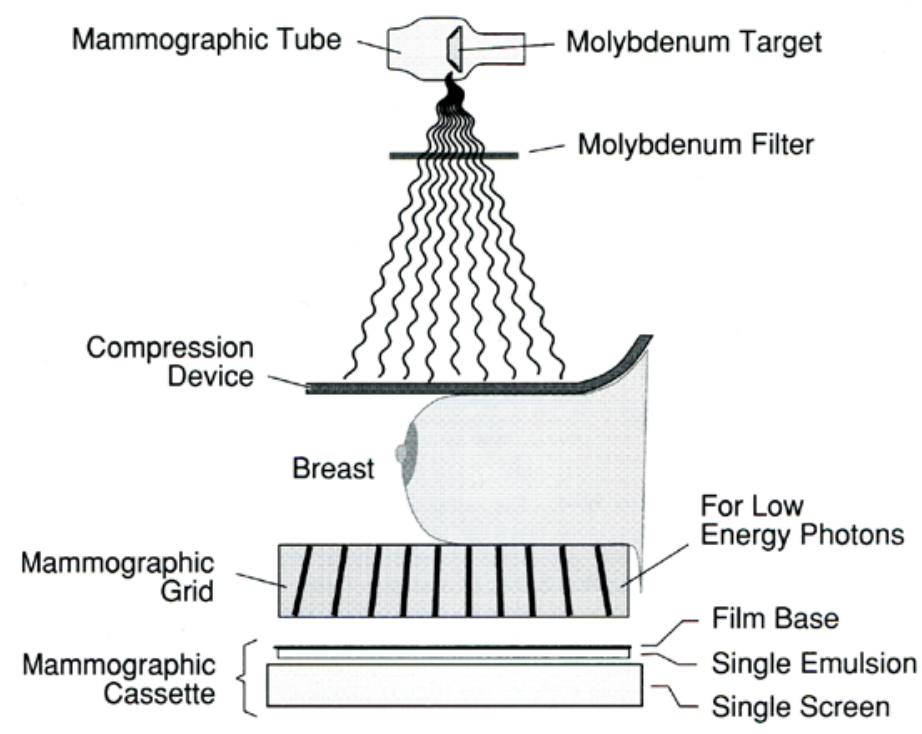

Fig. 2.3. A dedicated mammographic system employing x-ray tube with molybdenum target and a molybdenum filter; a compression device; a low-photon energy grid; and a single-screen detail cassette containing single-emulsion film.

The intensifying screens used for mammography are designed to produce very low blurring and good visibility of small structures and objects. In order to detect small changes (in attenuation and size) in breast tissue, it is necessary to have a film-screen combination which can detect very small differences at a low dose. Periodic mammographic screening has been found to result in the earlier detection of breast cancers, leading to a reduction in patient mortality and morbidity (Paci 2012, Paap et al 2010).

Early breast cancer is difficult to detect because it appears similar to normal breast tissue. The use of low-energy $\mathrm{x}$-rays enhances the subject contrast as the attenuation coefficients of the soft tissues and the differences among them (which is the important thing) are greatest 
when imaging with low-energy photons The photoelectric effect dominates and the linear attenuation coefficients of normal and pathologic tissues differ by a factor of:

$$
\frac{\mu_{\text {norm }}}{\mu_{\text {path }}} \sim \frac{\rho_{\text {norm }}}{\rho_{\text {path }}}\left(\frac{Z_{\text {norm }}}{Z_{\text {path }}}\right)^{3}
$$

where $Z_{\text {norm }}, Z_{\text {path }}$ and $\rho_{\text {norm, }}, \rho_{\text {path }}$ are the effective atomic numbers and densities, respectively.

Likewise, although contrast is rarely an issue in imaging bones, for microcalcifications, enhanced visibility brought about by low $\mathrm{kVp}$, can increase the likelihood of their detection (Wolbarst 1993).

In all x-ray imaging methods there is always a balance between image information and the absorbed dose to the patient. In mammography, especially for detection of cancer at an early stage when it can be treated most effectively, high contrast sensitivity, high detail, and low noise are necessary. Each of these characteristics as well as the x-ray beams with low penetrating characteristics needed to produce visible images, require a relatively high $\mathrm{x}$-ray exposure.

The dose to the breast of an individual patient is determined by a combination of three factors: (i) the characteristics of the equipment being used, (ii) the technique factors selected for the examination, and (iii) the size and density of the patient's breasts. The radiation beam of each mammography machine is usually calibrated so that it delivers a specific and known absorbed dose to a reference (average size) breast when imaged with a specific set of technique factors. The major factors determining this dose are the sensitivity of the receptor (film-screen combination, characteristics of digital receptors), and the setting of the automatic exposure control (AEC) level to produce a specific film density. With regard to the technique, the selected $\mathrm{kV}$ (typical range of $24 \mathrm{kV}$ to $32 \mathrm{kV}$ ) and anode/filter combination are the major factors that determine the dose. In case the $\mathrm{kV}$ is too high then the contrast and, therefore the visibility, will be reduced. Lower $\mathrm{kV}$ values are used to enhance contrast but do not provide sufficient penetration through thick or dense breast tissue where higher $\mathrm{kV}$ values are required. When the $\mathrm{kV}$ is reduced in a specific examination, the mAs must be raised to produce an acceptable overall optical density. The combination of reduced $\mathrm{kV}$ and increased $\mathrm{mAs}$ results in a higher dose. Dose generally increases with increased breast size and density, for a given optical density, because more radiation is required to penetrate the breast and expose the receptor.

The quantity that is used to measure the dose to the breast is the mean glandular dose (MGD). This is based on the assumption that it is the glandular tissue in the breast, and not the adipose 
tissue or fat that is most sensitive to radiation effects. The MGD is defined as the mean (average) dose to the glandular tissue. It is considered to be a reasonable quantity for comparing relative risk from different mammography procedures.

To ensure the necessary image quality with the lowest possible dose, mammography should be performed by highly qualified radiographers with the ability to evaluate each patient's breast and then select the optimum $\mathrm{kV}$ and other exposure parameters.

The radiographic appearance of the breast varies depending on the relative distribution of fat and glandular tissue, with the first being radiologically lucent and appear dark while the latter being characterized as radiologically dense and appear brighter, in the mammographic image. These variations are associated with breast cancer risk (Boyd et al 1998, Li et al 2005, Boyd et al 2010) and some examples of different types of mammograms are illustrated in Fig. 2.4.
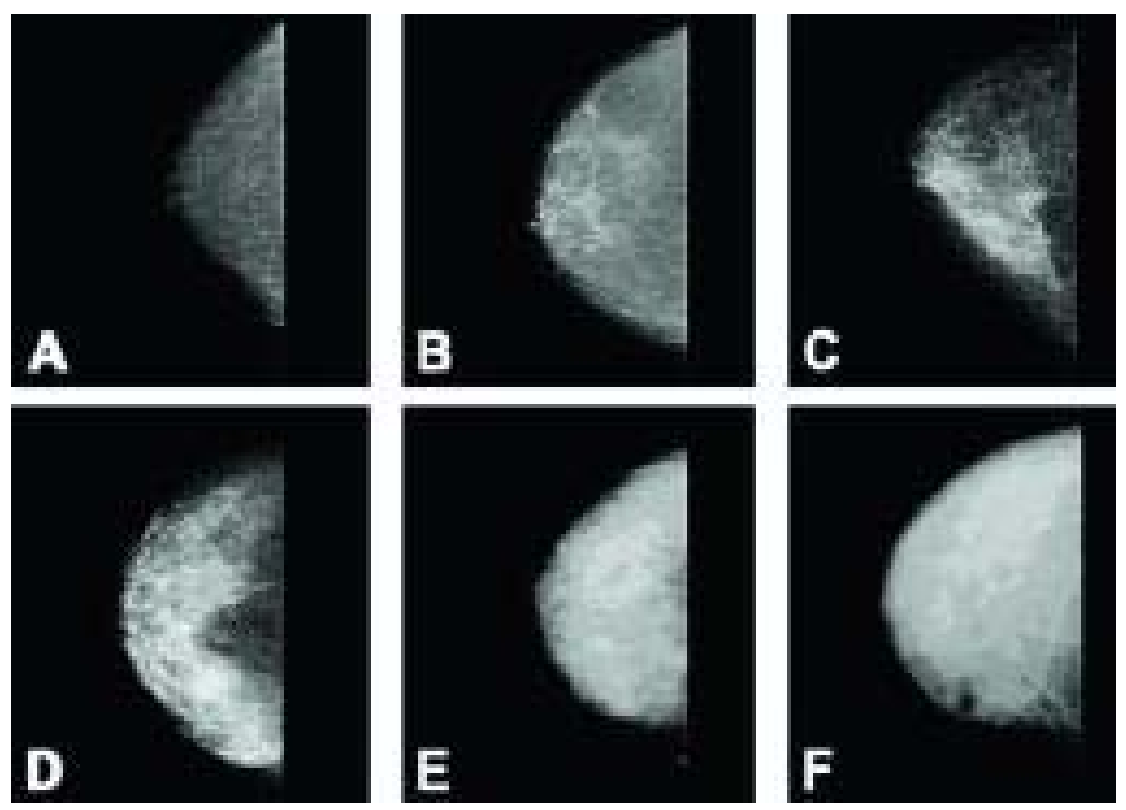

Fig. 2.4. Examples of variation in mammographic density: (A) $0 \%$, (B) $<10 \%$, (C) $<25 \%$, (D) $<50 \%$ (E) $<75 \%$ and (F) $>75 \%$ (image adapted from (Boyd et al 2010).

Digital imaging is replacing conventional imaging in general and also in mammography. Information is quickly available on the screen for making a diagnosis, it can be transmitted electronically to others and images can be printed on films as necessary. Screen-film mammography (SFM) was the standard technique in breast cancer screening for many years, but today the most common imaging procedure is a two-view (medio-lateral oblique and cranio-caudal) examination using full-field digital mammography (FFDM). 
Full-field digital mammography has several potential benefits as compared with SFM in mammography screening. Digital technology also opens for implementation of advanced applications, including computer-aided detection (CAD) (Bassett 2000, Chan et al 2005, Costaridou et al 2008, Elter and Horsch 2009, Karahaliou et al 2012). Phantom studies and experimental clinical studies have shown that FFDM is equal or slightly superior to SFM for detection and characterization of mammographic abnormalities (Skaane 2009).

\subsubsection{Breast abnormalities}

Mammographic findings should be described, evaluated and classified in a harmonized way in order to standardize reporting, reduce confusion in breast imaging interpretations, and facilitate outcome monitoring. For that purpose the Breast Imaging Reporting and Data System (BI-RADS) has been developed as the result of a collaborative effort between members of various committees mainly of the American College of Radiology (ACR).

The assessment following mammography (Table 2.1) can range from 0 (Incomplete) to 6 (Known biopsy - proven malignancy) according to the BIRAD category resulting in the corresponding recommendations for further clinical management.

Table 2.1. BIRADS classification for management of abnormal mammograms

\begin{tabular}{|c|c|c|}
\hline BI-RADS Category & Assessment & $\begin{array}{c}\text { Clinical Management } \\
\text { Recommendation(s) }\end{array}$ \\
\hline 0 & Assessment incomplete & $\begin{array}{c}\text { Need to review prior studies and/or } \\
\text { complete additional imaging }\end{array}$ \\
\hline 1 & Negative & Continue routine screening \\
\hline 2 & Benign finding & Continue routine screening \\
\hline 3 & Probably benign finding & Short-term follow-up mammogram \\
\hline 4 & Suspicious abnormality & Perform biopsy \\
\hline 5 & Highly suggestive of malignancy & Biopsy and treatment, as necessary. \\
\hline 6 & $\begin{array}{c}\text { Known biopsy-proven } \\
\text { malignancy }\end{array}$ & $\begin{array}{c}\text { Assure and follow-up the respond } \\
\text { to treatment }\end{array}$ \\
\hline
\end{tabular}

X-ray imaging of benign or malignant breast pathologies results in mammographic findings in the form of masses, microcalcifications ( $\mu \mathrm{Cs}$ ), architectural distortion of normal tissue 
patterns and asymmetry between corresponding regions of images of the left and right breast (Yaffe 2003). These abnormalities are described according to the BIRAD lexicon as follows:

\section{$\underline{\text { Mass }}$}

A "Mass" is a space occupying lesion in two different projections. If a potential mass is seen in only a single projection it should be called a "density" until its three-dimensionality is confirmed.

Circumscribed (well-defined or sharply-defined) margins: The margins are sharply demarcated with an abrupt transition between the lesion and the surrounding tissue. Without additional modifiers there is nothing to suggest infiltration.

Indistinct (ill defined) margins: The poor definition of the margins raises concern that there may be infiltration by the lesion and this is not likely due to superimposed normal breast tissue.

Spiculated margins: The lesion is characterized by lines radiating from the margins of a mass.

\section{$\underline{\text { Architectural distortion }}$}

The normal architecture is distorted with no definite mass visible. This includes spiculations radiating from a point, and focal retraction or distortion of the edge of the parenchyma. Architectural distortion can also be an associated finding.

\section{Asymmetric density}

This is a density that cannot be accurately described using the other shapes. It is visible asymmetry of tissue density with similar shape of two views, but completely lacking borders and the conspicuity of a true mass. It could represent an island of normal breast, but its lack of specific benign characteristics may warrant further evaluation. Additional imaging may reveal a true mass or significant architectural distortion.

\section{Calcifications}

Amorphous or indistinct calcifications: These are often round or "flake" shaped calcifications that are sufficiently small or hazy in appearance that a more specific morphologic classification cannot be determined.

Pleomorphic or heterogeneous calcifications: These are usually more conspicuous than the amorphic forms and are neither typically benign nor typically malignant irregular calcifications with varying sizes and shapes that are usually less than $0,5 \mathrm{~mm}$ in diameter. 
Fine, linear or fine, linear, branching (casting) calcifications: These are thin, irregular calcifications that appear linear, but are discontinuous and under $0,5 \mathrm{~mm}$ in width. Their appearance suggests filling of the lumen of a duct involved irregularly by breast cancer.

Benign calcifications: Benign calcifications are usually larger than calcifications associated with malignancy. They are usually coarser, often round with smooth margins and are much more easily seen.

\subsection{DIGITAL BREAST TOMOSYNTHESIS}

\subsubsection{Principles of Tomosynthesis}

Digital Tomosynthesis (DTS) is a tomographic technique that permits acquisition of a number of projection images similar to conventional x-ray images, over a limited angular range, in order to produce slice images of the inspected volume (Dobbins and Godfrey 2003). The twodimensional projections can provide three-dimensional information of the imaged object using generally simple reconstruction algorithms. Several authors, (Miller et al 1971, Garrison et al 1969)have demonstrated tomosynthesis with film as a detector using methods derived by the Dutch researcher Ziedses des Plantes who in the '30s introduced tomographic imaging to the medical research community (Ziedses Des Plantes 1932). The switch from screen-film to electronic means of image acquisition, simplified significantly the tomosynthesis process which however could not compete with Computed Tomography (CT) that became widely accepted by the late '70s. The interest in DTS was regained in the late '80s with the development of algorithms that can be applied in tomosynthesis and enabled the reconstruction of arbitrary planes of any direction relatively to the fulcrum plane. Kolitsi et al. described such an algorithm based on translating group of pixels rather than on an individual basis called the Multiple Projection Algorithm (MPA), resulting in a significant reduction of computing times (Kolitsi 1991). These achievements, along with the appearance of digital flat-panel detectors in the '90s resulting in high quality images at rapid readout rates (Dobbins and Godfrey 2003), led to extensive research activity during the last years in this area.

Tomosynthesis can prove to be very useful for imaging the breast in an attempt to optimize the detection and depth localization of abnormalities with dose levels comparable to those of conventional mammography (Niklason et al 1997). Recent studies have shown, that BT can be beneficial in the characterization of lesions particularly in dense breasts (Gong et al 2006) and has the potential to reduce the recall rate (Gur et al 2009).

In order to reconstruct tomographic planes of an imaged volume, a limited number of 
projection images of this volume over an arc, is necessary to be acquired. For the acquisition, there needs to be a relative movement between the source and the detector. In most studies a full-field, flat, digital detector, which can either rotate or remain stationary is used. This type of detector is ideally suited due to its low noise, large, flat surface area with minimal image distortion and rapid image readout. The X-ray source usually moves in an arc around the imaged objector can move parallel to the detector in an opposite trajectory; however other geometries are also under investigation.

Many of the traditional values used in conventional mammography can be used in BT as well, as the two methods have most of the acquisition parameters in common. For the two parameters applicable only in tomosynthesis, the angular range covered during acquisition and the number of projections acquired, there has been a wide variety of values used in the preclinical and clinical studies reported on BT. In most studies, the angular range varies from $30^{\circ}-60^{\circ}$ whereas the number of projection is usually between 11-25 (Sechopoulos and Ghetti 2009, Suryanarayanan et al 2000, Wu et al 2003, Poplack et al 2007). These results along with optimization studies concerning these parameters performed for BT with monochromatic beams using simulations (Malliori et al 2013) and experiments (Malliori et al 2012) are presented in detail in Chapter 6. The optimum combination of parameters should be the one maximizing the angular range with the number of projections remaining close to the value determined by the necessary reconstruction quality under limited total dose (Sechopoulos and Ghetti 2009).

In general, doses in a single-view BT exam are about one to two times that of a single-view full-field digital mammography (FFDM) (Dobbins 2009). Specifically, the mean glandular doses (MGD) for BT acquisition are reported to vary between 0.6 to $4.0 \mathrm{mGy}$ (Feng and Sechopoulos 2012, Poplack et al 2007, Dobbins 2009). In the most recent among the dosimetric BT studies, Feng and Sechopoulos (Feng and Sechopoulos 2012) reported similar MGD values for an average breast ( $5 \mathrm{~cm}$ thickness, $50 \%$ glandularity), in case of BT and FFDM, being only $8 \%$ higher for the former (1.30mGy and $1.20 \mathrm{mGy}$, respectively). In this case, a combination of the two modalities (2D/3D fusion imaging) would result in a total dose within the Mammography Quality Standards Act limits for two-view screening mammography study (2.5mGy). On the other hand, for a breast of $6 \mathrm{~cm}$ thickness and $14 \%$ glandularity, BT required almost double dose compared to FFDM acquisition (2.12mGy and 1.16mGy, respectively). The results reported were for a homogeneous phantom imaged using the automatic exposure control (AEC) of the Selenia Dimension system (Hologic, Bedford, Mass). This system is one of the two BT commercial systems that are currently in use and its characteristics are presented in Table 2.2. The X-ray spectrum is an important aspect in dose 
optimization. The spectra reported in BT have been typical of those used in conventional digital mammography, but the optimum spectrum may not be finally decided upon until it is determined whether BT will be used for both masses and calcifications or just for masses (Dobbins 2009). Typically, $\mathrm{W}$ target is used along with $\mathrm{Al}$, Ag or $\mathrm{Rh}$ filters and for an average 50\% glandularity and thicknesses between 2 to $6 \mathrm{~cm}$, tube voltage varies from 26 to $33 \mathrm{kVp}$. The choice of the harder energy spectrum with target/filter combination of W/Rh, with Rh thickness of $50 \mu \mathrm{m}$ has reported higher signal-to-noise ratio over Mo spectrum and seems to be more beneficial for tomosynthesis (Zhao et al 2005).

The projection images acquired on the detector are used in order to obtain the threedimensional object. The principle is to perform appropriate movement of the source and the detector during the acquisition and suitable shifting and transformations in the projected images, in order to keep the plane of interest being projected on the same place on the detector. The result is that the plane of interest remains in focus while all the rest out-of-focus details appear blurred on the obtained tomogram. Several reconstruction techniques have been developed including the Back Projection, Multiple Projection Algorithm (Kolitsi et al 1992), iterative methods and other advanced reconstruction approaches like Algebraic Reconstruction Technique, Matrix Inversion tomosynthesis and Maximum Likelihood method (Suryanarayanan et al 2000, Wu et al 2003). Likewise the reconstruction techniques, several filtering methods have been proposed to be used along with DTS either on the projection images prior to the reconstruction or afterwards on the reconstructed tomograms. These noise removal techniques are aiming to eliminate the out-of-focus structures which may become blurred during the reconstruction process, though still exist in the image and affect image quality as they are superimposed on the tomogram.

\subsubsection{Systems and acquisition technologies}

\section{Conventional tomography}

Geometric or conventional tomography was adopted in the early tomography systems and represented a major step towards diagnostic imaging. It was implemented on a single-slice basis using a film receptor and continuous image acquisition. Specifically, linear opposing parallel path motion of the x-ray tube and film was used to acquire the projection images. Parallel path geometry (Fig. 2.5) includes any motion (e.g. linear or circular) which constraints the path of tube to lie in parallel to the plane of the receptor. 


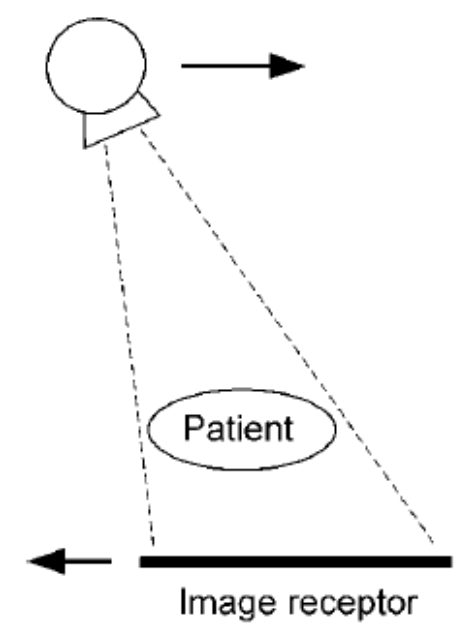

Fig. 2.5. Parallel-path tomographic geometry (figure adapted from Dobbins and Godfrey 2003).

Despite the importance of the idea, the modality had two shortcomings: The procedure allowed reconstruction only of the fulcrum plane, and thus in order to generate more than one slice, the acquisition should be repeated leading to potentially high levels of x-ray exposure to the patient; and inability to efficiently suppress out-of-plane details.

Soon afterwards, Miller et al. published their results on 'photographic laminography' (Miller et al 1971) and Grand built a prototype 3D image receptor based on circular image acquisition geometry and connected the term 'tomosynthesis' with the ability to retroactively produce an infinite number of arbitrary tomograms (Grant 1972).

\section{Tomosynthesis systems using isocentric motion}

Most early days tomosynthesis geometries of motion, involved an x-ray tube movement in a path parallel to the plane of the image receptor. However, later on certain geometries adopted in tomographic devices, including the C-arm or U-arm devices, the tube and the detector (or only the tube) move in an arc about a point called the center of rotation or isocenter. Depending on the detector's movement, this motion is further divided to complete isocentric and partial isocentric motion (Fig. 2.6). 


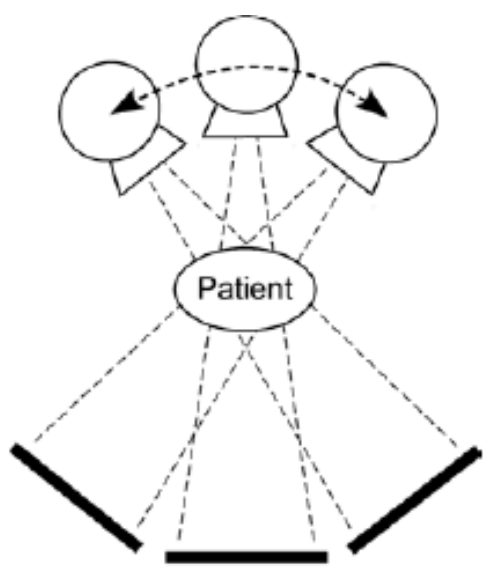

Image receptor

(a)

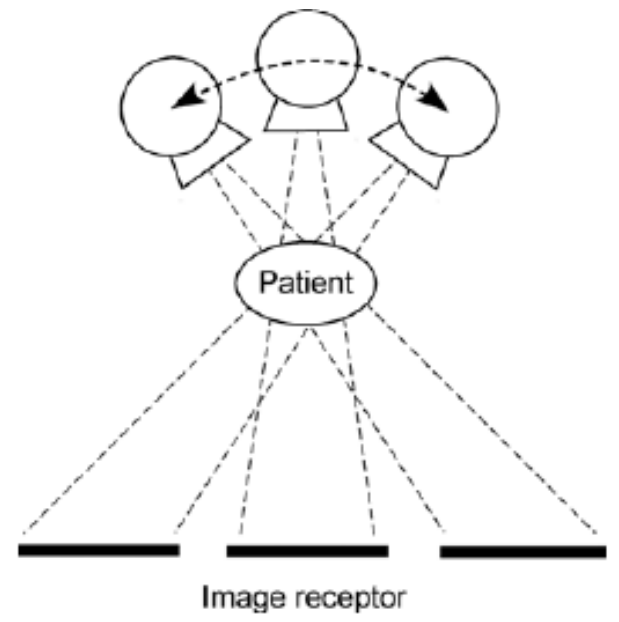

(b)

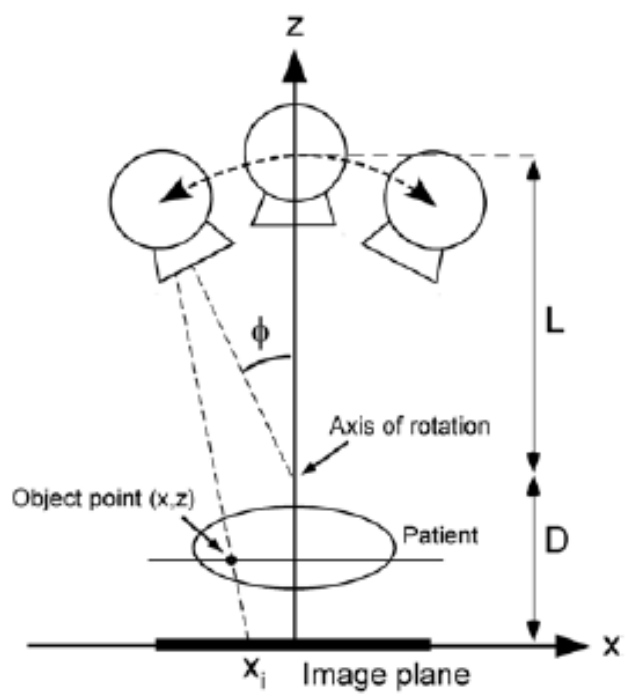

(c)

Fig. 2.6. Tomosynthesis geometries using isocentric motion. (a) Complete isocentric motion (b) Partial isocentric motion where the source moves in an arc and the detector horizontally in one plane and (c) Partial isocentric motion of (Niklason et al 1997) in which the detector is stationary (figure adapted from Dobbins and Godfrey 2003).

\section{Complete isocentric motion}

In such units [Fig. 2.6(a)], the x-ray tube and the image intensifier are locked together and rotate around a fixed point - the isocenter, while maintaining a constant although typically different distance between source to isocenter and isocenter to detector resulting in a constant magnification factor. Since the detector is rotating, its plane cannot be considered as parallel to any of the planes of interest inside the patient. This means that suitable transforms have to be applied to relate the projected image data to a plane parallel to a given plane of interest. 


\section{Partial isocentric motion}

In this type of isocentric motion, the x-ray tube moves in an arc about the center of rotation but the detector can either move in a plane [Fig. 2.6(b)] or remain stationary [Fig. 2.6(c)]. In this motion, the $\mathrm{x}$-ray tube does not travel in a line parallel to the detector, so the magnification of the structures varies with the acquisition angle in each projection, contrary to the parallel-path geometry where it remained constant.

The partial isocentric motion with stationary detector is the most typical tomosynthesis geometry for mammographic applications due to fact that the mechanical construction of the current devices used in conventional mammography can easily incorporate this type of motion.

\section{Clinical BT systems}

Currently, most of the BT systems have the same basic components as the digital mammography in terms of type of detector, breast support, compression plates and x-ray tube mounted on an arm, making these systems suitable for working in both modalities with small adjustments. The most important adjustments include the ability of the x-ray tube to rotate around a specific point between the source and the detector; adaption of different x-ray spectrum filtration suitable and optimized for BT; detector with relatively fast read-out. The characteristics and specifications of the BT systems in use or prototypes under development, including those diverging from the typical design described above, are summarized in a recent review by Sechopoulos (Sechopoulos 2013a). In Table 2.2, the specifications of the two BT commercially available systems that are currently in use, are presented. 
Table 2.2. Characteristics of BT systems in clinical use

\begin{tabular}{|c|c|c|}
\hline & $\begin{array}{ll}\text { Siemens } & \text { MAMMOMAT } \\
\text { Inspiration }^{\mathrm{a}} & \end{array}$ & Hologic Selenia Dimensions ${ }^{\mathrm{b}}$ \\
\hline Acquisition arc & $50^{\circ}$ & $15^{\circ}$ \\
\hline Number of projections & 25 & 15 \\
\hline X-ray tube & $\begin{array}{l}\mathrm{W}, 0.05 \mathrm{~mm} \mathrm{Rh} \\
\text { continuous motion }\end{array}$ & $\begin{array}{l}\mathrm{W}, 0.7 \mathrm{~mm} \mathrm{Al} \\
\text { continuous motion }\end{array}$ \\
\hline Detector & $\begin{array}{l}\text { Full field direct (a-Se) } \\
\text { size }(\mathrm{cm}): 24 \text { x30 } \\
\text { pixel size }(\mu \mathrm{m}): 85 \\
\text { stationary }\end{array}$ & $\begin{array}{l}\text { Full field direct (a-Se) } \\
\text { size }(\mathrm{cm}): 24 \times 29 \\
\text { pixel size }(\mu \mathrm{m}): 70 \quad \text { (bined } \\
2 \times 2)^{\mathrm{c}} \\
\text { rotating }\end{array}$ \\
\hline $\begin{array}{l}\text { Source to detector } \\
\text { distance }(\mathrm{cm})\end{array}$ & 65.6 & 70 \\
\hline $\begin{array}{l}\text { Detector to center of } \\
\text { rotation }(\mathrm{cm})\end{array}$ & 4.7 & 0 \\
\hline Air gap (cm) & 1.7 & 2.5 \\
\hline Reconstruction method & FBP & FBP \\
\hline
\end{tabular}

Many other modalities like Flashing tomosynthesis (Nadjmi et al 1980, Becher et al 1985, Haaker et al 1985a, Stiel et al 1993) using an array of many x-ray point sources and Tuned Aperture Tomography (Webber et al 1997) have also been proposed.

\subsubsection{Reconstruction algorithms}

\section{$\underline{\text { Shift and Add algorithm (SAA) }}$}

Shift and add algorithm reconstructs planes parallel to the fulcrum plane (the plane of pivot about which the source and detector move) (Maravilla et al 1983, Haaker et al 1985b, Rimkus et al 1989). The reconstruction approach is based on the fact that during the relative sourcedetector movement, structures from planes with different heights $\mathrm{z}$, are projected onto the detector on positions that depend on this height. Therefore, planes at different heights above or below the fulcrum plane, can be easily reconstructed by shifting the acquired projection images prior to adding, so that structures from this specific plane are lined up in focus and 
appear sharp in the final tomogram. Structures from all other planes above or below, appear on different positions in the shifted images, and thus become blurred after adding. Adjusting the shifting, enables the reconstruction of all planes parallel to the fulcrum plane each time, from one acquisition set. For the specific case of fulcrum plane, the structures of this plane are automatically in focus, so the projections are simply summed without any shifting before, as in conventional tomography.

\section{Back Projection algorithm (BP)}

The backprojection algorithm is an algorithm that can be applied in tomosynthesis and enables the reconstruction of arbitrary planes of any direction relatively to the fulcrum plane (Kampp 1986). Each pixel in the projection images is transferred backwards and deposited at the intersection of the $\mathrm{x}$-ray source with the plane to be reconstructed. The reconstruction plane can be defined in terms of rotating a reference plane about any of the three axis in any desired direction. The gray levels in the final matrix are calculated using a chosen interpolation technique and then the values are summed and divided by the corresponding summed interpolation weighting factors obtained for each pixel. This algorithm can reconstruct accurately planes of any desired direction. However, since it is performed pixelby-pixel results in long reconstruction times.

\section{Multiple Projection algorithm (MPA)}

The Multiple Projection algorithm is a DTS reconstruction algorithm of maximized efficiency capable of producing images of arbitrary orientations that has been developed at the University of Patras under the PhD thesis of Zoi Kolitsi (Kolitsi 1991). Pixels in the projection data that are subjected to operations in similar manner, are translated in groups rather than on an individual basis, resulting in a multifold reduction of required reconstruction time. For each acquired angle, the projection data is first geometrically projected onto the "horizontal plane" - a plane parallel to the reconstruction plane, passing through the intersection of the beam's central axis with the detector plane. Then this projection image is shifted by an amount depending on the position of reconstructed plane, and normalized to the magnification of the plane of the isocenter. All these images are added up, to produce a size modified image of the plane of interest. This image is formed on a plane parallel to the reconstruction plane, called "image formation plane”. MPA can reproduce planes tilted at any desired angle with respect to $\mathrm{x}$-axis with an important reduction in the reconstruction time. However, tilt angles with respect to y-axis are limited to less than $45^{\circ}$ (Kolitsi et al 1992). The analytical equations and implementation of this algorithm along with the suitable modifications performed for the geometry of partial isocentric rotation with a stationary detector can be found in Chapter 4 while Chapter 5 presents an evaluation study using MPA 
for this specific geometry.

\section{$\underline{\text { Iterative reconstruction techniques }}$}

Iterative approaches such as algebraic reconstruction techniques (ART), simultaneous iterative reconstruction technique (SIRT), iterative least squares technique (ILST) can be used to solve the simultaneous equations that describe the relationship between the voxels of the 3D object and the voxels of the 2D projections (Colsher 1977). These techniques make an initial estimate for the imaging subject and this estimate is iteratively corrected and improved so as to approximate the real object.

\subsubsection{Noise and filters}

Noise in DTS comes from a variety of sources some of which can be eliminated (electronic noise) with proper system design and operating conditions, while some others cannot be neglected, such as the photon noise. Another type of intrinsic noise, is the X-ray quantum noise coming from the statistical variation of the $\mathrm{x}$-ray photon flux which is Poisson distributed. This noise cannot be avoided, but can be reduced with increasing photons (Adachi et al 2002, Buliev 2004). Tomograms also suffer from noise due to blurred out-of-focus anatomical structures that exist in the tomograms or from artifacts and distortion of the details' shape due to the limited angle nature of DTS and insufficient number of projections that allows for a recovery of a limited space in the Fourier domain. Paying careful attention to the choice of acquisition parameters and the use of appropriate deblurring algorithms can minimize these types of artifacts (Dobbins and Godfrey 2003).

Spatial frequency filtering has been investigated as a noise removal method. One approach involves direct application of high pass filters to the original projection images prior to the reconstruction (Edholm and Quiding 1970). Thus the images are cleared from low-frequency blurry artifacts and superimposed tissue. The literature on filtered backprojection (FBP) for tomosynthesis has examined filters of different parametric forms (Mertelmeier et al 2006, Orman et al 2006). A well-known high-pass filter is the ramp filter widely used in tomosynthesis. However, this filter is not optimal in terms of its high-frequency noise amplification, and in the case of limited view tomography, it results in excessive suppression of low-frequency content contrary to a sinc filter which can vary between a standard ramp and a filter with smooth high-frequency roll-off (Van De Sompel et al 2011). This varying response can be easily achieved by tuning a single parameter and can prove very useful in BT for detecting both high-contrast and low-contrast features such as $\mu$ Cs and masses, 
respectively (Malliori et al 2012). In addition, median filters or carefully selected band pass filters (Sone et al 1991) could reduce spike artifacts or high-frequency noise in the projections. Another method combining low pass and high pass filtering in orthogonal directions on the projection images is Ectomography (Edholm et al 1980, Knutsson et al 1980, Petersson et al 1980).This technique could offer some interesting potential in circular tomosynthesis geometry but at the time it was developed, it could not be used in practice for imaging actual objects because there was no suitable large-area digital detector. Matsuo et al. (Matsuo et al 1993) proposed convolution with an inverse filter function which is derived analytically by the point spread function of the projection and the backprojection geometry. Knowing the point spread function, the x-ray absorption can be reconstructed by applying inverse filtering and the resulting tomogram presents less superimposed artifacts.

An approach known as selective plane removal was introduced by Ghosh Roy et al. (Ghosh Roy et al 1985) to solve exactly for the artifacts that occurred due to the planes adjacent to the plane of interest. In this method, knowledge of the blurring functions is incorporated into the reconstruction algorithm and blur from adjacent planes is then removed from the plane of interest. In the same filtering category, Sone et al. (Sone et al 1995, Sone et al 1996) proposes denoising based on the estimation of blurring from two layers-in front and behind the layer of interest-and then the subtraction with an appropriate weighting factor from the in focus plane in the tomogram. Kolitsi et al. used planes of the 3D reconstructed object to reproduce the blur arising from each one of them (Kolitsi et al 1993). Selected noisy planes were then used to produce a mask, which when subtracted from the tomosynthesized image, created a filtered image enriched with plane-specific information. The reduction of backprojection blur is done by determining the depth of small, high contrast objects.This method for noise removal is a two step process that involves differentiation between noise and in-plane structures and a subsequent selective suppression of unwanted information using wavelet transformation. The noise discrimination itself is based on an a priori knowledge of the plane-dependent mechanism for defocusing of structures. The comparison of three successive tomosynthesized planes can provide a noise map, which is subsequently used to remove the blur from the middle plane, by wavelet filtering (Badea et al 1998).

Iterative techniques (Ruttimann et al 1984, Engelke et al 1992, Webber et al 2000, Suryanarayanan et al 2000) were introduced to separate blur from in-plane information. In this approach, a weighted fraction of conventionally reconstructed planes is convolved with their blurring factions so as to produce an estimate of the tomographic blur which is then subtracted from the original set of reconstructions. This process is repeated and the estimate is corrected and improved after each iteration. 
Matrix inversion tomosynthesis (Dobbins 1990, Dobbins et al 1987, Godfrey et al 2006, Godfrey et al 2013) uses linear algebra in an attempt to find an exact solution of the blur using linear algebra. This technique solves effectively for the mid and high spatial frequency components of the image. However, the low spatial frequency data are sensitive to image noise, because the matrices at these low frequencies are poorly conditioned for inversion.

\subsubsection{Evaluation metrics}

Several evaluation metrics have been suggested and used for the quantitative evaluation of the quality of reconstructed features in BT and mammography images. The calculation of these metrics, involve usually the estimation of image characteristics such as the mean value and standard deviation of a Region of Interest (ROI) defined inside each feature of interest (i.e. masses and $\mu \mathrm{Cs}$ ) and of areas from the nearby background. The most commonly used, is the Contrast to Noise ratio (CNR) (Wagner 1977, Zhang et al 2006, Bliznakova et al 2010a, Lu et al 2011) defined as:

$$
\mathrm{CNR}=\frac{\left|\bar{\mu}_{f}-\bar{\mu}_{b k}\right|}{\sigma_{b k}}
$$

where $\bar{\mu}_{f}$ is the average value of the feature, while $\bar{\mu}_{b k}$ and $\sigma_{b k}$ are the average and standard deviation of the background, respectively.

This quantity is congruent to the definition of Rose's 'signal to noise ratio' (Rose 1948) measuring the detectability of a feature in a reconstruction plane and is often reported in studies as signal difference to noise ratio (SDNR) (Wu et al 2004, Samei et al 2005, Zhou et al 2007, Mertelmeier et al 2008, Sarkar et al 2009).Variations in the calculation of CNR have been reported in studies, using in the denominator the standard deviation of both the background and the feature, (Shaheen et al 2011, Van De Sompel et al 2011) or the product of mean value and standard deviation of the background (Sechopoulos and Ghetti 2009).

Another metric that is used is the Contrast (C) (Wu et al 2003, Mckinley et al 2004, Samei et al 2005, Shaheen et al 2011) defined as:

$$
\mathrm{C}=\frac{\left|\bar{\mu}_{f}-\bar{\mu}_{b k}\right|}{\bar{\mu}_{b k}}
$$

Similarly, a version using in the denominator the sum of the mean values of both the background and the feature, could be used in case of more non-uniform backgrounds (Michelson 1927).The C, contrary to CNR, uses only the mean values for the calculations and not the standard deviations. Thus, it is indicative of the difference in contrast between two 
ROIs with no estimation of the noise present in the image. With the move from film-screen to digital mammography, CNR is a more relevant parameter for image quality compared to C (Samei et al 2005, Borg et al 2012, Van Engen et al 2006). Moreover, the selection of the background has to be taken into consideration for all evaluation metrics and the background ROI should be an area with sufficient information, close to the features under evaluation, representing as close as possible the characteristics of the 'normal' breast tissue surrounding the features of interest.

The above mentioned metrics, are used for the in-plane evaluation of features. In order to estimate the reconstruction quality in the z plane and quantify the "out-of-plane" artifacts observed in planes outside the in-focus plane, the Artifact Spread Function (ASF) can be used (Zhang et al 2006, Lu et al 2011):

$$
\operatorname{ASF}(\mathrm{z})=\frac{\operatorname{CNR}(\mathrm{z})}{\operatorname{CNR}\left(\mathrm{z}_{0}\right)}
$$

where $\mathrm{z}_{0}$ is the location of the in-focus plane and $\mathrm{z}$ is the location of the off-focus plane, respectively. Instead of CNR, the corresponding mean pixel values of the signals have been also used in literature for the calculation of ASF (Wu et al 2004, Zhou et al 2007, Sechopoulos and Ghetti 2009). A quality factor combining in one metric the in-plane image quality and the vertical resolution was proposed by Sechopoulos, defined as the CNR over the ASF (Sechopoulos and Ghetti 2009).

Finally, to allow the comparison of results derived from measurements under different dose conditions, the following figure of merit (FOM) has been used (Arfelli 2000):

$$
F O M=\frac{C N R}{\sqrt{M G D}}
$$

This FOM uses the CNR and the mean glandular dose (MGD) and is based on the fact that for a given beam quality, if the system is quantum limited, CNR is proportional to the square root of the incident exposure to the breast (Samei et al 2005), based on Poisson distribution and statistics or to the mean glandular dose (MGD) (Borg et al 2012). In some studies, the entrance surface exposure (ESE) is used instead of the MGD (Mckinley et al 2004, Samei et al 2005).

Finally, more advanced image quality metrics have been used in literature such as detectability of lesions as evaluated using observer models (Chawla et al 2009, Chawla et al 2008, Gifford et al 2008, Reiser and Nishikawa 2010) or statistical analysis based on doctors' evaluations in most clinical studies, involving estimation of sensitivity and specificity of the method under evaluation, for cancer detection. 
CHAPTER 3

SYNCHROTRON RADIATION AND APPLICATIONS IN BREAST

IMAGING

\section{CHAPTER SUMMARY}

This chapter describes the basic principles of Synchrotron radiation and refers to Synchrotron facilities available providing monochromatic beams. The advantages and disadvantages of using monochromatic beams compared to polychromatic in terms of dose and image quality as well as imaging procedure and system complexity are demonstrated and specifically discussed for mammography. Finally, the chapter outlines the potential of using monochromatic beams with BT and how this technique could be adapted in a clinical setting. 


\subsection{SYNCHROTRON RADIATION}

\subsubsection{Basic principles}

Synchrotron radiation is a highly collimated and extremely intense radiation, emitted from electrons travelling at high speed when they are forced to change direction of motion, under the effect of magnetic fields. The sources of this radiation (Koch et al 1983) are high energy circular accelerators, like storage rings, consisting of circular evacuated pipes with bending magnets along the circumference (Fig. 3.1). The electrons enter the storage ring after being accelerated in a linear accelerator and their energy reaches several $\mathrm{MeV}$. In the circular accelerator, the electrons may be further accelerated to higher energies by the radio frequency (RF) electric fields, installed at the linear sections between the bending magnets. After reaching the expected energy, the electrons are forced to follow circular paths, under the action of the bending magnets. During each turn, they lose part of their energy by emitting synchrotron radiation in a narrow cone at the direction of their motion. The energy lost is regained when passing through the RF cavities. The storage ring consisting of the bending magnets along with focusing and de-focusing magnets (quadrupoles), insertion devices (undulators) and the RF cavities are shown in Fig. 3.1.

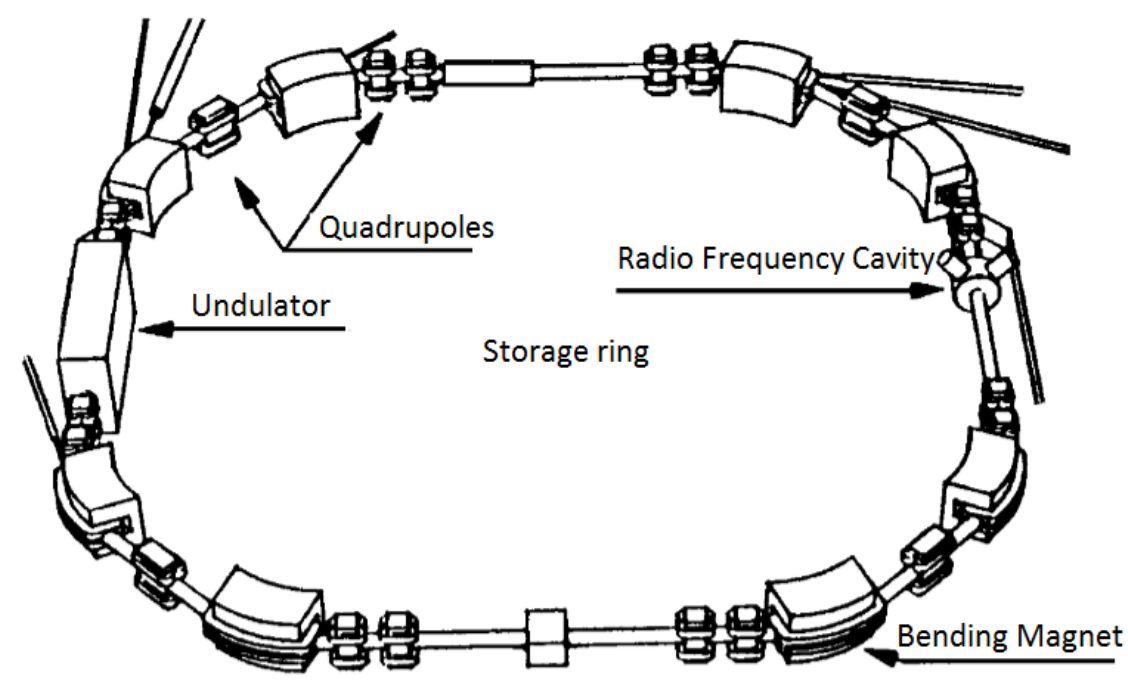

Fig. 3.1. Schematic view of a storage ring where the main elements like bending magnets (main dipoles), focusing and de-focusing magnets (quadrupoles), insertion devices (undulators) and RF cavities are visible (image adapted from (Mobilio and Balerna 2003), based on Eriksson 1988).

Synchrotron radiation can emit to a broad energy range from the infrared through the visible and ultraviolet, into the soft and hard x-ray regions of the electromagnetic spectrum, and 
owns characteristics that are extremely valuable in the study of many aspects of the structure of matter at atomic and molecular level. The basic properties of synchrotron radiation (Mobilio and Balerna 2003) depend on the characteristics of the storage ring and can be summarized to: (a) high intensity; (b) very broad and continuous spectral range; (c) narrow angular collimation; (d) high polarization; (e) pulsed time structure; (f) high brilliance of the source due to small cross section of the electron beam and high degree of collimation of the radiation; (g) ultra-high vacuum environment and high beam stability; (h) all properties quantitatively estimated by applying the methods of classical aerodynamics to the motion of relativistic charged particles.

Bending-magnet emission was the first type of synchrotron light used for practical applications, since it was automatically emitted by electron accelerators. Third generation of synchrotrons can achieve incredible brilliance using undulators (Margaritondo et al 2003). These insertion devices (Fig. 3.2), consist of a periodic structure of dipole magnets and can increase the spectral brilliance with respect to that achieved with bending magnets by several orders of magnitude. The magnetic field is alternating along the length of the undulator. Electrons traversing the periodic magnet structure are forced to undergo oscillations and thus to radiate energy. The radiation produced in an undulator is very intense and concentrated in narrow energy bands in the spectrum. It is also collimated on the orbit plane of the electrons.

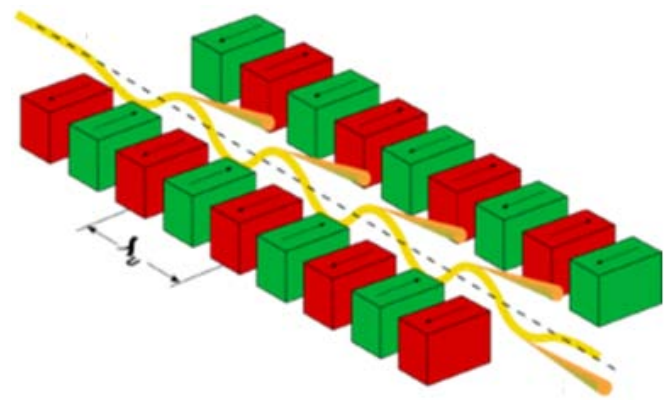

Fig. 3.2. Undulator emission (figure adapted from Holst 2005). The static field, seen by the electrons moving at relativistic speed, looks like an electromagnetic wave, whose wavelength is the period $\lambda_{u}$ of the magnet array shrunk to $\lambda_{\mathrm{u}} / \gamma$ by the relativistic Lorentz contraction. The electron oscillating under the influence of the undulator emits synchrotron light. Seen from laboratory frame, the wavelength of this emission is again Doppler-shifted, becoming $\approx \lambda_{\mathrm{u}} / 2 \gamma^{2}$ (Margaritondo et al 2003). 


\subsubsection{Synchrotron facilities}

The achievement of third generation X-ray Synchrotron radiation sources allows the production of monochromatic x-ray beams with high photon flux. The achievable brilliance, which is the main figure of merit of synchrotron radiation sources, is about $10^{12}$ times higher than a conventional x-ray tube.

The largest of those third generation synchrotron light sources, are the European Synchrotron Radiation Facility (ESRF) in Grenoble, France, the Advanced Photon Source (APS) near Chicago, USA, and Spring-8 in Japan. The list of research centers all over the world offering Synchrotron facilities, includes the Swiss Light Source located at the Paul Scherrer Institute (PSI) in Switzerland and Elettra Sincrotrone Trieste, in Italy. The latter, has more than 30 experimental stations on 27 beamlines (Fig. 3.3).

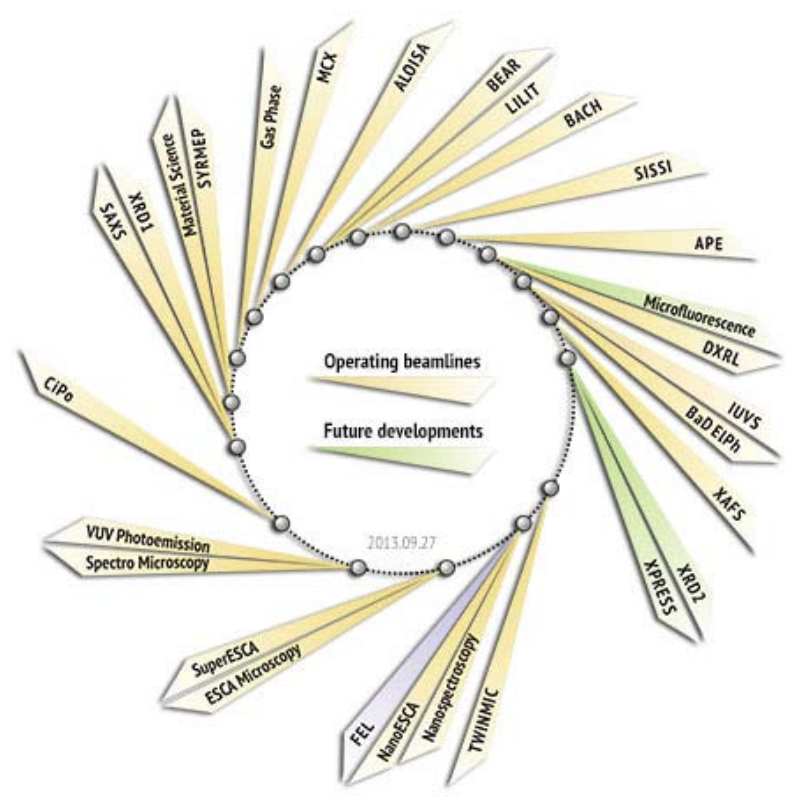

Fig. 3.3. Schematic representation of beamlines at Elettra Synchrtotron facility (image from ELLETRA webpage: http://www.elettra.trieste.it/lightsources/elettra/beamlines.html).

The SYRMEP (SYnchrotron Radiation for MEdical Physics) beamline has been designed by Sincrotrone Trieste, in cooperation with the University of Trieste and the INFN, for research in medical diagnostic radiology.

The beamline provides, at a distance of about 23m from the source, a monochromatic, laminar-section X-ray beam with a maximum area of about $160 \times 5 \mathrm{~mm}^{2}$. Figure 3.4, shows a schematic drawing of the beamline optics. The monochromator, that covers the entire angular acceptance of the beamline, is based on a double Si (111) crystals working in Bragg configuration. The crystals assembly is equipped with high precision motion stages to set the 
Bragg angle and to perform fine angular alignments of the second crystal with respect to the first. The fixed exit of the beam, independent from the selected energy, is obtained translating the second crystal with respect to the first one along a linear guide which makes an angle of about $3^{\circ}$ with respect to the beam direction. The exit beam is parallel to the incident one with a vertical displacement of $20 \mathrm{~mm}$. The useful energy range is $8-35 \mathrm{keV}$. The intrinsic energy resolution of the monochromator of $10^{-4}$ is reduced to about $4 \times 10^{-3}$ because of the natural divergence of the beam.

Prior to the monochromator, the first element after the frontend is a $2 \mathrm{~mm}$ beryllium (Be) window which divides the ultra-high vacuum of the ring from the beamline and eliminates the low frequency components of the radiation followed by a slit system which allows to select the beam size and shape with micrometric precision. A second $0.5 \mathrm{~mm}$ Be window is located after the monochromator and separates the vacuum components of the beamline from the rest of the setup. Then a second system of slits is used to shape the beam and stop the radiation scattered by the previous elements of the beamline and finally a system of two identical ionization chambers measures the intensity of the radiation while fast and safety shutters are used for the control of the exposition during the examination.

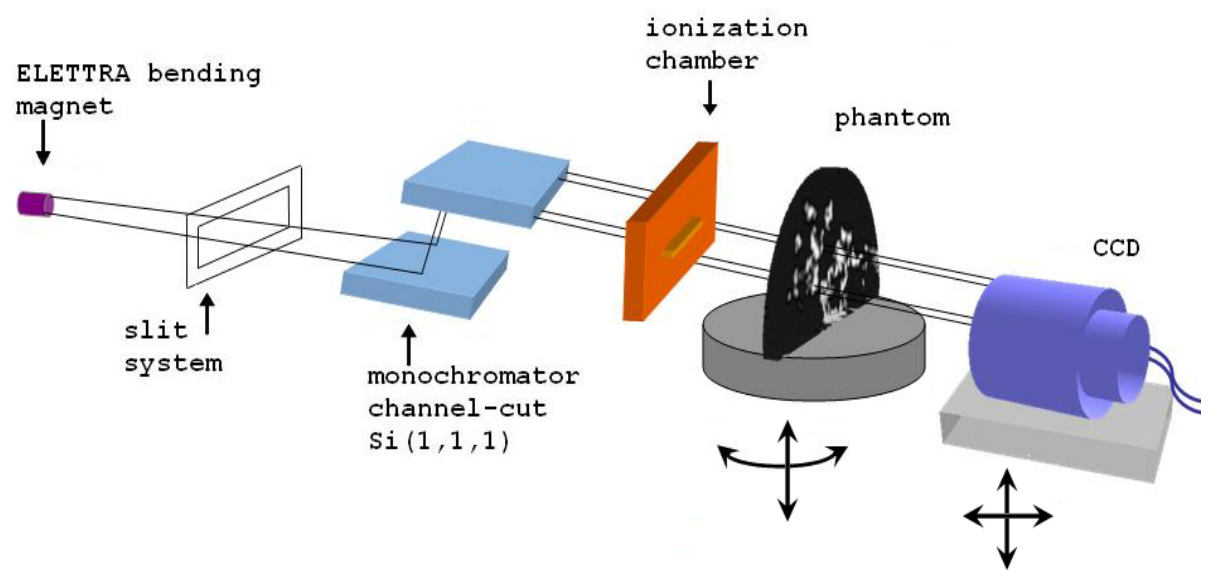

Fig. 3.4. Schematic drawing of the beamline optics (image redrawn from INFN webpage: http://w3.ts.infn.it/experiments/syrmep/ebeamline.html).

The beam then enters the experimental room. A system of motors for sample and detector movement and positioning is located on two optical tables. For the acquisition of planar images the sample is scanned through the beam, while for the tomographic imaging it is rotated in discrete steps. Typical flux measured at the sample position at $17 \mathrm{keV}$, at $2 \mathrm{GeV}$, with a stored beam of $300 \mathrm{~mA}$, is about $1.6 * 10^{8} \mathrm{ph} / \mathrm{mm}^{2}$. The available imaging techniques of 
the SYRMEP beamline are conventional absorption radiology and tomography, phase contrast imaging, diffraction enhanced imaging and the research areas include mammography, medical radiology, x-ray dosimetry, biomaterials imaging and composite materials.

The SYRMEP beamline was selected for the acquisition of data used in the experimental part of the current thesis concerning monochromatic BT and the acquisition characteristics and process are described in more details in Chapter 6.

\subsection{MONOCHROMATIC AND POLYCHROMATIC X-RAYS FOR MEDICAL IMAGING: APPLICATION FOR IMAGING THE BREAST}

Currently, in the field of medical x-ray imaging, diagnostic procedures are performed using conventional polychromatic x-ray beams. Much effort has been directed towards the choice of the anode material for the $\mathrm{x}$-ray tube, the tube voltage, the optimum spectrum for each specific imaging application including mammography (Thilander-Klang et al 1997, Dance et al 2000b, Bernhardt et al 2006) and the recently introduced to clinical practice, BT (Zhao et al 2005, Glick and Gong 2006).

The contrast in x-ray medical images is influenced by the radiation energy. For each object under investigation, an optimal energy can be found. Higher energies result usually in reduced contrast while lower energies have smaller probability to reach the detector and they are absorbed in the object increasing the delivered dose. Therefore, conventional polychromatic x-ray spectra include broad range of non-optimal energies. This has given rise to the exploring of monochromatic x-ray sources and comparison studies between monochromatic and polychromatic beams for radiographic applications.

The two basic advantages of monochromaticity, as described in McKinley (Mckinley et al 2004, Mckinley et al 2005), are:

-improved ability to separate tissues with similar attenuation coefficients since there is a maximal separation between linear attenuation values at any given $\mathrm{dE}$ along the highly nonlinear energy dependant attenuation coefficients.

- the potential of decreasing overall radiation dose to the patient while maintaining, or even improving, image quality.

Especially in the case of mammography where the differentiation between normal and cancerous tissue in $\mathrm{x}$-ray absorption imaging is very challenging it is important to optimize 
the image-quality-to-dose relationship. Although K-edge filtration and optimization of the $\mathrm{x}$ ray tube parameters and spectrum are known to reduce significantly the dose (ThilanderKlang et al 1997, Dance et al 2000b, Bernhardt et al 2006), a considerable number of low energy photons remain and filtration is limited by the resulting low fluence rate (Muller et al 2011). Investigations in the field of mammography have shown that the use of monochromatic beams in the range $16 \mathrm{keV}$ to $24 \mathrm{keV}$ may provide increased image quality compared to conventional mammography at equivalent or even lower doses (Burattini et al 1994, Burattini et al 1995, Arfelli et al 1995, Moeckli et al 2000, Baldelli et al 2005, Diekmann et al 2004, Yoon et al 2012). In a study involving both phantoms and breast specimens, Burattini et al. reported improved image quality with monoenergetic beams of $17 \mathrm{keV}$ and $18 \mathrm{keV}$ compared to conventional mammographic units, while keeping the dose at the same level (Burattini et al 1994, Burattini et al 1995). Moeckli et al. performed similar comparisons using test objects (Moeckli et al 2000) while Arfelli et al. in 1995 reported the design of a monoenergetic beam dedicated to x-ray breast imaging at Elettra Synchrotron facility (Arfelli et al 1995). The system design involves the possibility of performing both planar mammography and breast tomography (Arfelli et al 1995, Arfelli et al 1996, Arfelli et al 1998b, Pani et al 2004, Chen et al 2010).

Approaches that could exploit the advantages of monochromaticity and at the same time could be adapted in clinical systems using quasi-monochromatic beams for $\mathrm{x}$-ray mammography and mammotomography applications, have been also reported (Hoheisel et al 2005, Hoheisel et al 2006, Gambaccini et al 2001, Mckinley et al 2004, Mckinley et al 2005). Initial simulation and experimental results were promising (Mckinley et al 2004, Mckinley et al 2005). In these studies, quasi-monochromatic spectra were achieved by inserting cerium filters $\left(Z=58, \rho=6.770 \mathrm{~g} / \mathrm{cm}^{3}\right.$ of thickness $0.2,0.5$ and $0.7 \mathrm{~mm}$ (Santoku America, Inc., Tolleston, AZ) into a custom built collimator. The shutters of the collimator were as closed as possible in order to obtain scatter reduction with narrow beam geometry and the collimator was attached to a Rad-94 (Varian Medical Systems, Salt Lake City, UT) tungsten-target x-ray tube.

Other studies that compared monochromatic and polychromatic beams for mammography, included the use of Monte Carlo techniques and homogeneous software phantoms mimicking adipose tissue filled with cavities of several materials such as PMMA, glandular tissue and water (Fitousi et al 2012) or software anthropomorphic phantoms (Muller et al 2011). Both studies demonstrated improved contrast and noise characteristics of images acquired with monochromatic beams compared to those using polychromatic spectra. The first study (Fitousi et al 2012) involved several spectra for the polychromatic acquisition, while the second (Muller et al 2011), compared simulated mammograms generated using a Mo/Mo 
$28 \mathrm{kVp}$ spectrum with mammograms obtained using quasi-monochromatic x-rays providing also information on the deposited dose.

These few studies point to the potential of using monochromatic beams with BT and outline the need for a thorough investigation in this field. In general, average glandular doses for a single BT acquisition are similar to a two-view mammography and are reported to vary between 0.6 to 4.0 mGy (Feng and Sechopoulos 2012, Poplack et al 2007, Dobbins 2009). The radiation dose depends not only on the exam itself, but also on whether the tomosynthesis exam will be combined with mammography. As a screening technology, reducing the radiation dose required for $\mathrm{BT}$ acquisition with no loss or even improvement of image quality is always desirable.

A preclinical setup for BT with Synchrotron has been already accomplished at Elettra and was used in our previously reported studies (Bliznakova et al 2010a, Malliori et al 2012) and is analytically described in Chapter 6 . In both cases, the phantom was placed on a stage that moved vertically during each image acquisition and rotated in order to obtain images at each specified angle. The group of the Elettra Synchrotron radiation facility has been dealing with synchrotron mammography for many years. Apart from absorption effects, phase effects in breast imaging have been extensively studied (Arfelli et al 1998a, Arfelli et al 2000, Tromba et al 2011). Presently, at the SYRMEP beamline at Elettra, a synchrotron radiation clinical program in mammography is running (Dreossi et al 2008, Castelli et al 2011, Quai et al 2013, Longo et al 2014). Clinical trials have started since 2006 aiming initially to doubtful cases: women who have undertaken a conventional mammography with an ambiguous diagnosis would be offered the possibility of undergoing synchrotron radiation (SR) mammography. At the beginning only screen-film systems were used, while later computer radiography (CR) systems participated in the clinical trial (Quai et al 2013). For the purposes of the program, in the patient room, the patient lays prone on a high precision movement support, which includes a special opening for the breast with the size and shape of the aperture consistent with the chest anatomy (Fig. 3.5). The breast compression paddles are located under the hole in the support. The movement system is equipped with three motion stages: horizontal for positioning, vertical for scanning and rotational along an axis perpendicular to the beam for oblique breast projection (Abrami et al 2005). This setup may be also applicable for BT. The scan velocity can be accurately selected up to $4 \mathrm{~cm} / \mathrm{s}$. Absorption and in-line phase contrast images can be acquired. Instead of antiscattering grid used in conventional mammography, the system has a slit aligned with the beam in front of the detector holder. 


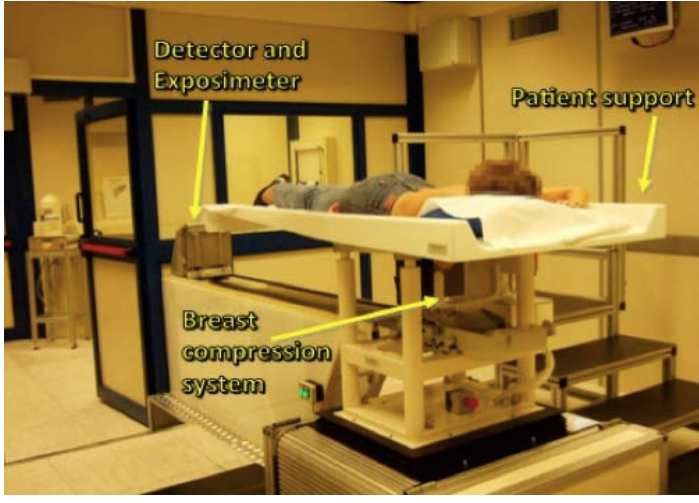

(a)

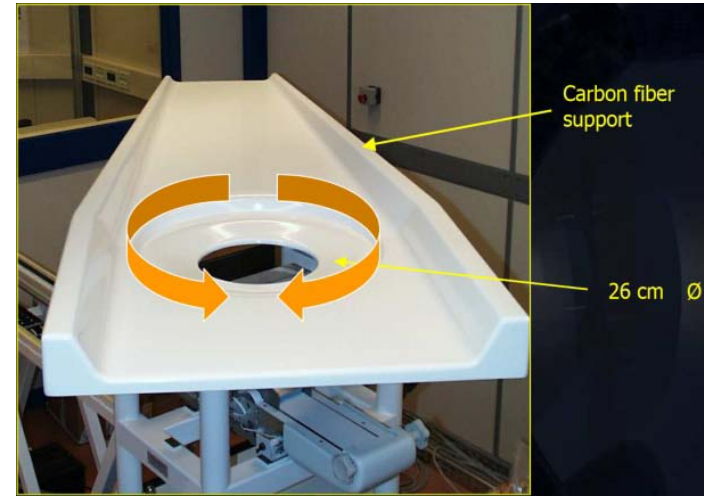

(b)

Fig. 3.5. (a) The patient room of SYRMEP facility at Elettra Synchrotron Laboratory, Trieste (Italy). In particular the Synchrotron mammography setup is presented: the movable support, the breast compression system, the position of detector and exposimeter (image from Fedon et al 2014) (b) Upper view of the patient support (image from Rigon 2008, Proc. of the ICFA Workshop on Compton Sources for X/gamma Rays: Physics and Applications, Alghero, Italy).

However, performing routine diagnostic imaging procedures using synchrotrons can be a complex and expensive procedure. An important limitation factor also, could be resolution loss and possibly motion artifacts due to breast movement during table rotation. Usually acquisition of one projection image takes a few seconds; thus a set of 11 or 15 images may take up to 3-4 minutes. For such a relatively long image acquisition time, avoiding or correcting for motion could be challenging. Although such challenges currently exist, future technological advances could lead to the realization of monochromatic BT imaging systems at synchrotron facilities. 
CHAPTER 4

BREAST TOMOSYNTHESIS COMPUTER-BASED PLATFORM

\section{CHAPTER SUMMARY}

This chapter describes the computer-based platform that was developed for dedicated BT studies. The scope of this platform was to incorporate the MPA and BP reconstruction algorithms, combined with several filters for exploitation of BT performance. The algorithms are compatible with various image acquisition geometries, and have been adapted and presented in detail for partial isocentric rotation with stationary detector, which is the most common acquisition geometry for BT. 


\subsection{INTRODUCTION}

The Biomedical Technology Unit at the University of Patras, has a 25 years experience in developing and optimizing image acquisition geometries and reconstruction algorithms for computed tomography (Badea 2000, Buliev 2004, Kamarianakis et al 2011, Soimu et al 2008) with focus on the specific field of tomosynthesis (Kolitsi et al 1992, Badea et al 2001, Soimu et al 2005, Kolitsi et al 1993, Badea et al 2002, Bliznakova et al 2010a, Messaris et al 1999). For the purposes of the current study, a computer-based platform has been developed in Matlab for tomosynthesis studies dedicated for breast imaging (Malliori et al 2010). The platform incorporates different reconstruction algorithms that have been developed and suitably adjusted for the acquisition geometries used in BT and a number of filters that can be applied before or after the reconstruction of the image in order to remove the noise. A graphical user interface (GUI) has been also developed to further facilitate the use of the tool (Malliori et al 2014). The platform has been exploited to evaluate the quality of BT images reconstructed from simulated projections of tissue-equivalent breast phantoms as well as from experimental data acquired using hardware breast phantoms.

\subsection{THE PLATFORM}

\subsubsection{Design of the computer-based tomosynthesis platform}

A block-diagram of the computer-based platform that has been developed in Matlab (The MathWorks, Inc.) is shown in Fig. 4.1. The BT platform may easily be extended to incorporate more reconstruction algorithms and filters and consists of four parts: Data, Filters, Reconstruction Algorithms and Reconstructed Images. 


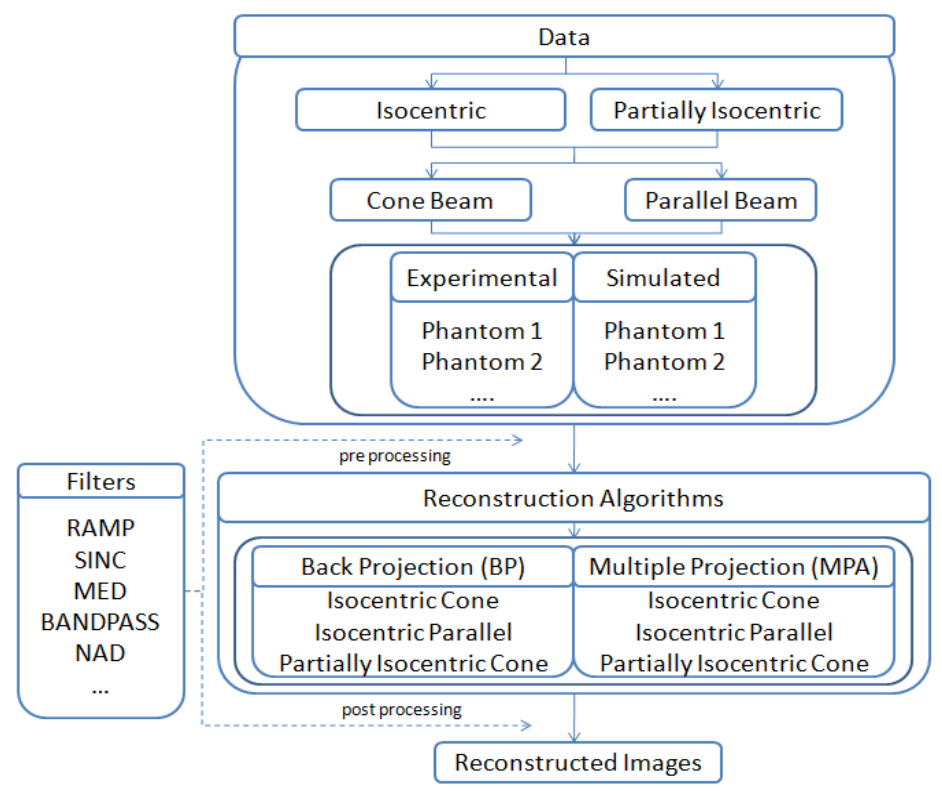

Fig. 4.1. Computer-Based platform for BT.

Reconstruction Algorithms. Two DTS algorithms to obtain the reconstructed volume from the projection images were studied and incorporated into the computer platform: The BP and the MPA. The algorithms have been implemented for isocentric and partial isocentric rotation with stationary detector and can be used with both parallel and cone beams. The basic principles of image formation and equations are analytically presented in sections 4.3 .1 and 4.3.2.

Filters. The image that is produced after the backprojection has a high density in the center because of the different images being overlapped in this area. Filtering can be applied before or after the reconstruction to remove noise and artifacts from the image. Several types of filters can be used aiming to eliminate artifacts in the image and improve the reconstruction quality of high- and low-contrast features such as $\mu$ Cs and masses. In this platform the implemented filters can be applied on the projections prior or after the reconstruction including a simple ramp, a sinc filter, median, bandpass, non-linear anisotropic diffusion (NAD) filters.

Ramp is a commonly used filter prior to backprojection in x-ray tomography and tomosynthesis and has the effect of filtering out the low frequencies and passing the high frequencies with a linear behavior in between.

The Sinc filter, as proposed by Van de Sompel for BT (Van De Sompel et al 2011), is described by the following equation: 


$$
F\left(\omega_{x}\right)=\mid \frac{2}{\alpha} \sin \left(\frac{\alpha \omega_{x}}{2}\right)\left(\frac{\sin \left(\frac{\alpha \omega_{x}}{2}\right)}{\frac{\alpha \omega_{x}}{2}}\right)^{2}
$$

This filter has a varying response depending on the value of parameter $a$. For a value of $a<1$, the sinc filter approaches the standard ramp filter and acts as a simple high-pass filter that is widely used prior to backprojection reconstruction. As $a$ increases, a smoother high-frequency roll-off is achieved.

Median filters result in smoothing of the image without causing excessive blurring or degradation of the edges. Median filtering is an effective technique for removing random noise that appears in the form of black or white pixels on the images and has much better results than other low pass filters for small or moderate levels of noise.

Bandpass filters attenuate high and low frequencies outside a selected frequency range that is preserved. However, there is a region just outside the intended passband where the frequencies are attenuated, but not completely rejected.

Nonlinear Anisotropic Diffusion (NAD) filtering is based on nonlinear evolution partial differential equations $\mathrm{u}$, and seeks to improve images by removing noise while preserving details and even enhancing edges. These filters has been developed based on the diffusion function (Eq. 4.2) as proposed by Perona and Malik (Perona and Malik 1987).

$$
c(x, y)=\exp \left[-0.5\left(\frac{|\Delta u|}{\kappa}\right)^{2}\right]
$$

The NAD filter has been adapted for BT in the framework of this study and is presented in comparison to other filtering techniques (Malliori et al 2014) while the first four filters have been reported in several BT studies (Sone et al 1991, Zhao and Zhao 2008, Lu et al 2010, Samala et al 2014, Van De Sompel et al 2011) and have been used on simulated and experimental BT data acquired at synchrotron facilities (Malliori et al 2010, Malliori et al 2012, Bliznakova et al 2010a, Malliori et al 2013).These filters can be used as postprocessing filters as well, and be applied to the image after the reconstruction of the projections while more filters can also be easily added and incorporated in the platform.

Data. The data is stored in the platform and consists of both parallel and fan beam projections. Both, simulated and experimental data may be used with the developed reconstruction techniques, integrated into the BT platform.

\subsubsection{Graphical User Interface}

To facilitate further research we developed a Graphical User Interface (GUI) for the DTS simulation platform. The BT interface (Fig. 4.2) enables the easy and fast import of 
acquisition and reconstruction parameters, the selection of the reconstruction algorithm and filtering method.

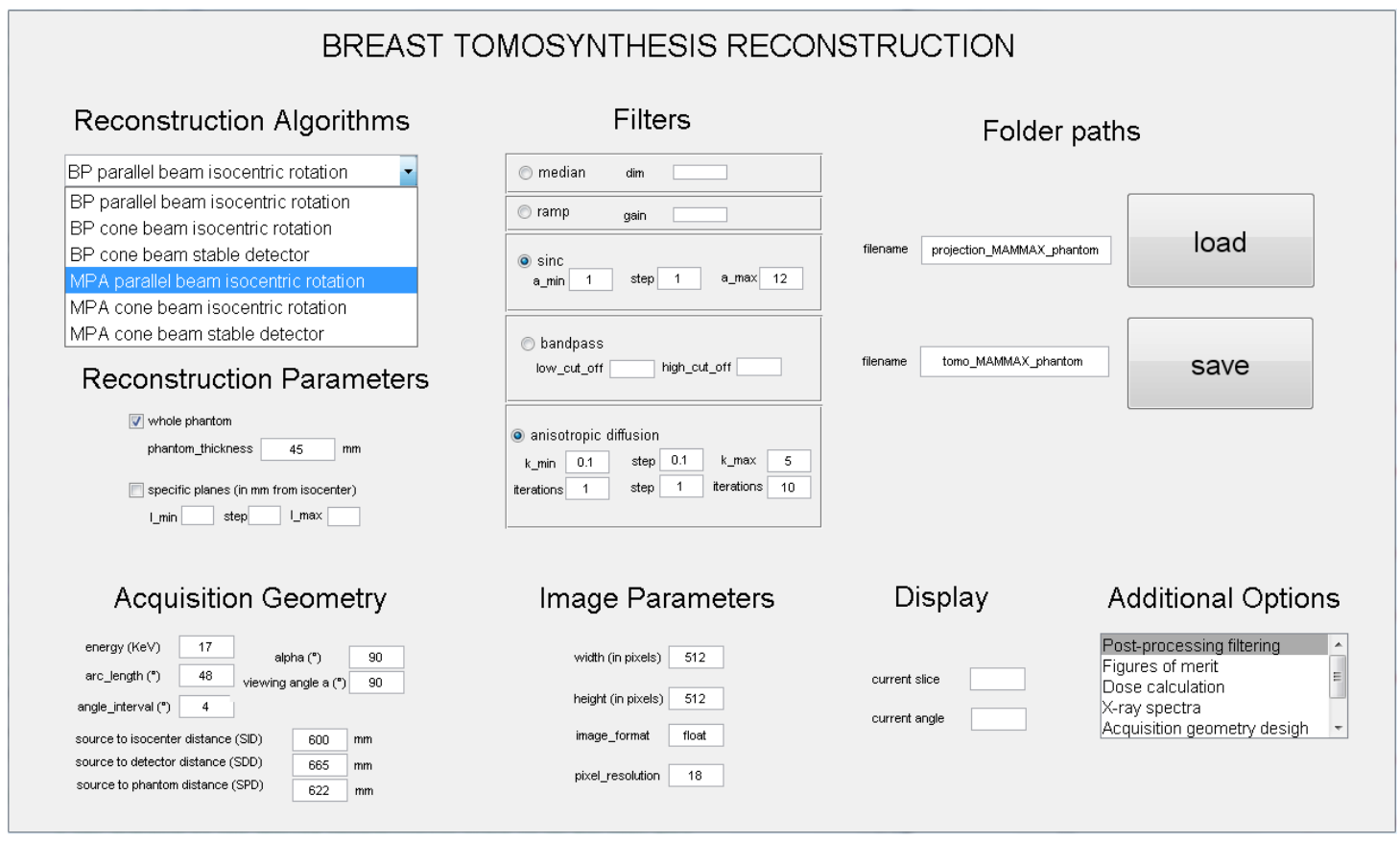

Fig. 4.2. Graphical User Interface of the BT platform.

In short the GUI allows reconstruction of breast images that are simulated or experimentally acquired with both isocentric and partial isocentric rotation (stationary detector) and parallel/cone beam. The Reconstruction Algorithms field includes the BP and MPA algorithms for isocentric rotation (parallel and cone beam) and for partially isocentric rotation (cone beam). These reconstruction algorithms may be combined with any of the filters available for pre-processing of projection images or post-processing of reconstructed tomograms in the Filters field. Parameters concerning the reconstructed tomosynthesis images or the acquisition geometry used for obtaining the projection data can be defined in the Reconstruction Parameters, Acquisition Geometry and Image Parameters fields while the current angle and slice during the reconstruction are shown in the Display field. In Folder paths, the desired folder can be browsed from which the projection data are loaded initially and then the tomosynthesis slices are saved after the reconstruction. In the field Additional Options, post-processing filtering, image evaluation using different figures of merit, image visualization and processing, dose calculation etc. can be accomplished. 


\subsection{BASIC PRINCIPLES AND MATHEMATICAL DESCRIPTION OF THE RECONSTUCTION ALGORITHMS}

\subsubsection{The Multiple Projection Algorithm}

The Multiple Projection Algorithm (MPA) is a DTS reconstruction algorithm of maximized efficiency and is described in details in Kolitsi et al. (Kolitsi et al 1992). Pixels are translated in groups rather than on an individual basis resulting in a multifold reduction of required computing time. This algorithm was initially developed for isocentric rotation and used in simulation studies (Badea et al 2001, Soimu et al 2005, Bliznakova et al 2012a, Malliori et al 2013) and has been further modified and extended for parallel beams and used in BT studies performed at Synchrotron facilities (Bliznakova et al 2010a, Malliori et al 2012). Moreover, it has been suitably adjusted and evaluated for partially isocentric rotation with stationary detector. The algorithms and equations are described analytically in the following sections.

\section{Multiple Projection Algorithm for isocentric rotation}

The principle of image formation for the geometry of isocentric rotation using MPA is illustrated in Fig. 4.3.

In such units the x-ray tube and the image intensifier rotate around a fixed point, the isocenter (I) while maintaining a constant distance between source to isocenter (SID) noted as $b$ and between source to detector (SDD) noted as $d$. In Fig. 4.3, the two positions of the source $S$ and $S^{\prime}$ correspond to a zero angle and an angle equal to $w$. $I A$ and $Q P$ are the intersections of the plane of rotation with the horizontal planes at distance 0 and $\ell$ above the isocenter, respectively.

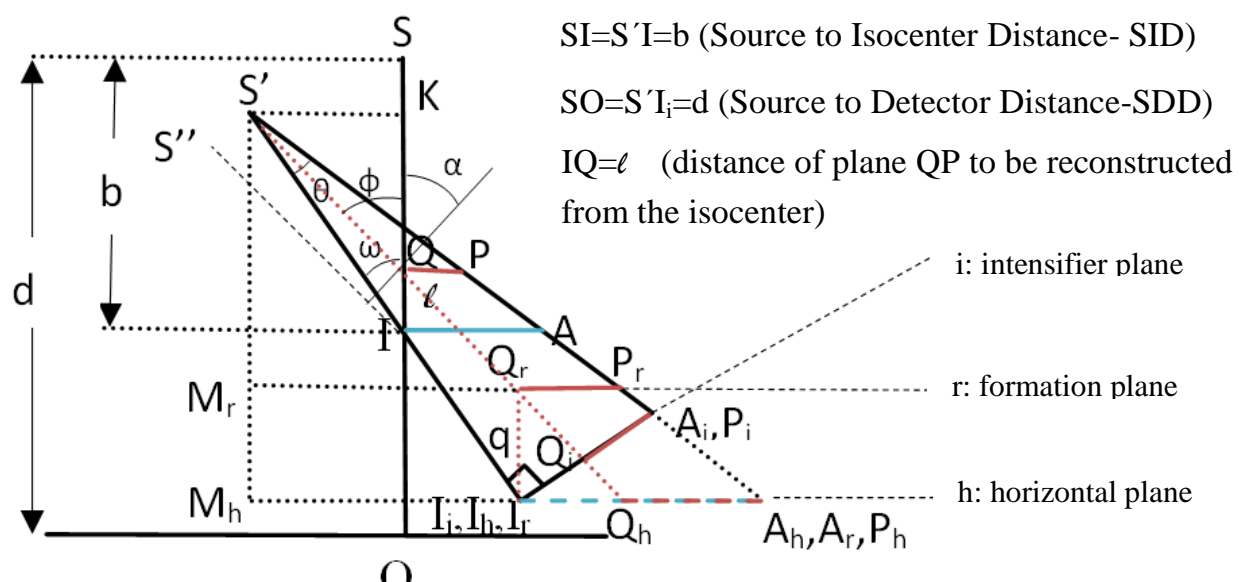

Fig. 4.3. Schematic representation of the principle of image formation in isocentric rotation using MPA 
At an angle $w$, the acquired images on the image intensifier of these two planes will be $I_{i} A_{i}$ and $Q_{i} P_{i}$. The projections of these planes onto the horizontal plane passing through the intersection of the beam's central axis and the intensifier plane are $I_{h} A_{h}$ and $Q_{h} P_{h}$, respectively. In case of IA lying on the plane of the isocenter, the image formation plane is the same as the horizontal plane, thus $I_{r} A_{r}=I_{h} A_{h}$. In order to obtain a focused image in case of QP lying at $\ell$ above the plane of the isocenter, $Q_{h} P_{h}$ should be first horizontally shifted by $I_{h} Q_{h}$ and subsequently normalized to the magnification of the isocenter plane. Here, $Q_{r} P_{r}$ represents the size modified image of $Q P$ at a distance $q$ above the horizontal plane. Addition of these projections, over all viewing angles will yield the desired reconstruction. Thus, in the case of horizontal planes, the image formation plane is a horizontal plane which follows the motion of the x-ray tube. During rotation the source $S$ is moving at a circle of radius $b$ around the isocenter, while the image intensifier at a circle of radius $d-b$ around the same point. The distance $I_{i} Q_{r}$ is constant for a given $\ell$. Therefore, the formation plane is moving also at a circle of radius $d-b$ but with the center displaced by an amount $I_{i} Q_{r}$ equal to $q$

The equations of $h$ and $r$ can be derived as follows. From the triangle $\mathrm{I}_{\mathrm{i}} \mathrm{A}_{\mathrm{i}} \mathrm{A}_{\mathrm{h}}$ :

$$
\frac{I i A i}{\sin [90-(w+\theta)]}=\frac{\operatorname{IhAh}}{\sin (90+\theta)} \text { or } \frac{i}{h}=\frac{\sin [90-(w+\theta)]}{\sin [90+\theta)}=\frac{\cos (w+\theta)}{\cos \omega \theta}=\cos w-\sin w \tan \theta
$$

However,

$$
\tan \theta=\frac{I i A i}{S^{\prime} I i}=\frac{i}{d}
$$

Thus Eq. 4.3 becomes:

$$
\mathrm{h}=\frac{\mathrm{di}}{\mathrm{d} \cos w-i \sin w}
$$

Equation 4.5, describes the position $h$ onto the horizontal plane that the acquired column $i$ is projected on. In this case, that the plane to be reconstructed (IA) lies on the plane of the isocenter, the image formation plane $r$ coincides with the horizontal $h$.

In case the plane to be reconstructed is above or below the isocenter at a distance $\ell$ such as plane $Q P$, then the image will be formed at some distance $q$ from the isocenter. Specifically, $q$ is the distance $I_{h} Q_{r}$ of the image formation plane from the horizontal plane, through $I_{i}$. From the similar triangles $S^{\prime} I Q$ and $S^{\prime} I_{h} Q_{r}$ :

$$
q=\frac{l d}{b}
$$

Similarly, from triangles $S^{\prime} Q_{r} P_{r}$ and $S^{\prime} Q_{h} P_{h}$ : 


$$
\frac{Q r P r}{Q h P h}=\frac{S^{\prime} Q r}{S^{\prime} Q h}=\frac{S^{\prime} M r}{S^{\prime} M h}=\frac{S^{\prime} M h-q}{S^{\prime} M h}=1-\frac{q}{d \cos w}=1-\frac{l}{b \cos w}
$$

The amount of shift $\left(I_{h} Q_{h}\right)$ required to bringing $Q_{h}$ at the central column of the horizontal matrix is:

$$
I_{h} Q_{h}=q \tan \varphi
$$

From triangle $S^{\prime} K Q$ :

$$
\tan \varphi=\frac{S^{\prime} K}{K Q}=\frac{S^{\prime} K}{K I-l}=\frac{b \sin w}{b \cos w-l}
$$

Thus, the shift becomes:

$$
I_{h} Q_{h}=q \frac{b \sin w}{b \cos w-l}=\frac{l d \sin w}{b \cos w-l}
$$

The position of the column containing the image of $P$ on the image formation plane $r$, according to Eqs. 4.7 and4.10, is given by the expression:

$$
r=Q r P r=Q h P h\left(1-\frac{l}{b \cos w}\right)=(h-I h Q h)\left(1-\frac{l}{b \cos w}\right)=\left(h-\frac{l d \sin w}{b \cos w-l}\right)\left(1-\frac{l}{b \cos w}\right)
$$

Equation 4.5 describes the position $h$ of the horizontal plane that the column $i$ of the acquired image is projected on, whereas Eq. 4.11 describes the position $r$, that this column is moved to on the image formation plane.

\section{Multiple Projection Algorithm for partial isocentric rotation (stationary detector)}

The principle of image formation for the geometry of partially isocentric rotation using MPA is illustrated in Fig. 4.4.

In such units the x-ray tube rotates around the isocenter (I), maintaining a constant distance between source to isocenter noted as $b$ while the detector is remained stationary. The distance between source to detector is changed with the angle $w$. The distance between source to detector (SDD) is changing with the angle $w$. At zero degrees $(w=0)$ the distance is equal to $d$. In the general case the distance is equal to $d^{\prime}=b+\frac{d-b}{\cos w}$.

The two positions of the source $S$ and $S^{\prime}$ (Fig. 4.4) correspond to zero angle and an angle equal to w. $I A$ and $Q P$ are the intersections of the plane of rotation with the horizontal planes at distance 0 and $l$ above the isocenter, respectively. Subscript $s$ symbolizes the position of column in the acquired image on the stationary detector. At an acquisition angle $w$, the 
acquired images on the image intensifier of $I A$ and $Q P$ will be $I_{s} A_{s}$ and $Q_{s} P_{s}$. Subscripts $h^{\prime}$ and $r$ ' show positions on the horizontal and formation planes, respectively, while subscripts $h$ and $r$, show the corresponding former positions of the horizontal and formation planes in case of isocentric rotation with rotating source-detector.

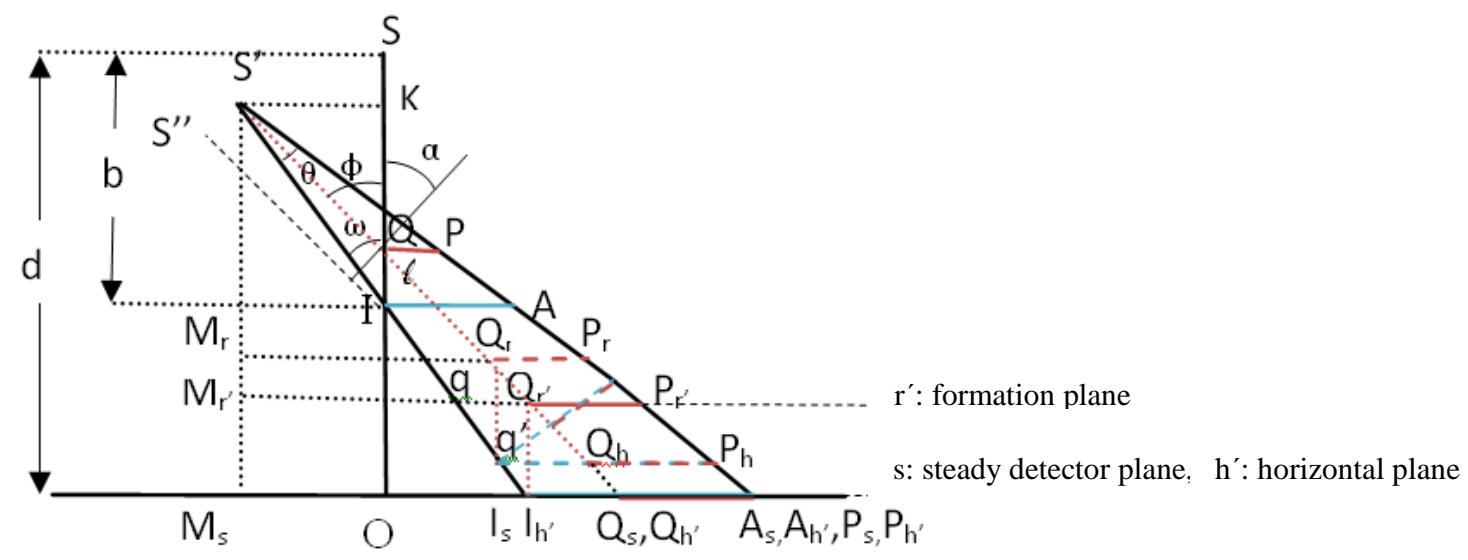

Fig. 4.4. Schematic representation of the principle of image formation for partially isocentric rotation with stationary detector using MPA.

The horizontal plane $h^{\prime}$ formed by the beam's central axis and the intensifier plane, in this case of stationary detector, is at the level of the detector $s$ shifted by $O I_{s}=(d-b) \tan w$. The projections of $I A$ and $Q P$ on the horizontal plane are $I_{h} \cdot A_{h}$, and $Q_{h} P_{h^{\prime}}$, respectively. In case of $I A$ lying at the plane of the isocenter, the image formation plane is the same as the horizontal plane thus $I_{r} \cdot A_{r}=I_{h} \cdot A_{h}$. In order to obtain a focused image in case of $Q P$ lying at 1 above the plane of the isocenter, $Q_{h^{\prime}} P_{h^{\prime}}$ should be shifted horizontally by $I_{h^{\prime}} Q_{h^{\prime}}$, and subsequently normalized to the magnification of the isocenter plane resulting in $Q_{r^{\prime}} P_{r^{\prime}}$.

More specifically, to obtain the image at $h^{\prime}$ describing the position of the horizontal plane that the column $s$ of the acquired image is projected on, should be shifted by $\mathrm{OI}_{s}$ :

$$
h^{\prime}=s-(d-b) \tan w
$$

In order to obtain the position that this column is moved to on the image formation plane $r^{\prime}$, we need to use the same formulas as for the MPA in complete isocentric rotation, replacing $d$ with $d^{\prime}$. Therefore, $r^{\prime}$ is calculated as:

$$
r^{\prime}=\left(h^{\prime}-\frac{l d^{\prime} \sin w}{b \cos w-l}\right)\left(1-\frac{l}{b \cos w}\right)
$$

where: 


$$
d^{\prime}=S^{\prime} I_{s}=b+\frac{d-b}{\cos w}
$$

Equation 4.13 can be analytically derived as follows:

The distance between the image formation plane and the horizontal plane noted as $q$ ' is equal to $I_{h}{ }^{\prime} Q_{r}{ }^{\prime}$. From the similar triangles $S^{\prime} I Q$ and $S^{\prime} I_{h}{ }^{\prime} Q_{r}{ }^{\prime}$ :

$$
q^{\prime}=\frac{l d^{\prime}}{b}
$$

and from the triangle $S^{\prime} K Q$ :

$$
\tan \varphi^{\prime}=\frac{S^{\prime} K}{K Q}=\frac{S^{\prime} K}{K I-l}=\frac{b \sin w}{b \cos w-l}
$$

The amount of shift $\left(I_{h}, Q_{h}{ }^{\prime}\right)$ required to bring $Q_{h}{ }^{\prime}$ at the central column of the horizontal plane is:

$$
I_{h}^{\prime} Q_{h}^{\prime}=q^{\prime} \tan \varphi^{\prime}=\frac{l d^{\prime}}{b} \frac{b \sin w}{b \cos w-l}=\frac{l d^{\prime} \sin w}{b \cos w-l}
$$

From triangles $S^{\prime} Q_{r}, P_{r}$, and $S^{\prime} Q_{h}, P_{h}{ }^{\prime}$ :

$$
\frac{Q r^{\prime} P r^{\prime}}{Q h^{\prime} P h^{\prime}}=\frac{S^{\prime} Q r^{\prime}}{S^{\prime} Q h^{\prime}}=\frac{S^{\prime} M r^{\prime}}{S^{\prime} M s}=\frac{S^{\prime} M s-q^{\prime}}{S^{\prime} M s}=1-\frac{q^{\prime}}{d^{\prime} \cos w}=1-\frac{d^{\prime} l}{b d^{\prime} \cos w}=1-\frac{l}{b \cos w}
$$

Thus, using the shift from Eq. 4.17 and the magnification expressed in Eq. 4.18, the position of $r^{\prime}$ (Eq. 4.13) is derived:

$$
r^{\prime}=\left(h^{\prime}-\frac{l d^{\prime} \sin w}{b \cos w-l}\right)\left(1-\frac{l}{b \cos w}\right)
$$

or by replacing $d^{\prime}$ 'we obtain $r$ for different distances $l$ from isocenter ( $l$ is positive if reconstructed plane is above isocenter and negative if below):

$$
r^{\prime}=\left(h^{\prime}-\frac{l\left(b+\frac{d-b}{\cos w}\right) \sin w}{b \cos w-l}\right)\left(1-\frac{l}{b \cos w}\right)=\left(h^{\prime}-\frac{\operatorname{ltan} w(b \cos w+d-b)}{b \cos w-l}\right)\left(1-\frac{l}{b \cos w}\right)
$$

Equation 4.12 describes the position $h^{\prime}$ of the horizontal plane, whereas Eq. 4.19 describes the position $r^{\prime}$, that this column is moved to on the image formation plane.

\subsubsection{The Backprojection algorithm}

The Back Projection algorithm (BP) is equivalent to Shift-and-add tomosynthesis method and enables the reconstruction of arbitrary planes of any direction relatively to the fulcrum plane. In geometries where the tube is moving during the acquisition, the structures which belong to planes other than the fulcrum plane appear at different positions on the detector. 
This displacement is compensated by shifting the acquired images to an amount defined by geometry. Then the shifted images are added, so that objects of the same plane will appear in focus and structures in other planes will appear blurred.

\section{Backprojection for partial isocentric rotation (stationary detector)}

The principle of image formation for the geometry of partially isocentric rotation using backprojection is illustrated in Fig. 4.5.

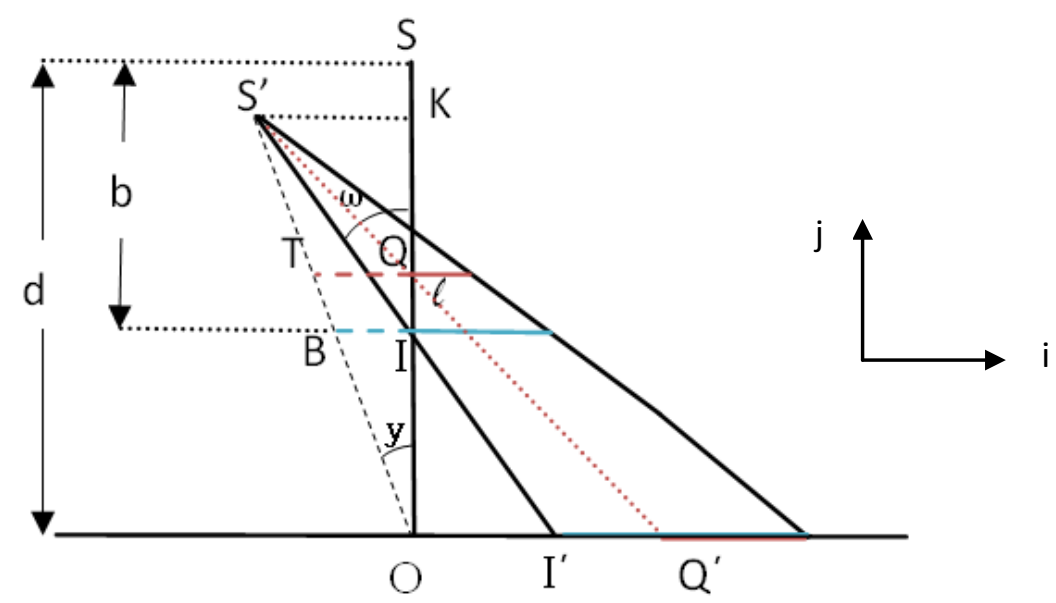

Fig. 4.5. Schematic representation of the principle of image formation for partially isocentric rotation with stationary detector.

In such units the magnification is not constant and changes with angle $w$. Points $I(i, j)$ lying on the level of the isocenter, are projected on $I^{\prime}\left(i^{\prime}, j^{\prime}\right)$. The magnification in this case, is equal to:

$$
R_{0}=\frac{K O}{K I}=\frac{K I+I O}{K I}=\frac{s^{\prime} I \cos w+d-b}{S^{\prime} I \cos w}=\frac{b \cos w+d-b}{b \cos w}
$$

The shifting required to be performed on the columns (i) of this row is equal to:

$$
\text { Shift }_{0}=B I=(d-b) \tan y
$$

But,

$$
\tan y=\frac{b \sin w}{b \cos w+d-b}
$$

where $y$ is the angle between the source and the detector surface.

Thus, 


$$
\text { Shift }=(d-b) \frac{b \sin w}{b \cos w+d-b}
$$

In the general case that a point lies at a distance $l$ above or below the isocenter plane such as $Q(i, j)$ and is projected on the detector plane at $Q^{\prime}\left(i^{\prime}, j^{\prime}\right)$, then the magnification is equal to:

$$
R=\frac{K O}{K Q}=\frac{b \cos w+d-b}{b \cos w-l}
$$

and the shifting is equal to:

$$
\text { Shift }=T Q=(d-b+l) \tan y=(d-b+l) \frac{b \sin w}{b \cos w+d-b}
$$

The equations for $i$ and jare:

$$
\begin{gathered}
i=i^{\prime} \frac{1}{R}=i^{\prime} \frac{b \cos w-l}{b \cos w+d-b} \\
j=j^{\prime} \frac{1}{R}+\text { Shift }=j^{\prime} \frac{b \cos w-l}{b \cos w+d-b}+(d-b+l) \frac{b \sin w}{b \cos w+d-b}
\end{gathered}
$$

For each point $Q(i, j)$ from backprojection of the projected points $Q^{\prime}\left(i^{\prime}, j^{\prime}\right)$ on the detector, over all viewing angles and summing we can reconstruct the initial volume.

\subsection{INITIAL TESTING USING A SOFTWARE BREAST PHANTOM}

\subsubsection{Phantom and acquisition parameters}

The computer platform was used to evaluate the quality of reconstructed images from simulated projections of tissue-equivalent breast phantoms using DTS. A software version of the CIRS model 011A breast phantom (manufactured by Computerized Imaging Reference Systems, Inc., Norfolk, VA) has been designed (Fig.4.6) with the phantom modelling module of the XRayImagingSimulator (Bliznakova 2003). The ROIs with the details that were studied are shown in Fig.4.6. ROIs I and II represent ROIs with low contrast objects, while ROI III contains two clusters with the smallest $\mu$ Cs (groups 2 and 3). The linear attenuation coefficients of the studied details and their elemental composition are reported in Table 4.1. 


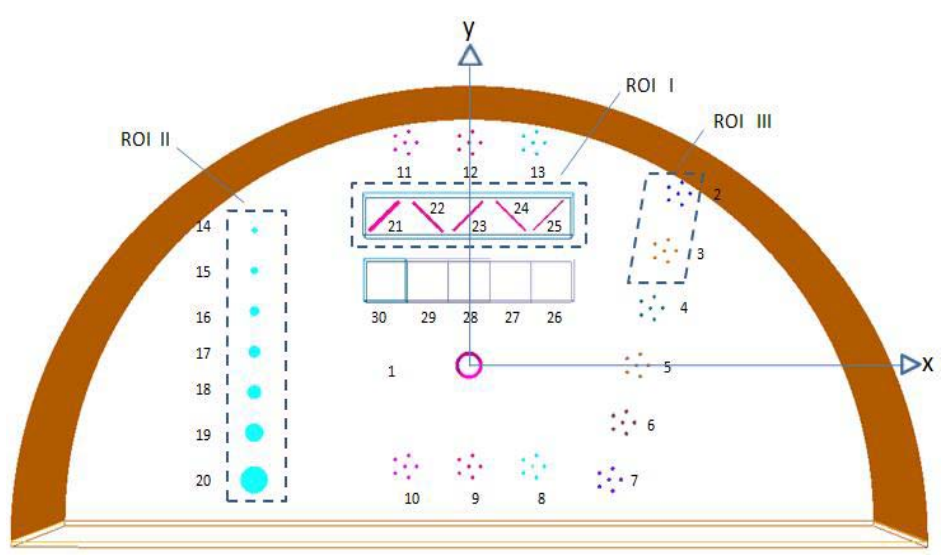

Fig. 4.6. CIRS Breast Phantom.

Synthetic images of the modelled CIRS phantom were generated with isocentric rotationbased DTS system using the image modelling module of the XRayImagingSimulator (Lazos et al 2003, Bliznakova 2003).

Table 4.1. Elemental composition and attenuation coefficients for the simulated tissues

\begin{tabular}{|c|c|c|c|c|}
\hline & Object & $\begin{array}{c}\text { Tissue } \\
\text { composition }\end{array}$ & $\begin{array}{c}\text { Thickness, } \\
\text { mm }\end{array}$ & $\begin{array}{l}\text { Attenuation } \\
\text { coefficient, } \mathbf{c m}^{-1}\end{array}$ \\
\hline 1. & $\begin{array}{c}\text { Basic } \\
\text { phantom }\end{array}$ & $\begin{array}{l}50 \% \text { adipose } 50 \% \\
\text { gland }\end{array}$ & 45.00 & 0.7250 \\
\hline 2. & \multirow{12}{*}{$\begin{array}{l}13 \text { groups of } \\
\mathrm{CaCO}_{3} \text { specs }\end{array}$} & \multirow{12}{*}{$\mathrm{CaCO}_{3}$} & 0.130 & \multirow{12}{*}{1.845} \\
\hline 3. & & & 0.165 & \\
\hline 4. & & & 0.196 & \\
\hline 5. & & & 0.230 & \\
\hline 6. & & & 0.275 & \\
\hline 7. & & & 0.400 & \\
\hline 8. & & & 0.230 & \\
\hline 9. & & & 0.196 & \\
\hline 10. & & & 0.165 & \\
\hline 11. & & & 0.230 & \\
\hline 12. & & & 0.196 & \\
\hline 13. & & & 0.165 & \\
\hline 14. & \multirow{7}{*}{$\begin{array}{l}\text { Hemispheric } \\
\text { masses }\end{array}$} & \multirow{7}{*}{$\begin{array}{l}75 \% \text { gland } \& \\
25 \% \text { adipose }\end{array}$} & 0.90 & \multirow{7}{*}{0.8275} \\
\hline 15. & & & 1.19 & \\
\hline 16. & & & 1.59 & \\
\hline 17. & & & 1.98 & \\
\hline 18. & & & 2.38 & \\
\hline 19. & & & 3.16 & \\
\hline 20. & & & 4.76 & \\
\hline 21. & \multirow{5}{*}{ Nylon fibers } & \multirow{5}{*}{$\left(\mathrm{C}_{6} \mathrm{H}_{11} \mathrm{ON}\right)_{\mathrm{n}}$} & 1.25 & \multirow{5}{*}{0.6546} \\
\hline 22. & & & 0.83 & \\
\hline 23. & & & 0.71 & \\
\hline 24. & & & 0.53 & \\
\hline 25. & & & 0.30 & \\
\hline
\end{tabular}


The simulated imaging protocol used 21 synthetic images acquired at $2^{\circ}$ increments from $-20^{\circ}$ to $20^{\circ}$ using isocentric movement (Fig. 4.3). The distances: source-to-object and sourceto-detector are $1000 \mathrm{~mm}$ and $1300 \mathrm{~mm}$, respectively. Monochromatic parallel beams of 19 $\mathrm{keV}$ are simulated. The detector pixel resolution was set to $0.05 \mathrm{~mm}$, while the image size was 4096 x 4096 pixels.

\subsubsection{Results and conclusions}

Six reconstructed volumes were created using the two reconstruction algorithms BP and MPA with unfiltered and filtered (with ramp and sinc filters, $\alpha=0.1$ ) projection images. Fig. 4.7 shows the whole reconstructed image at a plane where all objects are in focus using MPA algorithm. Similar results are observed for the case of BP.

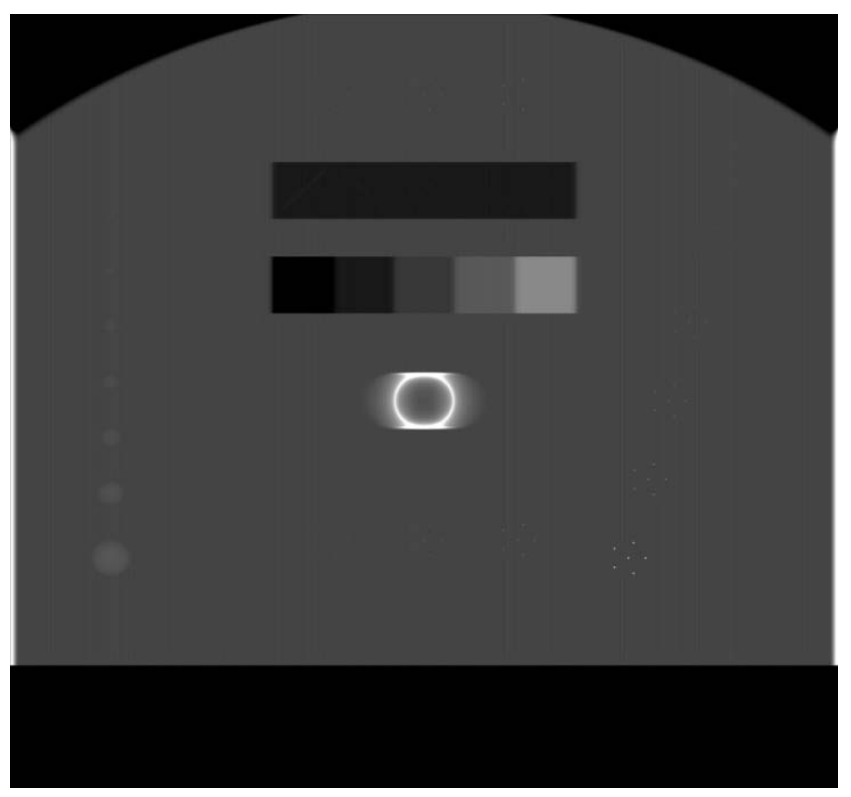

Fig. 4.7. Reconstructed Image of the in-focus plane of the phantom.

To quantitatively evaluate the feature visibility of the reconstructed objects, CNR was calculated for the low and high contrast features at their in-focus plane in the reconstructed slices. The mean and standard deviation values of the background were measured in a $100 \mathrm{x}$ 100 pixel ROI near the objects under evaluation. The corresponding ROI selected for the features of interest depended on their size. The smallest area was that of a microcalcification in a 3x3-pixel area located approximately at its centre. The CNR values using BP and MPA for features belonging to the selected ROIs are presented in Table 4.2. 
Table 4.2. Contrast to Noise Ratio (CNR)

\begin{tabular}{lcc}
\hline & BP & MPA \\
\hline Group 2 (0.165mm) & 14.76 & 14.83 \\
Group $3(\mathbf{0 . 1 9 6 m m )}$ & 29.86 & 25.50 \\
\hline Mass $14(\mathbf{0 . 9 0 m m})$ & 1.85 & 1.80 \\
Mass $20(4.76 \mathrm{~mm})$ & 7.67 & 7.60 \\
\hline
\end{tabular}

Figures 4.8 and 4.9 show the reconstructed ROIs I and II with low contrast objects, while Fig. 4.10 shows the reconstructed ROI III that contains the two groups of $\mu \mathrm{Cs}$.
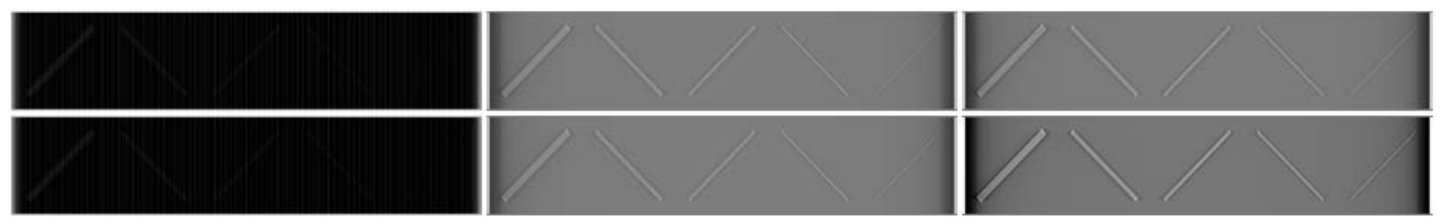

Fig. 4.8. Reconstructed Images of low contrast ROI I-Nylon Fibers; upper row from left to right: BP_UNF, BP_RAMP, BP_SINC and lower row from left to right: MPA_UNF, MPA_RAMP, MPA_SINC.
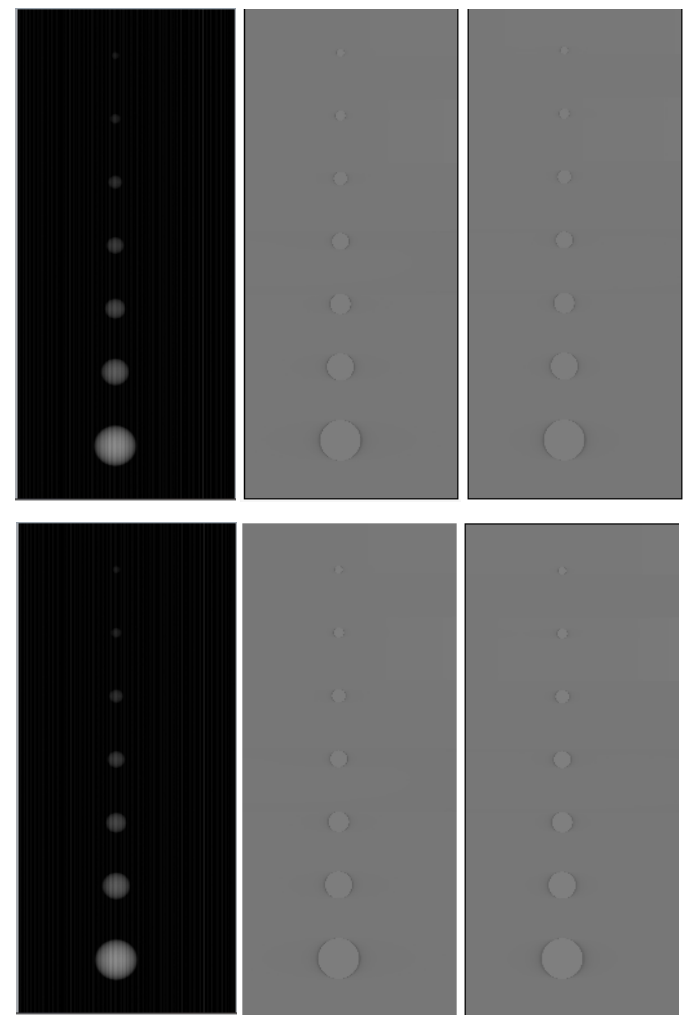

Fig. 4.9. Reconstructed Images of low contrast ROI II - Hemispheric Masses 14-20; upper row from left to right: BP_UNF, BP_RAMP, BP_SINC and lower row from left to right: MPA_UNF, MPA_RAMP, MPA_SINC. 
The images in this figures are reconstructed with BP (upper row) and MPA (lower row) using unfiltered projections in the first column, while the images in the second and third columns are reconstructed with filtered projections using ramp and sinc filters, respectively. For visualization purposes, the images were mapped to 256 grey levels and displayed using the same gray scale window setting. In case of using filtered projections the images are shown with a narrower window to allow better visualization of the objects.

The visual assessment of the images reconstructed with MPA and BP, shows similar reconstruction quality for both low- and high-contrast objects. This is also supported by the quantitative evaluation of these two methods (Table 4.2), showing similar results for the CNR. Therefore, we could conclude that MPA is equivalent to the backprojection algorithm. However, in cases where reconstructions are needed in real time, the MPA is a preferable algorithm due to its significantly higher efficiency concerning the computational time compared to the BP algorithm.

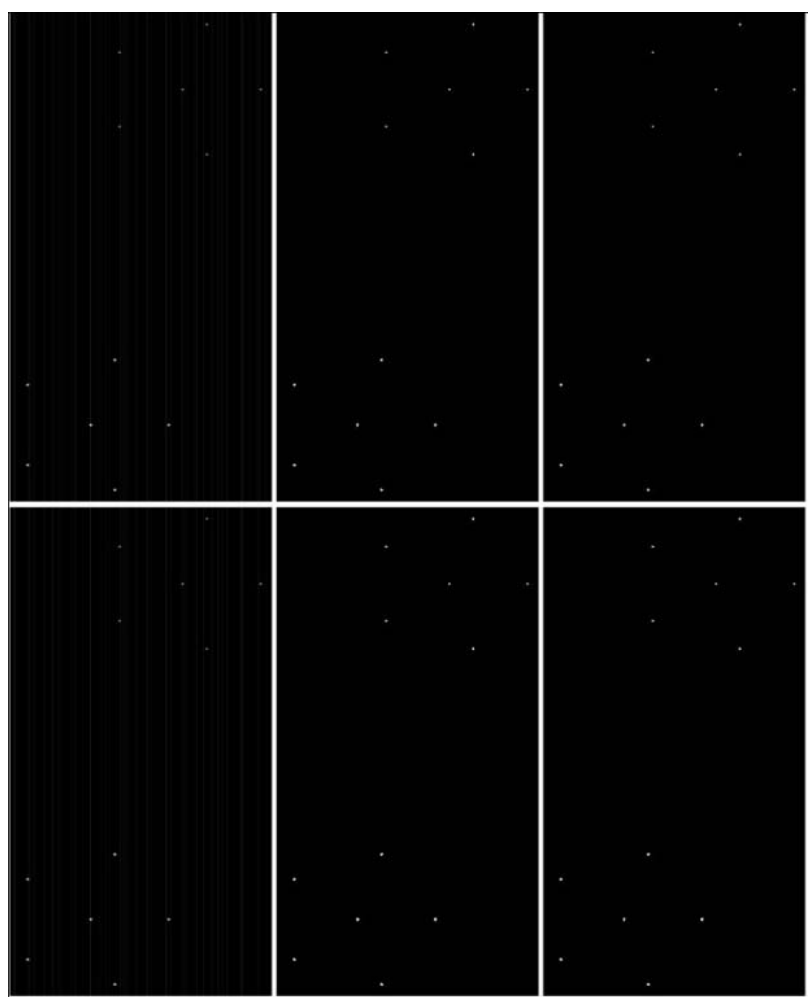

Fig. 4.10. Reconstructed Images of high contrast ROI III - Groups 2 and 3 of $\mathrm{CaCo}_{3}$ specs; upper row from left to right: BP_UNF, BP_RAMP, BP_SINC and lower row from left to right: MPA_UNF, MPA_RAMP, MPA_SINC.

In case as of high contrast features, pre-filtering of the projection images results in better reconstructions. Both filters have the effect of filtering out low frequencies and passing high frequencies, with a linear behavior in between. For this reason, contrasting features such as the $\mathrm{CaCo}_{3}$ groups are accentuated, while blurring is minimized. 
In case of low contrast features, the algorithms that use the filtered projections result in features with sharper edges (Fig. 4.8, Fig. 4.9).

\subsection{INITIAL TESTING USING A HARDWARE BREAST PHANTOM: APPLICATION OF NON-LINEAR ANISOTROPIC DIFFUSION FILTERING}

The tomosynthesis platform has been also exploited to optimize the parameters of a NAD filter adapted for a BT application using real projection images acquired at Elettra Synchrotron facilities. A monochromatic beam of $17 \mathrm{keV}$ was used and 13 projection images were acquired for an arc length of $48^{\circ}$. The feature evaluated, was a $6 \mathrm{~mm}$ low-contrast mass inside a $4.5 \mathrm{~cm}$ thick breast phantom with highly heterogeneous background. In this study, several combinations of the filter (contrast) parameter $\kappa$ for different number of iterations were tested in order to reduce the noise in the image and at the same time improve the visualization of the feature of interest. .Figure 4.11 shows the CNR and C graphs of the $6 \mathrm{~mm}$ mass for different $\kappa$ values (0.01-0.7) and number of iterations (5-40).

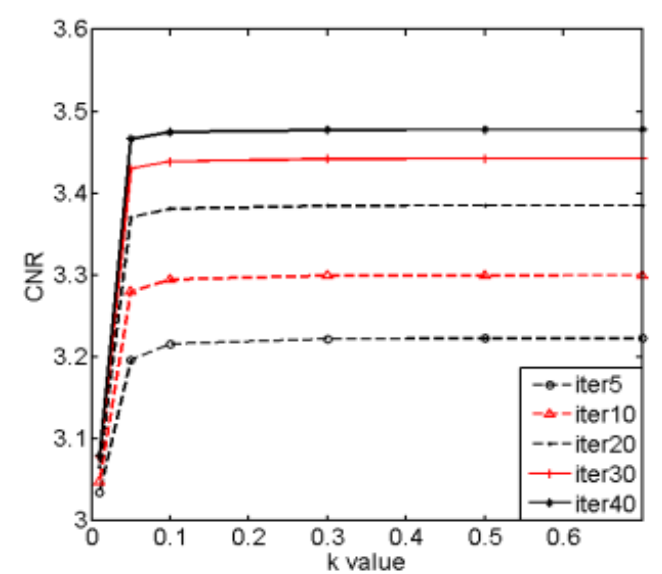

(a)

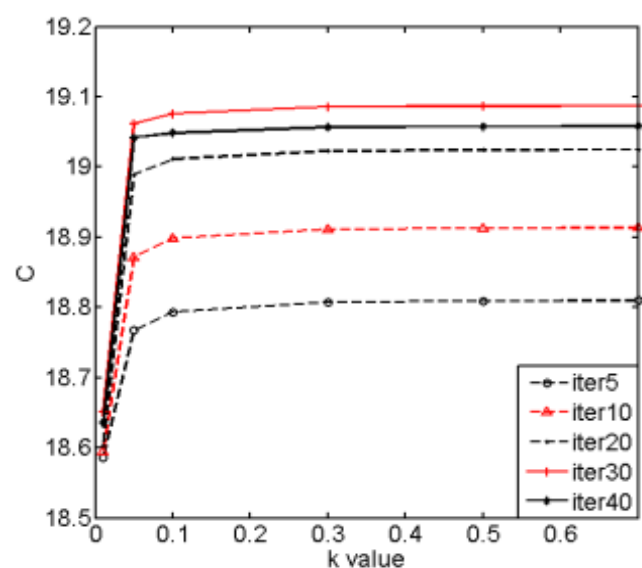

(b)

Fig. 4.11. CNR and C graphs of the $6 \mathrm{~mm}$ mass for different combination of the parameters of the NAD filter.

For the feature under evaluation, there was a progressive improvement observed both visually and quantitatively in the range of 0.01-0.1 and then a much slower improvement up to $\kappa=0.5$. This value was selected for $\kappa$, as it resulted in optimal mass visibility and maximum CNR and $\mathrm{C}$ measurements (Fig. 4.11). Beyond this $\kappa$ value, there was no further improvement in the figures of merit or the feature visualization. A subsequent increase in CNR and C was also observed with increasing the number of iterations. A selection of 30 iterations resulted in 
optimal mass visibility and a good trade between CNR and C values. Further increase of iterations (iter $>30$ ), caused blurring of the image, which was visually observed and quantitatively demonstrated by a reduction of the image C [Fig. 4.11 (b)].

Figures 4.12(a-c) show tomosynthesis slices of the breast mass, reconstructed with MPA applied for unfiltered [Fig. 4.12(a)] and filtered projection images and the corresponding mass profiles below each image. Figure 4.12(b) depicts a tomogram obtained with filtered projection images using a median filter (FMPA_M) while Fig. 4.12(c) using nonlinear anisotropic diffusion filtering (FMPA_NAD) for the selected values of $\kappa=0.5$ and 30 iterations. The subjective comparison shows that the FMPA_NAD demonstrates better low contrast appearance and detection compared to the other two. An improved mass profile was also achieved [Fig. 4.12(c)] indicating noise reduction and a good combination of image smoothing and sharp feature boundaries.
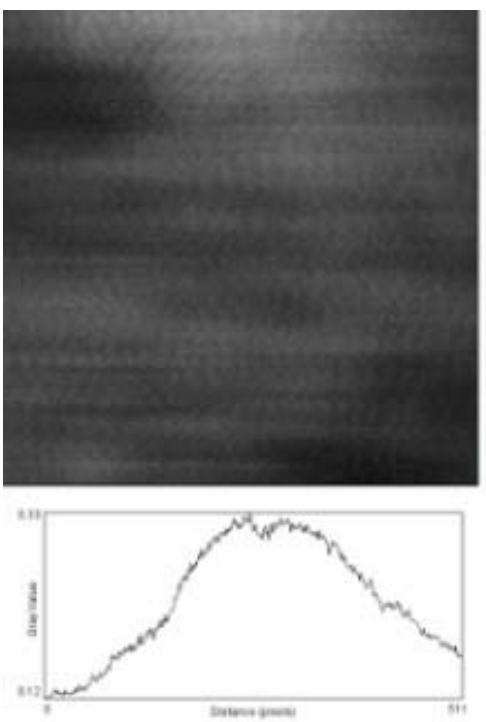

(a)
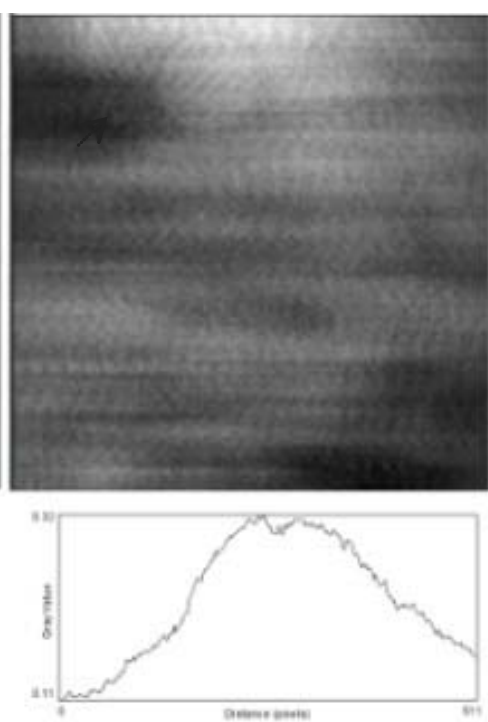

(b)
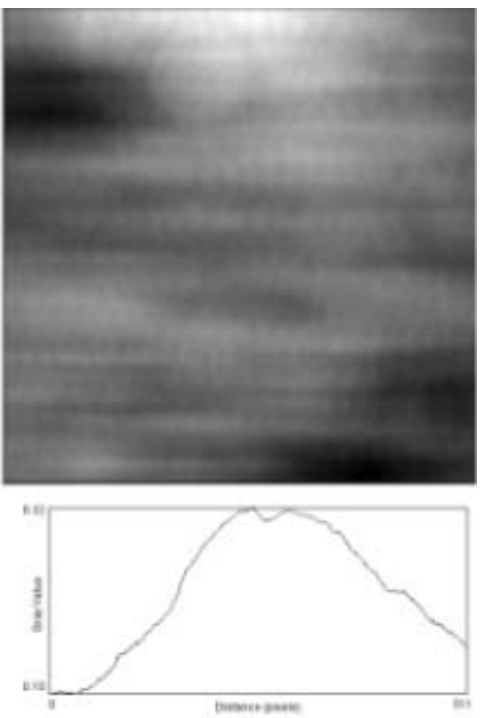

(c)

Fig. 4.12. Tomosynthetic images reconstructed with (a) simple (no filter applied) MPA, (b) filtered with a median filter (FMPA_M) and (c) with NAD (FMPA_NAD) and the corresponding profiles of the $6 \mathrm{~mm}$ mass.

A comparison between CNR and C values, calculated for the $6 \mathrm{~mm}$ low-contrast feature reconstructed with the use of different filters is summarized in Table 4.3. For FMPA_NAD the optimized values of $\kappa=0.5$ and 30 iterations were used while for the case of FMPA_NAD_S, where a sinc filter (Eq. 4.1) was used after NAD, the optimal $\alpha$ parameter for low-contrast features reported in our previous work, i.e. $\alpha=12$ was considered(Malliori et al 2012).Highest CNR was achieved using F_MPA_NAD and FMPA_NAD_S, while the later resulted into an improvement of the $\mathrm{C}$ value by 3 times compared to simple MPA (Table 
4.3). As expected, the CNR of the low-contrast mass is lowest for the case of using ramp prior to MPA. Ramp is a high-pass filter that can enhance the edges and increase $\mathrm{C}$ but also allows the high frequency noise in the image to remain while median is a smoothing filter that can improve the visualization of low contrast features such as breast masses. Sinc filter can be adjusted to behave as high- or low-pass and when used along with NAD can remove the noise while preserving the features' boundaries.

Table 4.3. CNR and C values using different filtering methods in combination with MPA

\begin{tabular}{lll}
\hline Filtering method & CNR & C \\
\hline - (simple MPA) & 2.64 & 18.15 \\
Ramp (FMPA_R) & 0.10 & 41.15 \\
Median (FMPA_M) & 3.09 & 18.60 \\
Anisotropic Diffusion (FMPA_NAD) & 3.44 & 19.08 \\
Anisotropic Diffusion and sinc (FMPA_NAD_S) & 3.25 & 63.13 \\
\hline
\end{tabular}

Figure 4.13 shows a set of tomograms of the mass reconstructed with MPA and filtered with ramp and sinc (first row) for $\alpha=6$ and $\alpha=12$ and combinations of using NAD filter prior to ramp and sinc (FMPA_NAD_R, FMPA_NAD_S) with 5 iterations (second row) and 30 iterations (third row), respectively. NAD carried out with more processing iterations (third row) results in smoothing of the image and suppression of superimposed background. The combination with sinc filter (FMPA_NAD_S) improves the visualization of the mass under evaluation and enables better detection of a second mass (upper right corner) with much lower x-ray contrast. 


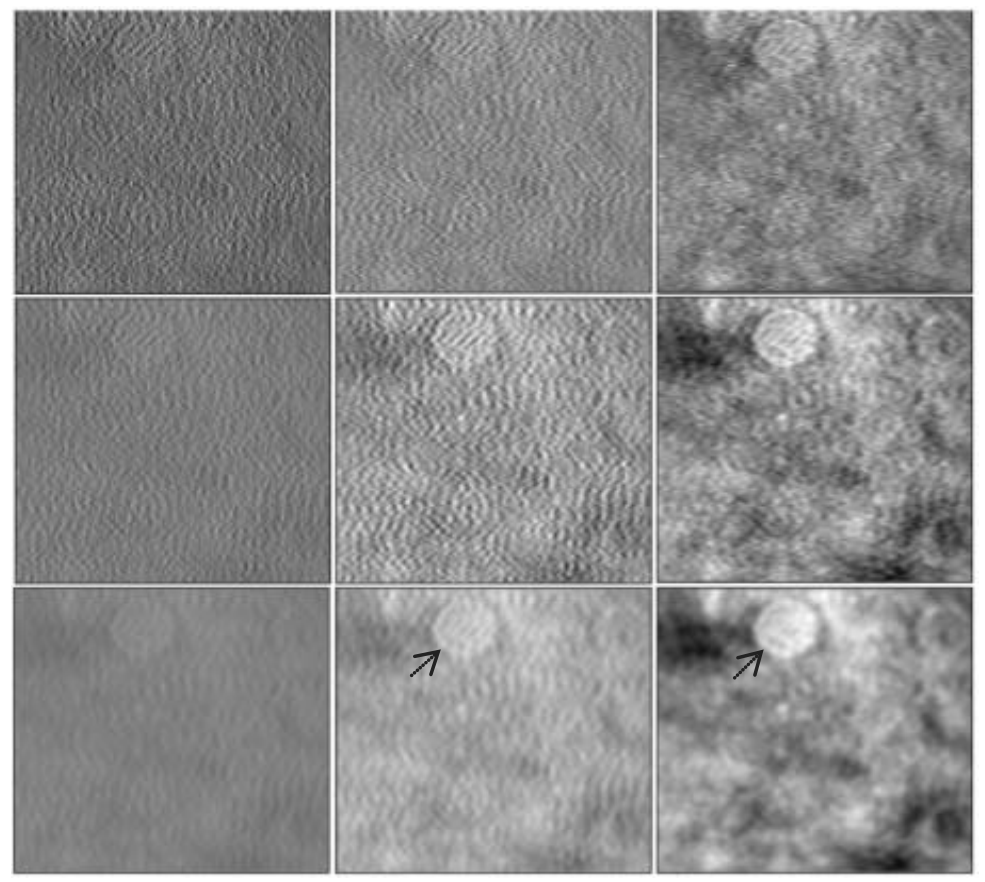

(a)

(b)

(c)

Fig. 4.13. Tomosynthetic images of the $6 \mathrm{~mm}$ mass filtered with: (a) ramp filter (first row), ramp filter after NAD with $\kappa=0.5$ and 5 iterations (second row), ramp filter after NAD with $\kappa=0.5$ and 30 iterations (third row), (b) sinc filter with $\alpha=6$ (first row), sinc filter with $\alpha=6$ after NAD with $\kappa=0.5$ and 5 iterations (second row), sinc filter with $\alpha=6$ after NAD with $\kappa=0.5$ and 30 iterations (third row), (c) sinc filter with $\alpha=12$ (first row), sinc filter with $\alpha=12$ after NAD with $\kappa=0.5$ and 5 iterations (second row), sinc filter with $\alpha=12$ after NAD with $\kappa=0.5$ and 30 iterations (third row).

This study was facilitated by the use of the BT platform. By exploring all possible values for the parameters describing the NAD filter, we were able for a short period to find the optimal values of the filter and to apply alone or in combination with other filters prior to the reconstruction resulting, namely in improved visualization of large low contrast breast features.

Nonlinear anisotropic diffusion filtering can improve image quality by removing noise while preserving details and even enhancing edges of the lesions which is critical for BT since its major advantage lies in the improvement of the detection and characterization of such lowcontrast masses.

This application reveals the usefulness and further developing and exploring of this GUI that allows access and exploitation of tomosynthesis algorithms for reconstruction of breast projection images obtained at different imaging conditions. 
Chapter 5

EVALUATION OF THE MPA ADAPTED FOR BREAST TOMOSYNTHESIS

WITH PARTIAL ISOCENTRIC ROTATION: A STRUCTURED PHANTOM

STUDY IN A CLINICAL SETTING

\section{CHAPTER SUMMARY}

In this chapter, the validity of using MPA adapted for BT with partial isocentric geometry, is investigated using real projection images acquired with phantoms at a clinical setting. The evaluation involved comparison of tomosynthesis images reconstructed with BP and 2D mammograms. The performance of the algorithms was evaluated, in combination with prefiltering of the projection images, in comparative studies that involved computation of several objective metrics. The following aspects were studied: the influence of the total phantom thickness and the position of the slab containing the objects of interest within the breast phantom, and the relationship between feature size and performance of reconstruction techniques. Results demonstrated that the MPA is a time efficient algorithm, fully compliant with and can be successfully used in BT clinical systems. Compared to 2D mammography, BT shows advantage in visualizing features of small size and for increased phantom thickness or features within a dense heterogeneous background with superimposed structures. 


\subsection{INTRODUCTION}

The MPA algorithm initially used for parallel and cone beam isocentric rotation, was further adapted for partial isocentric movement (Fig. 4.4) as described in previous section. In this case the detector is stationary while the source moves in a limited arc to acquire the projection images around the breast and this is the geometry adapted and used in most BT systems.

Aim of this work was to investigate the validity of the developed MPA algorithm using real projection images acquired with heterogeneous hardware breast phantoms at a clinical setting. The algorithm is evaluated in comparison to BP adapted for this geometry (Fig. 4.5).

For this purpose, a heterogeneous breast phantom of varying thickness was used with a Siemens Mammomat Inspiration System (Siemens AG, Germany). The performance of the two BT reconstruction algorithms was evaluated, in combination with pre-filtering of the projections using a median and a sinc filter suitably optimized for low- and high- contrast features, within a comparative study that involved computation of several objective metrics and visual assessment of reconstructed tomograms. To complete the study, a comparison between tomographic slices and two-dimensional (2D) mammograms was accomplished.

\subsection{EXPERIMENT}

\subsubsection{Breast phantom and image acquisition}

The phantom used was the CIRS-BR3D (CIRS Inc., Norfolk, VA), characterized by a heterogeneous background, mimicking a compressed breast. The phantom is composed of semicircular, equal-thickness slabs with dimensions of 100 x $180 \mathrm{~mm}$ and thickness of $10 \mathrm{~mm}$ each. The total phantom thickness was varied between $3 \mathrm{~cm}$ and $6 \mathrm{~cm}$.The slabs are made of two tissue equivalent plastic materials that are swirled together to create a heterogeneous structure with absorption characteristics corresponding to 50/50 mixture of glandular and adipose tissue. One of the slabs contains objects that simulate different breast lesions, such as masses, fibrils and $\mu \mathrm{Cs}$, as shown in Fig. 5.1. 


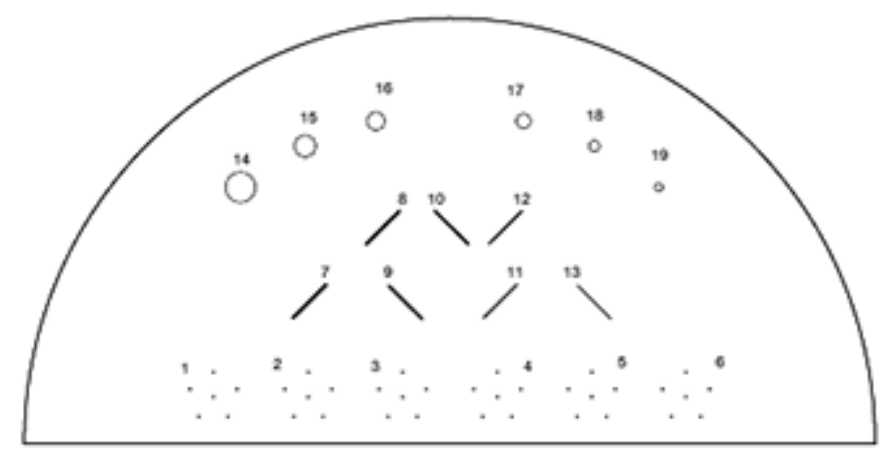

Fig. 5.1. Schematic representation of the CIRS-BR3D slab with the test objects.

Features 1-6 represent the 6 groups of $\mu \mathrm{Cs}\left(\mathrm{CaCO}_{3}\right.$ specks) with different grain sizes: 0.400, 0.290,0.230, 0.196, 0.165 and $0.130 \mathrm{~mm}$, while features 14-19 depict the six spheroid masses with the following diameters 6.32, 4.76, 3.96, 3.18, 2.38, and $1.80 \mathrm{~mm}$. The rest of the features (7-13) are fibers of $10 \mathrm{~mm}$ in length and diameters of $0.60,0.41,0.38,0.28,0.23$, 0.18 , and $0.15 \mathrm{~mm}$.

The acquisition protocol involved a combined measurement of a 2D mammogram followed by 25 tomosynthesis projections performed for each phantom configuration. A Siemens Mammomat Inspiration System (Siemens AG, Healthcare, Erlangen, Germany) with W/Rh anode/filter combination was used and the 25 projection images were acquired within an arc length of $50^{\circ}$. The system is equipped with an amorphous selenium detector $\left(239 \times 305 \mathrm{~mm}^{2}\right)$ with a pixel size of $85 \mu \mathrm{m}$. To acquire the images, the source was rotated around the phantom, while the detector remained in a stable position. Source to detector distance (SDD) was $650 \mathrm{~mm}$, while source to isocenter distance (SID) was $603 \mathrm{~mm}$. A schematic representation of the system and the breast phantoms as placed on the bucky are shown in Figs. 5.2(a) and 5.2(b). Measurements of five different phantom configurations were performed, obtained with different combinations of the CIRS BR3D slabs: one configuration, consisting of three slabs with a total thickness of $3 \mathrm{~cm}$ (Figs. 5.2(b)i); two phantoms composed of four slabs with a total thickness of $4 \mathrm{~cm}$ and different arrangement of the slice with the features of interest [Figs. 5.2(b)ii,iii] and two phantoms composed of six slabs with a total thickness of $6 \mathrm{~cm}$ and different arrangement of the slice with the features of interest were performed [Figs. 5.2(b)iv,v]. 


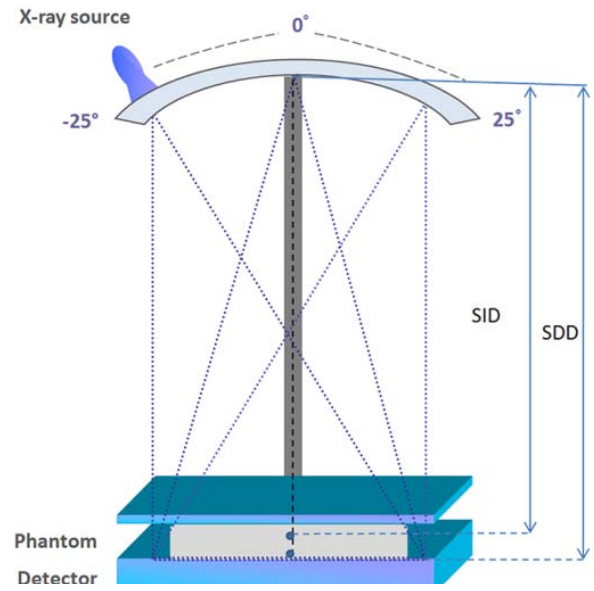

(a)

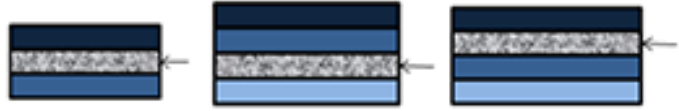

ii

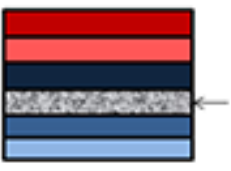

iv

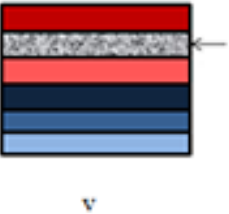

(b)

Fig. 5.2. A schematic representation of (a) the BT acquisition and (b) the arrangement of the slabs in the breast phantoms. The five different phantom arrangements for $3 \mathrm{~cm}, 4 \mathrm{~cm}$ and $6 \mathrm{~cm}$ are presented, with the arrow indicating the slice containing the features of interest: i) $3 \mathrm{~cm}$ and the slice of interest is placed in the middle at $1 \mathrm{~cm}$ above the imaging plate ii) $4 \mathrm{~cm}$ and the slice of interest is placed $1 \mathrm{~cm}$ above the imaging plate iii) $4 \mathrm{~cm}$ and the slice of interest is placed $2 \mathrm{~cm}$ above the imaging plate iv) $6 \mathrm{~cm}$ and the slice of interest is placed $2 \mathrm{~cm}$ above the imaging plate v) $6 \mathrm{~cm}$ and the slice of interest is placed $4 \mathrm{~cm}$ above the imaging plate.

The imaging modes and the exposure settings used for the acquisitions of the five phantom configurations at automatic exposure control are summarized in Table 5.1.

Table 5.1. Imaging acquisition details and calculated MGD for the five measurements performed in this study

\begin{tabular}{lllllllll}
\hline Set & $\begin{array}{l}\text { Phantom } \\
\text { Thickness } \\
(\mathbf{m m})\end{array}$ & $\begin{array}{l}\text { Compressed } \\
\text { Thickness } \\
\mathbf{( m m )}\end{array}$ & $\begin{array}{l}\text { Energy } \\
\mathbf{( k V p )}\end{array}$ & $\begin{array}{l}\text { HVL } \\
\mathbf{( W / R h},\end{array}$ & $\begin{array}{l}\text { ESAK } \\
\text { 2D } \\
\mathbf{( m G y )}\end{array}$ & $\begin{array}{l}\text { MGD } \\
\text { 2D } \\
\mathbf{( m G y )}\end{array}$ & $\begin{array}{l}\text { ESAK } \\
\text { BT } \\
\mathbf{( m G y )}\end{array}$ & $\begin{array}{l}\text { MGD } \\
\mathbf{\text { BT }} \\
\mathbf{( m G y )}\end{array}$ \\
\hline $\mathbf{i}$ & 30 & 25 & 27 & 0.525 & 1.06 & 0.54 & 2.11 & 1.07 \\
ii & 40 & 36 & 28 & 0.532 & 1.76 & 0.68 & 3.52 & 1.36 \\
iii & 40 & 36 & 28 & 0.532 & 1.79 & 0.69 & 3.58 & 1.39 \\
iv & 60 & 56 & 30 & 0.551 & 5.18 & 1.39 & 10.36 & 2.78 \\
$\mathbf{v}$ & 60 & 55 & 30 & 0.551 & 5.04 & 1.38 & 10.07 & 2.75 \\
\hline
\end{tabular}

The mean glandular dose (MGD) was estimated for each measurement, exploring the relationship between the MGD and the entrance surface air kerma (ESAK):

$$
M G D=E S A K g c s T
$$

In this expression, $g, c$ and $s$ are conversion parameters taken from Dance et al. (Dance et al 2011) depending on factors such as breast thickness, glandularity and half-value layer (HVL) of the incident spectra. The factor $T$ is used for BT acquisitions only and ranges from 0.98 for a 2cm breast to 0.964 for an $8 \mathrm{~cm}$ breast for the Siemens configuration. 


\subsubsection{Reconstruction methods and evaluation}

The projections were reconstructed in tomograms of $1 \mathrm{~mm}$ slice thickness using MPA adapted for the specific case of a stationary detector. The algorithm is presented in detail in section 4.3.1 and the position $r^{\prime}$ of each column on the "image formation plane" is given by Eq. 4.17.For comparison purposes, a BP algorithm was also developed for the acquisition geometry as described in section 4.3.2. The image reconstruction was performed on projection images, filtered prior to the reconstruction with median 3x3 filter and a sinc filters described by Eq. 4.1. An optimization study, presented thoroughly in Chapter 5, demonstrated that the use of these filters resulted in highest image quality of reconstructed high-contrast $\mu \mathrm{Cs}$ and low-contrast breast masses (Malliori et al 2012). Values of $\alpha$ in the range 1 to 4 and 8 to 12 were found optimal for high and low-contrast features, respectively. In this study a combination of median and sinc filters was used, with a value of $\alpha=2$ and $\alpha=12$ when $\mu C s$ and masses were reconstructed, respectively. The filtered projections were then reconstructed with both BP and MPA and are referred as (FBP) and (FMPA).

The features evaluated were six semi-spherical masses [features (12-17) in Fig. 5.1] and eighteen $\mu \mathrm{Cs}$ belonging to the three groups, with sizes between $400 \mu \mathrm{m}$ and $230 \mu \mathrm{m}$ [features (1-3) in Fig. 5.1].Each feature was evaluated using CNR and ASF. Additionally, to perform quantitative comparison between measurements accomplished with different dose levels, we used FOM (Eq. 2.4).

For the calculations, a circular ROI was considered inside each feature, while for the background, a 20 x $30 \mathrm{~mm}$ rectangular ROI was selected from the image in the area between mass 12 and group 1 of $\mu$ Cs (Fig. 5.1).

\subsection{RESULTS}

In total, twenty volumes were reconstructed by applying filtered and unfiltered MPA and BP. Twenty tomograms with features of interest and five 2D mammograms were processed. Results from the MPA were compared to the results obtained with BP. Detailed comparison and evaluation of these two algorithms with and without filtering was performed for breast masses and $\mu$ Cs present in the five phantoms. Filtered algorithms used values of 12 and 2 for $\alpha$ parameter when reconstructing breast masses and $\mu \mathrm{Cs}$, respectively. The evaluation also included comparison with the five 2D mammograms. 


\subsubsection{Comparison of MPA versus BP}

The same filtering method was used for both MPA and BP algorithms. In Fig. 5.3, ROIs that contain masses and $\mu$ Cs extracted from tomograms of breast phantoms with thickness of $3 \mathrm{~cm}$ (first column), 4cm (second column) and 6cm (third column) for both, the FMPA and FBP algorithms are shown.
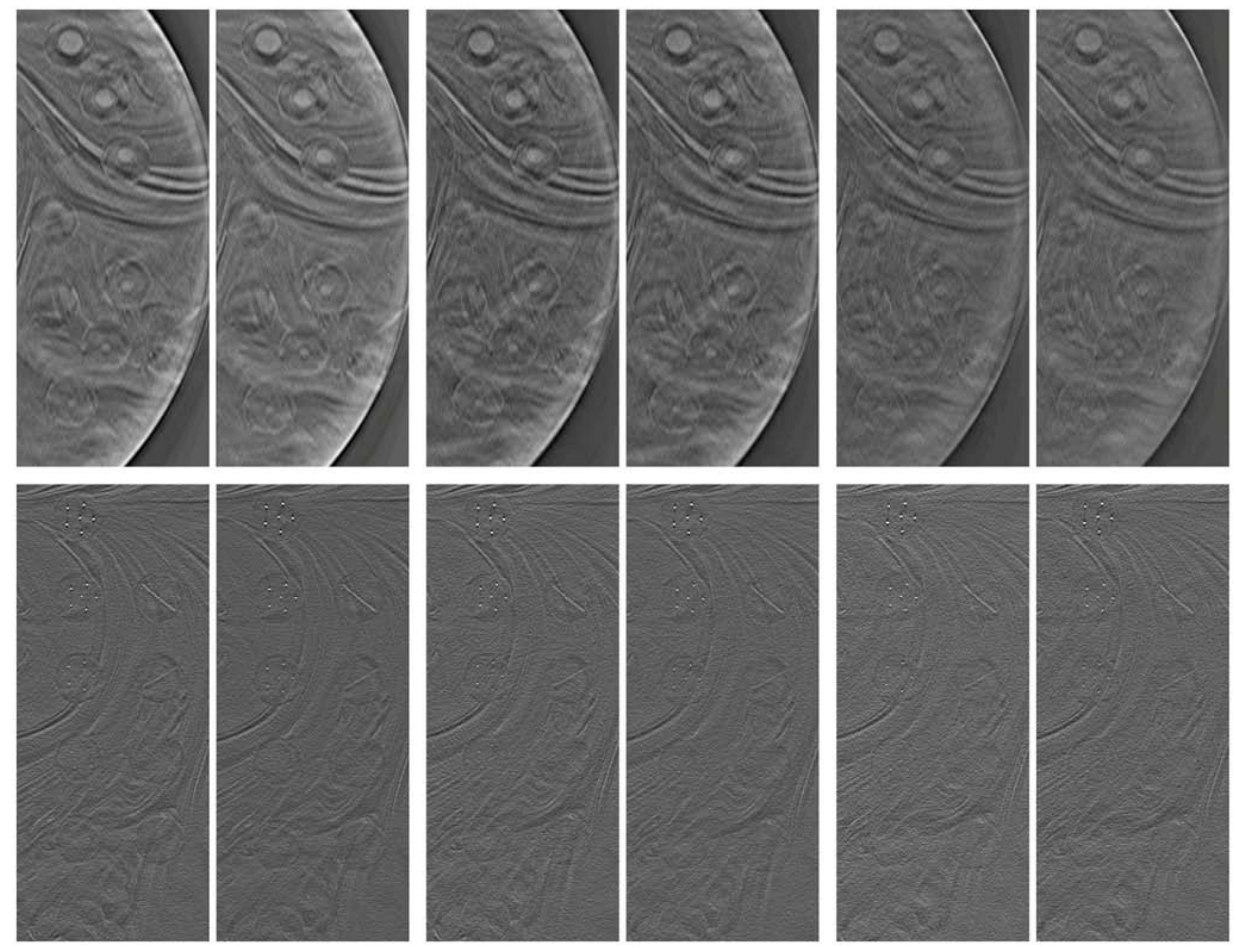

$3 \mathrm{~cm}$

$4 \mathrm{~cm}$

$6 \mathrm{~cm}$

Fig. 5.3. Comparison of tomograms that contain test objects like masses (first row) and $\mu$ Cs (second row) for different thicknesses: $3 \mathrm{~cm}$-set i (first column), $4 \mathrm{~cm}$-set ii (second column) and $6 \mathrm{~cm}$-set iv (third column) reconstructed with FMPA (left part in each column) and FBP (right part in each column).

Visual inspection between tomograms reconstructed with the two algorithms, showed no significant differences in feature detection or shape and size or the appearance of background tissue. This has been observed for all five breast phantoms. Both algorithms generated images with similar visual image quality, with the MPA however running much faster (about 20 times) in reconstruction process time. 


\subsubsection{Comparison of tomosynthesis slices with $2 \mathrm{D}$ projection mammography}

Tomographic in-focus slices reconstructed with simple and filtered MPA are shown in Fig. 5.4 in comparison to projection mammographic image, for the breast phantoms with thickness of $4 \mathrm{~cm}$ (set iii, from Table 5.1). Specifically, Fig. 5.4(a) depicts a tomography slice reconstructed with simple MPA, while Figs. 5.4(b) and 5.4(c) show tomograms reconstructed with FMPA with sinc filter parameters $\alpha=2$ and $\alpha=12$,respectively. The parameter value of $\alpha=12$ is used for breast masses, while $\alpha=2$ is used for the groups of $\mu$ Cs. The $2 \mathrm{D}$ projection image of the same breast phantom is shown in Fig. 5.4 (d). Both the filtered and unfiltered MPA can reconstruct the in-focus plane with sufficient quality. Most of the features of interest can be detected in all images including the 2D mammograms. However, it can be observed that high-contrast $\mu \mathrm{Cs}$ and low-contrast masses are best visualized with FMPA using $\alpha=2$ [Fig. 5.4(b)] and $\alpha=12$ [Fig. 5.4(c)], respectively.

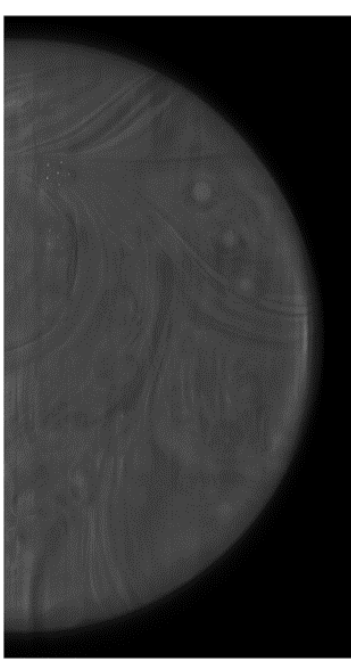

(a)

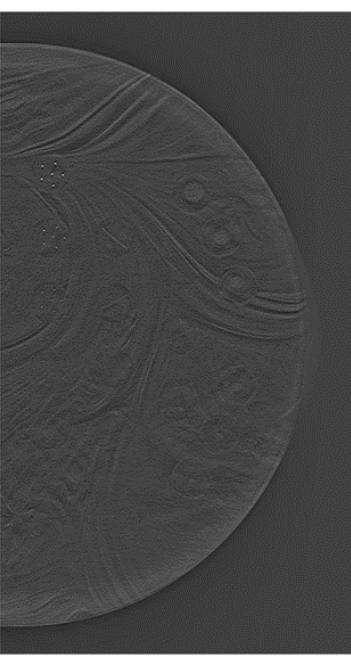

(b)

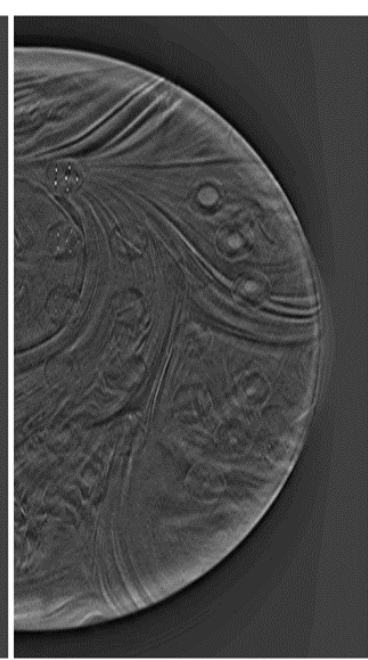

(c)

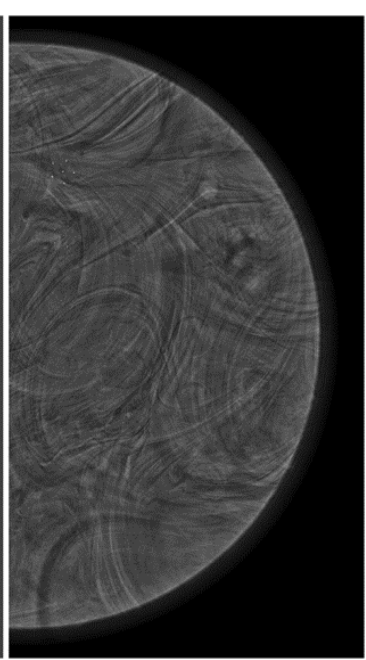

(d)

Fig. 5.4. Comparison of BT tomographic slices and 2D mammographic images obtained with a breast phantom of $4 \mathrm{~cm}$ thickness (set iii from Table 5.1): (a) simple (no filter) MPA, (b) FMPA ( $\alpha=2$ ), (c) FMPA (a=12) and (d) 2D projection mammography image.

Shown in Figs. 5.5 and 5.6 are ROIs taken from filtered tomographic images (FMPA) and 2D mammography projections that contain features of interest for all five breast phantom arrangements listed in Table 5.1. 


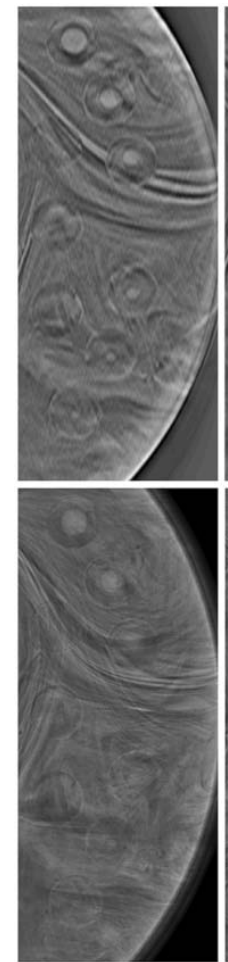

i
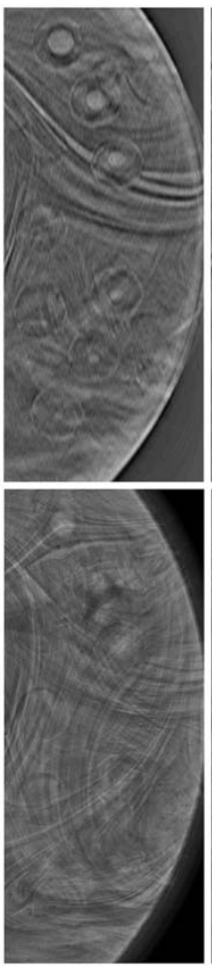

ii

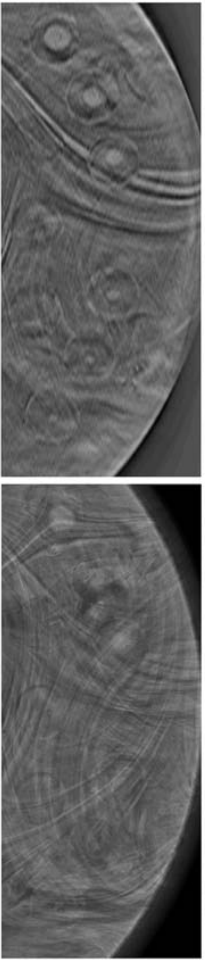

iii
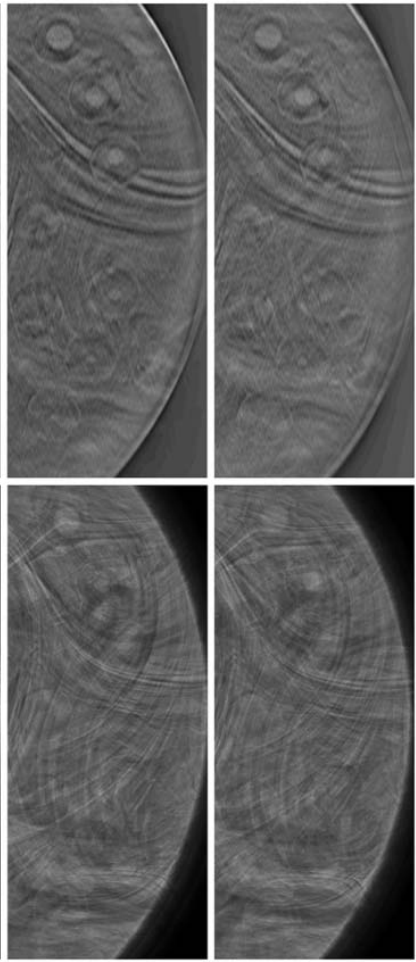

iv

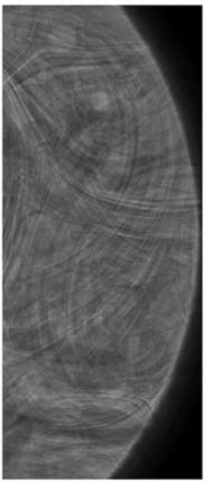

V

Fig. 5.5. ROIs showing the six low-contrast masses from FMPA $(\alpha=12)$ tomograms (upper row) and 2D images (lower row) for the different breast phantoms (i-v) from left to right.

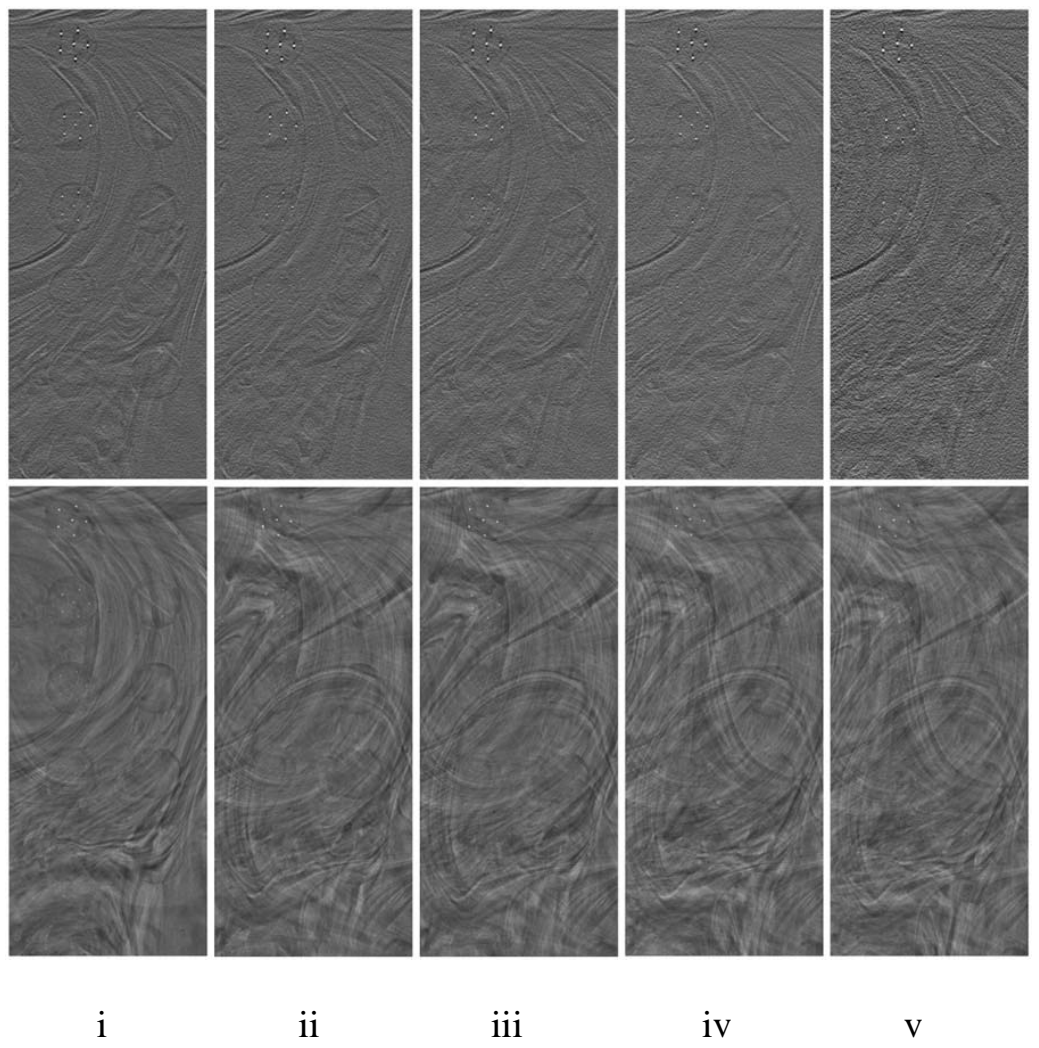

Fig. 5.6. ROIs showing the high-contrast groups of $\mu$ Cs and the fibers extracted from FMPA $(\alpha=2)$ tomograms (a) and 2D images (b) for the different breast phantoms (i-v). 
Increasing the breast thickness results in limitations of detection, especially for smaller masses, fibers and $\mu \mathrm{Cs}$. In all cases, the filtered tomograms allowed improved visualization of the features due to the removal of superimposed tissue compared to the 2D images. In the planar images, overlying structures may obscure futures of interest. This is demonstrated in the case of the breast mass with a size of $3.96 \mathrm{~mm}$ (feature 14), which is hardly detected in most of the 2D projection sets, while it is clearly visualized in all tomographic slices. The advantage for tomosynthesis is also evident for the three smaller masses with sizes in the range of 1.80 to $3.18 \mathrm{~mm}$ and the $\mu \mathrm{Cs}$ with sizes between 0.196 and $0.230 \mathrm{~mm}$ which can hardly be identified as such in the $2 \mathrm{D}$ images. Smaller $\mu$ Cs with sizes $0.165 \mathrm{~mm}$ and $1.130 \mathrm{~mm}$ could not be clearly perceived in any of both tomosynthesis and 2D mammography images.

Overall, FMPA reconstruction algorithm performs well on images obtained at clinical conditions.

\subsubsection{Evaluation of reconstruction algorithms as a function of phantom thickness}

\section{A. Low-contrast features performance}

Figures 5.7(a) and 5.7(b) depict the CNR and FOM calculated for the masses on both 2D and tomosynthesis images using filtered and non-filtered BP and MPA, for all five phantom configurations. The averaged values of CNR and FOM of all six masses in the in-focus slice are presented per phantom thickness. Figure 5.7(c) presents the ASF graph, for one of the masses with size of $6.32 \mathrm{~mm}$ and shows the performance of the algorithms for the lowcontrast feature in the $\mathrm{z}$ axis as we move away from the in-focus plane. The values are average ASF from all phantom sets.

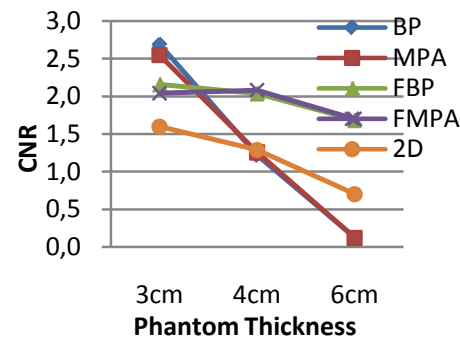

(a)

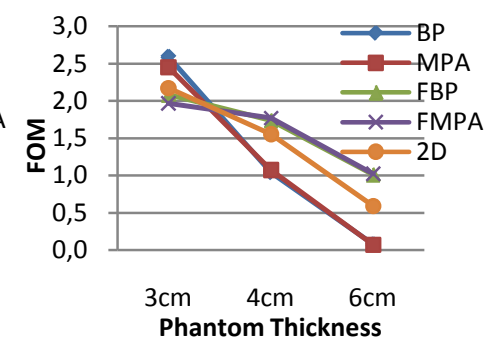

(b)

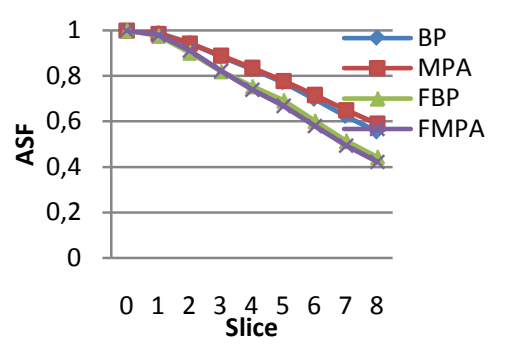

(c)

Fig. 5.7. Evaluation of algorithms reconstructing low-contrast features in terms of (a) CNR, (b) FOM and (c) ASF.

The quality of reconstructed masses with MPA and BP was similar. CNR differences between the two reconstruction techniques did not exceed 10\% for all masses. The filtered algorithms (FMPA and FBP) were also similar with an approximately constant CNR for all phantom 
thicknesses, while FOM was higher in case of thinner phantoms, indicating better CNR-todose performance.

Filtered algorithms outperform the non-filtered BP and MPA for the breast phantoms with thicknesses higher than $3 \mathrm{~cm}$. For the breast phantom of $3 \mathrm{~cm}$, the non-filtered BP and MPA showed better CNR and FOM [Fig 5.7(a) and 5.7(b)]. Moreover, the results for the CNR and FOM graphs outline the weak detection of the masses in the 2D images in the case of the $6 \mathrm{~cm}$ phantoms compared to the filtered tomograms. Overall, filtered BP and MPA had better performance compared to 2D for the case of masses, except for the $3 \mathrm{~cm}$ phantom, where the FOM value was higher for the 2D case. The latter, and generally the reduced difference between BT and 2D in terms of FOM values compared to CNR, can be explained taking also into consideration the lower doses used in the 2D mammography compared to BT during the combined 2D/3D acquisition (Table 5.1).

In the $\mathrm{z}$ axis, the ASF graphs [Fig. 5.7(c)] indicate better response for the filtered algorithms compared to non-filtered BP and MPA with a faster drop of the CNR as the distance from the in-focus plane is increased. The comparison between BP and MPA showed very similar data.

\section{B. High-contrast features performance}

Similarly to the case of masses, the groups of $\mu \mathrm{Cs}$ were evaluated for the 20 tomograms obtained with filtered and non-filtered BP and MPA and the 5 mammograms. For every image, $18 \mu \mathrm{Cs}$ have been evaluated and their values have been averaged for each phantom thickness separately. These $\mu \mathrm{Cs}$ belong to the three groups with sizes between $0.400 \mathrm{~mm}$ and $0.230 \mathrm{~mm}$. Calcifications with size smaller than $0.196 \mathrm{~mm}$ were not clearly detected in most of the images and therefore were not quantitatively evaluated. CNR and FOM metrics are presented as a function of the breast thickness in Figs 5.8(a) and 5.8(b) for the 2D images and the four tomosynthesis reconstruction techniques. Figure5.8(c) presents the ASF graphs for one group of $\mu \mathrm{Cs}(0.400 \mathrm{~mm})$ averaged for all the phantom configurations.

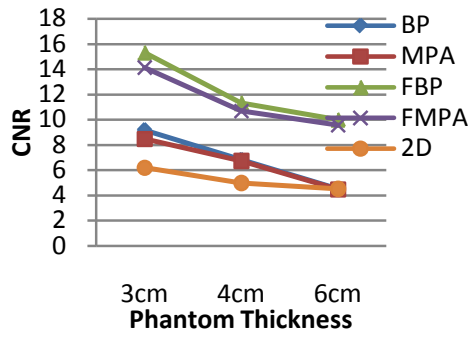

(a)

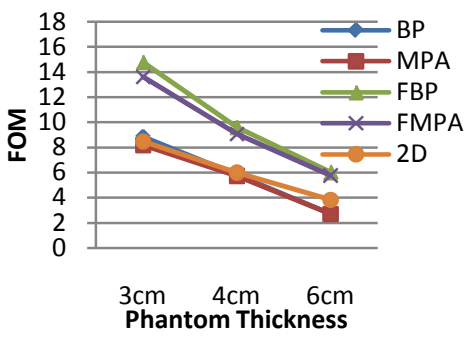

(b)

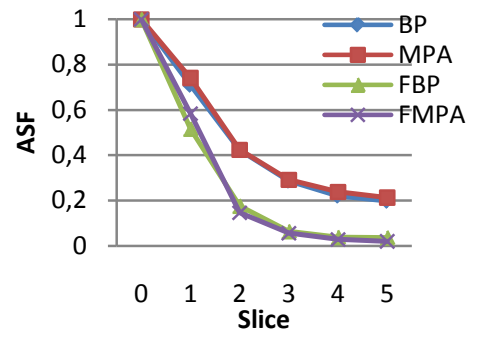

(c)

Fig. 5.8. Evaluation of algorithms reconstructing high-contrast features in terms of (a) CNR, (b) FOM and (c) ASF. 
The quality of test objects reconstructed with MPA and BP was similar with BP having slightly better CNR and FOM values compared to MPA. The same was observed between the filtered tomograms FMPA and FBP. None of the CNR differences between the MPA and BP reconstruction techniques exceeded $12 \%$ in the case of $\mu$ Cs reconstruction. Also, both FMPA and FBP had superior performance compared to2D images and non-filtered MPA and BP for all thicknesses in terms of all evaluation metrics as shown from the graphs in Figs. 5.8(a), 5.8(b) and 5.8(c). Specifically, the FOM values in case of filtered algorithms ranged between 1.6 and 2times higher for the filtered reconstruction techniques compared to the non-filtered BP and MPA and to the 2D mammograms for the different phantom thicknesses.

\subsubsection{Influence of the phantom configuration}

The influence on CNR and FOM, of the phantom configuration and of the position of slice containing the objects of interest (breast masses and $\mu \mathrm{Cs}$ ) within the breast phantom, was investigated for both MPA and FMPA tomosynthesis reconstruction algorithms. The comparison between CNR and FOM calculated for the different phantom configurations for the case of low-contrast and high-contrast features is shown in Figs.5.9 and 5.10, respectively. The average values of the six breast masses $(1.80 \mathrm{~mm}-6.32 \mathrm{~mm})$, in the former case (Fig. 5.9) and of the three groups of $\mu \mathrm{Cs}(0.230 \mathrm{~mm}-0.400 \mathrm{~mm})$, in the latter case (Fig. 5.10) were calculated for each phantom configuration. The figures summarize the evaluation that was performed on tomosynthesis images using MPA and FMPA and the results for the different phantom thicknesses are in agreement with the conclusions derived in the previous section.

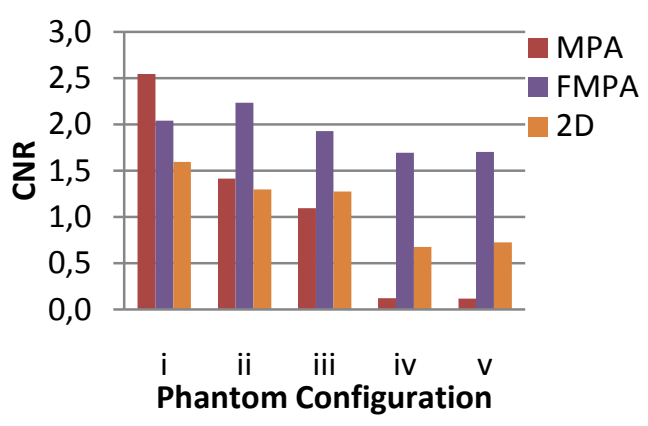

(a)

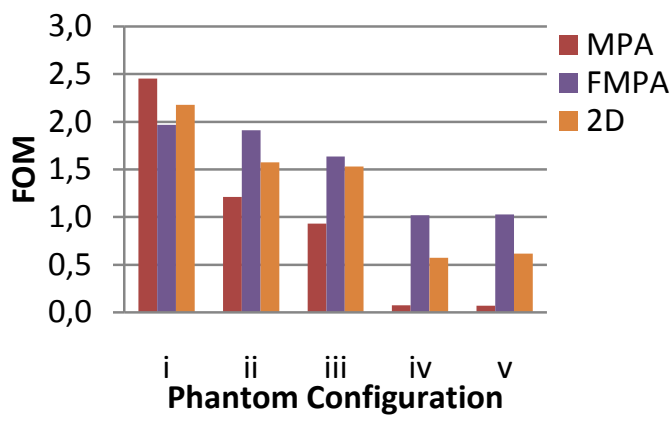

(b)

Fig. 5.9. Low-contrast features evaluation; (a) CNR and (b) FOM for the five different phantom configurations: i (3cm), ii and iii $(4 \mathrm{~cm})$, iv and v $(6 \mathrm{~cm})$. The evaluation has been performed for the 6 masses on the 2D images and the tomograms reconstructed with MPA and FMPA. 


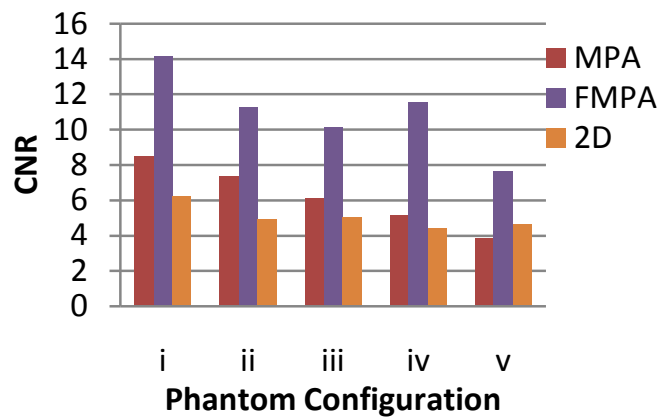

(a)

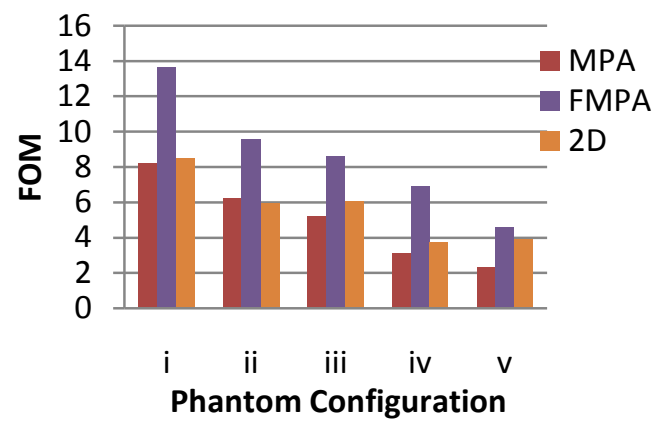

(b)

Fig. 5.10. High-contrast features evaluation; (a) CNR and (b) FOM for the five different phantom configurations: i $(3 \mathrm{~cm})$, ii and iii $(4 \mathrm{~cm})$, iv and $v(6 \mathrm{~cm})$. The evaluation has been performed for the 3 groups of $\mu \mathrm{Cs}(0.230 \mathrm{~mm}-0.400 \mathrm{~mm})$ on the $2 \mathrm{D}$ images and the tomograms reconstructed with MPA and FMPA.

In terms of CNR, FMPA outperformed 2D images in all phantom configurations for both masses and $\mu$ Cs [Figs. 5.9(a) and 5.10(a)]. Similarly, higher FOM values [Figs 5.9(b) and 5.10(b)] were observed in all cases except for the low-contrast features evaluation in the phantom of $3 \mathrm{~cm}$ thickness (set i). Overall, between the different phantom configurations best FOM results for the features evaluated were achieved for set $i$, characterized with a thickness of $3 \mathrm{~cm}$, while the worst were obtained for the $6 \mathrm{~cm}$ (set v).

Between the two different configurations for $4 \mathrm{~cm}$ (sets ii and iii) and $6 \mathrm{~cm}$ (sets iv and v) the 2D images have similar performance, while there seems to be an influence of the breast-like slabs arrangement for the tomosynthesis case. Specifically, for the high-contrast $\mu$ Cs slightly better FOM values were observed in both cases of $4 \mathrm{~cm}$ and $6 \mathrm{~cm}$ for the configurations where the slice with objects of interest was placed closer to the detector i.e. sets ii and iv, respectively [Fig. 5.10(b)].

\subsubsection{Influence of the object size}

\section{A. Low-contrast features performance}

Detailed comparison between filtered algorithms and 2D images is shown in Fig. 5.11 for three of the masses (features 14, 17 and 19) with different sizes $(6.32 \mathrm{~mm}, 3.18 \mathrm{~mm}$ and $1.80 \mathrm{~mm}$ ) representing a big, a medium and a small sized low-contrast mass. The results from the phantom sets of the same thickness i.e. ii,iii and iv, $\mathrm{v}$ were averaged. 


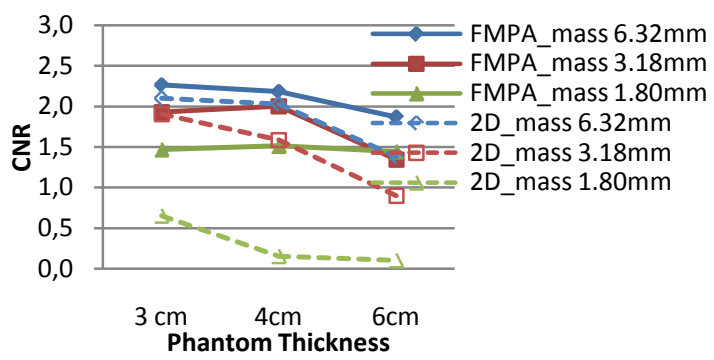

(a)

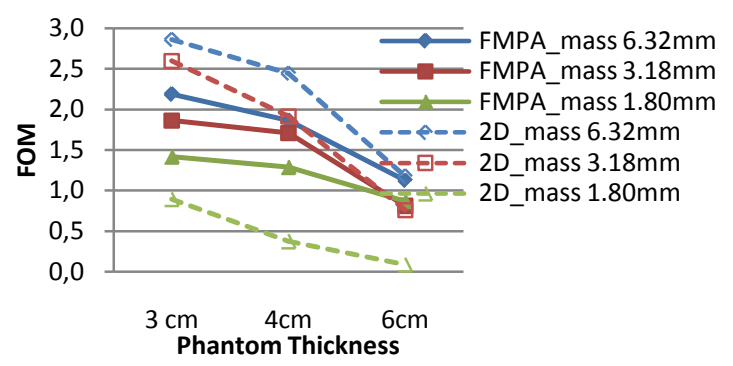

(b)

Fig. 5.11. Low-contrast features evaluation on both tomosynthesis reconstructed slices and 2D projection images for breast phantoms of 3 to $6 \mathrm{~cm}$ thick. The tomosynthesis slices were obtained with FMPA. Graphs presenting (a) CNR and (b) FOM for three of the masses with diameters of 1.80, 3.18 and $6.32 \mathrm{~mm}$.

In general, it is observed that the CNR and FOM values decrease as the size of the lowcontrast mass becomes smaller and the breast phantom thickness is increased in both 2D and BT images[Figs. 5.11(a) and 5.11(b)]. For the three masses and thicknesses investigated, CNR is improved in BT images compared to 2D demonstrating better visualization for these low-contrast features. The advantage seems to be higher with the decrease of the feature size and the increase of the phantom thickness. These results are in agreement with the visual observations in Fig. 5.5. The comparison of FOM values, which take also into account the lower doses used in 2D imaging, shows different trends between 2D and BT compared to the CNR values for these masses. Specifically, masses with sizes of 6.32 and $3.18 \mathrm{~mm}$ have higher FOM values on 2D images than on BT slices for breast thickness between 3 and $4 \mathrm{~cm}$ and a similar performance in case of a breast with a thickness of $6 \mathrm{~cm}$.In case of the $1.80 \mathrm{~mm}$ mass however, the improvement of FOM in BT is 1.6 to 5.2 times higher for the corresponding phantom thicknesses 3cm-6cm [(Fig. 5.11(b)] compared to 2D.

The conclusions derived, can change more in favor of BT, in case of masses that are totally or partially obscured by superimposed tissue as for example feature $16(3.96 \mathrm{~mm})$ which was discussed in section 4.5.3.B. Although this mass has a similar size as the medium mass evaluated in this section (feature 17), the FOMs in BT are higher compared to 2D. Specifically, the improvement is 1.6 and 5.4 times higher, for the sets of $\mathrm{i}(3 \mathrm{~cm})$ and $\mathrm{v}(6 \mathrm{~cm})$, respectively, where the mass is partially hidden by other structures and not clearly detected in 2D. Similarly, an improvement in FOM (4 times) was obtained for BT compared to 2D, in case of mass 15 , which is even larger in size $(4.76 \mathrm{~mm})$ for the set iv $(6 \mathrm{~cm})$.

Overall, apart from depth localization, BT shows advantages in visualizing low-contrast masses. This is more evident for small sized features at increased phantom thicknesses or for 
features within a highly dense heterogeneous background with superimposed anatomical structures.

\section{B. High-contrast features performance}

Detailed quantitative analysis of the different sized groups of $\mu \mathrm{Cs}(0.400 \mathrm{~mm}, 0.290 \mathrm{~mm}$ and $0.230 \mathrm{~mm}$ ) is presented in Fig. 5.12. The results from the phantom sets of the same thickness, i.e. ii,iii and iv,v were averaged, similarly to the case of the low-contrast features in the previous section, for simplicity purposes.

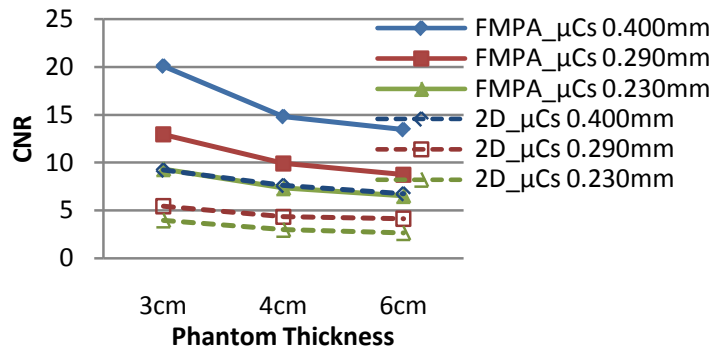

(a)

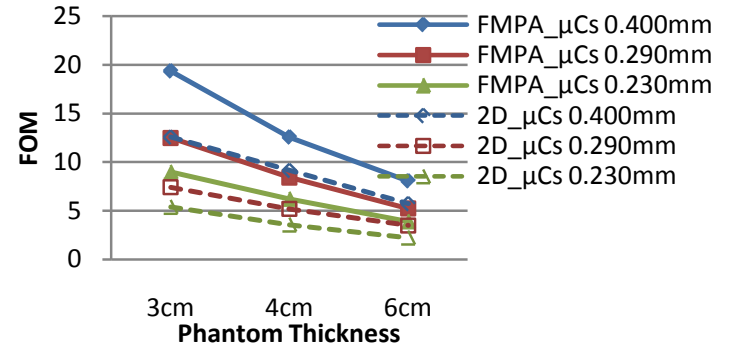

(b)

Fig. 5.12. Evaluation of the $2 \mathrm{D}$ images and the corresponding $\mathrm{BT}$ images reconstructed with FMPA for different phantom thicknesses (3cm-6cm) in terms of (a) CNR and (b) FOM for the three groups of $\mu$ Cs with sizes ranging from $0.230 \mathrm{~mm}$ to $0.400 \mathrm{~mm}$.

For all groups of $\mu \mathrm{Cs}$, FMPA resulted in higher CNR and FOM values compared to the 2D images, at each phantom thickness.

Similarly to the case of masses, the CNR and FOM values in BT and 2D images decrease as the $\mu$ Cs become smaller in size and the thickness of the phantom increases [Figs. 5.12(a) and 5.12(b)], confirming their visual appearance in images.

\subsection{CONCLUSIONS}

This study investigated the validity of the MPA digital tomosynthesis reconstruction algorithm adjusted for partially isocentric motion with stationary detector. The study showed that MPA reconstruction algorithm and developed filters perform well on images obtained with BT clinical system for both masses and $\mu \mathrm{Cs}$. The comparison between the MPA algorithm for stationary detector and a BP algorithm developed for the studied geometry showed close performance for the two algorithms with no considerable differences in feature detection, size and appearance of the background tissue, while at the same time the MPA allowed a multifold reduction in reconstruction time. 
Filtering the projection images prior to reconstruction, using optimized filters for each case of different low or high-contrast features, is important and results in images with better quality. The anatomical background was suppressed and the features especially those of small size, were much better visualized. The CNR values showed advantage of filtering for all $\mu$ Cs sizes and all phantom thicknesses. Similar results were obtained for the breast masses except for the $3 \mathrm{~cm}$ thick breast phantom. More specifically, the improvement was high for the $6 \mathrm{~cm}$ thick breast phantoms, where most of the low-contrast features were weakly detected without filtering prior to reconstruction. ASF graphs confirmed the advantage of filtering in the $\mathrm{z}$ axis as well, for all features. The study also revealed that features' location within phantoms of same thickness influenced slightly the quality of their reconstruction in tomosynthesis slices.

Compared to the 2D images of the five phantom configurations, FMPA tomograms showed their improved image quality, especially for low-contrast features as their size decreased and the phantom thickness increased or when surrounded by highly dense overlying tissue. Results from CNR comparison showed higher CNR values in BT for both masses and $\mu$ Cs in all breast phantom configurations. The FOM analysis, taking also into account the variation in dose levels between the sets of different thicknesses and acquisition modes, revealed a steady decrease in values as the phantom thickness increased, for both acquisition modes (2D/BT), and a clear advantage of FMPA reconstructed images over 2D images for the masses in the two thickest phantoms. For high-contrast $\mu \mathrm{Cs}$, FMPA had also higher FOM values compared to $2 \mathrm{D}$ for all phantom thicknesses and all the groups under evaluation. 


\section{ChAPTER 6}

\section{BREAST TOMOSYNTHESIS WITH MONOCHROMATIC BEAMS}

\section{CHAPTER SUMMARY}

Chapter 6 is dedicated to research activities involving monochromatic BT. Optimization of acquisition parameters and the performance of using BT with monochromatic irradiation has been studied thoroughly for the detection of low- and high-contrast breast lesions.

The investigations involved simulation and experimental work using simple software breast phantoms. Simulated images were formed using the XRayImagingSimulator while the reconstructions were performed with the BT platform. Detector and source characteristics as well as the acquisition geometry were taken into account based on the performed literature review and on a simulation study involving the testing of several combinations of acquisition arcs and number of projections. Experimental validation using monochromatic beams was performed at ELETTRA Synchrotron facilities. The first part, involved an extended optimization study in terms of acquisition geometry and filtering methods using a complex hardware phantom mimicking the breast.

The second part of the experimental work, involved acquisition of projection images with the objective to investigate potential benefits of using monochromatic beams compared to polychromatic, in image quality and features' visibility. This initial investigation with monochromatic and polychromatic 2D images, was extended for BT; a feasibility study was performed aiming to compare the performance of BT using monochromatic over polychromatic beams under the same acquisition conditions. The following simulation studies 
were carried out: (a) simulation of polyenergetic BT, (b) simulation of monochromatic BT and (c) mammography simulations with a simple (homogeneous) and a complex (heterogeneous) software breast phantom. A superior quality and lower dose for BT using monochromatic beams was reported.

\subsection{SIMULATION STUDIES: OPTIMISATION OF ACQUISITION PARAMETERS USING A HOMOGENEOUS PHANTOM}

\subsubsection{Introduction}

Despite the appearance of clinical BT systems and subsequent preliminary clinical studies using this technique, there is still much optimisation work that should be carried out for the improved use of these devices and choice of the most appropriate parameters, like acquisition geometries, x-ray source and detector characteristics, number of projection images, angular scan, algorithms for tomosynthesis reconstruction as well as blur removal due to source/detector and patient movement during acquisition. Optimization of acquisition arc and number of projection images per scanning arc for high quality reconstruction of breast images is a main research subject during the last five years. Initial simulation studies in this field were based on the design of homogeneous software phantoms approximating the x-ray attenuation properties of breast with embedded large low-contrast and small high-contrast breast-like lesions, such as masses and $\mu \mathrm{Cs}$ respectively. Studies with such phantoms have shown that in tomosynthesis extending the acquisition arc results in improved image quality of masses, except in the case that a simple back-projection method is used (Zhou et al 2007).

More complex simulation approaches used in optimization of the acquisition geometry concern simulation of three-dimensional (3D) structured backgrounds that may well approximate real 3D breast tissue distribution. One of the most extensive optimization studies, on the number of projection images and angular scan, was reported by Sechopoulos and Ghetti (Sechopoulos and Ghetti 2009). In this study, 63 acquisition geometries were simulated deriving from combinations of angular range and number of projection images. The projections were obtained from simulated breast tissue volumes with two features of interest that approximate a $6 \mathrm{~mm}$ soft tissue mass and a $0.4 \mathrm{~mm}$ microcalcification. This study reported that the extension of the angular range results in an increased reconstruction quality of masses. The study showed also that to maximize vertical resolution, the angular range needs to be extended, while at the same time there is an optimal number of projections per arc, not necessarily being the maximum number of projections. From all the combinations, the authors deduced that an acquisition arc of $60^{\circ}$ with 13 projections is the optimal 
combination for displaying low-contrast features, while the visualization of $\mu \mathrm{Cs}$ is not influenced by the angular range due to their inherent high contrast. Similar conclusions were derived from recent studies that investigated the detectability of breast lesions in tomosynthesis (Reiser and Nishikawa 2010, Van De Sompel et al 2011).

Aim of this study is to perform an investigation of acquisition parameters in BT using monochromatic beams. Synchrotron x-ray radiation, produced within the storage ring of the synchrotron facilities, is highly intense and monochromatic. However, due to the high cost of the experiments at these facilities and the limited time availability of the beam, simulation studies were performed prior to the experiments in place, at the given monochromatic x-ray energy. For this purpose, the BT platform was exploited to investigate the effect of the acquisition arc and the number of projections per arc on the quality of reconstructed features with varying contrast and size.

\subsubsection{Phantom and acquisition scenarios}

Initially, 37 acquisition geometries (10 acquisition arcs ranging from $16^{\circ}$ to $60^{\circ}$ and different number of projection imagesthat varied between 5 and 41) were tested using a homogeneous software phantom and MPA reconstruction algorithm. In the second phase, 16 acquisition sets were selected using four angular ranges $\left(3240^{\circ}, 48^{\circ}\right.$ and $\left.60^{\circ}\right)$ and four different angle intervals between projections per acquisition arcbased on higher CNR performance of the initial sets. The 16 combinations of the acquisition parameters are summarized in Table 6.1. The projection data generated from these acquisition geometries were used in a BT study involving reconstruction with MPA without and with pre-filtering of the projections (S-MPA, F-MPA), evaluation of image quality in terms of CNR and C for low- and high-contrast features and classification of the acquisition arcs and selection of optimal number of projections for the different type of features. The ROIs of the tomosynthesis slices are presented along with the quantitative results in this section.

The projection images were obtained from a simulated version of the CIRS 011A breast phantom (manufactured by Computerized Imaging Reference Systems, Inc., Norfolk, VA) with a homogenous background (Fig. 6.1) designed with the phantom modelling module of the X-Ray Imaging Simulator (Bliznakova 2003).

The ROIs containing low- and high-contrast features that were used for the evaluation along with their sizes are shown in Fig. 6.1. 


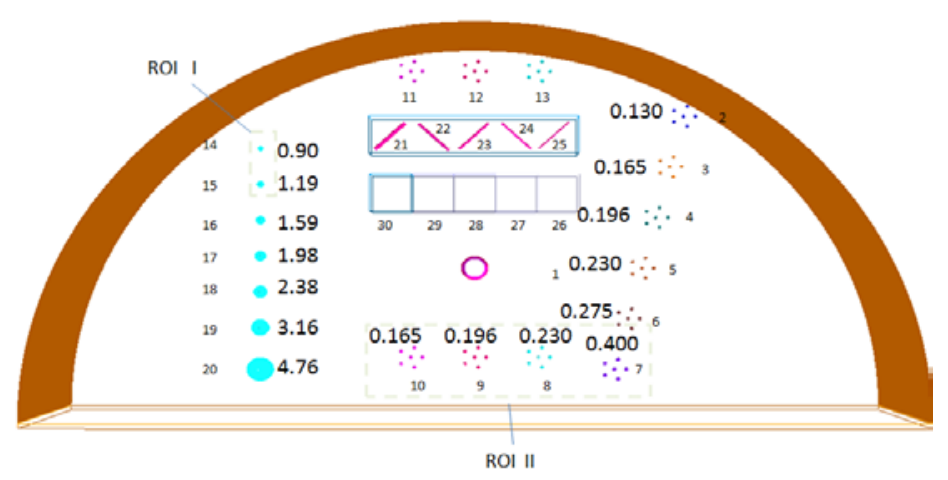

Fig. 6.1. CIRS Breast phantom - software design.

However, in this case poisson statistics was added to simulate photon noise, making images more realistic and closer to clinical conditions. The incident number of photons was calculated according to the incident air kerma (Dance et al 2000a).The mean glandular dose in all acquisition sets was equal to 2.5mGy that corresponds to the dose used in conventional mammography for breasts with an average thickness of $4.5 \mathrm{~cm}$ (Perry et al 2006). Projection images were simulated also with the XRayImagingSimulator. These images are twodimensional distributions of the number of photons reaching the detector.

Table 6.1. Acquisition schemes

\begin{tabular}{cll}
\hline Angular range & Number of & Angle inc. \\
& Projections & $2^{\circ}$ \\
\hline $60^{\circ}$ & 31 & $3^{\circ}$ \\
& 21 & $4^{\circ}$ \\
16 & $6^{\circ}$ \\
& 11 & $2^{\circ}$ \\
& 25 & $3^{\circ}$ \\
& 17 & $4^{\circ}$ \\
& 13 & $6^{\circ}$ \\
& 9 & $1^{\circ}$ \\
& 41 & $2^{\circ}$ \\
& 21 & $4^{\circ}$ \\
& 11 & $5^{\circ}$ \\
& 9 & $1^{\circ}$ \\
& 33 & $2^{\circ}$ \\
& 17 & $4^{\circ}$ \\
& 9 & $8^{\circ}$ \\
\hline
\end{tabular}


The volumes were reconstructed using the computer-based platform developed for tomosynthesis studies (Malliori et al 2010). In the case of FMPA, a sinc filter was applied prior to the reconstruction (see Eq. 4.1). The filter's parameter $\alpha$ was adjusted to perform well in both cases of high- and low-contrast features. For the high-contrast Cs, the filter's parameter $\alpha$ was set to 0.1 . Preliminary work with varying values of $\alpha$ showed that, lowcontrast masses are very well visualized for $\alpha=12$ (Malliori et al 2012).

\subsubsection{Results}

\section{A. Comparison of SMPA and FMPA}

Figures 6.2 and 6.3 show low- and high-contrast features reconstructed with SMPA and FMPA.

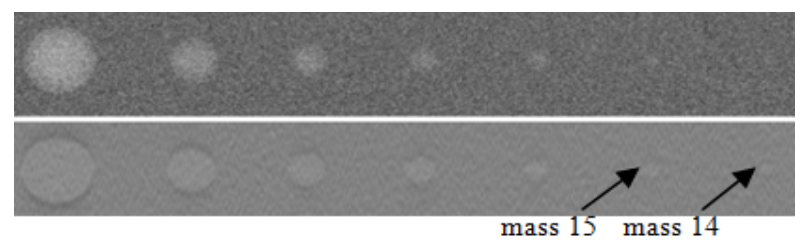

Fig. 6.2. Low-contrast features reconstructed with SMPA (upper row) and FMPA (lower row).

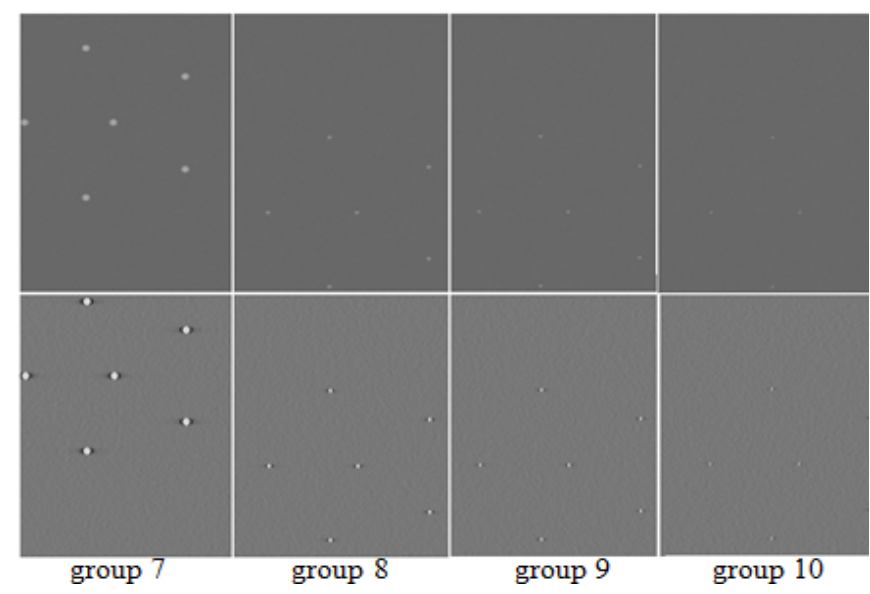

Fig. 6.3. High-contrast features (groups 7-10) reconstructed with SMPA (upper row) and FMPA (lower row).

In figure 6.2, the noise in the volume reconstructed with SMPA reduces the image quality. The edges of the features are not clearly defined while the smallest mass (feature 14, 0.90mm) is not identified at all. The quality of tomograms reconstructed with FMPA is much improved. All masses are well visualized; the edges are sharper while the noise is suppressed.

The observations for the low-contrast features in Fig.6.2 stand for the high-contrast case too (Fig. 6.3). The tomograms reconstructed with S-MPA have poor contrast and the $\mu \mathrm{Cs}$ with the 
smallest size (group 10) are hardly identified whereas, tomograms reconstructed with FMPA have better quality and all groups of Cs are well visualized.

The superiority of FMPA is also quantitatively supported by the CNR and C evaluation shown in Fig. 6.4. The results presented are the mean values for the 16 acquisition schemes. For the low-contrast features, the CNR and $\mathrm{C}$ values for the smallest mass identified with both algorithms are depicted (mass 15). Similarly, for the high-contrast features, the CNR and C values of group 9 of Cs are averaged and presented.
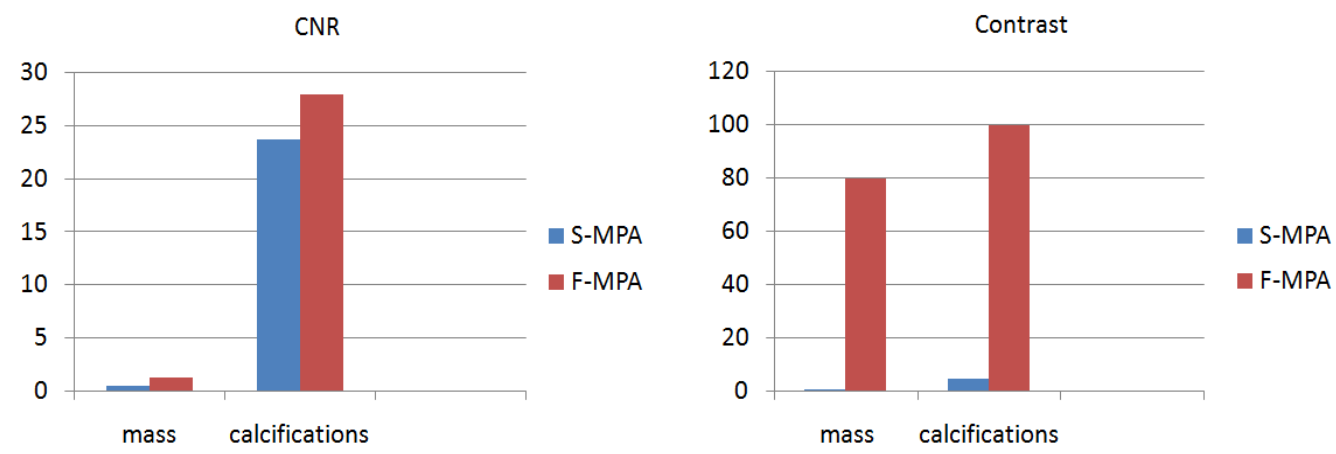

Fig. 6.4. CNR and C values for SMPA and FMPA. The results shown are the mean values for the 16 acquisition schemes. For the low-contrast features, mass 15 (size $1.19 \mathrm{~mm}$ ) is evaluated. For the highcontrast features, Cs belonging to group 9 (size $0.196 \mathrm{~mm}$ ) are averaged and evaluated.

The results indicate an improved performance for the FMPA. Contrast values in particular, are significantly higher for FMPA in all cases compared to SMPA.

Similar trends in the graphs were observed for the rest low- and high-contrast features of bigger size.

B. Image quality of reconstructed features with the arc length for different number of projections

Tables 6.2 and 6.3 summarize the optimal number of projections for each acquisition arc for features with different contrast and size. The results are classified in descending order according to their CNR values. All results presented in the tables are for volumes reconstructed with FMPA which demonstrated better performance for both low- and highcontrast features as described in the previous section. 
Table 6.2. Optimal acquisition geometry for mass $15(1.19 \mathrm{~mm})$ in descending CNR order

\begin{tabular}{lccc}
\hline \multicolumn{1}{c}{ Acquisition geometry } & Angle inc. & CNR & C \\
\hline $60^{\circ}, 11$ projections & $6^{\circ}$ & 1.69 & 85.83 \\
$48^{\circ}, 13$ projections & $4^{\circ}$ & 1.56 & 84.35 \\
$40^{\circ}, 21$ projections & $2^{\circ}$ & 1.38 & 79.52 \\
$32^{\circ}, 9$ projections & $4^{\circ}$ & 1.25 & 81.18 \\
\hline
\end{tabular}

Table 6.3. Optimal acquisition geometry in descending CNR order for each group of Cs

\begin{tabular}{clcl}
\hline Size of Cs & Acquisition geometry & Angle inc. & CNR \\
\hline $0.400 \mathrm{~mm}$ & $32^{\circ}, 17$ projections & $2^{\circ}$ & 30.13 \\
(Group 7) & $40^{\circ}, 21$ projections & $2^{\circ}$ & 29.57 \\
& $48^{\circ}, 25$ projections & $2^{\circ}$ & 28.65 \\
& $60^{\circ}, 31$ projections & $2^{\circ}$ & 27.50 \\
$0.230 \mathrm{~mm}$ & $32^{\circ}, 9$ projections & $4^{\circ}$ & 24.34 \\
(Group 8) & $40^{\circ}, 11$ projections & $4^{\circ}$ & 23.00 \\
& $48^{\circ}, 13$ projections & $4^{\circ}$ & 22.90 \\
& $60^{\circ}, 11$ projections & $6^{\circ}$ & 21.56 \\
$0.196 \mathrm{~mm}$ & $40^{\circ}, 9$ projections & $5^{\circ}$ & 31.46 \\
(Group 9) & $32^{\circ}, 9$ projections & $4^{\circ}$ & 31.43 \\
& $48^{\circ}, 13$ projections & $4^{\circ}$ & 28.61 \\
& $60^{\circ}, 11$ projections & $6^{\circ}$ & 27.12 \\
& $32^{\circ}, 5$ projections & $8^{\circ}$ & 27.26 \\
$0.165 \mathrm{~mm}$ & $40^{\circ}, 9$ projections & $5^{\circ}$ & 26.13 \\
(Group 10) & $48^{\circ}, 9$ projections & $6^{\circ}$ & 24.16 \\
& $60^{\circ}, 11$ projections & $6^{\circ}$ & 21.75 \\
\hline
\end{tabular}

All features from the two ROIs that were tested were successfully identified using F-MPA with a sinc filter suitably adjusted for each case of high- and low-contrast features. In detail, F-MPA indicated better performance in terms of noise reduction and superior edge visualization for low- and for high-contrast features especially for the ones with the smallest dimensions that were not identified at all without filtering. Quantitative analysis, confirmed the observations; F-MPA reconstruction improved the CNR values and resulted in images with significantly higher contrast compared to S-MPA. 
The CNR classification for the studied mass (Table 6.2) revealed that CNR tends to increase with extending the arc length. Best results were achieved for the $60^{\circ}$ acquisition arc. An opposite behavior was observed for the high-contrast features (Table 6.3).

The evaluation for the different acquisition schemes indicated that the number of projections for each arc length is as important as optimizing the acquisition arc. In case of Cs, where different feature sizes were evaluated, the optimal step increment that defines the number of projections per arc tends to increase as the size of feature to be identified decreases. For Cs with size of $0.4 \mathrm{~mm}$, the optimal acquisition sets were obtained with å Znterval between projections for each angular range. For smaller sizes between $0.196 \mathrm{~mm}$ and $0.230 \mathrm{~mm}$ the dominant angle interval was $4^{\circ}$ while this value increased even more for the smallest group that was tested $(0.165 \mathrm{~mm})$. This can be explained due to the fact that increasing the step and thus reducing the number of views leads to higher dose per projection which enables such small sized high-contrast features to be detected and better visualized in a homogeneous background. However, in the general case it was found that maximizing or minimizing the number of projections in each arc, does not necessarily bring the same result on CNR and the optimal performance was achieved for angle intervals in between which is in agreement with the study of Sechopoulos and Ghetti (Sechopoulos and Ghetti 2009).

\subsection{EXPERIMENTAL WORK AT ELLETRA SYNCHROTRON FACILITIES: OPTIMISATION OF ACQUISITION PARAMETERS USING A HETEROGENEOUS PHANTOM}

\subsubsection{Introduction}

The previous section was dedicated to optimization of the acquisition parameters performed with simulations. However, these findings should be verified at clinical systems. Ren et al. (Ren et al 2006) studied experimentally the effect of 11, 15 and 21 number of projections on CNR for a given dose and $20^{\circ}$ angular scan. For the case of $6 \mathrm{~mm}$ mass they showed that as the number of projections increases, the maximal achievable CNR decreases. Zhao et al. (Zhao et al 2009) showed an improved mass visibility when the angular range is increased. In addition, $\mu \mathrm{Cs}$ of medium size ( $240 \mu \mathrm{m}$ to $540 \mu \mathrm{m}$ ) were detectable with the acquisition arcs tested, $20^{\circ}$ and $40^{\circ}$. However, a study including $\mu \mathrm{Cs}$ with smaller sizes has not been performed yet in order to identify the optimal angular range in their detection. These experimental studies were also limited to the use of homogeneous mammographic phantoms.

Experimental studies with complex breast phantoms have been carried out to compare visibility of features in tomosynthesis against full field digital mammography (FFDM). In a 
preliminary study, Smith et al. (Smith et al 2006) showed that the visibility of low-contrast objects using tomosynthesis was superior compared to the visibility of these objects in FFDM, even when tomosynthesis was performed with less dose. Tomosynthesis also showed superior visibility over FFDM for $160 \mu \mathrm{m}$ to $180 \mu \mathrm{m} \mu \mathrm{Cs}$. In a similar experimental investigation, Bertolini et al. (Bertolini et al 2011) reported that the high contrast details with size smaller than $1 \mathrm{~mm}$ had higher CNR values on BT slices compared to FFDM. This is still to be investigated, since previously Timberg et al. (Timberg et al 2010) found no advantage of BT in the detection of $\mu \mathrm{Cs}$ with size smaller than $200 \mu \mathrm{m}$. In addition, an extensive experimental study on the impact of the acquisition arc on the image quality of reconstructed tomosynthesis slices is still to be conducted.

Aim of the experiments in this phase was to validate the results of the simulation study and to further perform an optimization study for this geometry involving evaluation of filtering methods using a complex phantom. This section presents the results from our study on the impact of the acquisition arc on the image quality of small high-contrast features, denoted as $\mu \mathrm{Cs}$, with size ranging from $170 \mu \mathrm{m}$ to $500 \mu \mathrm{m}$ and a $6 \mathrm{~mm}$ low-contrast mass, embedded within a highly heterogeneous background. These features mimic various breast lesions. Images were obtained at the SYRMEP beamline at ELETTRA Synchrotron Light Laboratory, Trieste, Italy. The basic reconstruction algorithm was the MPA. Filters were used along with the MPA to produce filtered tomosynthesis slices. Initially, an optimization study was carried out to set the parameters of the filter for the cases of presently studied features. The quality of the reconstructed features was assessed visually and quantitatively.

\subsubsection{Experimental Setup and methods}

\section{A. Phantom arrangement}

The phantom used for the experiments is an ensemble composed of the $1.5 \mathrm{~cm}$ TORMAM and the $1 \mathrm{~cm}$ TORMAX mammography test phantoms. The TORMAX phantom contains low and high-contrast features surrounded by a homogeneous background. The TORMAM phantom consists of two parts: the left half contains a range of filaments, microparticles and low-contrast details, representing pathological features in the breast, while the right half consists of materials that simulate the appearance of breast tissue with embedded groups of $\mu \mathrm{Cs}$. These two phantoms were stacked together, with the TORMAX placed in front of the TORMAM, composing a phantom with a highly heterogeneous background and a thickness of $2.5 \mathrm{~cm}$. Two pieces of polymethymethacrylate (PMMA) test blocks were placed supplementally on both sides as shown in Fig.6.5, increasing thus the total thickness of the 
phantom arrangement to $4 \mathrm{~cm}$. This composite phantom, named MAMMAX, has a realistic shape and size and approximates a breast of $4.5 \mathrm{~cm}$ equivalent thickness composed of $50 \%$ adipose and $50 \%$ glandular tissue.

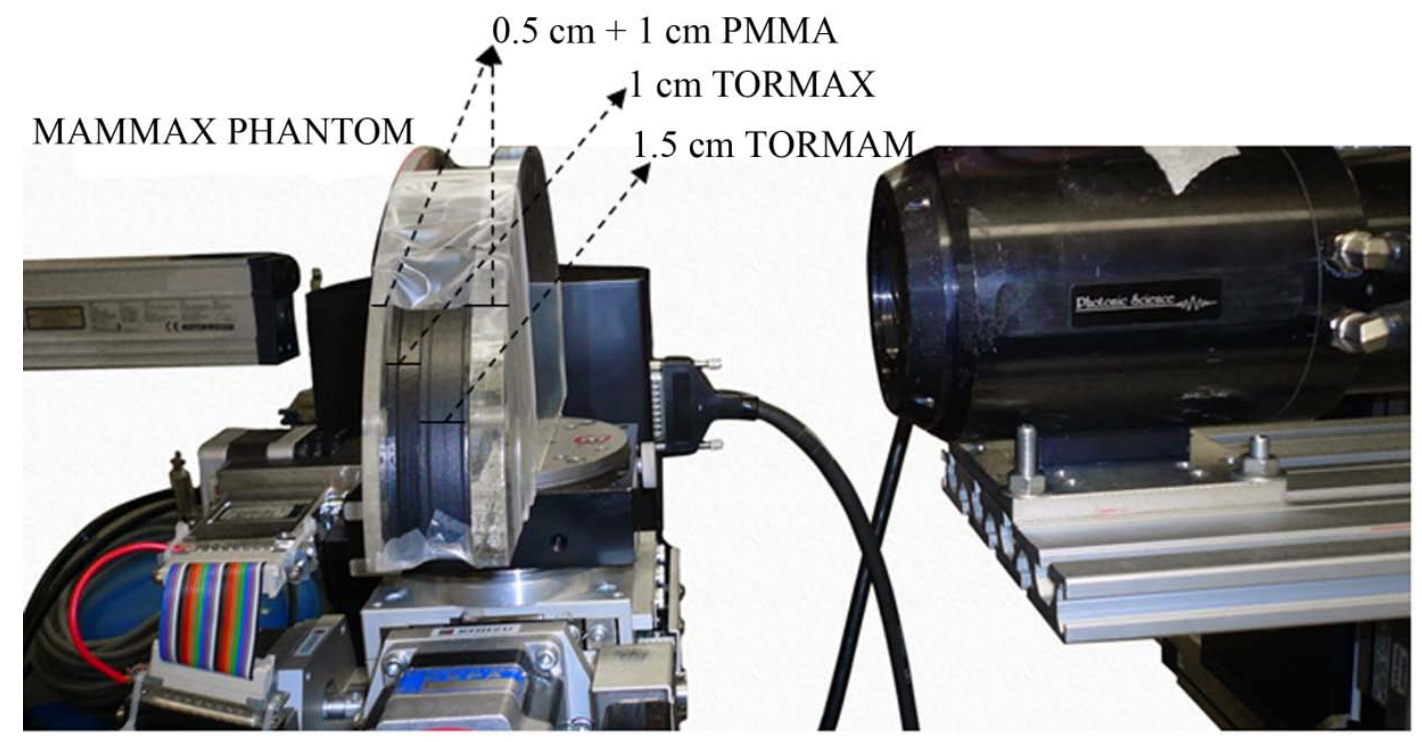

Fig. 6.5. Photograph of the experimental setup at SYRMEP beamline. The designed phantom (MAMMAX) placed on the sample rotator between the X-ray source and the detector.

\section{B. Image acquisition}

The experiments were performed, as discussed also in Chapter 3, at the SYRMEP beamline, designed for research in medical diagnostic radiology at ELETTRA, Trieste. The optics are based on a double-crystal $\mathrm{Si}$ (111) monochromator which works in an energy range between 8 $\mathrm{keV}$ and $35 \mathrm{keV}$, while the photon beam is slit and its position is fixed in space. At a distance of about $20 \mathrm{~m}$ from the source, the area of the x-ray beam was $98 \times 2.5 \mathrm{~mm}^{2}$. In order to obtain two dimensional projection images, the phantom and the detector were scanned vertically, with a scan speed of $2 \mathrm{~mm} / \mathrm{s}$.

For the acquisition of the two-dimensional projection images of the composite phantom, the latter was placed on top of a rotating holder in front of the monochromatic source at a distance of $16.5 \mathrm{~cm}$ from the detector (Fig. 6.5). Images at different acquisition arcs were obtained with the phantom stage rotating around an axis that was perpendicular to the incident beam at the desired angles.

The detector was a water cooled, charge coupled device camera (Photonic Science x-ray Hystar, $2048 \times 2048$ full frame, 16 bit, $56 \mu$ m pixel -obtained after a $4 \times 4$ pixel binning-, $28.67 \times 28.67 \mathrm{~mm}$ field of view). Cooling detector was used to keep electronic noise in 
relatively low levels. To further reduce the noise impact, which was dominated by the 'dark current noise', all images were corrected by subtracting an average image calculated from five dark-current images obtained immediately after the acquisition of phantom images. Images were then normalized to a flat-field image acquired at the same energy. During experimentation, the incident exposure was being monitored with an ionisation chamber placed in front of the sample. The readings of the chamber were then converted to photon flux incident on the phantom by an in-house computer program at the SYRMEP (Bovi et al 2007).

The size of all images was 512 pixels in both directions. The region being imaged was the lower right part of the MAMMAX phantom. Specifically, the part belonging to TORMAX consists of $6 \mathrm{~mm}$ low-contrast circular details and 0.25 and $0.5 \mathrm{~mm}$ high-contrast details embedded into a homogeneous background. These features are depicted in Fig. 6.6(a), which presents the single projection image obtained from imaging the TORMAX phantom alone. Schematically, the features located in the imaged region from the TORMAX are shown in Fig. 6.6(b). For visualization purposes, the background has been removed, outlining the position and dimensions of the features, with the darker ones having higher attenuation coefficients. The part belonging to TORMAM, on the other hand, contains $\mu \mathrm{Cs}$ in addition to fibrous and nodular details. This heterogeneous background provides a more "natural” image of the breast. Figure 6.6(c) shows a projection image of the whole composite phantom MAMMAX, taken at $17 \mathrm{keV}$ with the beam being perpendicular to the phantom central plane (as in the arrangement shown in Fig. 6.5). In Fig. 6.6(d), the background of the combined MAMMAX phantom has been removed and the two types of features under evaluation are displayed: (a) the low-contrast circular detail (a mass of $6 \mathrm{~mm}$ ) that belongs to the TORMAX phantom and (b) several $\mu \mathrm{Cs}$ from the two groups $\mathrm{A}$ and $\mathrm{B}$ that belong to the TORMAM phantom. The size of the four features from these groups denoted with a-d ranged from 250 to $500 \mu \mathrm{m}$, while the size of the rest denoted with e-l ranged from 170 to $220 \mu \mathrm{m}$. All the studied features from both groups are high-contrast features of small dimensions. However, since a poor performance of $\mu \mathrm{Cs}$ with size smaller than $200 \mu \mathrm{m}$ has been previously reported in the case of BT, (Timberg et al 2010) the $\mu \mathrm{Cs}$ have been further grouped in the above size categories and studied separately. Calcifications with size smaller than $170 \mu \mathrm{m}$ were not studied since they were difficult to distinguish from artifacts in the background. 


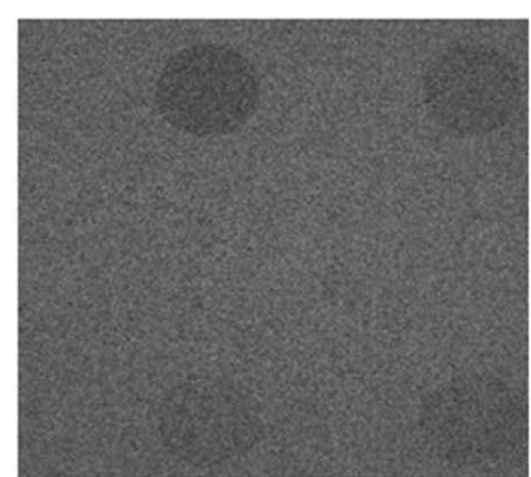

(a)

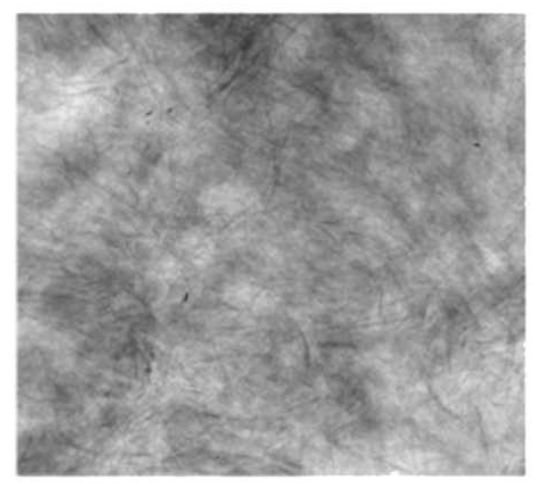

(c)

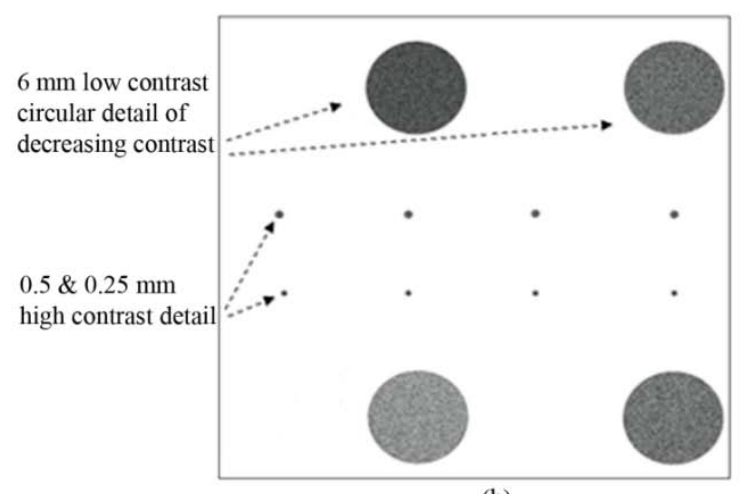

(b)

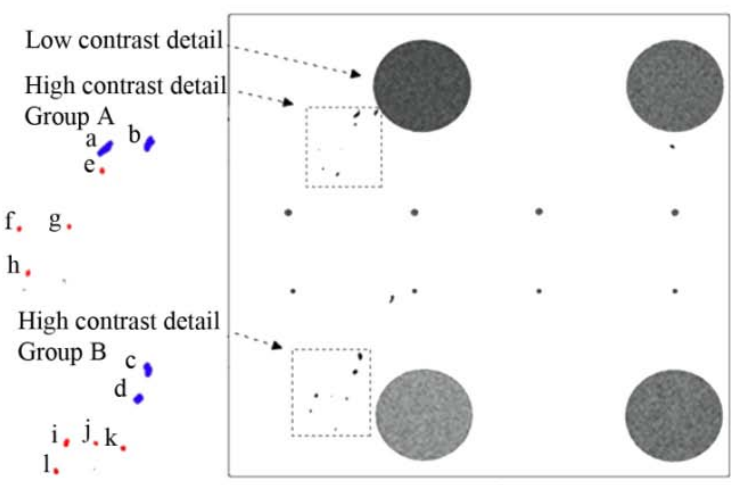

(d)

Fig. 6.6. ROI of (a) projection images from TORMAX phantom alone (b) features of interest in TORMAX that are projected without the homogeneous background, (c) projection image from the MAMMAX phantom (d) features of interest in the MAMMAX phantom with the heterogeneous background removed.

\section{Acquisition Scenarios}

The following images from the MAMMAX breast phantom were experimentally obtained: (a) a single projection image of the ROI with an incident beam energy of $17 \mathrm{keV}$; (b) a limited number of projection images in tomosynthesis acquisition arcs of $24^{\circ}, 32^{\circ}, 40^{\circ}, 48^{\circ}$ and $56^{\circ}$ at an incident energy of $17 \mathrm{keV}$.

The projection images for tomosynthesis were acquired using a constant angle interval; i.e. every $4^{\circ}$ in the corresponding arc. This value enabled a limited number of projections per arc and was selected taking into consideration the former simulation studies, literature review and the attempt to reduce acquisition time in Synchrotron facilities. In all these cases, the MGD to the breast phantom was set to $2.5 \mathrm{mGy}$ according to the European guidelines for quality assurance in breast cancer screening and diagnosis for a standard one side mammography (Perry et al 2006). The incident air kerma (K) measured at the upper surface of the breast phantom, was calculated for all tomosynthesis cases in advance by setting the MGD to the breast phantom equal to $2.5 \mathrm{mGy}$ and exploring the relationships between the MGD and K: 


$$
M G D=K \cdot g \cdot C \cdot S
$$

In this expression, $g, c$ and $s$ are conversion parameters depending on factors such as the breast thickness, glandularity and incident spectra. Their values were taken from Dance et al.(Dance et al 2000a, Dance et al 2009, Dance et al 2011). For instance, in the case of 17 $\mathrm{keV}$, it was calculated that a $4 \mathrm{~cm}$ thick breast-like phantom composed of PMMA corresponds to a breast of $4.34 \mathrm{~cm}$ thickness and a breast composition of 50\% adipose and 50\% glandular tissue. For this specific case, $\mathrm{K}$ was calculated to be $8.7 \mathrm{mGy}$. In the case of tomosynthesis, the total air kerma was again $8.7 \mathrm{mGy}$ and was equally divided between the projections in each acquisition set (Dance et al 2011).

\section{Reconstruction methods}

In this study, we reconstructed the volumes using simple and filtered MPA. Filtering prior to the reconstruction is needed in order to compensate for the oversampling that occurs at lower radial frequencies in the Fourier domain and to reduce artifacts and noise that are present in experimental images. A median filter, a sinc filter and a combination of those two filters were used prior to MPA reconstruction. These algorithms are denoted with FMPA-M, FMPASINC and FMPA-MSINC, respectively. The sinc filter has a varying response depending on the value of parameter $a$ (Eq. 4.1). As mentioned in chapter 4, for a value of $a$ close to zero, the sinc filter approaches the standard ramp filter and acts as a simple high-pass filter that is widely used prior to backprojection reconstruction. As $a$ increases, a smoother high-frequency roll-off is achieved. We experimented with different values of $a$, for both the $\mu$ Cs and the low-contrast mass, in order to find the optimal value that should be used in each case.

\section{E. Evaluation}

The variation of several image characteristics, such as CNR, C, and ASF, are investigated for the five angular range scenarios and the four reconstruction algorithms.

For the investigated mass, the average value $\bar{\mu}_{f}$ was calculated over a circular ROI inside the feature with a radius of 45 pixels ( $2.5 \mathrm{~mm})$. The properties of the background ( $\bar{\mu}_{b k}$ and $\sigma_{b k}$ ) were evaluated for an area twice the area selected for the mass. The background ROI was defined by the upper boundary of the image and an inner and outer radius of 55 and 85 pixels respectively, measured from the center of the mass.

For the $\mu \mathrm{Cs}$, three background regions of size $100 \times 100$ were defined, close to the two groups $\mathrm{A}$ and $\mathrm{B}$ for the calculation of the average value of the background $\left(\bar{\mu}_{b k}\right)$. The average feature value $\bar{\mu}_{f}$, for the $\mu \mathrm{Cs}$ with size ranging between $250-500 \mu \mathrm{m}$ was calculated 
as the mean value of the pixels in a selected square area inside each feature. In the case of $\mu \mathrm{Cs}$ with size ranging between 170-220 $\mu \mathrm{m}$, an area that contained each feature was defined and the maximum value was extracted. The mean value was then calculated from these values and used as $\bar{\mu}_{f}$.

\subsubsection{Results}

For each arc, four volumes were reconstructed using MPA, FMPA-M, FMPA-SINC and FMPA-MSINC. Tomosynthesis planes were reconstructed with thickness of $1 \mathrm{~mm}$. Each volume was examined individually to determine the planes with reconstructed features and then the performance of each algorithm was evaluated for low and high-contrast details.

\section{A. Reconstruction of low-contrast features}

\section{i. Filter Optimization}

Initially, an optimization study was carried out aiming to find the optimal value for the parameter $a$ of the sinc filter [see Eq. 4.1] used in the reconstruction of the $6 \mathrm{~mm}$ low-contrast features. Tomosynthesis slices containing these features, reconstructed with FMPA-MSINC for the five acquisition arcs and different values of $a$, are shown in Fig. 6.7. 


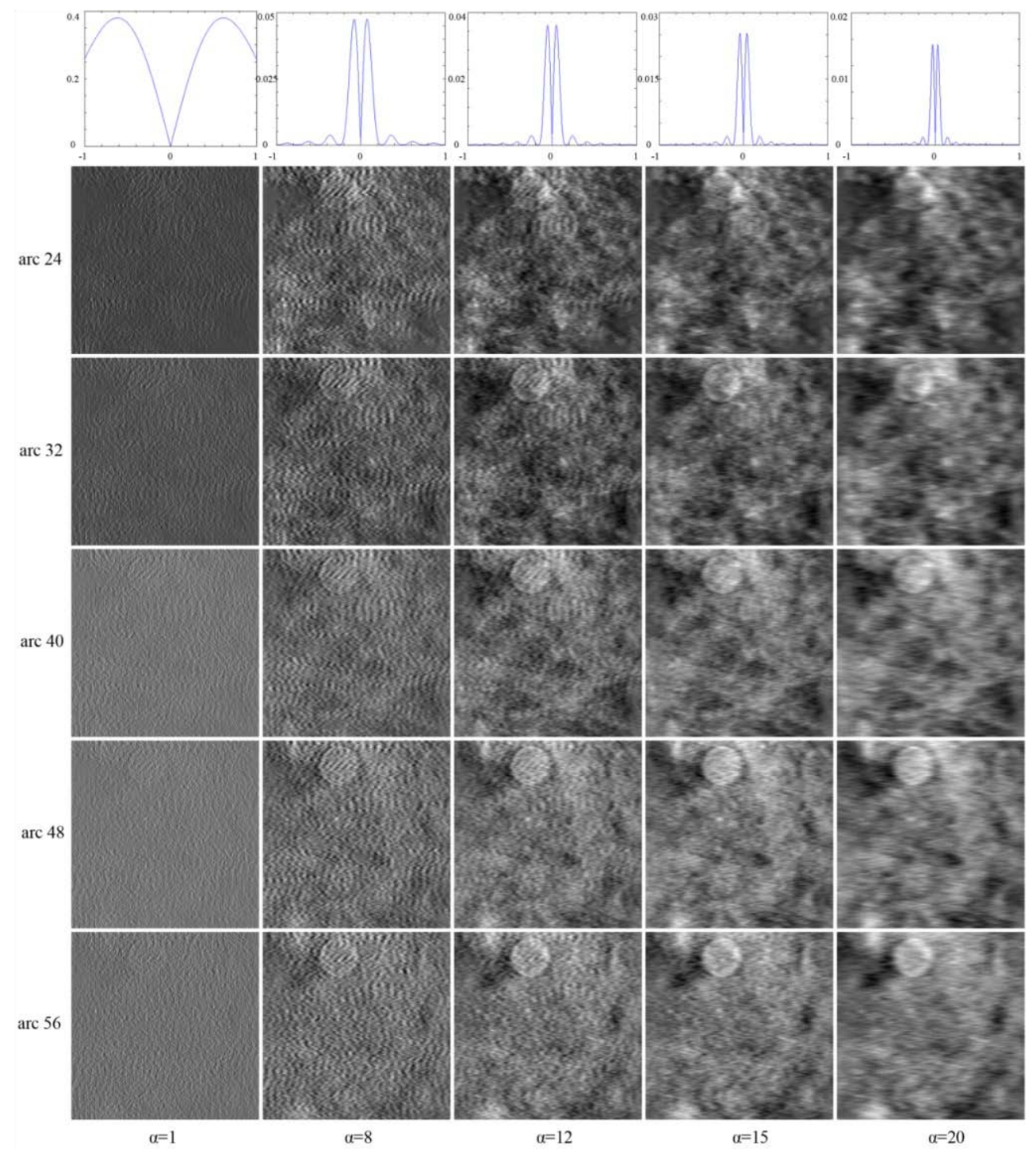

Fig. 6.7. Tomograms that contain low contrast features reconstructed with FMPA-MSINC for five acquisition $\operatorname{arcs}\left(24^{\circ}, 32^{\circ}, 40^{\circ}, 48^{\circ}\right.$ and $\left.56^{\circ}\right)$ and five values of $\alpha(1,8,12,15$ and 20$)$. The first row shows the response of the corresponding sinc filter.

The first row in Fig. 6.7 presents the corresponding sinc filter response for five values of $a$ (1, 8, 1215 and 20) that was used prior to the reconstruction with MPA in order to filter the initial projection images. Reconstructed tomograms with each of the sinc filters used in FMPA-MSINC are shown in the column under the corresponding filter response for each acquisition arc. It is observed that the low-contrast feature detectability improves, i.e. the edges of this object are enhanced, as the value of $a$ is raised (e.g. for $a=12$ ). Further increase of $a$ does not result in additional visual improvement of the low-contrast feature. The presence of excessive blurring as observed in the images from the last column $(a=20)$ in Fig. 6.7 leads to poor image quality of the reconstructed object. Finally, for all $a$ values, the visibility of the low-contrast details is enhanced with the increase of the arc length. 
In addition to the visual assessment of reconstructed low-contrast features, the CNR values were calculated and used to find the optimal $a$ parameter. CNRs of low-contrast features were calculated for 21 filter configurations for the two algorithms that exploit the sinc filter. The first $a$ value used was 0.1, resulting in a typical ramp filter response, and then $a$ varied from 1 to 20, changing the filter's response as shown in the first row of Fig. 6.7. Figure 6.8 shows the CNR results of tomograms containing the $6 \mathrm{~mm}$ low-contrast feature under evaluation [see Fig. 6.6(d)], for projection images acquired within an acquisition arc of $40^{\circ}$. CNR increases substantially with $a$ value up to $a=12$. Beyond this value, the improvement rate decreases and for $a$ higher than 15, CNR remains almost constant. Similar trends in the CNR curves with $a$ were observed for low-contrast features on tomograms obtained for the other four angular ranges.

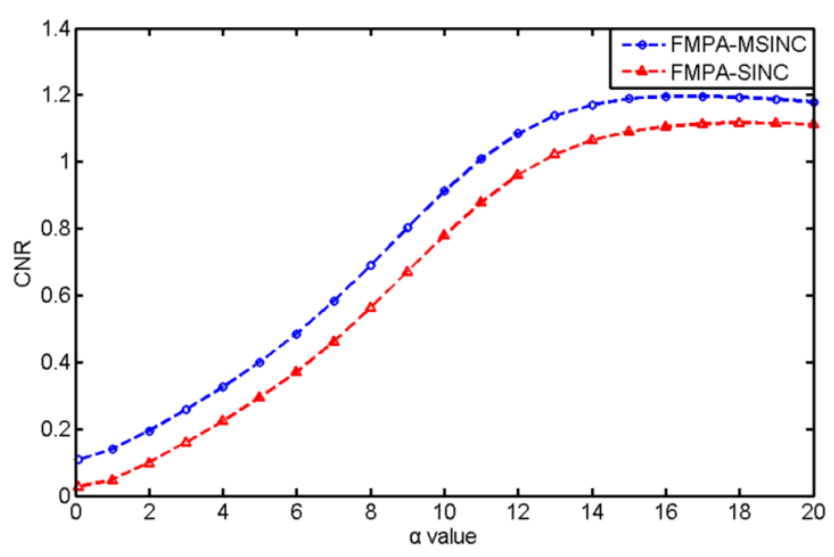

Fig. 6.8. Filter optimization for low contrast feature detection. CNR as a function of $\alpha$ sinc filter parameter for the two algorithms FMPA-SINC and FMPA-MSINC. Data for acquisition arc $40^{\circ}$.

The optimal parameter $a$, for $6 \mathrm{~mm}$ low-contrast feature reconstructed with FMPA-SINC and FMPA-MSINC was set to $a=12$ and was defined by combining the results from the quantitative assessment (Fig. 6.8) and the visual appearance of the low-contrast details on tomograms (Fig. 6.7). The results presented in the rest of the current study for the lowcontrast mass are calculated for this value.

\section{ii. Evaluation of image quality as a function of the acquisition arc}

Tomosynthesis slices with low-contrast features, reconstructed using the four versions of MPA for the five acquisition arcs, are shown in Fig. 6.9. Low contrast and poor detectability are observed in the case of simple MPA and FMPA-M, while images reconstructed with FMPA-MSINC and FMPA-SINC demonstrate increased feature visibility. 


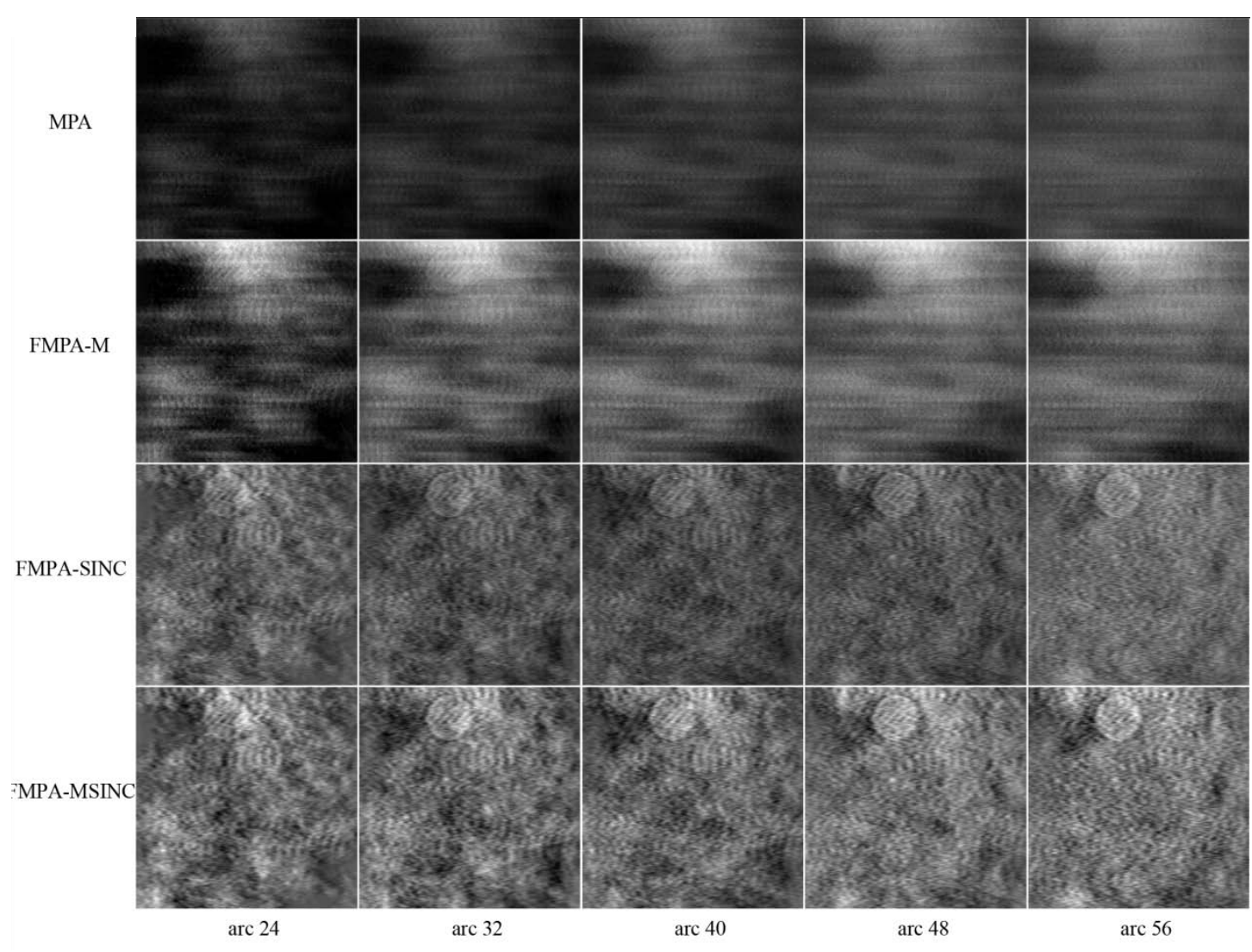

Fig. 6.9. Tomograms that contain low contrast features reconstructed with simple MPA (first row), FMPA-M(second row), FMPA-SINC (third row) and FMPA-MSINC (fourth row) for five acquisition arcs $\left(24^{\circ}, 32^{\circ}, 40^{\circ}, 48^{\circ}\right.$ and $\left.56^{\circ}\right)$.

The image quality of the low-contrast feature, reconstructed with the four algorithms, was further assessed against the angular range. Figures 6.10(a) and 6.10(b) present the CNR and C values as a function of the acquisition arc for this feature reconstructed with MPA, FMPA-M, FMPA-SINC and FMPA-MSINC. Higher CNR and C values were observed for the two filtered MPA algorithms that exploit the sinc filter. Moreover, CNR and C increase with the arc length for these two reconstruction algorithms. The improvement is also visually noticed in the images, in the two lower rows of Fig. 6.9. On the other hand, the CNR and C values are almost constant for MPA and FMPA-M with the extension of the arc length. 


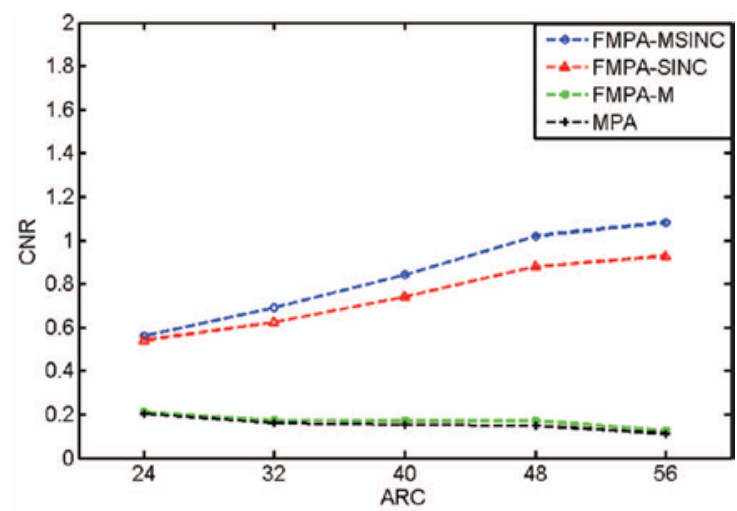

(a)

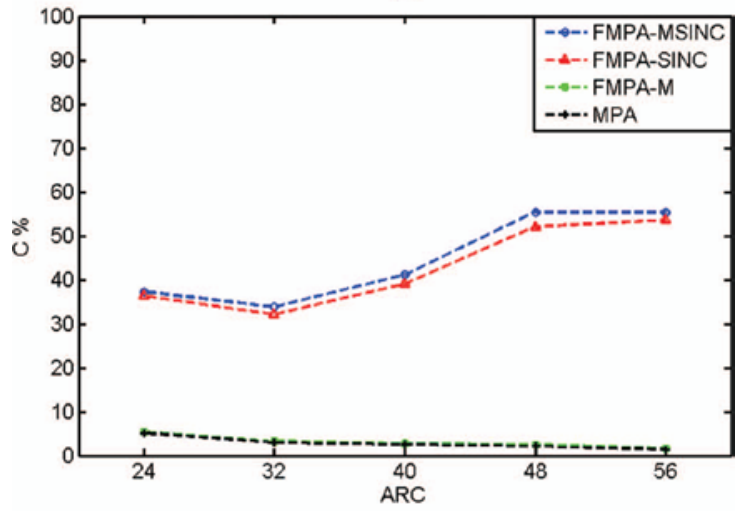

(b)

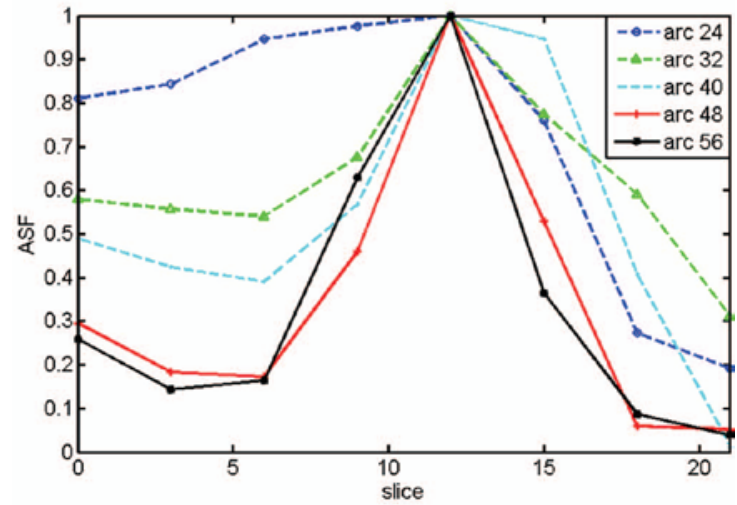

(c)

Fig. 6.10. Quantitative assessment of the $6 \mathrm{~mm}$ low-contrast feature on reconstructed tomograms: (a) CNR and (b) C for all four reconstruction algorithms and (c) ASF for all five acquisition sets reconstructed with FMPA-MSINC.

Finally, the ASF curves for the five acquisition sets using FMPA-MSINC are depicted in Fig. 6.10(c). As expected, increasing the acquisition arc results in improved z-axis resolution and better low-contrast differentiation. 


\section{B. Reconstruction of small high-contrast features}

\section{i. Filter Optimization}

Similarly to the low-contrast feature case, the FMPA-SINC and FMPA-MSINC algorithms were optimized to reconstruct the $\mu \mathrm{Cs}$ with optimal image quality. Tomograms, containing the two groups of $\mu \mathrm{Cs}$ (A and B) reconstructed with FMPA-MSINC for different $a$ values using projection images acquired at $24^{\circ}$, are shown in Fig. 6.11. Similar images were obtained for the rest of the acquisition arcs.
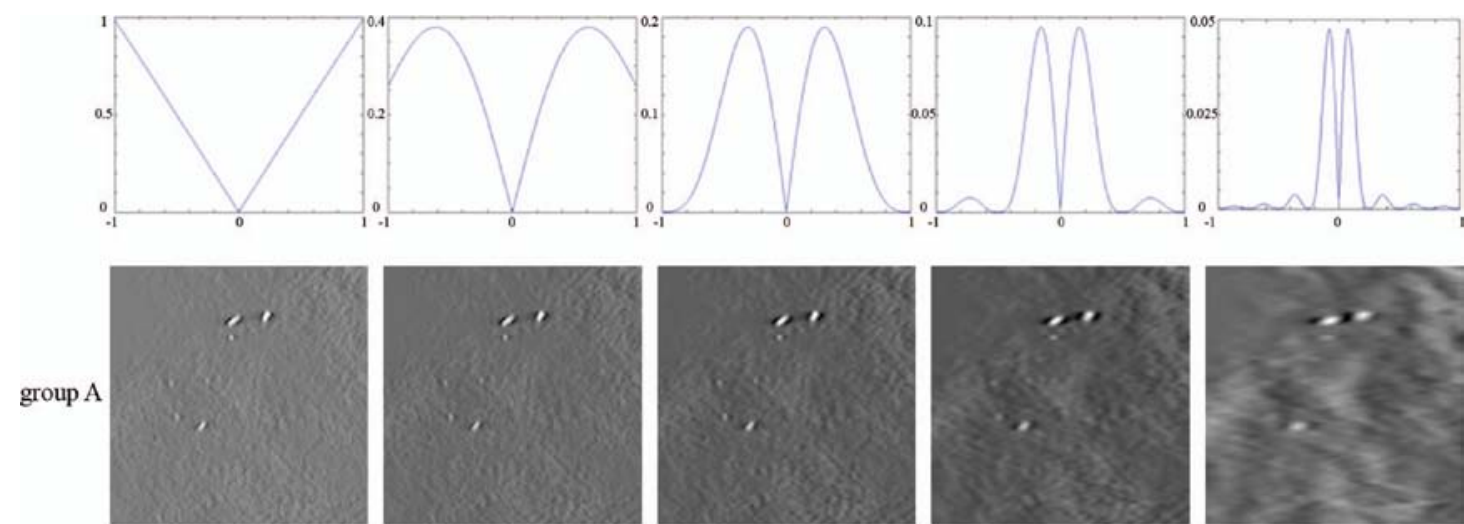

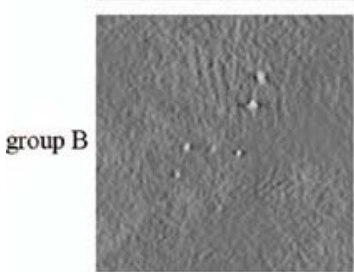

$\alpha=0.1$

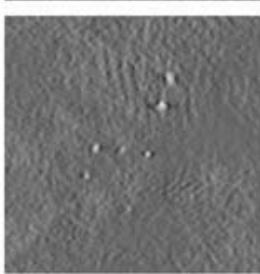

$\alpha=1$

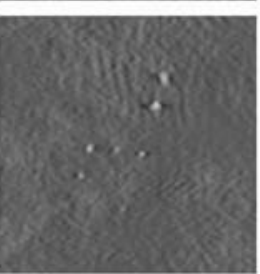

$\alpha=2$

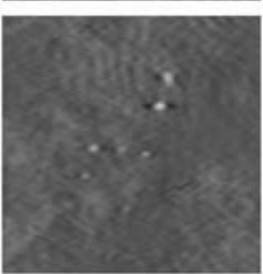

$\alpha=4$

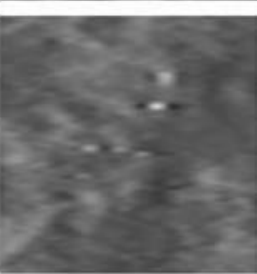

$\alpha=8$

Fig. 6.11. Regions of interest from tomograms that contain two groups (A and B) of $\mu$ Cs reconstructed with FMPA-MSINC for five values of a $(0.1,1,2,4$, and 8$)$ for an arc of $24^{\circ}$. The first row shows the response of the corresponding sinc filter.

For the $\mu \mathrm{Cs}$, low $a$ values (e.g. $a \leq 1$ ) result in images with features having sharper edges due to the suppressed low frequency background. As $a$ increases, shadow artifacts around the $\mu \mathrm{Cs}$ become more visible, while for bigger values (e.g. $a>4$ ) the features get blurry.

Quantitatively, the effect of $a$ parameter on CNR for $\mu$ Cs with sizes ranging between 0.170$0.220 \mathrm{~mm}$ and $0.250-0.500 \mathrm{~mm}$ is presented in Figs. 6.12(a) and 6.12(b) respectively. These curves show optimal CNR values for $a$ in the range between 1 and 3 . In this region, the filter has poor low frequency response and reduces effectively the presence of anatomical background that contains mainly low frequencies. For higher $a$, the CNR values progressively decrease indicating deterioration of image quality. 
The optimal value of $a$ for the FMPA-SINC and FMPA-MSINC algorithms used in reconstruction of $\mu \mathrm{Cs}$ was set to $a=1$. This value was selected by combining the results from the visual appearance of these objects on tomograms (Fig. 6.11) and the quantitative assessment, with more emphasis being given to the results for the $\mu$ Cs with size $0.170-0.220$ $\mathrm{mm}$ [see Fig. 6.12(a)], as they are more difficult to identify and visualize. The results presented in the rest of this study concerning all $\mu \mathrm{Cs}$ are calculated for this $a$ value.

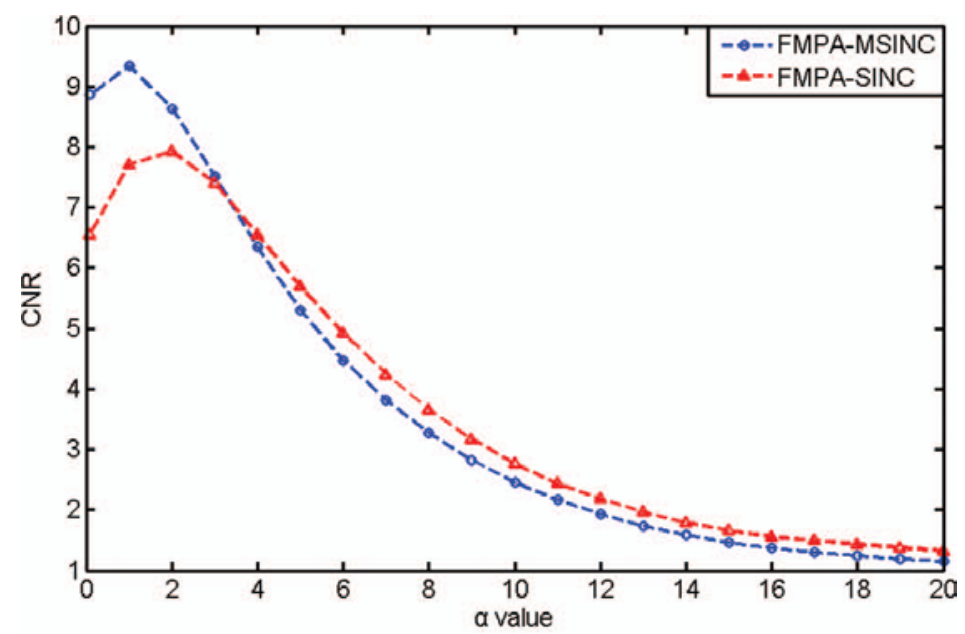

(a)

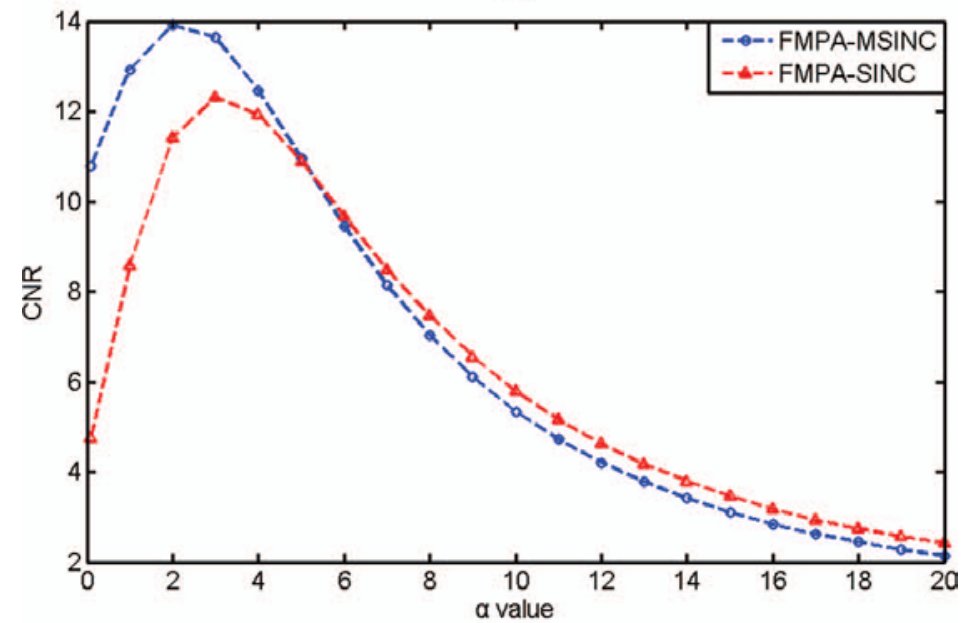

(b)

Fig. 6.12. Filter optimization for detection of $\mu$ Cs. CNR as a function of $\alpha$ sinc filter parameter for (a) $\mu \mathrm{Cs}$ with size $170-220 \mu \mathrm{m}$ and (b) $\mu \mathrm{Cs}$ with size $250-500 \mu \mathrm{m}$. Data for acquisition arc $40^{\circ}$.

\section{ii. Evaluation of image quality as a function of the acquisition arc}

Regions of interest from tomographic slices that contain the two groups of $\mu$ Cs (A and $B$ ), reconstructed with simple MPA, FMPA-M, FMPA-SINC and FMPA-MSINC are summarized in Fig. 6.13 for all acquisition sets. All algorithms were able to reconstruct all $\mu \mathrm{Cs}$. Similarly to the low-contrast feature case, FMPA-MSINC demonstrates remarkably 
better feature appearance due to the good anatomical background suppression. In the case of FMPA-SINC, high-frequency artifacts from other planes result in low contrast appearance of the corresponding images. A median filter applied in advance on projection images eliminates these high frequency elements and at the same time decreases the noise in the initial projections.
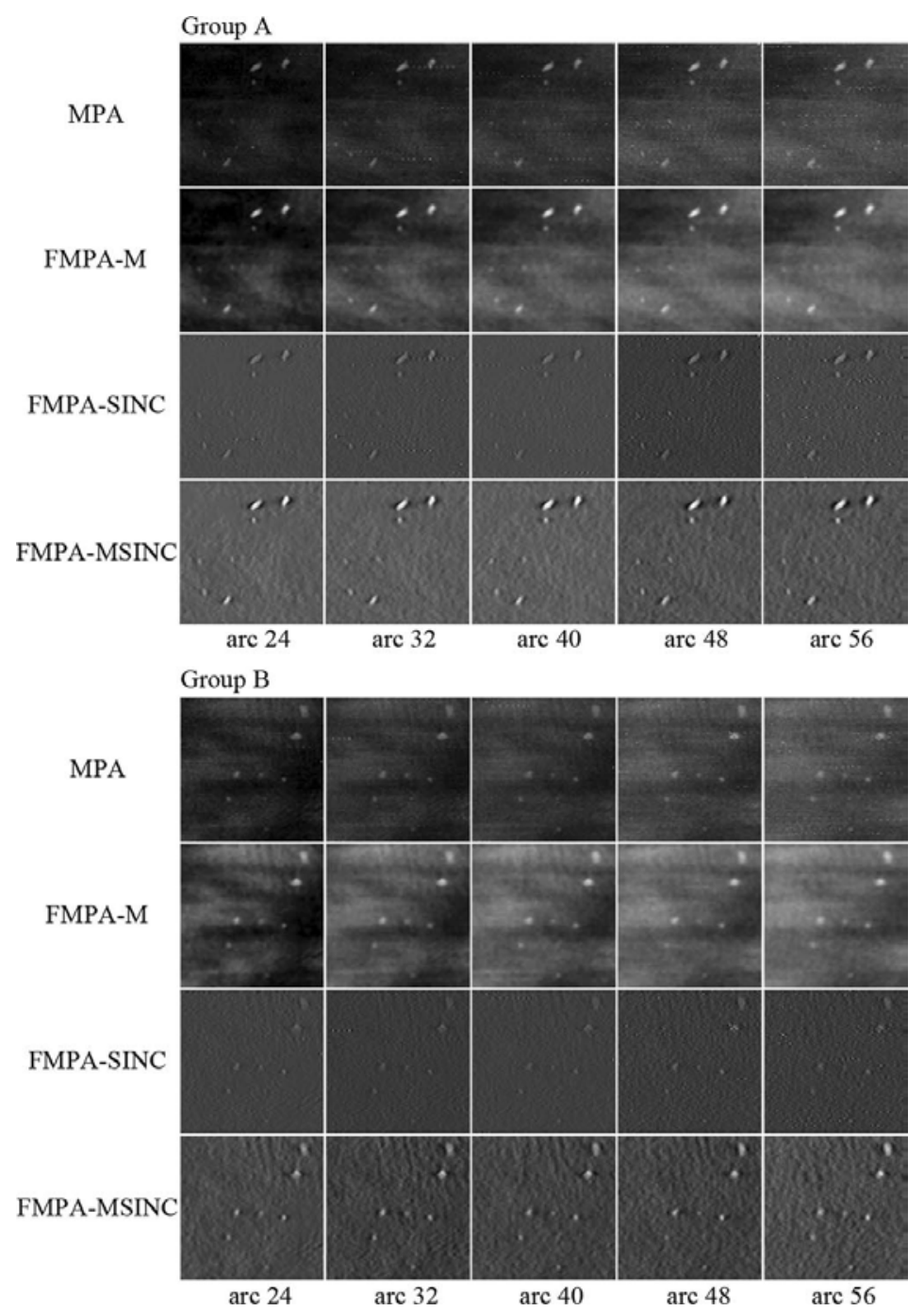

Fig. 6.13. Regions of interest from tomograms that contain two groups (A and B) of $\mu$ Cs reconstructed with simple MPA (first row), FMPAM (second row), FMPA-SINC (third row), and FMPA-MSINC (fourth row) for five acquisition $\operatorname{arcs}\left(24^{\circ}, 32^{\circ}, 40^{\circ}, 48^{\circ}\right.$, and $\left.56^{\circ}\right)$.

The CNR, C and ASF calculated for $\mu$ Cs with sizes ranging between $0.170-0.220 \mathrm{~mm}$ and 0.250-0.500 mm are depicted in Figs. 6.14(a) and 6.14(b), respectively. 

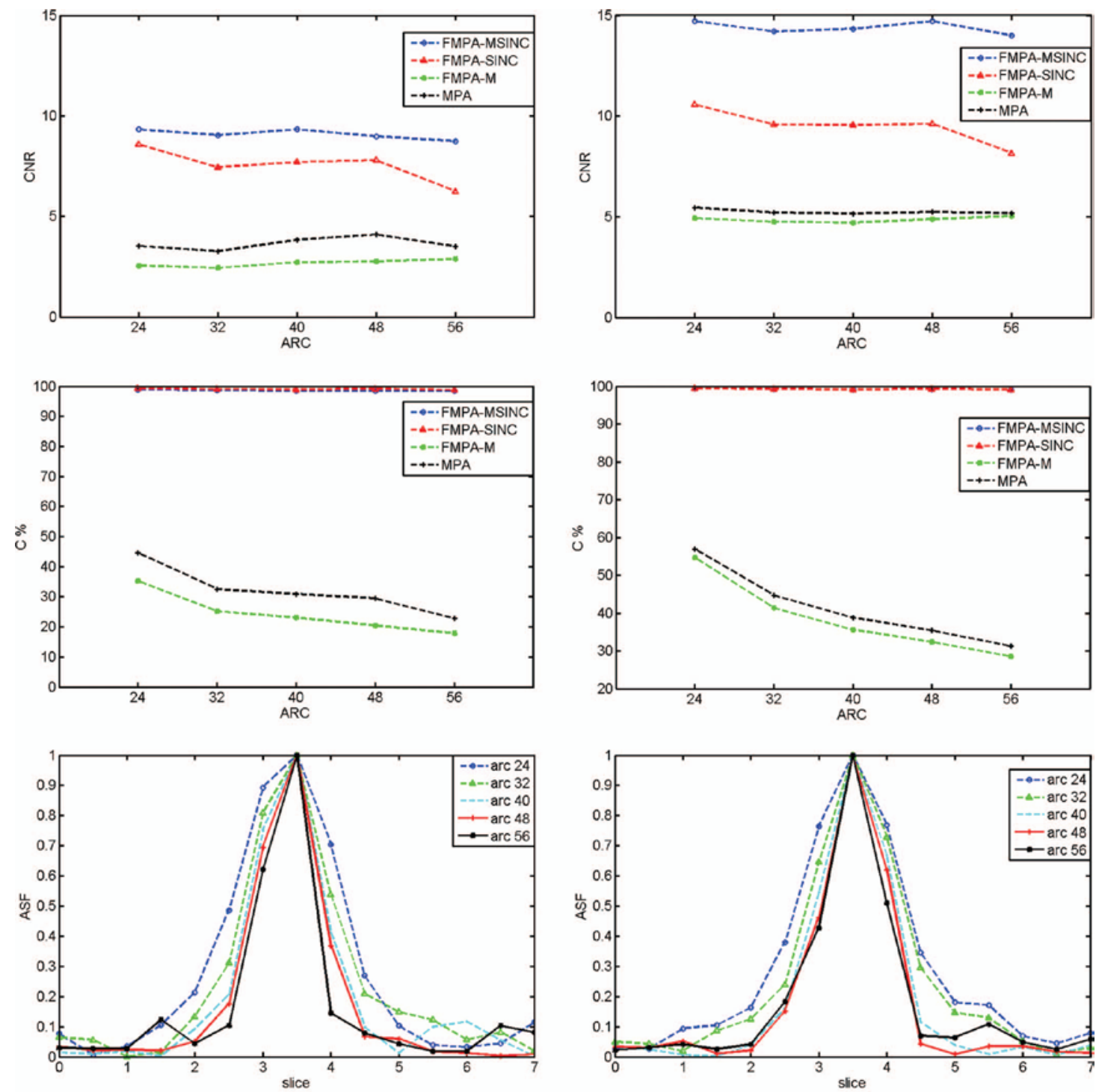

(a)

(b)

Fig. 6.14. Quantitative assessment of $\mu \mathrm{Cs}$ with size (a) $170-220 \mu \mathrm{m}$ and (b) $250-500 \mu \mathrm{m}$ on tomograms: CNR (first row) and C (second row) for all four reconstruction algorithms and ASF (third row) for all five acquisition sets reconstructed with FMPA-MSINC.

The CNR for all high-contrast features remains almost unchanged with extension of the arc from $24^{\circ}$ to $56^{\circ}$, whereas the $\mathrm{C}$ slightly diminishes in the case of simple MPA and FMPA-M. Quantitative evaluation confirms the visual observation concerning the improved performance of FMPA-MSINC in comparison to the other algorithms. For all arcs, it is observed that the CNR values obtained with FMPA-MSINC compared to the corresponding values acquired with simple MPA are about two times higher for the $\mu \mathrm{Cs}$ with sizes between 0.170 and 0.220 $\mu \mathrm{m}$ and about three times higher in the case of $\mu \mathrm{Cs}$ with sizes between 0.250 and $0.500 \mu \mathrm{m}$. Moreover, the $\mathrm{C}$ values are much higher for the two algorithms using the sinc filter compared to the other two. This difference in $\mathrm{C}$ is also noticeable in the images in Fig. 6.13. 
The ASF graphs improve with the extension of the acquisition arc. Similarly to the case of low-contrast features (Sec. 6.2.3.A.ii), less artifacts and better vertical resolution are obtained with larger acquisition arcs.

\subsubsection{Discussion}

The optimization part of the study that aimed to determine the sinc filter's parameter $a$, indicated that low-contrast features are well visualized for $a=12$, while for $\mu$ Cs the optimal value of $a$ was 1 .

For low $a$ values, ranging between 0.1 and 1, the filter's response approaches the typical ramp high-pass filter. In this case, the low frequency background is minimized, while the high frequency content is amplified due to the nature of this filter. For small high-contrast features this filter is a good choice as shown in the first column of Fig. 6.11, since the high frequencies are amplified and at the same time the heterogeneous background is completely suppressed. The features reconstructed with such type of filters are therefore characterized with very high contrast, sharper edges and higher CNR (Fig. 6.12). For low-contrast masses, such as the one evaluated in the current study, the filter has a weaker response in this region ( $a$ between 0.1 and 1). The CNR values are very low due to low frequency suppression and presence of high frequency noise in the reconstructed images (Fig. 6.8). This is also visually observed in the first column of Fig. 6.7, as the low-contrast features are weakly detected for these values of $\alpha$. Similar results were reported in our previous study, in which we used 21 projection images acquired in an acquisition arc of $40^{\circ}$. The filter was not optimized to detect low-contrast structures and actually behaved as a typical ramp filter.(Bliznakova et al 2010a) However, increasing the value of $a$ changes the sinc filter's response minimizing the noise and allowing low-frequency amplification. This behavior results in increased low-contrast feature visibility and improved CNR values optimizing the filter for the reconstruction of these low-contrast objects as well. Excessive increase in $a$ value (e.g. $a>12$ ) though, should be avoided as it results in deterioration of the feature visibility due to blurring.

Therefore, filter optimization prior to the reconstruction is important and shows that the optimal $a$ parameter depends on the feature to be detected, i.e. on its attenuation characteristics and dimensions. The optimal $a$ value should be defined as a trade-off between the feature edge enhancement and the level of presence of the background. This is also illustrated with the detection of the other two low-contrast features on the upper and lower right part of the reconstructed tomograms (Fig. 6.7). These features have very low x-ray contrast and are poorly visualized, even on the single projection image of the $1 \mathrm{~cm}$ TORMAX 
phantom alone [Fig. 6.6(a)]. For low a values, they were suppressed along with the anatomical background and therefore not detected. After filter optimization in combination with extended acquisition arc, they become slightly detectable in the MAMMAX tomograms.

The extension of the acquisition arc results in improved visibility of low-contrast features that are hidden in heterogeneous background, due to the increased resolution in vertical directions. This is well confirmed by the quantitative evaluation. Optimal CNR and C values as well as visualization are obtained for an acquisition arc of $56^{\circ}$ and 15 projection images (Figs. 6.9 and 6.10). The lower CNR and C for FMPA-M and simple MPA can be explained by the blurring that exists in reconstructed images. For the chosen arc and number of projection images combinations, the calculated CNR values for a low-contrast feature reconstructed with algorithms using the sinc filter are in agreement with our simulation study (Malliori et al 2013) and with the results from the simulation study of Van de Sompel (Van De Sompel et al 2011). Similar trends in CNR with the arc were reported in the simulation studies of Sechopoulos and Ghetti using iterative algorithm (Sechopoulos and Ghetti 2009) and other simulation studies with a homogeneous background and filtered backprojection algorithms (Zhou et al 2007, Zhao et al 2009). For small high-contrast features, the arc length is not a critical factor for their visualization, as seen from the inspection of the two groups of $\mu \mathrm{Cs}$ on reconstructed tomograms (Fig. 6.13). Their CNR values (Fig. 6.14) are less sensitive to the length of the acquisition arc, compared to the case of the low-contrast feature. This observation is in agreement with the results from the simulation studies performed by Sechopoulos and Ghetti (Sechopoulos and Ghetti 2009). Although the noise in projection images increases with the arc, due to the increased tissue thickness in the projection, this effect is small compared to the high inherent contrast of the features. However, this effect becomes more critical, in the case of simple MPA and FMPA-M, and results in a decrease in the $C$ values of $\mu \mathrm{Cs}$ with the extension of the acquisition arc. Filtered methods improve the visual appearance of high-contrast structures as pointed out also from a few experimental and simulation studies (Malliori et al 2010, Zhou et al 2007, Zhao et al 2009, Malliori et al 2013).

The ASF analysis for the studied $\mu$ Cs and especially for the low-contrast feature showed, as expected, that the artifacts in adjacent planes decrease with the increase of the arc length. Specifically, for arcs of $48^{\circ}$ (13 projection images) and $56^{\circ}$ (15 projection images), the ASFs for low and high-contrast features improve compared to the ASFs of other arcs, resulting in higher vertical resolution and therefore in better image quality.

Undoubtedly, the greatest advantage of the tomosynthesis technique lies in its potential to reconstruct low-contrast features that are not distinguished in single projection images. Tomosynthesis images are characterized by higher contrast compared to projection images. 
This is due to the ability of tomosynthesis to reconstruct the details in the different planes by reducing the tissue overlap present in the standard planar images. In both simple and filtered MPA, the features of interest are visible. In the case of simple MPA, the reconstruction suffers from severe blurring artifacts that are along the motion direction of the x-ray source. The use of appropriate filtering methods eliminates these artifact effects and suppresses the anatomical background, remarkably improving the feature visualisation. This is valid for both types of low and high-contrast features with the latter having a size ranging between $170 \mu \mathrm{m}$ and $500 \mu \mathrm{m}$.

\subsubsection{Conclusions}

This study evaluates the image quality of breast-like lesions such as masses and $\mu \mathrm{Cs}$ in BT tomograms reconstructed with simple and filtered MPA applied on projection images acquired with monochromatic beams at synchrotron facilities. Tomograms with these features, hidden in the highly heterogeneous breast background, were reconstructed for five acquisition arcs and limited number of projections that varied between 7 and 15 . The $6 \mathrm{~mm}$ low-contrast features are better visualized at extended arc length. The acquisition arc of $56^{\circ}$ with 15 projection images demonstrated the highest image reconstruction quality for the lowcontrast mass based on the results from all figures of merit. The image quality of $\mu \mathrm{Cs}$ is less sensitive to the acquisition arc. Calcifications with size ranging between $170 \mu \mathrm{m}-500 \mu \mathrm{m}$, such as the presently studied high-contrast features, are well identified and visualized in all arcs.

Among the four reconstruction algorithms that were used, FMPA-MSINC has much better performance in all cases. However, filter optimization in respect to the type of feature characteristics is important before the reconstruction. Finally, this study indicated that the application of BT is essential in bringing into focus and localizing low-contrast features. 


\subsection{DUAL ENERGY APPLICATION}

\subsubsection{Introduction}

The application of dual-energy (DE) subtraction imaging techniques in mammography has been investigated in many theoretical and experimental studies (Johns et al 1985, Johns and Yaffe 1985, Chakraborty and Barnes 1989, Boone 1991, Brettle and Cowen 1994, Lemacks et al 2002, Bliznakova et al 2006, Kamarianakis et al 2009, Carton et al 2010) and a number of clinical cases in which DE has demonstrated enhanced visualization compared to conventional mammography (Asaga et al 1987, Asaga et al 1995, Lewin et al 2003). The aim of the current study is to evaluate dual-energy in breast tomosynthesis (DE-BT) using synchrotron radiation in terms of its potential to detect and better characterize low contrast features simulating breast lesions. For this purpose a direct comparison between BT, DE-BT and single acquired projection at the energy of BT application has been performed.

\subsubsection{Materials and Methods}

The experimental study was performed using the MAMMAX complex breast phantom with a thickness that corresponds to $4.3 \mathrm{~cm}$ compressed breast with an average composition of $50 \%$ adipose and 50\% glandular tissue. For DE-BT, a set of 9 projection images, taken at $17 \mathrm{keV}$ and $27 \mathrm{keV}$ for an acquisition arc $32^{\circ}$ was acquired at the SYRMEP beamline at ELETTRA Synchrotron Light Laboratory, Trieste, Italy. For the application of BT, 9 projections images within a $32^{\circ}$ arc were acquired at $17 \mathrm{keV}$. Total MGD was set to $4.3 \mathrm{mGy}$ and $2.5 \mathrm{mGy}$ respectively. For comparison purposes, a planar image taken at $17 \mathrm{keV}$ with an MGD of 2.5mGy was also used. The reconstruction algorithm was the MPA.

Dual-energy projections were produced and the weighting factor $\mathrm{R}$, was calculated theoretically as described analytically in (Bliznakova et al 2006) by the equations:

$$
D E=\ln N_{H}-R \cdot \ln N_{L} \quad \text { and } \quad R=\frac{\mu_{H}}{\mu_{L}}
$$

where $\mathrm{N}_{\mathrm{H}}, \mathrm{N}_{\mathrm{L}}$ and $\mu_{\mathrm{H}}, \mu_{\mathrm{L}}$ are the photons absorbed in the detector and the effective linear attenuation coefficients for the breast tissue, respectively at high and low energy.

The theoretically calculated value for R, was also experimentally verified. For this purpose, different values in the range of $\mathrm{R}=0.2: 2$ using a step of 0.1 were used and evaluated. 
The quality of the images was assessed for a $6 \mathrm{~mm}$ low contrast mass visually and quantitatively in terms of CNR and C. Additionally two more figures of merit were used exploring CNR-to-dose and Contrast-to-dose relationships.

\subsubsection{Results and conclusions}

Results from this study indicated superior performance for the two BT tomographic techniques compared to single projection images for the $6 \mathrm{~mm}$ mass. A 2D planar image of the phantom is depicted in Fig. 6.15(a), while the tomograms of the same phantom reconstructed with BT and DE_BT are shown in Figs. 6.15(b) and 6.15(c), respectively.

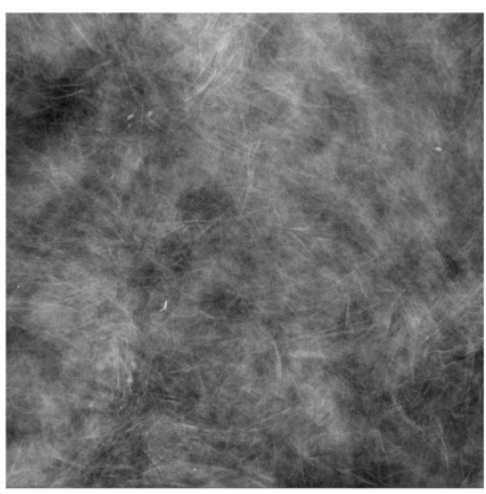

(a)

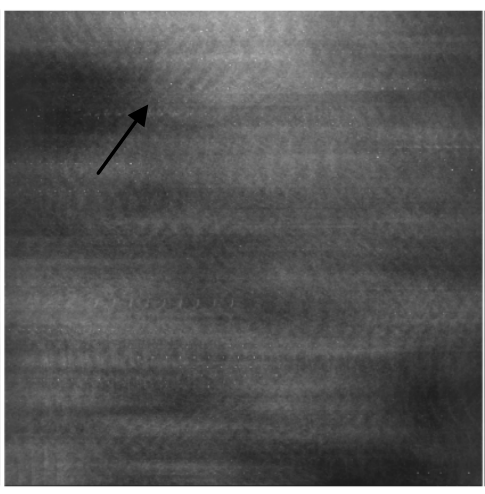

(b)

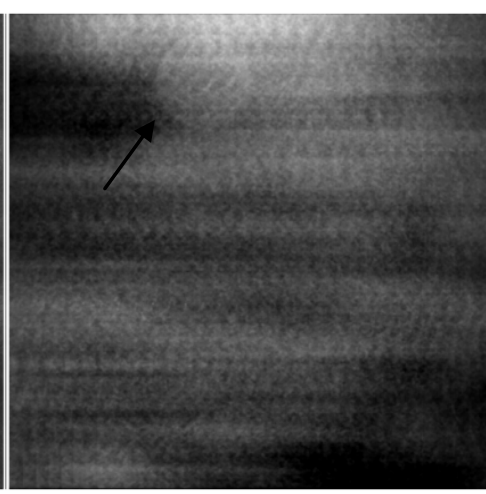

(c)

Fig. 6.15. MAMMAX phantom: (a) 2D planar image (b) BT and (c) DE_BT tomograms of the slice with the low-contrast feature in-focus.

In the planar image the low-contrast mass was not identified at all due to the superimposition of tissue, while it could be well detected in the tomograms, especially in the image obtained with application of dual energy subtraction technique on the projections along with tomosynthesis (DE_BT). The latter, with an appropriately optimized weighting factor R, resulted in better image quality and evaluation metrics compared to simple BT. The value of the weighting factor $\mathrm{R}$ that was theoretically calculated (Eq. 6.2) was found equal to 0.7 and was also experimentally verified.

The graph in Fig. 6.16 represents the Contrast-to-dose results for both methods. For $\mathrm{R}=0.7$, all figures of merit reached their highest value, indicating better image quality for the dual energy projections. The values of all figures of merit for the two methods are summarized in Table 6.4. 


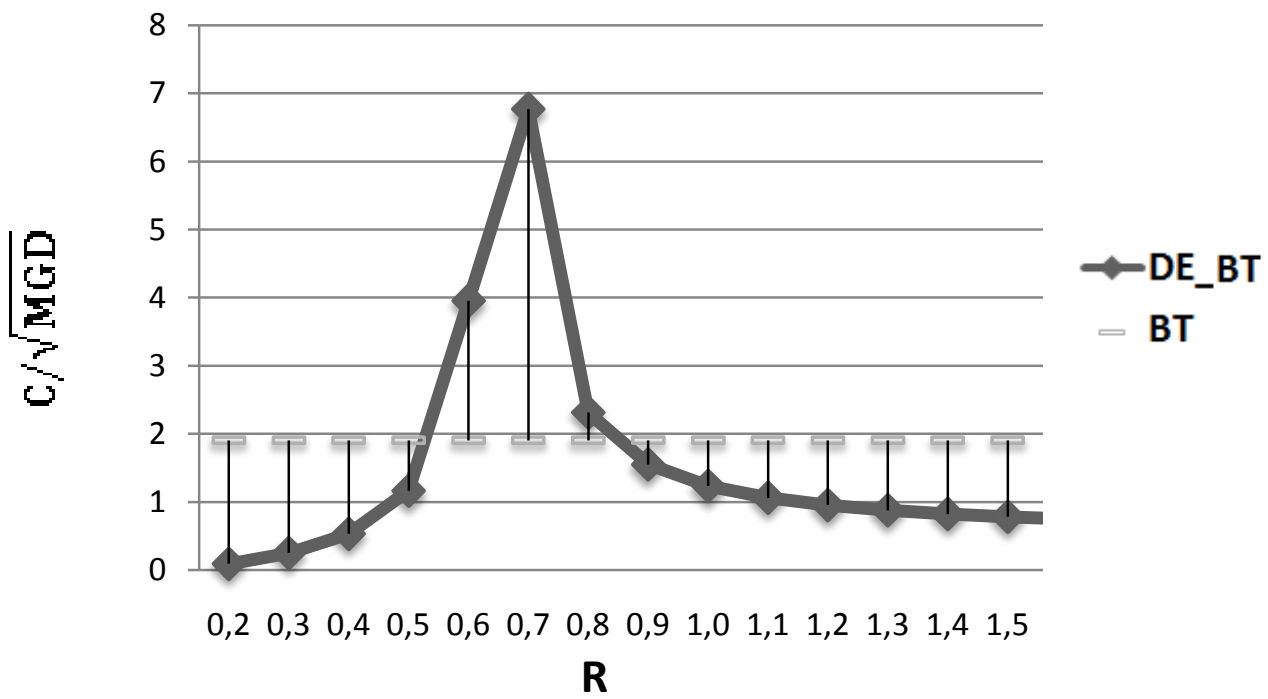

Fig. 6.16. Contrast-to-dose values for different weighting factors R in case of BT and DE_BT.

Table 6.4. CNR, C, CNR-to-dose and Contrast-to-dose values for DE_BT and BT

\begin{tabular}{ccccc}
\hline & CNR & C & CNR $/ \sqrt{\text { MGD }}$ & C $/ \sqrt{\text { MGD }}$ \\
\hline DE_BT & 0.20 & 14.05 & 0.10 & 6.77 \\
\hline BT & 0.16 & 3.02 & 0.10 & 1.91 \\
\hline
\end{tabular}

Quantitative evaluation shows an advantage for tomosynthesis applied in combination with dual-energy verifying the visual observations (Fig. 6.15). In particular, Contrast-to-dose is more than 3 times higher in DE_BT compared to conventional BT (Table 6.4). 


\subsection{MONOENERGETIC vs POLYENERGETIC: EXPERIMENTAL STUDIES}

\subsubsection{Experiment}

In order to evaluate the benefits of using monochromatic beams for imaging the breast, an experimental study was performed that included a comparison of a planar image acquired with monochromatic beam at $17 \mathrm{keV}$ and planar images that correspond to an incident $\mathrm{x}$-ray spectrum with $\mathrm{W}$ target and $\mathrm{Rh}$ filter of $0.05 \mathrm{~mm}$. The simulation approach of the polychromatic spectrum using monochromatic beams was chosen instead of an experiment due to difficulties in reproducing similar experimental conditions using a clinical system. More specifically, planar images at eight incident energies (14, 16, 18, 20, 22, 24, 26 and 27 $\mathrm{keV}$ ) were used to synthesize the polychromatic images. These sets of measurements were acquired at Elettra Synchrotron facilities in addition to the experimental work described in the previous section (Sec. 6.2) using the same complex MAMMAX phantom (Fig. 6.6).

Photon fluence distribution per incident energy for the x-ray spectrum that corresponds to $32 \mathrm{kVp}$ (W/Rh) (Fahrig and Yaffe 1994) is shown in Fig. 6.17(a). Due to limitations of the system at the SYRMEP, images with incident energy less than $14 \mathrm{keV}$ and higher than $27 \mathrm{keV}$ were difficult to obtain. Thus, images over the region $14 \mathrm{keV}$ to $27 \mathrm{keV}$ were acquired with recalculated photon fluence, shown in Fig. 6.17(b). An incident air kerma, K, of 14 mGy was measured at the surface of the complex phantom. Two additional images corresponding to $32 \mathrm{kVp}(\mathrm{W} / \mathrm{Rh})$ and $23 \mathrm{kVp}(\mathrm{W} / \mathrm{Rh}) \mathrm{x}$-ray spectra were synthesized from the planar images acquired at incident energies in the range 14 to $27 \mathrm{kVp}$, with half the initial incident exposure (7mGy)using an algorithm for producing reduced dose images (Bliznakova et al 2010a). Further, to allow the comparison of CNR and C results derived from different measurements, as in the case of polychromatic and synchrotron beams, the FOM (as described in Eq.2.4) was calculated. This allowed studying the CNR-to-dose relationship in the cases of monochromatic and polychromatic beams. For the conversions between K and MGD, Eq.6.1 was used. 


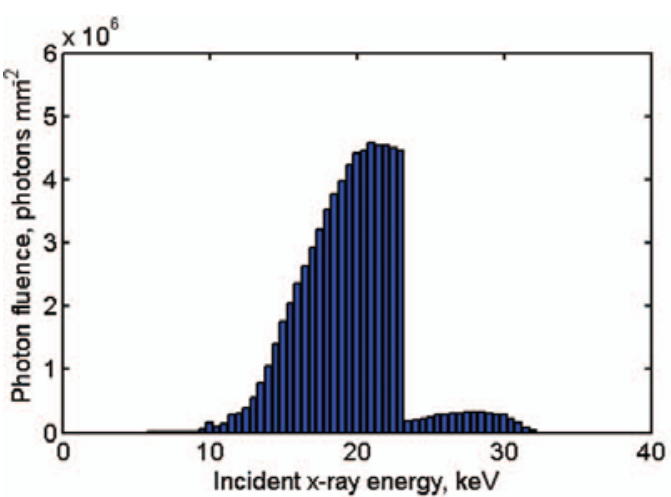

(a)

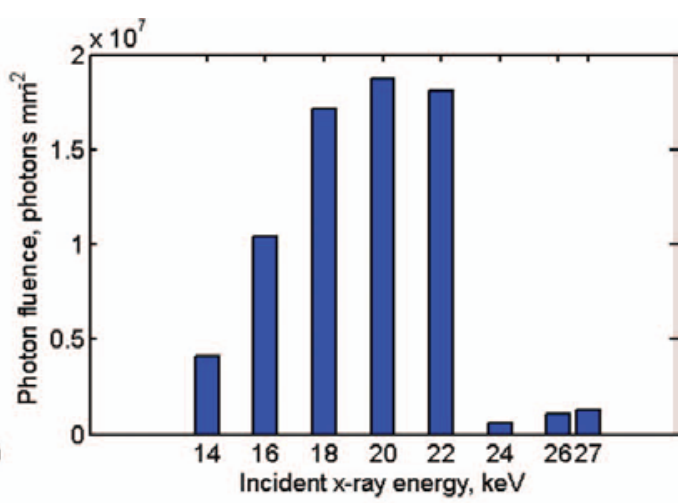

(b)

Fig. 6.17. X-ray spectra used for polychromatic images. (a) Original x-ray spectrum that corresponds to $32 \mathrm{kVp}(\mathrm{W}, 0.050 \mathrm{~mm} \mathrm{Rh}$ ) and (b) spectrum simulated at ELETTRA for the $32 \mathrm{kVp}$ (W, $0.050 \mathrm{~mm}$ $\mathrm{Rh})$ generated with eight energies.

\subsubsection{Results}

Image quality of $\mu \mathrm{Cs}$ in a planar image obtained with a monochromatic beam of $17 \mathrm{keV}$, with an incident exposure of 8.7 mGy resulting in MGD of $2.5 \mathrm{mGy}$, was compared with that of planar images synthesized to correspond to (a) a polychromatic beam of $32 \mathrm{kVp}(\mathrm{W} / \mathrm{Rh}$ ) with incident exposures of 14 and $7 \mathrm{mGy}$ corresponding to MGD of 5 and $2.5 \mathrm{mGy}$, respectively and (b) a polychromatic beam at $23 \mathrm{kVp}$ (W/Rh) with incident exposure corresponding to MGD of 2.5mGy. Images were assessed in terms of CNR, C and FOM and their values for $\mu \mathrm{Cs}$ with size $0.250-0.500 \mathrm{~mm}$ are summarized in Table 6.5. Microcalcifications with smaller size were not quantitatively assessed for the planar images, as most of them could not be easily distinguished from the highly heterogeneous background. For comparison purposes, CNR, C and FOM were also calculated for the image reconstructed with simple (unfiltered) MPA from 7 projections acquired in an arc of $24^{\circ}$. As shown in Table 6.5, the FOM calculated for the single planar image obtained with the monochromatic beam was found to be higher than that of the polychromatic images. Even at double incident exposure the polychromatic image shows comparable CNR value with the monoenergetic one. At the same time, the $\mathrm{C}$ of $\mu \mathrm{Cs}$ was found to be higher compared to that of all the polychromatic images. 
Table 6.5. CNR, C, and FOM evaluation for $\mu \mathrm{Cs}$ in planar images using polychromatic W/Rh (32 kVp, 23kVp) and monochromatic (17 keV) beams. Data evaluated for a tomosynthesis image reconstructed from seven unfiltered projection images acquired for an arc of $24^{\circ}$, are presented in the last row ofthis table.

\begin{tabular}{lcccc}
\hline \multicolumn{1}{c}{ Type } & Energy & CNR & C & FOM \\
\hline PolychromaticMGD=5mGy & 32kVp W/Rh & 2.35 & 8.72 & 1.05 \\
PolychromaticMGD=2.5mGy & $32 \mathrm{kVp} \mathrm{W/Rh}$ & 2.12 & 8.00 & 1.34 \\
PolychromaticMGD=2.5mGy & $23 \mathrm{kVp} \mathrm{W/Rh}$ & 2.33 & 8.34 & 1.47 \\
MonochromaticMGD=2.5mGy & $17 \mathrm{keV}$ & 2.55 & 11.54 & 1.62 \\
TomosynthesisMGD=2.5mGy & $17 \mathrm{keV}$ & 5.46 & 56.83 & 3.95 \\
\hline \hline
\end{tabular}

For equal MGD of 2.5 mGy the comparison between the monochromatic and the polychromatic image indicates again superior image quality in terms of CNR and C for the former. Specifically, the $\mathrm{C}$ of $\mu \mathrm{Cs}$ in the monochromatic image was approximately 1.4 times higher than that of the polychromatic images. Higher CNR and C were found for the monochromatic BT image, reconstructed with simple MPA compared to the planar images.

Figures 6.18(a) and 6.18(b) depict the comparison between the planar image obtained at 17 $\mathrm{keV}$ and the composed image that corresponds to a polychromatic spectrum at $23 \mathrm{kVp}$ (W/Rh), with both images acquired at incident exposure corresponding to MGD of $2.5 \mathrm{mGy}$. Visual comparison of monochromatic and polychromatic images shows contrast enhancement of $\mu \mathrm{Cs}$ in the case of monochromatic beam. In the case however of simple MPA [Fig. 6.18(c)], the visualization of the $\mu \mathrm{Cs}$, especially of those smaller than $220 \mu \mathrm{m}$ is much improved as a result of the reduced variation in the anatomical background around the features. In addition, planar images were unable to visualize or detect the low-contrast mass in the breast phantom. This low-contrast feature is visualized on tomosynthesis images at extended arc (i.e. $>40^{\circ}$ ) even when simple MPA is used (Fig. 6.9, first row) and therefore, uniquely localized in the 3D phantom. 


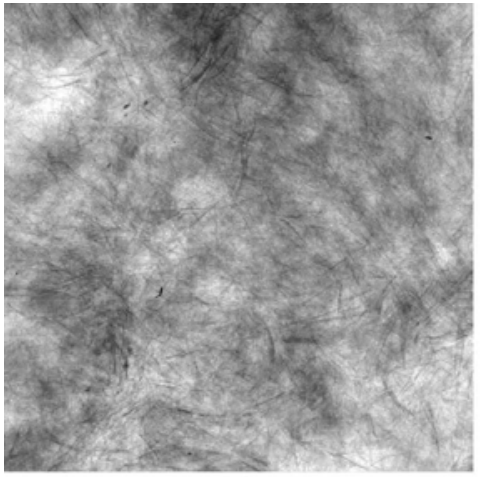

(a)

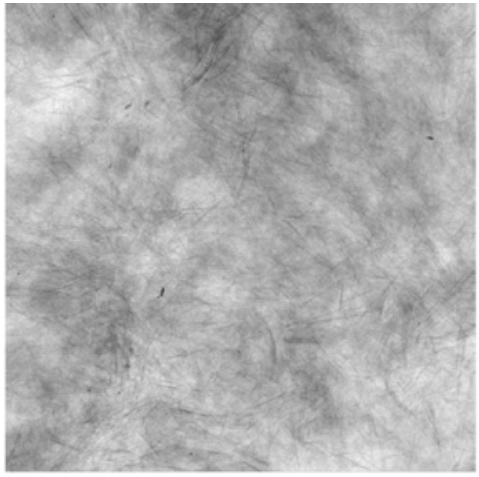

(b)

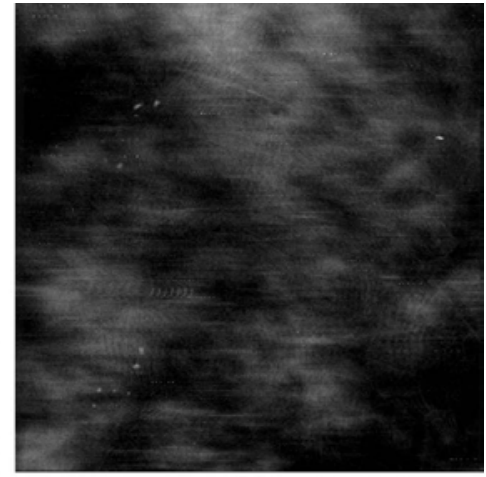

(c)

Fig. 6.18. Planar images acquired at (a) $17 \mathrm{keV}$, (b) $23 \mathrm{kVp}$ (W, $0.050 \mathrm{~mm} \mathrm{Rh}$ ), and (c) tomosynthesis plane that contains $\mu \mathrm{Cs}$ reconstructed with simple MPA. In the last case, the plane is reconstructed from images acquired with an arc of 24. Images correspond to MGD=2.5 mGy.

The results from the comparative study between images obtained from monochromatic and polychromatic beams suggest that the use of monochromatic beams can produce images with higher CNR and C acquired with half incident exposure. This fact could be used in a future tomosynthesis system based on monochromatic beams that may result in tomographic images of higher contrast at lower incident exposures. In addition, monochromatic x-ray beams would potentially allow differentiation of very small attenuation differences in tissue, since better separation between linear attenuation values can be achieved. This is especially valuable in the case of $3 \mathrm{D}$ imaging techniques, as $\mathrm{BT}$, where quantitative information from the voxels of the reconstructed volume may be obtained.

\subsection{MONOENERGETIC vs POLYENERGETIC: SIMULATION STUDIES}

\subsubsection{Introduction}

In general, average glandular doses for a single BT acquisition are reported to vary between 0.6 to 4.0 mGy (Feng and Sechopoulos 2012, Poplack et al 2007, Dobbins 2009). However, the radiation dose depends not only on the exam itself, but also on whether the tomosynthesis exam will be combined with mammography. As a screening technology, reducing the radiation dose required for $\mathrm{BT}$ acquisition with no loss or even improvement of image quality is always desirable. One way to obtain this is through the use of monochromatic beams with BT as pointed out in Chapter 3, section 3.2. Simulation and experimental studies that evaluate directly the feasibility of BT with monochromatic beams have not been reported up to date. In particular, critical in this assessment, is the inclusion of noise from both anatomical and quantum noise sources, as both limit mass conspicuity in clinical images. 
Therefore, the aim of this study is to investigate the impact of using monochromatic beams on image quality for lower dose BT. For this purpose, we performed modeling and simulation of BT and mammography imaging processes and evaluated the results in terms of CNR, C and FOM equal to the CNR normalized by the square root of the MGD (Eq. 2.4). X-ray transport in the breast models was simulated with earlier developed and validated in-house Monte Carlo simulation models. Specifically, the study included simulations with: (a) a mammographic $\mathrm{x}$-ray spectrum $28 \mathrm{kVp}$ W/Rh and monochromatic $\mathrm{x}$ rays with energy of 19 $\mathrm{keV}$ for four incident photon fluence that resulted in entrance surface air kerma (ESAK) in the range 0.16 to $5.5 \mathrm{mGy}$, (b) two software breast models in compressed state with an average thickness of $4 \mathrm{~cm}$ that differ in their complexity and content, and (c) x-ray mammography and BT acquisitions.

\subsubsection{Simulations}

\section{A. Software Phantoms}

\section{i. Simple breast phantom}

A $4 \mathrm{~cm}$ thick software phantom with a homogenous background of $50 \%$ glandular and $50 \%$ adipose tissue has been designed (Fig. 6.19(a)) with the in-house developed software XRayImagingSimulator. Figure 6.19(b) shows an ideal projection image of the square phantom obtained with a $19 \mathrm{keV}$ monochromatic beam, showing the features of interest that are placed in the middle plane of the phantom (at $20 \mathrm{~mm}$ above the phantom base). These features are Cs and breast masses of various sizes and densities. Their characteristics are described in detail in Table 6.6.

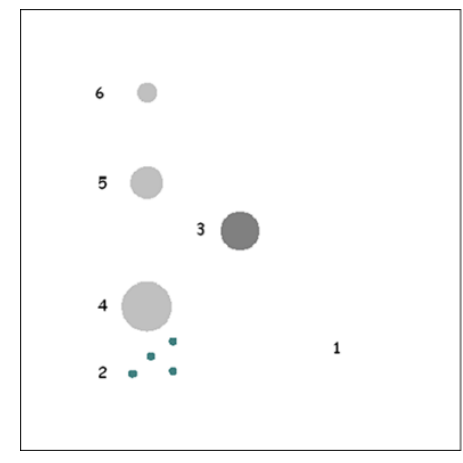

(a)

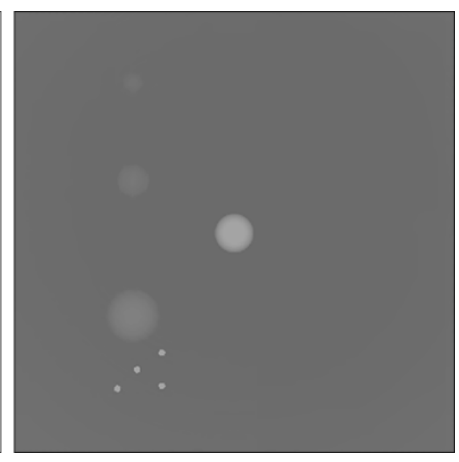

(b)

Fig. 6.19. Simple phantom: (a) Schematic presentation of the arrangement of features in the phantom. Features 3-6 are low-contrast objects that approximate breast masses, 2 is a group of high-contrast objects that represent Cs, 1 is the breast tissue of the homogeneous phantom of $50 \%$ glandularity (dimensions are specified in detail in Table 6.6); (b) noise-free projection image of the phantom. 


\section{ii. CT breast phantom}

A 3D software breast model with realistic breast tissue distribution was created from slices, obtained from a dedicated breast CT scanner at the Department of Radiology and Imaging Sciences at Emory University (Atlanta, Georgia, USA). Initially, the data were de-noised and segmented in order to obtain a compositional breast model composed of skin, glandular, and adipose tissues (Yang et al 2011, Yang et al 2012). The voxel size of the 3D breast model was $0.28 \mathrm{~mm}$ in each direction; the whole breast volume was calculated to be $380 \mathrm{ml}$, placed in a 3D matrix with size of $175 \times 48 \times 134 \mathrm{~mm}^{3}$. The glandular portion occupied 37\% of the breast volume. A compressed version was created from this volume using the compression algorithm (Zyganitidis et al 2007). The compression plates were placed at a position to generate a breast with $4 \mathrm{~cm}$ compressed thickness. For densities $\rho_{\text {gland }}=1.04 \mathrm{~g} / \mathrm{cm}^{3}$ and $\rho_{\text {adipose }}=0.93 \mathrm{~g} / \mathrm{cm}^{3}$, (Hammerstein et al 1979) the mixture by weight of glandular and adipose tissue for this phantom was found to be $40 \%$ and $60 \%$, respectively. Further, four clusters of Cs and a water insert simulating a breast mass with irregular margins were inserted within the 3D computational breast model, all placed in the middle of the phantom (at $20 \mathrm{~mm}$ above the phantom base). Figure 6.20(a) presents schematically the arrangement of the breast abnormalities inserted in the volume, while Fig. 6.20(b) shows a noise-free projection image of the breast phantom obtained with a monochromatic beam of $19 \mathrm{keV}$, using the analytical module of the XRayImagingSimulator (Bliznakova et al 2010b). This projection image depicts the breast tissue, the breast lesion and four groups of Cs with gradually decreasing diameters of 1.3, 1, 0.4, $0.2 \mathrm{~mm}$. The characteristics of the phantom are summarised in Table 6.6.

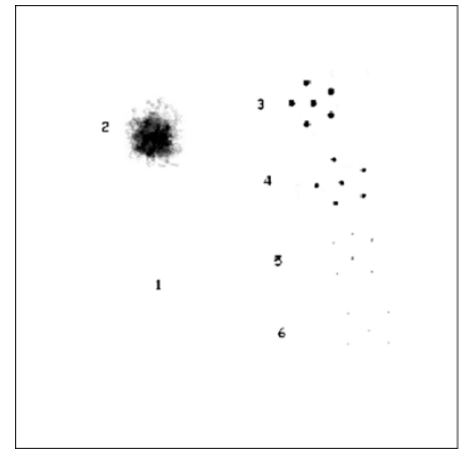

(a)

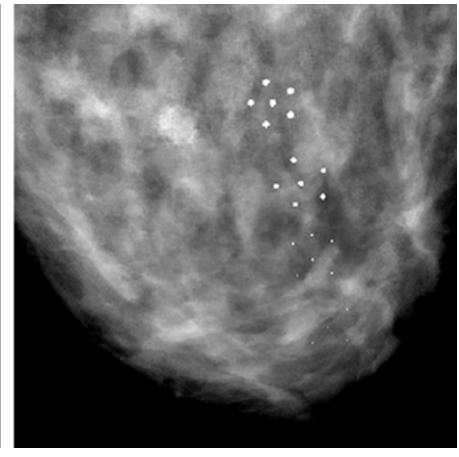

(b)

Fig. 6.20. Software breast model from patient breast CT slices: (a) Schematic representation of abnormalities included in the phantom: 2 is a water insert representing an irregular breast mass, 3-6 are groups of Cs and 1 is the breast tissue of the heterogeneous phantom of $40 \%$ glandularity; (b) noisefree2D mammographic image simulated at craniocaudal view, showing the breast tissue composition, the irregular lesion and the four groups of Cs. 
Table 6.6. Simple homogenous and complex heterogeneous breast phantom characteristics.

\begin{tabular}{llll}
\hline & Object & \multicolumn{1}{c}{ Type } & \multicolumn{1}{c}{ Dimensions } \\
\hline Simple breast & 1 & Breast tissue (50\%gland/50\%fat) & $\begin{array}{l}\text { [x,y,z] } 70 \times 70 \times 40 \mathrm{~mm}^{3} \\
\text { diameter } 1.0 \mathrm{~mm}\end{array}$ \\
& 2 & Group of 4 Cs (CaCo3) & diameter $6.0 \mathrm{~mm}$ \\
& 3 & Mass (water) & diameter $8.0 \mathrm{~mm}$ \\
CT based & 4 & Breast mass (75\%gland/25\%fat) & diameter $5.0 \mathrm{~mm}$ \\
breast & 5 & Breast mass (75\%gland/25\%fat) & diameter $3.0 \mathrm{~mm}$ \\
phantom & 6 & Breast mass (75\%gland/25\%fat) & \\
& 1 & Breast tissue (37\%gland 63\%fat) & 175 x $48 \times 134 \mathrm{~mm}$ \\
& 2 & Mass (water) & diameter $8.5 \mathrm{~mm}$ \\
& 3 & Group of 6 Cs (CaCo3) & diameter $1.3 \mathrm{~mm}$ \\
& 4 & Group of 6 Cs (CaCo3) & diameter $1.0 \mathrm{~mm}$ \\
& 5 & Group of 6 Cs (CaCo3) & diameter $0.4 \mathrm{~mm}$ \\
& 6 & Group of 5Cs (CaCo3) & diameter $0.2 \mathrm{~mm}$ \\
\hline
\end{tabular}

\section{B. Acquisition Simulation}

The acquisition geometry for the simulation of BT, shown in Fig. 6.21(a), was equivalent to that of the Siemens Mammomat Inspiration System. The acquisition scan included 25 equally spaced x-ray projection images simulated within a limited arc of $50^{\circ}$. Source-toisocenter distance (SID) was $603 \mathrm{~mm}$, while source to detector distance (SDD) was $650 \mathrm{~mm}$. Images with a size of $480 \times 480$ square pixels with a pitch of $170 \mu \mathrm{m}$ were simulated for the simple phantom and images of $800 \times 800$ square pixels with a pitch of $200 \mu \mathrm{m}$ were simulated for the complex heterogeneous phantom, respectively (Table 6.7). The pixel dimensions were chosen as a trade-off between computational time and resolution of objects on images that should be enough for performing image quality evaluation. In addition, images of selected ROIs from the simple homogeneous phantom were simulated with higher resolution, i.e. $50 \mu \mathrm{m}$. This pixel resolution was chosen as a realistic resolution that may be obtained at synchrotron facility. 
Table 6.7. Acquisition scenarios for both 2D mammography and BT. In case of BT, 25 projection images are acquired in the range $\left(-25^{\circ}: 25^{\circ}\right)$.

\begin{tabular}{|c|c|c|c|}
\hline Phantom & Beam Type & Photons per $\mathbf{m m}^{2}$ & Image size (pixels), pixel size \\
\hline \multirow[t]{8}{*}{ Simple phantom } & $28 \mathrm{kVp} \mathrm{W/Rh}$ & $8.10 \times 10^{5}$ & \multirow[b]{2}{*}{$480 \times 480,170 \mu \mathrm{m}$} \\
\hline & & $8.10 \times 10^{6}$ & \\
\hline & & $1.62 \times 10^{7}$ & \multirow[t]{2}{*}{$480 \times 480,50 \mu \mathrm{m}$} \\
\hline & & $2.43 \times 10^{7}$ & \\
\hline & $19 \mathrm{keV}$ & $8.10 \times 10^{5}$ & \multirow[b]{2}{*}{$480 \times 480,170 \mu \mathrm{m}$} \\
\hline & & $8.10 \times 10^{6}$ & \\
\hline & & $1.62 \times 10^{7}$ & \multirow[t]{2}{*}{$480 \times 480,50 \mu \mathrm{m}$} \\
\hline & & $2.43 \times 10^{7}$ & \\
\hline CT based & $28 \mathrm{kVp} \mathrm{W} / \mathrm{Rh}$ & $1.62 \times 10^{7}$ & \multirow[t]{2}{*}{$800 \times 800,200 \mu \mathrm{m}$} \\
\hline \multirow[t]{3}{*}{ breast phantom } & & $2.43 \times 10^{7}$ & \\
\hline & $19 \mathrm{keV}$ & $1.62 \times 10^{7}$ & \multirow[t]{2}{*}{$800 \times 800,200 \mu \mathrm{m}$} \\
\hline & & $2.43 \times 10^{7}$ & \\
\hline
\end{tabular}

$\mathrm{X}$-ray interactions in both breast models were simulated using an in-house developed Monte Carlo x-ray simulation software package (Bliznakova et al 2010b). This program has been reworked for voxel based phantoms and adjusted for mammography simulations (Bliznakova et al 2005, Bliznakova et al 2012b). The program followed the histories of single photons, emitted from the x-ray source as they pass through the breast phantom and then reach the detector. Photon transport is modelled based on the Monte Carlo method. The distance between two successive interactions is sampled, based on the relevant attenuation cross-sections, accounting for the different media along the photon path. For the used mammographic $x$-ray energy range, three interaction processes are considered, i.e. the photoelectric effect, the coherent and the incoherent scattering. Any time an interaction occurs, inside the phantom, one of the three interaction processes is selected by random sampling, according to the relative cross-sections of the processes at the specific photon energy and medium (Lazos et al 2003). The dose is calculated in Cartesian voxels as described previously. The energy deposited by photons and electrons in each voxel that contained gland tissue is accumulated and consequently is divided by the mass of this voxel. This results in a total glandular dose per voxel. The total MGD was computed by averaging all dose per voxel values over the entire glandular volume as described in Yi et al (Yi et al 2011). For the homogeneous case, each voxel of the breast volume that does not contain 
simulated abnormality, was comprised by a mixture of $50 \%$ adipose and $50 \%$ glandular weighted by mass. Based on that, the MGD was scored. To speed up the simulations, parallel execution on GPU was adapted. With this configuration, the time needed to produce a synthetic mammogram of the simple and complex phantoms for the highest photon fluence was 10 and 30 hours, respectively.

The synthetic mammograms simulated with this acquisition geometry, represent 2D spatial distribution of the energy of the x-rays that pass the breast model and reach the front part of the detector. The images are synthesized for a monochromatic beam of $19 \mathrm{keV}$ and for a common $28 \mathrm{kVp}$ tungsten $(\mathrm{W})$ target and $50 \mu \mathrm{m}$ rhodium (Rh) filter mammographic spectrum (Boone et al 1997, Boone 1998) with a mean energy of $18.8 \mathrm{keV}$ and a first $\mathrm{HVL}=0.454 \mathrm{~mm}$ Al. In both monochromatic and polychromatic cases, the total number of photons was kept the same and equal to $1.62 \times 10^{7}$ photons $/ \mathrm{mm}^{2}$. The spectrum representing the distribution of the number of photons per energy level is shown in Fig. 6.21(b). In the case of BT, the total number of photons used for the 2D mammogram was equally distributed in the 25 projection images. Additional levels of incident photon fluence were simulated as listed in Table 6.7. In the present $\mathrm{x}$-ray simulations, an ideal detector was assumed, while an anti-scatter grid and compression plates were not considered.

Entrance surface air kerma (ESAK) is calculated as the photon fluence after the filter (photons $/ \mathrm{mm}^{2}$ ) over the photon fluence per $\mathrm{mR}$ in air and then converted to $\mathrm{mGy}$.

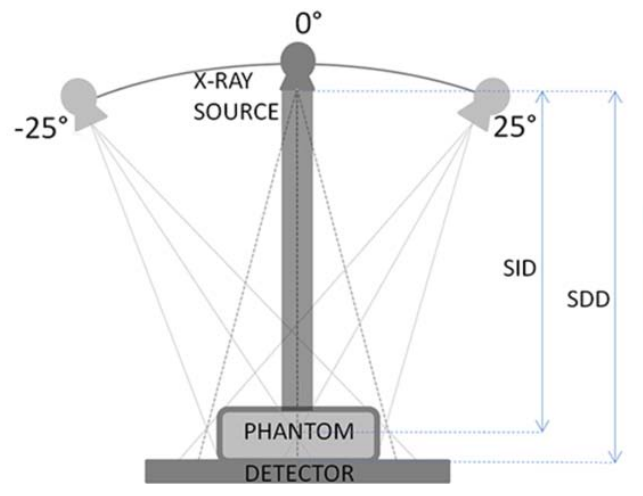

(a)

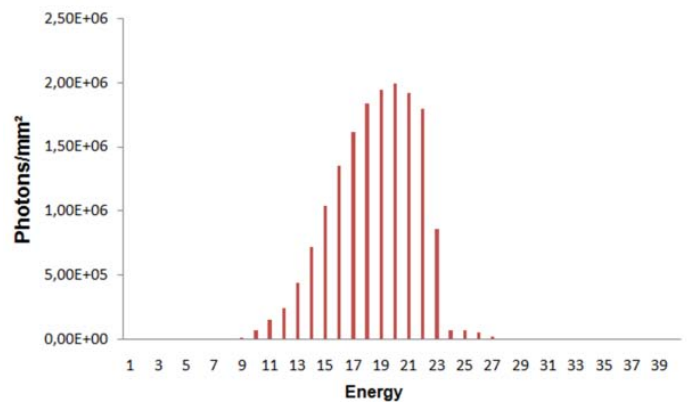

(b)

Fig. 6.21. Simulated BT and 2D mammography acquisition geometry: (a) schematic representation of the acquisition system (b) simulated $28 \mathrm{kVp}$ W/Rh mammographic spectrum.

In addition to estimating the MGD from our Monte Carlo simulations, we also estimated MGD for all simulated image acquisitions using previously published models. For monochromatic beams, the MGD was calculated as MGD=ESAK $\mathrm{x} g$, where $\mathrm{g}$ is the incident air kerma to MGD conversion factor from Boone et al (Boone 2002). For polychromatic beam, the MGD was calculated as MGD=ESAK x $\mathrm{g} \times \mathrm{c} \times \mathrm{s}$ from Dance et al (Dance et al 2000a). In this model, the factor c corrects for any difference from $50 \%$ glandularity in breast 
composition, and s corrects for any difference from the original tabulation by Dance (Dance 1990) due to the use of a different $x$-ray spectrum factor. Specifically, the c value for $40 \%$ glandular tissue was estimated by interpolation between the g values provided for $25 \%$ gland and $50 \%$ gland and the s factor was set to 1.042 for the W/Rh spectrum.

A simple backprojection method was used as a tomosynthesis reconstruction algorithm. Projection filtering prior to the reconstruction was not applied. Tomosynthesis slices were separated by $1 \mathrm{~mm}$.

\section{Evaluation}

The variation of several image characteristics: CNR, C and FOM were calculated. For the homogeneous phantom, the average value $\bar{\mu}_{f}$ was calculated over a circular ROI inside each feature with a diameter of 35 pixels (5.8mm), 25 pixels $(4.2 \mathrm{~mm})$ and 4 pixels $(0.7 \mathrm{~mm})$ for the water insert (6mm diameter), the glandular mass $(5 \mathrm{~mm})$ and the Cs $(1 \mathrm{~mm})$, respectively. The properties of the background ( $\bar{\mu}_{b k}$ and $\sigma_{b k}$ ) were evaluated for a square area of $60 \times 60$ pixels $\left(10 \times 10 \mathrm{~mm}^{2}\right)$ located between the water insert and the two bigger glandular masses. In the case of the heterogeneous phantom, the background ROI analyzed was a rectangular $30 \mathrm{x} 40 \mathrm{~mm}^{2}$ area of breast tissue located in the space between the chest wall and the groups of Cs. For the water insert simulating an irregular mass $(8.5 \mathrm{~mm})$, a circular ROI of $5.5 \mathrm{~mm}$ diameter inside the feature was analyzed and the mean value of the pixels was calculated as $\bar{\mu}_{f}$. Similarly, for the Cs, circular ROIs inside each feature were defined. For Cs belonging to the two bigger groups $(1.3 \mathrm{~mm}$ and $1 \mathrm{~mm}), \bar{\mu}_{f}$ for each feature was calculated from the mean value of the pixels inside ROIs of diameters $0.8 \mathrm{~mm}$ and $0.6 \mathrm{~mm}$ respectively. For Cs belonging to the two smaller groups, areas that contained each feature were defined and the maximum value was extracted and used as $\mu_{f}$ in each case.

\subsubsection{Results}

\section{A. Dose evaluation}

Table 6.8 summarizes the ESAK values and the mean glandular dose estimations using the previously published models and our own Monte Carlo (MC) simulations. 
Table 6.8. ESAK and MGD dose calculations

\begin{tabular}{|c|c|c|c|c|c|c|}
\hline Phantom & $\begin{array}{l}\text { Beam } \\
\text { Type }\end{array}$ & $\begin{array}{c}\text { Incident } \\
\text { photon } \\
\text { fluence(Photon } \\
\text { sper } \mathbf{~ m m}^{2} \text { ) }\end{array}$ & $\begin{array}{l}\text { ESAK } \\
(\mathrm{mGy})\end{array}$ & $\begin{array}{l}\text { MGD } \\
\text { (mGy) } \\
\text { comparison } \\
\text { estimations }\end{array}$ & $\begin{array}{c}\text { MGD }_{M C} \\
\text { (mGy) } \\
\text { MC } \\
2 D^{b}\end{array}$ & $\begin{array}{c}\text { MGD }_{M C} \\
(\mathrm{mGy}) \\
\text { MC } \\
\text { BT }^{\mathbf{b}}\end{array}$ \\
\hline Simple & 28 & $8.10 \times 10^{5}$ & 0.1828 & 0.06 & 0.05 & 0.05 \\
\hline \multirow[t]{7}{*}{ phantom } & $\mathrm{kVp}$ & $8.10 \times 10^{6}$ & 1.8286 & 0.56 & 0.53 & 0.52 \\
\hline & W/Rh & $1.62 \times 10^{7}$ & 3.6571 & 1.11 & 1.05 & 1.04 \\
\hline & & $2.43 \times 10^{7}$ & 5.4857 & 1.67 & 1.58 & 1.57 \\
\hline & 19 & $8.10 \times 10^{5}$ & 0.1564 & 0.06 & 0.05 & 0.05 \\
\hline & $\mathrm{keV}$ & $8.10 \times 10^{6}$ & 1.5643 & 0.57 & 0.55 & 0.54 \\
\hline & & $1.62 \times 10^{7}$ & 3.1285 & 1.13 & 1.10 & 1.09 \\
\hline & & $2.43 \times 10^{7}$ & 4.6928 & 1.70 & 1.65 & 1.64 \\
\hline CT breast & 28 & $1.62 \times 10^{7}$ & 3.6571 & 1.16 & 1.29 & 1.27 \\
\hline \multirow[t]{4}{*}{ phantom } & $\mathrm{kVp}$ & $2.43 \times 10^{7}$ & 5.4857 & 1.73 & 1.93 & 1.91 \\
\hline & W/Rh & & & & & \\
\hline & 19 & $1.62 \times 10^{7}$ & 3.1285 & 1.17 & 1.35 & 1.34 \\
\hline & $\mathrm{keV}$ & $2.43 \times 10^{7}$ & 4.6928 & 1.76 & 2.03 & 2.00 \\
\hline
\end{tabular}

aa(Boone J M 2002, Dance D R 1990, Dance D R et al 2000)

${ }^{\mathrm{b}}$ Current study

Our Monte Carlo estimations were close to the calculations using the Dance and Boone models (Table 6.8). Dose calculations revealed that for equal exposure, ESAK was higher for polychromatic case by $17 \%$ compared to monochromatic, while the MGD values were very similar.

\section{B. Polychromatic versus monochromatic beams for equal incident photon fluence}

i. Simple phantom.

A comparison of 2D mammographic images and BT slices obtained with monochromatic 19 $\mathrm{keV}$ and polychromatic $28 \mathrm{kVp}$ x-ray beams is shown in Fig. 6.22. These images have been acquired using the simple phantom with photon fluence of $1.62 \times 10^{7}$ photons $/ \mathrm{mm}^{2}$ and detector pixel size equal to $170 \times 170 \mu \mathrm{m}^{2}$. Figure 6.22 (a) shows the comparison of $2 \mathrm{D}$ projection images and tomosynthesis slices obtained with monochromatic $19 \mathrm{keV}$ and polychromatic 28 $\mathrm{kVp}$ beams while the corresponding intensity profiles taken across the $6 \mathrm{~mm}$ water mass placed in the middle of the phantom are shown in Fig. 6.22(b). 


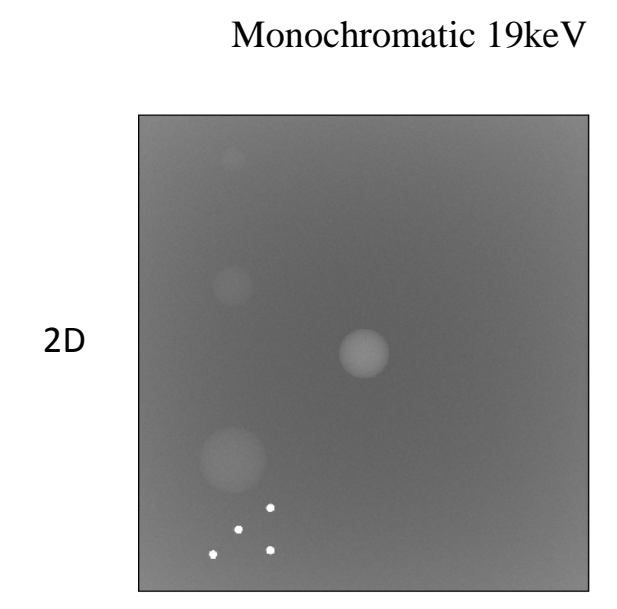

Polychromatic $28 \mathrm{kVp}$
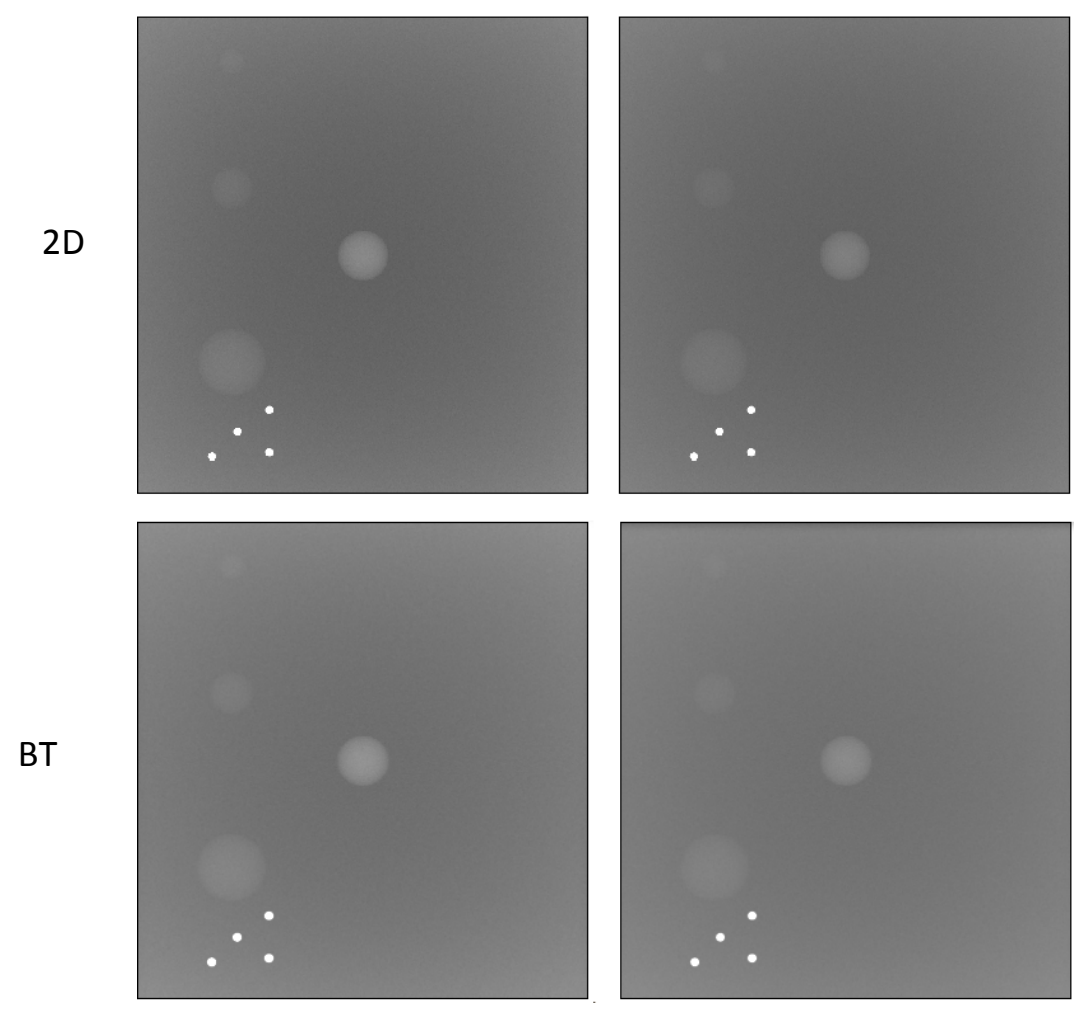

(a)
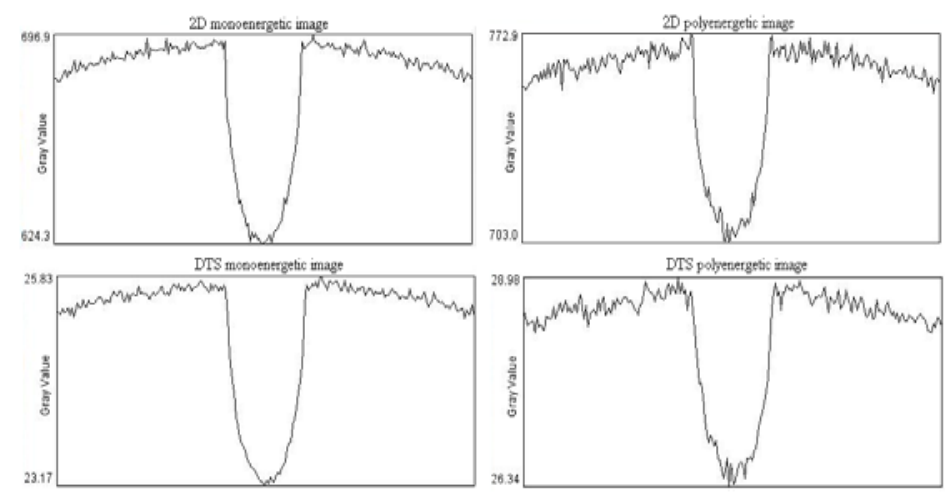

(b)

Fig. 6.22. (a) Simulated monochromatic $19 \mathrm{keV}$ (left) and polychromatic $28 \mathrm{kVp}$ (right) images of the simple phantom: 2D mammography image (upper row) and BT slice (lower row) for photon fluence of $1.62 \times 10^{7}$ photons $/ \mathrm{mm}^{2}$. Detector pixel size was170 $\mu \mathrm{m}$ and (b) the corresponding intensity profiles of the water mass. 
Regions of interest from intensity 2D projection images with monochromatic $19 \mathrm{keV}$ and polychromatic $28 \mathrm{kVp}$ obtained for a detector pixel size of $50 \mu \mathrm{m}$ and the same photon fluence of $1.62 \times 10^{7}$ photons $/ \mathrm{mm}^{2}$ are shown in Fig. 6.23.

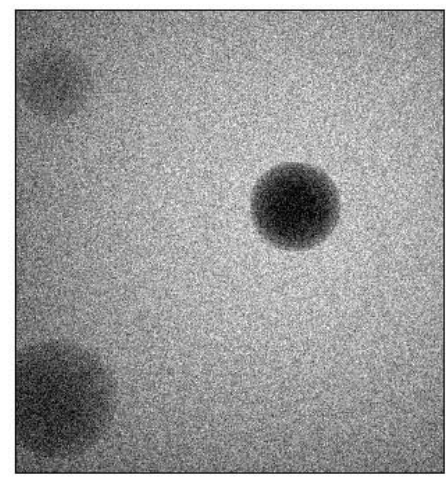

(a)

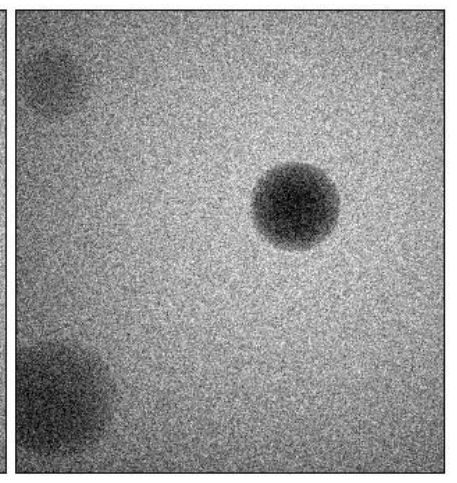

(b)

Fig. 6.23. ROIs from simulated 2D projection images of the simple phantom obtained with monochromatic $19 \mathrm{keV}$ (left) and polychromatic $28 \mathrm{kVp}$ (right) beams, for photon fluence of $1.62 \times 10^{7}$ photons $/ \mathrm{mm}^{2}$. Detector pixel size was $50 \mu \mathrm{m}$.

It can be observed from Figs.6.22 and 6.23 that images obtained with monochromatic and polychromatic beams are similar in their visual appearance. However, in case of monochromatic beam, the breast masses are characterized with improved edge detection and visualization for both mammography and BT. These large low-contrast features have attenuation coefficients close to that of the breast tissue, which makes it harder to distinguish them from the background compared to the group of Cs.

Further, features in 2D images and tomosynthesis slices were evaluated quantitatively by calculating CNR, C and FOM for both monochromatic and polychromatic x-ray beams. Figures 6.24(a)-6.24(c) show the corresponding CNR, C and FOM graphs of the spherical water insert $(6 \mathrm{~mm})$, glandular mass $(5 \mathrm{~mm})$ and group of Cs $(1 \mathrm{~mm})$ visible on the 2D images and BT slices. For the case of Cs, the values that are presented correspond to the average values of the four Cs. The quantitative results presented are for images acquired with $1.62 \times 10^{7}$ photons $/ \mathrm{mm}^{2}$ and pixel size of $170 \mu \mathrm{m}$. 
water insert
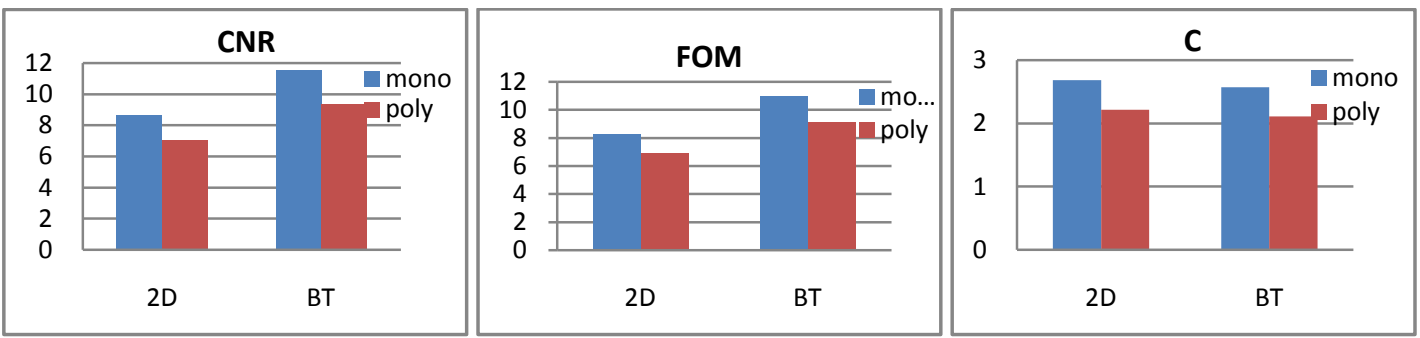

(a)

glandular mass
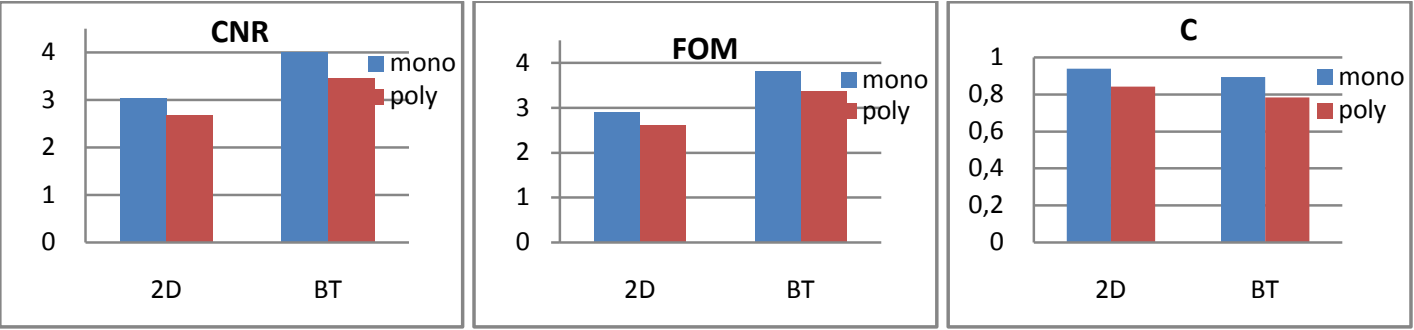

(b)

\section{group of Cs}
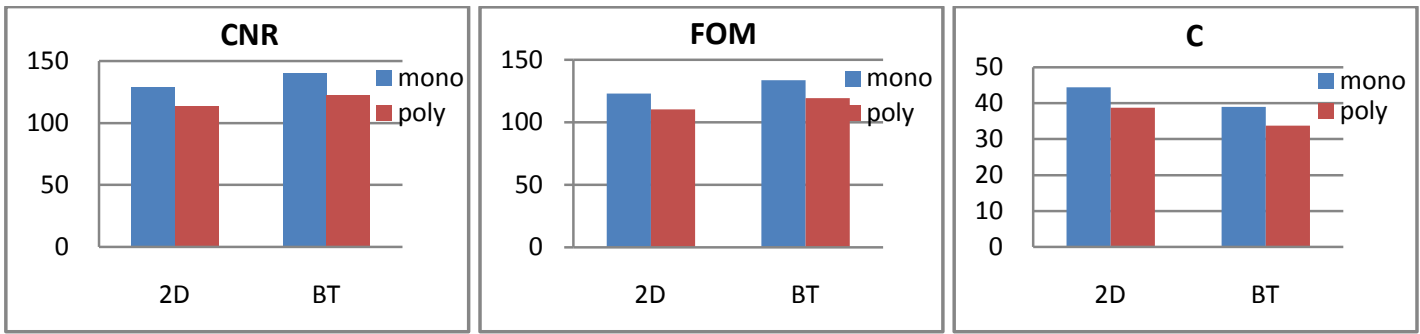

(c)

Fig. 6.24. Evaluation performed on 2D and BT images of the simple phantom acquired with monochromatic and polychromatic beam (photon fluence of $1.62 \times 10^{7}$ photons $/ \mathrm{mm}^{2}$ and pixel size of $170 \mu \mathrm{m}$ ). CNR, FOM and C graphs for (a) water insert with size of $6 \mathrm{~mm}$, (b) glandular mass with size of $5 \mathrm{~mm}$ and (c) Cs with size of $1 \mathrm{~mm}$.

The quantitative analysis shows that monochromatic beam results in higher image quality compared to polychromatic for all figures of merit (CNR, C and FOM) confirming the visual observations. The percentage of the CNR improvement of monochromatic over polychromatic is similar for both 2D and BT sets (slightly higher in case of BT) and varied between 14\%-23\% with the highest benefit observed for the water mass and less improvement for the Cs group. The corresponding FOM values were increased by $12 \%$ for the group of Cs, $13 \%$ for the glandular mass and $20 \%$ for the water insert, while the improvement in C values was higher and ranged between $15 \%-22 \%$.

The comparison between BT and 2D imaging showed lower $\mathrm{C}$ values for BT compared to 2D case. However, BT performed with monochromatic beam, has comparable or higher (in case of the masses) $\mathrm{C}$ values than polychromatic 2D conventional mammography. 


\section{ii. CT breast phantom}

Similarly to the simple breast phantom case, Fig. 6.25 shows the 2D and the tomosynthesis slices for the heterogeneous CT-based breast phantom obtained with simulation of monochromatic and polychromatic beams with a photon fluence of $1.62 \times 10^{7}$ photons $/ \mathrm{mm}^{2}$ and detector pixel size equal to $200 \mu \mathrm{m}$.

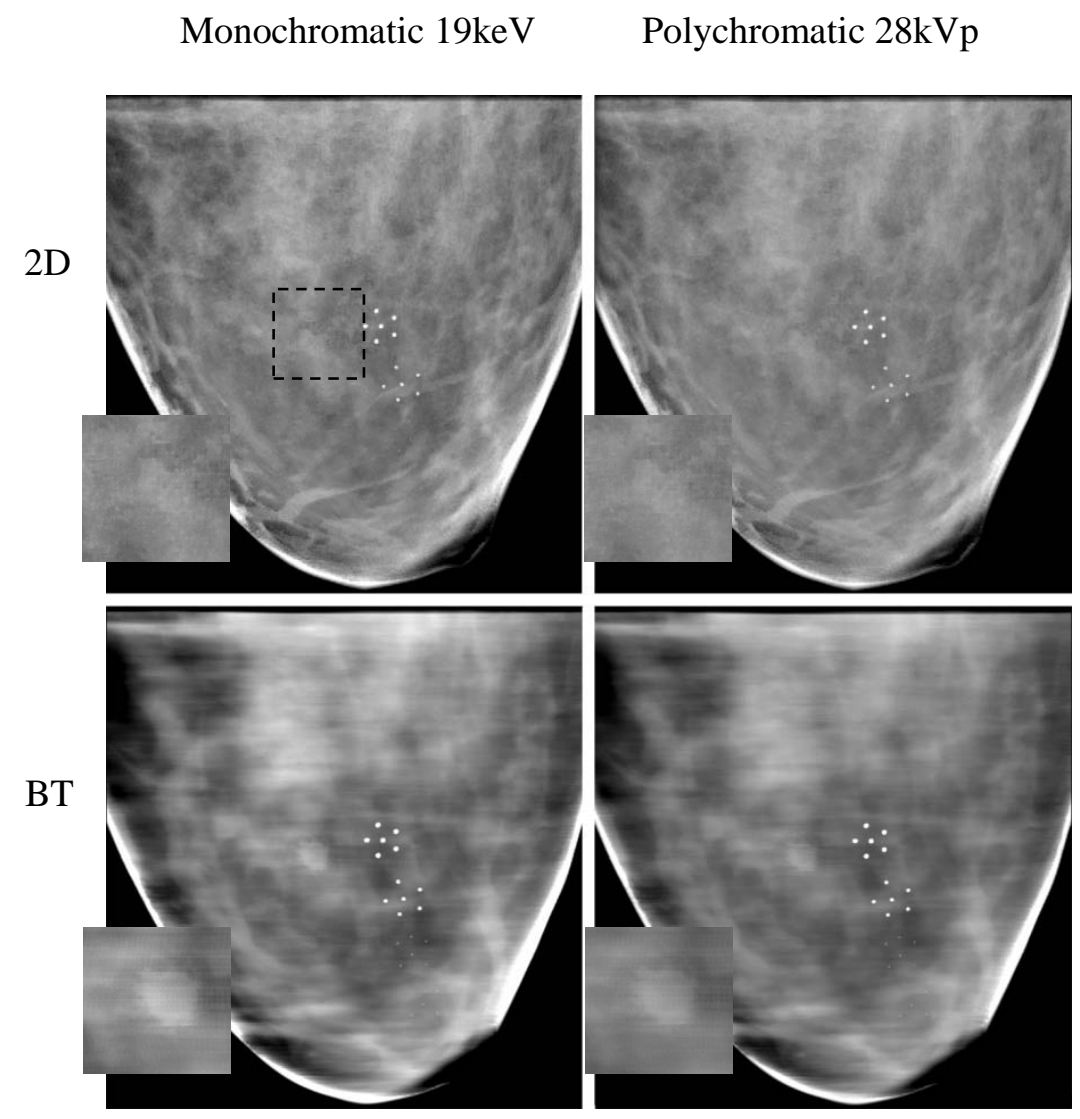

Fig. 6.25. Monochromatic $19 \mathrm{keV}$ (left) and polychromatic $28 \mathrm{kVp}$ (right) images: 2D (upper row), BT (lower row) of the complex breast phantom for photon fluence of $1.62 \times 10^{7}$ photons $/ \mathrm{mm}^{2}$ and detector pixel size of $200 \mu \mathrm{m}$. In the inserts of the 2D and BT images, ROIs that contain the irregular mass are presented.

It can be observed that the visibility of the features of interest, and especially of the lowcontrast mass, is much improved in the BT images compared to the $2 \mathrm{D}$ in the case of the heterogeneous breast phantom. Overlying breast anatomy in 2D images hides the borders of the mass and makes it difficult to detect its irregular shape which is very important for the characterization of the lesions. Moreover, acquisition with the monochromatic beam resulted in increased contrast and thus better detection and visualization of all features.

Figures 6.26(a) and 6.26(b) depict the quantitative evaluation for 2D and BT images with monochromatic and polychromatic x-ray beams. The features that were evaluated are the irregular mass with size of $8.5 \mathrm{~mm}$ and the four groups of Cs. For the case of Cs, the values presented correspond to the average values of the four groups Cs (1.3, 1, 0.4 and $0.2 \mathrm{~mm}$ ). The results are from images acquired with $1.62 \times 10^{7}$ photons $/ \mathrm{mm}^{2}$ and $200 \mu \mathrm{m}$ resolution. 


\section{water insert}

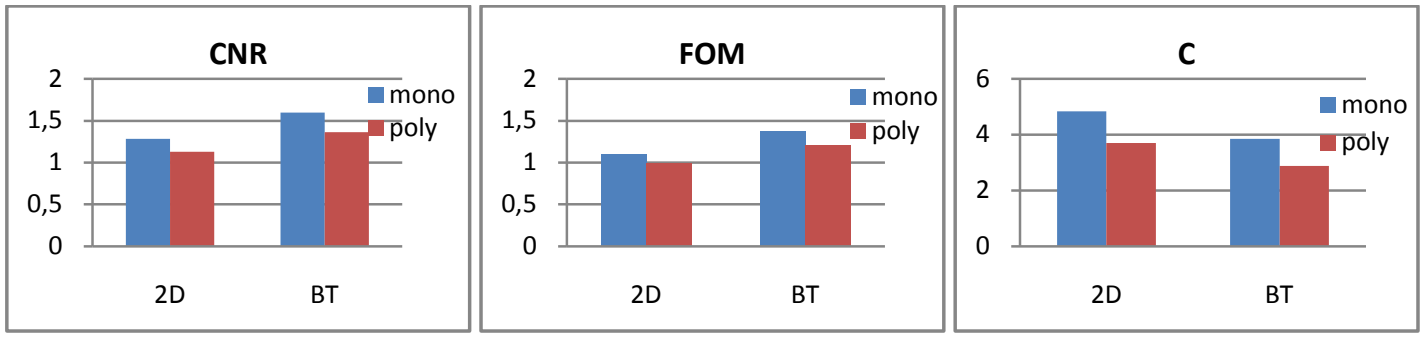

(a)

group of Cs
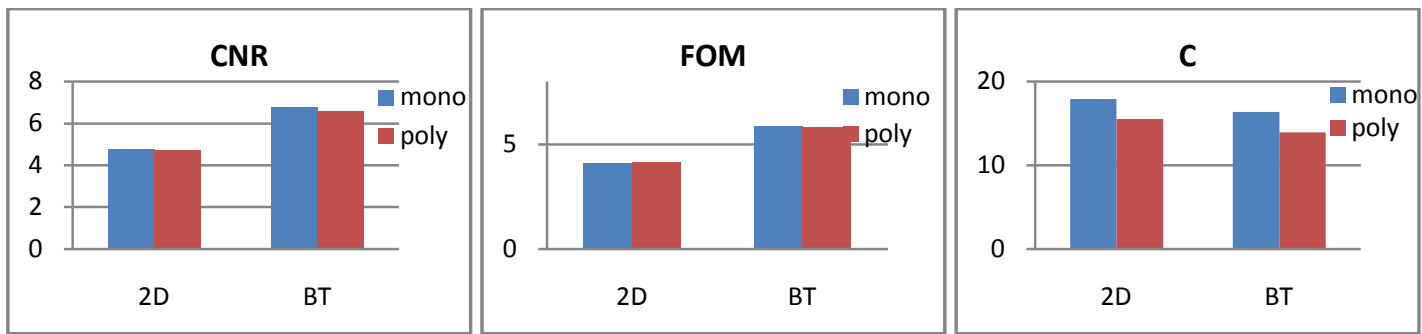

(b)

Fig. 6.26. Evaluation performed on 2D and BT images of the heterogeneous CT-based breast phantom simulated with monochromatic and polychromatic beam (photon fluence of $1.62 \times 10^{7}$ photons $/ \mathrm{mm}^{2}$ and pixel size of $200 \mu \mathrm{m})$. CNR, FOM and C graphs for (a) irregular mass with size of 8.5mm and (b) 4 groups of Cs.

The benefit of BT in case of breasts with heterogeneous background can be seen in the results shown in Fig. 6.26. Apart from the visual improvement that was observed in the images, quantitative analysis confirms the improvement with an increase in CNR values for the case of water insert and groups of Cs. Unlike CNR, the C values are reduced in BT compared to 2D, but still monochromatic $\mathrm{BT}$ is better than polychromatic $2 \mathrm{D}$, similarly to the case of simple homogeneous breast phantom.

Although the use of a monochromatic beam improves image quality, in the case of heterogeneous phantoms, both the lesions and the anatomical structure of the background are enhanced, the latter increasing the standard deviation of the background. This can explain the lower CNR and FOM improvement of monochromatic images compared to the case of simple phantom (homogeneous background), despite the improved visibility of the features of interest in both cases. Thus, for the case of the breast phantom with realistic tissue distribution, the use of monochromatic beam demonstrates benefits mainly in C. An increase of $33.4 \%$ and $17 \%$ is achieved from the monochromatic BT compared to the polychromatic $\mathrm{BT}$ for the low- and high-contrast features, respectively. The corresponding $\mathrm{C}$ improvement between monochromatic and polychromatic acquisitions in the 2D case is similar, i.e. $30.7 \%$ and $15 \%$. 


\section{Low dose tomosynthesis. Simulation studies with different ESAK.}

Initial results suggest that the improved image quality of using a monochromatic beam could allow for acquisitions with lower doses. Visual comparison showed that in all cases, features were better visualized in monochromatic images compared to the polychromatic ones of the same photon fluence as in the case demonstrated in Fig. 6.27 for $1.62 \times 10^{7}$ photons $/ \mathrm{mm}^{2}$.

As can be seen in Fig.6.27, CNR improves with the increase in the MGD as expected, while the $\mathrm{C}$ was found to be almost constant (data not shown due to the very close values for the five doses evaluated and not affected with the dose increase).

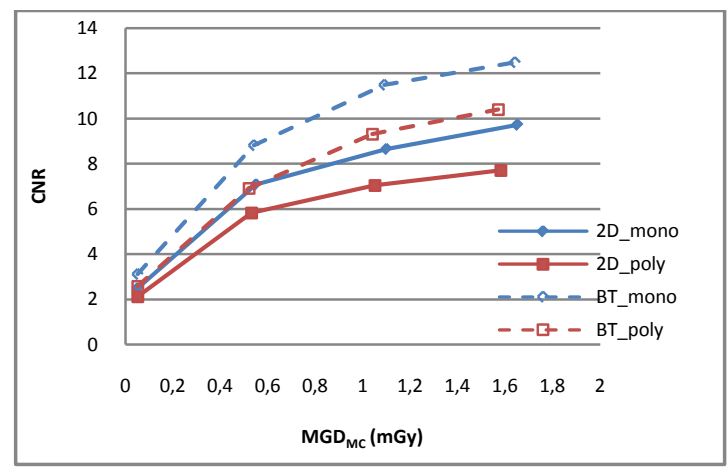

(a)

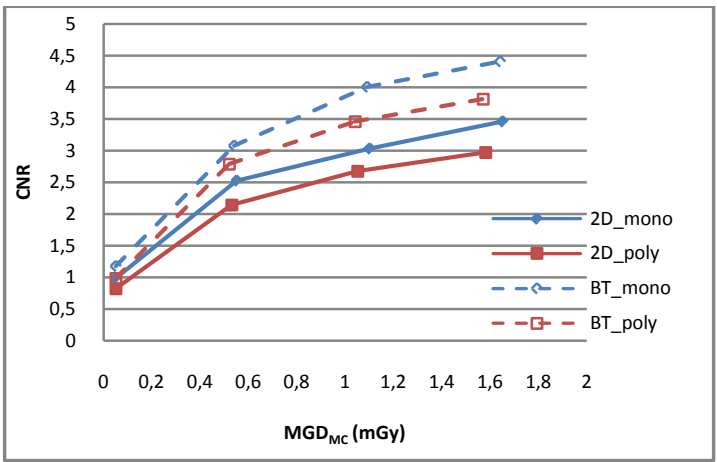

(b)

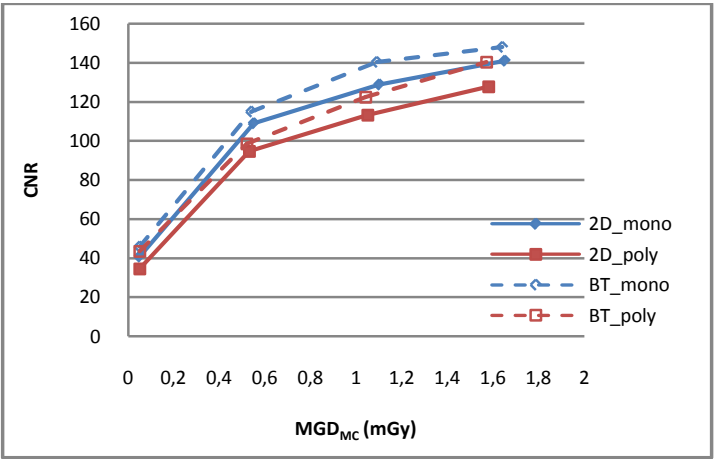

(c)

Fig. 6.27. Evaluation of $\mathrm{CNR}$ for different $\mathrm{MGD}_{\mathrm{MC}}$ levels for $2 \mathrm{D}$ and $\mathrm{BT}$ images with monochromatic and polychromatic x-ray beams for the simple phantom (a) water insert, (b) glandular mass and (c) group of Cs. 
The CNR-dose graphs show that by using monochromatic beam, we can achieve CNR values comparable to polychromatic acquisition but with lower MGD (and ESAK). Specifically, comparable CNR values for the case of breast masses and Cs can be obtained when using monochromatic beam with an incident exposure that results in a MGD that is 1.5 times lower than when using a polychromatic beam.

The above quantitative results are well demonstrated in Fig.6.28. Specifically, Figs. 6.28(a) and 6.28(b) show the monochromatic and polychromatic BT slices corresponding to equal incident photon fluence of $2.43 \times 10^{7}$ photons $/ \mathrm{mm}^{2}$ with the former showing advantage in feature visualization and image quality, as discussed in section 3.2.1 (for equal photon fluence of $1.62 \times 10^{7}$ photons $/ \mathrm{mm}^{2}$ ), while Fig. 6.28(c) is the monochromatic BT slice acquired with the lower incident photon fluence of $1.62 \times 10^{7}$ photons $/ \mathrm{mm}^{2}$. This image has comparable noise characteristics to the polychromatic one and also an increase of $15-22 \%$ in C for the different features evaluated, achieved with MGD that is 1.5 times lower than the MGD obtained with the polychromatic beam.

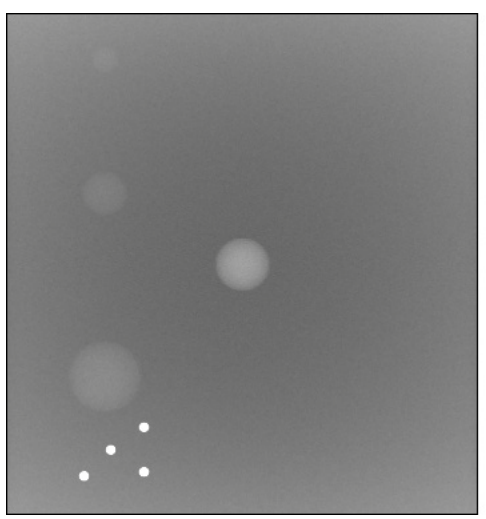

(a)

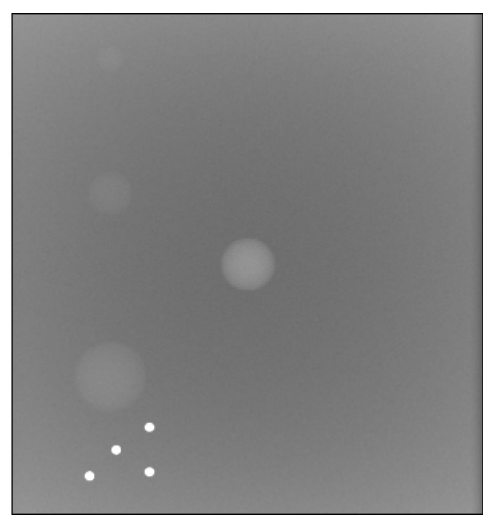

(b)

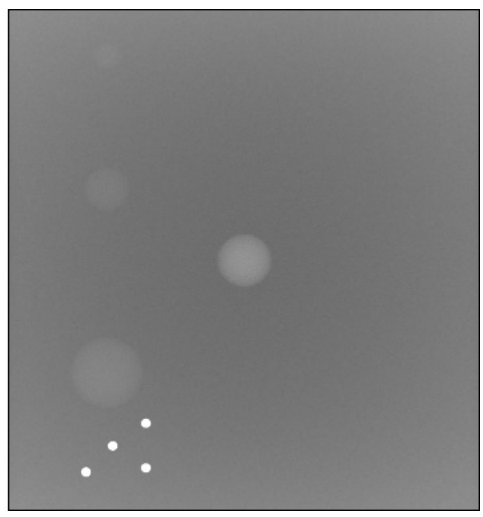

(c)

Fig. 6.28. BT images showing the simple homogeneous breast phantom: (a) monochromatic image, photon fluence $=2.43 \times 10^{7}$ photons $/ \mathrm{mm}^{2}, \quad M G D_{\mathrm{MC}}=1.64 \mathrm{mGy}$, (b) polychromatic image, photon fluence $=2.43 \times 10^{7}$ photons $/ \mathrm{mm}^{2}, \quad \mathrm{MGD}_{\mathrm{MC}}=1.57 \mathrm{mGy}$ and(c) monochromatic image, photon fluence $=1.62 \times 10^{7}$ photons $/ \mathrm{mm}^{2}, \mathrm{MGD}_{\mathrm{MC}}=1.09 \mathrm{mGy}$. Detector pixel size was $170 \mu \mathrm{m}$.

Similarly, images acquired using these two different exposures for the complex phantom, are presented. Figures 6.29(a) and 6.29(b) show the polychromatic 2D and BT images corresponding to $2.43 \times 10^{7}$ photons $/ \mathrm{mm}^{2}$, while Fig. 6.29 (c) is the monochromatic BT image simulated with lower incident photon fluence $\left(1.62 \times 10^{7}\right.$ photons $\left./ \mathrm{mm}^{2}\right)$ that resulted in lower (1.5 times) MGD. 


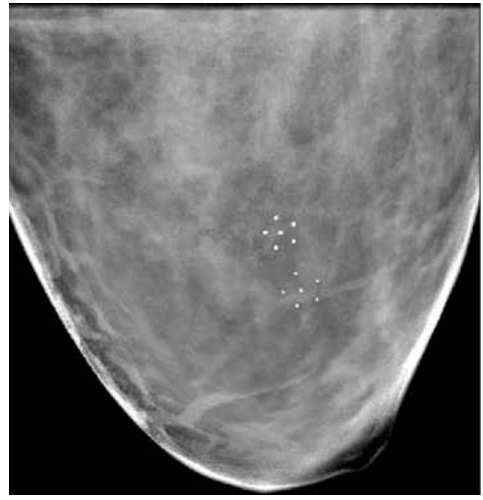

(a)

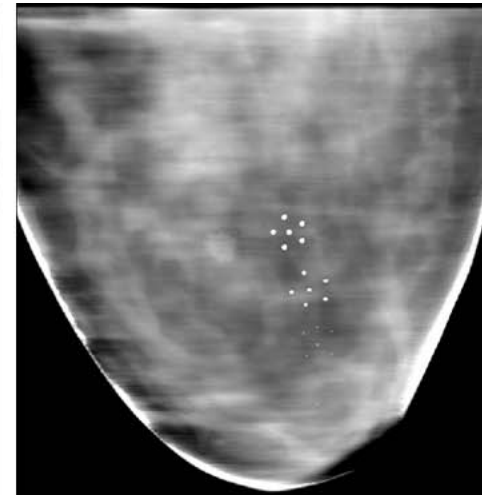

(b)

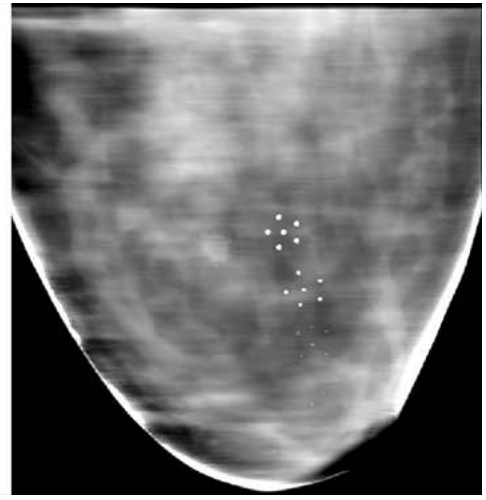

(c)

Fig. 6.29. Simulated images showing the heterogeneous CT-based breast phantom: (a) polychromatic 2D image, photon fluence $=2.43 \times 10^{7}$ photons $/ \mathrm{mm}^{2}, \mathrm{MGD}_{\mathrm{MC}}=1.93 \mathrm{mGy}$, (b) polychromatic BT image, photon fluence $=2.43 \times 10^{7}$ photons $/ \mathrm{mm}^{2}, \mathrm{MGD}_{\mathrm{MC}}=1.91 \mathrm{mGy}$ and(c) monochromatic BT image, photon fluence $=1.62 \times 10^{7}$ photons $/ \mathrm{mm}^{2}, \mathrm{MGD}_{\mathrm{MC}}=1.34 \mathrm{mGy}$. Pixel size was $200 \mu \mathrm{m}$.

The comparison between the polychromatic BT and the monochromatic BT (Figs. 6.29(b) and 6.29(c)) shows comparable noise characteristics and a C improvement of 33\% and 17\% for the mass and the Cs, respectively for the case of monochromatic BT with an incident exposure that results in a lower (1.5 times) MGD compared to the polychromatic BT.

\subsubsection{Conclusions}

In this study the impact on image quality of using monochromatic beams for BT imaging was investigated. Results indicate that monochromatic beams could enable a reduction of dose in tomosynthesis without compromising image quality in terms of CNR or could even result in an increase in C. Monochromatic images can provide better detail and tissue differentiation and in combination with BT can lead to improvement in mass detection and visibility of borders, which is important for the characterization of the masses, especially when they are spiculated. Overall, monochromatic BT results in improved image quality compared to conventional mammography (polychromatic 2D) in most features evaluated, in addition to providing useful depth information. The results obtained in these simulations are encouraging for the development of a tomosynthesis system based on monochromatic beams. 
CHAPTER 7

CONCLUSIONS AND FUTURE WORK DIRECTIONS

\section{CHAPTER SUMMARY}

Chapter 7 summarizes the results and conclusion remarks of the present thesis and points out future research direction in this field. 
Breast tomosynthesis imaging techniques with monochromatic radiation are investigated using a computer-based platform incorporating MPA and BP reconstruction algorithms combined with several filters. A GUI developed for the BT platform enables easy and fast import of acquisition and image parameters, selection of the reconstruction algorithm and filtering methods and facilitates further the research.

Evaluation performed using initial simulations with software breast phantoms as well as real projections acquired with complex breast phantoms at a clinical setting, indicated similar performance for BP and MPA algorithms with the latter allowing a multifold reduction in reconstruction time. Both visual and quantitative assessment indicated close performance for the two algorithms with no considerable differences in feature detection, size and appearance of the background tissue. Results demonstrated that MPA is a time efficient algorithm, fully compliant with BT clinical systems. The same study, revealed also that features' location within phantoms of same thickness influence slightly the quality of the reconstruction in tomosynthesis slices. Compared to 2D mammography, BT shows advantage in visualizing features of small size and for increased phantom thickness or features within a dense heterogeneous background with superimposed structures.

Acquisition parameters such as the arc length and the number of projections were investigated in a simulation study using a homogeneous software breast phantom. The CNR for the studied mass revealed that it tends to increase with extending the arc length. An opposite behavior was observed for the high-contrast features. The evaluation for the different acquisition schemes indicated that the number of projections for each arc length is as important as optimizing the acquisition arc. In case of calcifications, where different feature sizes were evaluated, the optimal step increment that defines the number of projections per arc tends to increase as the size of feature to be identified decreases. Generally, it was found that maximizing or minimizing the number of projections in each arc, does not necessarily bring the same result on CNR.

The experimental study performed at Elettra Synchrotron facilities, in order to evaluate the image quality of breast-like lesions using monochromatic BT, indicated that the $6 \mathrm{~mm}$ lowcontrast features are better visualized at extended arc length confirming the results of the simulations. Among the studied acquisition geometries, the acquisition arc of $56^{\circ}$ with 15 projection images indicated the highest image reconstruction quality for the low-contrast mass based on the results from all figures of merit. The image quality of $\mu \mathrm{Cs}$ is less sensitive to the acquisition arc. Calcifications with size ranging between $170 \mu \mathrm{m}-500 \mu \mathrm{m}$, such as the presently studied high-contrast features, are well identified and visualized in all studied arcs. Moreover, a clear advantage of BT was demonstrated in bringing into focus and localizing 
low-contrast features hidden in a highly heterogeneous background. This study involved also optimization of the filtering method prior to the reconstruction and showed that using optimized filters in respect to the type of feature characteristics is important. Pre-filtering of projection images results in features with sharper edges and images with better quality. The anatomical background was suppressed and the features especially those of small size, were much better visualized. Similar conclusions for the filters were derived (were in agreement with) from all simulation/experimental studies performed. Among the studied reconstruction techniques, FMPA-MSINC showed a much better performance in all cases, enabling the reconstruction of both high- and low- contrast features with suitable adjustment of the filter's parameter.

The use of monochromatic beams has the potential to improve the quality of tomographic images with features having sharper edges and higher contrast at lower incident exposures. The results from the experimental study between images obtained from monochromatic and polychromatic beams showed that the use of monochromatic beams can produce images with higher CNR and C, acquired with half incident exposure. This fact could be used in a future tomosynthesis system based on monochromatic beams that may result in tomographic images of higher contrast at lower incident exposures.

In addition, 2D images failed to visualize low-contrast masses in the breast phantom. In contrary this low-contrast feature is visualized on BT images acquired with monochromatic beams at extended arc (i.e. $>40^{\circ}$ ) even when simple (non-filtered) MPA is used. Image quality and evaluation metrics were improved further when dual-energy techniques were applied along with monochromatic BT.

Simulation results indicated as well, that monochromatic beams could enable a reduction of dose in tomosynthesis without compromising image quality in terms of CNR and even result in an increase Contrast. Monochromatic images can provide better detail and tissue differentiation and in combination with BT can lead to improvement in mass detection and visibility of borders, which is important for the characterization of the masses, especially when they are spiculated, as the one evaluated in this feasibility study. Overall, monochromatic BT results in improved image quality compared to conventional mammography (polychromatic 2D) in most features evaluated, in addition of providing useful depth information. The results obtained in these simulations are encouraging for the development of a tomosynthesis system based on monochromatic beams. Reducing the radiation dose required for BT acquisition with no loss or even improvement of image quality is always desirable and it has been demonstrated that one way to obtain this is through the use of monochromatic beams with BT. The comparison performed in the current project was 
between the two types of beam: monochromatic and polychromatic under the same conditions. In order to evaluate the potential benefits of using monochromatic beams in BT, in both simulation and experimental studies, during the comparison of the two beams, the detectors, phantoms, mean beam energies and rest parameters were kept the same.

Future work could include Practitioner's subjective evaluation of acquired image quality using the two modalities, reduction of required scanning time and estimation of potential benefits of monochromaticity in terms of classification of lesions to benign/malignant. 
Two-Dimensional

3D

Three-Dimensional

$\mu \mathrm{Cs}$

microcalcifications

ACR

American College of Radiology

ASF

Artifact Spread Function

ART

Algebraic reconstruction techniques

APS

Advanced photon source

BIRADS

Breast Imaging Reporting and Data System

BP

Back Projection

BT

Breast Tomosynthesis

C

Contrast

CIRS

Computerized Imaging Reference Systems

CNR

Contrast to Noise Ratio

Cs

calcifications

CT

Computed Tomography

$\mathrm{DE}$

Dual energy

DE-BT

Dual energy breast tomosynthesis

DTS

Digital Tomosynthesis

ESAK

Entrance Surface Air Kerma

ESE

Entrance Surface Exposure

ESRF

European Synchrotron Radiation Facility

FOM

Figure of Merit

FTS

Flashing Tomosynthesis

GUI

Graphical User Interface

HVL

Half Value Layer

ILST

Iterative least squares technique

$\mathrm{MC}$

Monte Carlo

MGD

Mean Glandular Dose

MPA

Multiple Projection Algorithm

NAD

Nonlinear anisotropic diffusion

PMMA

Polymethyl methacrylate 
PSI

$\mathrm{RF}$

ROI

SAA

SID

SIRT

SDD

SNR

SR

SYRMEP

TACT
Paul Scherrer Institute

radio frequency

Region of Interest

Shift and Add

Source-to-isocenter distance

simultaneous iterative reconstruction technique

Source-to-detector distance

Signal to Noise Ratio

Synchrotron radiation

SYnchrotron Radiation for MEdical Physics

Tuned aperture computed tomography 


\section{BIBLIOGRAPHY}

Abrami A, Arfelli F, Barroso R C, Bergamaschi A, Bille F, Bregant P, Brizzi F, Casarin K, Castelli E, Chenda V, Palma L D, Dreossi D, Fava A, Longo R, Mancini L, Menk R H, Montanari F, Olivo A, Pani S, Pillon A, Quai E, Kaiser S R, Rigon L, Rokvic T, Tonutti M, Tromba G, Vaseotto A, Venanzi C, Zanconati F, Zanetti A \& Zanini F 2005 Medical applications of synchrotron radiation at the SYRMEP beamline of ELETTRA Nuclear Instruments \& Methods in Physics Research Section aAccelerators Spectrometers Detectors and Associated Equipment 548 221-227

American College of Radiology (ACR) Breast Imaging Reporting and Data System (BIRADS), 3rd ed. 1998 Reston, VA, American College of Radiology

Adachi S, Hirasawa S, Takahashi M, Okada H, Yamane Y \& Yamada S 2002 Noise properties of a Se-based flat-panel x-ray detector with CMOS readout integrated circuits Proc. SPIE, Medical Imaging 2002: Physics of Medical Imaging (San Diego, CA, May 2, 2002) vol 4682 pp 580

Arfelli F 2000 Synchrotron light and imaging systems for medical radiology Nuclear Instruments \& Methods in Physics Research Section a-Accelerators Spectrometers Detectors and Associated Equipment 454 11-25

Arfelli F, Assante M, Bonvicini V, Bravin A, Cantatore G, Castelli E, Dalla Palma L, Di Michiel M, Longo R, Olivo A, Pani S, Pontoni D, Poropat P, Prest M, Rashevsky A, Tromba G, Vacchi A, Vallazza E \& Zanconati F 1998a Low-dose phase contrast Xray medical imaging Phys Med Biol 43 2845-52

Arfelli F, Barbiellini G, Bonvicini V, Bravin A, Cantatore G, Castelli E, Dallapalma L, Dimichiel M, Longo R, Olivo A, Pani S, Pontoni D, Poropat P, Prest M, Rosei R, Sessa M, Tromba G \& Vacchi A 1996 Digital mammography at the trieste synchrotron light source Ieee Transactions on Nuclear Science 43 2061-2067

Arfelli F, Bonvicini V, Bravin A, Cantatore G, Castelli E, Dalla Palma L, Di Michiel M, Longo R, Olivo A, Pani S, Pontoni D, Poropat P, Prest M, Rashevsky A, Tromba G \& Vacchi A 1998b Mammography of a phantom and breast tissue with synchrotron radiation and a linear-array silicon detector Radiology 208 709-15

Arfelli F, Bonvicini V, Bravin A, Cantatore G, Castelli E, Palma L D, Michiel M D, Fabrizioli M, Longo R, Menk R H, Olivo A, Pani S, Pontoni D, Poropat P, Prest M, Rashevsky A, Ratti M, Rigon L, Tromba G, Vacchi A, Vallazza E \& Zanconati F 2000 Mammography with synchrotron radiation: phase-detection techniques Radiology 215 286-93

Arfelli F, Bravin A, Barbiellini G, Cantatore G, Castelli E, Michiel M D, Poropat P, Rosei R, Sessa M, Vacchi A, Palma L D, Longo R, Bernstorff S, Savoia A \& Tromba G 1995 Digital mammography with synchrotron radiation Rev. Sci. Instum. 66 1325-1328

Asaga T, Chiyasu S, Mastuda S, Mastuura H, Kato H, Ishida M \& Komaki T 1987 Breast imaging: dual-energy projection radiography with digital radiography Radiology 164 869-70

Asaga T, Masuzawa C, Yoshida A \& Matsuura H 1995 Dual-energy subtraction mammography J Digit Imaging 8 70-3

Badea C. 2000. Volume imaging using a combined Cone Beam CT-DTS approach. Ph.D. dissertation, University of Patras, Greece.

Badea C, Bliznakova K, Kolitsi Z \& Pallikarakis N 2002 Noise Removal in Tomosynthetic Mammographic Imaging Proceedings of the EMBEC Conference

Badea C, Kolitsi Z \& Pallikarakis N 1998 A wavelet-based method for removal of out-ofplane structures in digital tomosynthesis Comput Med Imaging Graph 22 309-15

Badea C, Kolitsi Z \& Pallikarakis N 2001 Image quality in extended arc filtered digital tomosynthesis Acta Radiol 42 244-8

Baker J A \& Lo J Y 2011 Breast tomosynthesis: state-of-the-art and review of the literature Acad Radiol 18 1298-310 
Baldelli P, Taibi A, Tuffanelli A, Gilardoni M C \& Gambaccini M 2005 A prototype of a quasi-monochromatic system for mammography applications Phys Med Biol $\mathbf{5 0}$ 2225-40

Bannister L H, Berry M M, Collins P, Dyson M \& Dussek J E 1995 Gray's Anatomy 38th ed. New York, USA: Churchill Livingstone pp. 417-424

Bassett L W 2000 Digital and Computer-Aided Mammography Breast J 6 291-293

Becher H, Schluter M, Mathey D G, Bleifeld W, Klotz E, Haaker P, Linde R \& Weiss H 1985 Coronary angiography with flashing tomosynthesis Eur Heart J 6 399-408

Bernhardt P, Mertelmeier T \& Hoheisel M 2006 X-ray spectrum optimization of full-field digital mammography: simulation and phantom study Med Phys 33 4337-49

Bertolini M, Nitrosi A, Borasi G, Botti A, Tassoni D, Sghedoni R \& Zuccoli G 2011 Contrast detail phantom comparison on a commercially available unit. Digital breast tomosynthesis (DBT) versus full-field digital mammography (FFDM) $J$ Digit Imaging 24 58-65

Breast Imaging Reporting and Data System (BI-RADS) Breast Imaging Atlas 2003 Reston, VA, American College of Radiology

Bliznakova K. 2003. Study and development of software simulation for X-ray imaging. Ph.D. dissertation, University of Patras, Greece.

Bliznakova K, Bliznakov Z \& Buliev I 2012a Comparison of algorithms for out-of-plane artifacts removal in digital tomosynthesis reconstructions Comput Methods Programs Biomed 107 75-83

Bliznakova K, Kolitsi Z \& Pallikarakis N 2005 Voxel-based Monte Carlo Radiotherapy Simulator 3rd European Medical and Biological Engineering Conference EMBEC IFMBE (Prague)

Bliznakova K, Kolitsi Z \& Pallikarakis N 2006 Dual-energy mammography: simulation studies Phys Med Biol 51 4497-515

Bliznakova K, Kolitsi Z, Speller R D, Horrocks J A, Tromba G \& Pallikarakis N 2010a Evaluation of digital breast tomosynthesis reconstruction algorithms using synchrotron radiation in standard geometry Med Phys 37 1893-903

Bliznakova K, Sechopoulos I, Buliev I \& Pallikarakis N 2012b BreastSimulator: A software platform for breast x-ray imaging research Journal of Biomedical Graphics and Computing 2 1-14

Bliznakova K, Speller R, Horrocks J, Liaparinos P, Kolitsi Z \& Pallikarakis N 2010b Experimental validation of a radiographic simulation code using breast phantom for X-ray imaging Comput Biol Med 40 208-14

Boone J M 1991 Color mammography. Image generation and receiver operating characteristic evaluation Invest Radiol 26 521-7

Boone J M 1998 Spectral modeling and compilation of quantum fluence in radiography and mammography Proc. SPIE, Medical Imaging 1998: Physics of Medical Imaging (San Diego, July 24) vol 3336 pp 592-601

Boone J M 2002 Normalized glandular dose (DgN) coefficients for arbitrary X-ray spectra in mammography: computer-fit values of Monte Carlo derived data Med Phys 29 86975

Boone J M, Fewell T R \& Jennings R J 1997 Molybdenum, rhodium, and tungsten anode spectral models using interpolating polynomials with application to mammography Med Phys 24 1863-74

Borg M, Badr I \& Royle G J 2012 The use of a figure-of-merit (FOM) for optimisation in digital mammography: a literature review Radiat Prot Dosimetry 151 81-8

Bovi M, Laitano R F, Pimpinella M, Toni M P, Casarin K, Quail E, Tromba G, Vacotto A \& Dreossi D 2007 Absolute air-kerma measurement in a synchrotron light beam by ionization free-air chamber Workshop on Absorbed Dose and Air Kerma Primary Standards

Boyd N F, Lockwood G A, Martin L J, Knight J A, Byng J W, Yaffe M J \& Tritchler D L 1998 Mammographic densities and breast cancer risk Breast Dis 10 113-26 
Boyd N F, Martin L J, Bronskill M, Yaffe M J, Duric N \& Minkin S 2010 Breast tissue composition and susceptibility to breast cancer J Natl Cancer Inst 102 1224-37

Brettle D S \& Cowen A R 1994 Dual-energy digital mammography utilizing stimulated phosphor computed radiography Phys Med Biol 39 1989-2004

Buliev I. 2004. Simulation and design of a C-arm based tomographic system for heart imaging. Ph.D. dissertation, University of Patras, Greece.

Burattini E, Cossu E, Di Maggio C, Gambaccini M, Indovina P L, Marziani M, Pocek M, Simeoni S \& Simonetti G 1995 Mammography with synchrotron radiation Radiology 195 239-44

Burattini E, Gambaccini M, Indovina P L, Marziani M, Simeoni S \& Taibi A 1994 Dose and image-quality evaluation in synchrotron radiation mammography Eur. Radiol. 4 464469

Carton A K, Gavenonis S C, Currivan J A, Conant E F, Schnall M D \& Maidment A D 2010 Dual-energy contrast-enhanced digital breast tomosynthesis--a feasibility study $\mathrm{Br} J$ Radiol 83 344-50

Castelli E, Tonutti M, Arfelli F, Longo R, Quaia E, Rigon L, Sanabor D, Zanconati F, Dreossi D, Abrami A, Quai E, Bregant P, Casarin K, Chenda V, Menk R H, Rokvic T, Vascotto A, Tromba G \& Cova M A 2011 Mammography with synchrotron radiation: first clinical experience with phase-detection technique Radiology 259 684-94

Chakraborty D P \& Barnes G T 1989 An energy sensitive cassette for dual-energy mammography Med Phys 16 7-13

Chan H P, Sahiner B, Petrick N, Hadjiiski L \& Paquerault S 2005 Computer-Aided Diagnosis of Breast Cancer: In Medical Image Analysis Methods, Costaridou L. (Ed.), CRC Press, Taylor \& Francis Group, Boca Raton, FL, US

Chawla A S, Lo J Y, Baker J A \& Samei E 2009 Optimized image acquisition for breast tomosynthesis in projection and reconstruction space Med Phys 36 4859-69

Chawla A S, Samei E, Saunders R S, Lo J Y \& Baker J A 2008 A mathematical model platform for optimizing a multiprojection breast imaging system Med Phys 35 133745

Chen R C, Longo R, Rigon L, Zanconati F, De Pellegrin A, Arfelli F, Dreossi D, Menk R H, Vallazza E, Xiao T Q \& Castelli E 2010 Measurement of the linear attenuation coefficients of breast tissues by synchrotron radiation computed tomography Phys Med Biol 55 4993-5005

Ciatto S, Houssami N, Bernardi D, Caumo F, Pellegrini M, Brunelli S, Tuttobene P, Bricolo P, Fanto C, Valentini M, Montemezzi S \& Macaskill P 2013 Integration of 3D digital mammography with tomosynthesis for population breast-cancer screening (STORM): a prospective comparison study Lancet Oncol 14 583-9

Colsher J G 1977 Iterative three-dimensional image reconstruction from tomographic projections Comput. Graph. Image Process. 6 513-537

Cooper A P 1840 Anatomy of the Breast London, UK: Longman, Orme, Green, Browne and Longmans

Costaridou L, Skiadopoulos S, Karahaliou A, Arikidis A \& Panayiotakis G 2008 ComputerAided Diagnosis in Breast Imaging: Trends and Challenges: In Handbook of Research on Advanced Techniques in Diagnostic Imaging and Biomedical Applications T. P. Exarchos, A. Papadopoulos, D .I. Fotiadis (Eds), IDEA Group Inc. Global.

Cunningham L 1977 The anatomy of the arteries and veins of the breast $J$ Surg Oncol 9 71-85

Dance D R 1990 Monte Carlo calculation of conversion factors for the estimation of mean glandular breast dose Phys Med Biol 35 1211-9

Dance D R, Skinner C L, Young K C, Beckett J R \& Kotre C J 2000a Additional factors for the estimation of mean glandular breast dose using the UK mammography dosimetry protocol Phys Med Biol 45 3225-40

Dance D R, Thilander A K, Sandborg M, Skinner C L, Castellano I A \& Carlsson G A 2000b Influence of anode/filter material and tube potential on contrast, signal-to-noise ratio 
and average absorbed dose in mammography: a Monte Carlo study $\mathrm{Br} \mathrm{J}$ Radiol 73 1056-67

Dance D R, Young K C \& Van Engen R E 2009 Further factors for the estimation of mean glandular dose using the United Kingdom, European and IAEA breast dosimetry protocols Phys Med Biol 54 4361-72

Dance D R, Young K C \& Van Engen R E 2011 Estimation of mean glandular dose for breast tomosynthesis: factors for use with the UK, European and IAEA breast dosimetry protocols Phys Med Biol 56 453-71

Diekmann F, Diekmann S, Richter K, Bick U, Fischer T, Lawaczeck R, Press W R, Schon K, Weinmann H J, Arkadiev V, Bjeoumikhov A, Langhoff N, Rabe J, Roth P, Tilgner J, Wedell R, Krumrey M, Linke U, Ulm G \& Hamm B 2004 Near monochromatic Xrays for digital slot-scan mammography: initial findings Eur Radiol 14 1641-6

Dobbins J T 1990 Matrix Inversion Tomosynthesis improvements in longitudinal x-ray slice imaging U.S. patent \# 4,903,204 Duke University

Dobbins J T, 3rd 2009 Tomosynthesis imaging: at a translational crossroads Med Phys $\mathbf{3 6}$ 1956-67

Dobbins J T, 3rd \& Godfrey D J 2003 Digital x-ray tomosynthesis: current state of the art and clinical potential Phys Med Biol 48 R65-106

Dobbins J T, Powell A O \& Weaver Y K 1987 Matrix inversion tomosynthesis: initial image reconstruction RSNA 73rd Scientific Assembly (Chicago, IL)

Dreossi D, Abrami A, Arfelli F, Bregant P, Casarin K, Chenda V, Cova M A, Longo R, Menk R H, Quai E, Quaia E, Rigon L, Rokvic T, Sanabor D, Tonutti M, Tromba G, Vascotto A, Zanconati F \& Castelli E 2008 The mammography project at the SYRMEP beamline Eur J Radiol 68 S58-62

Dreossi D, Bergamaschi A, Schmitt B, Vallazza E, Arfelli F, Longo R, Menk R H, Rigon L, Rokvic T, Venanzi C \& Castelli E 2007 Clinical mammography at the SYRMEP beam line: Toward the digital detection system Nucl Instrum Methods Phys Res A 576 160-163

Edholm P, Granlund G, Knutsson H \& Petersson C 1980 Ectomography. A new radiographic method for reproducing a selected slice of varying thickness Acta Radiol Diagn (Stockh) 21 433-42

Edholm P \& Quiding L 1970 Elimination of blur in linear tomography Acta Radiol Diagn (Stockh) 10 441-7

Elter M \& Horsch A 2009 CADx of mammographic masses and clustered microcalcifications: a review Med Phys 36 2052-68

Engelke W, Ruttimann U E, Tsuchimochi M \& Bacher J D 1992 An experimental study of new diagnostic methods for the examination of osseous lesions in the temporomandibular joint Oral Surg Oral Med Oral Pathol 73 348-59

Eriksson M 1988 Synchrotron Radiation News 1

Fahrig R \& Yaffe M J 1994 Optimization of spectral shape in digital mammography: dependence on anode material, breast thickness, and lesion type Med Phys 21 147381

Fedon C, Quai E, Arfelli F, Dreossi D, Rigon L, Tonutti M, Tromba G, Cova M \& Longo R 2014 Phase-Contrast Mammography with Synchrotron Radiation: Dosimetric Results [Online] Scientific Exhibit ECR 2014 Available at: http://dx.doi.org/10.1594/ecr2014/C-1253

Feng S S \& Sechopoulos I 2012 Clinical digital breast tomosynthesis system: dosimetric characterization Radiology 263 35-42

Fitousi N T, Delis H \& Panayiotakis G 2012 Monte Carlo simulation of breast imaging using synchrotron radiation Med Phys 39 2069-77

Gambaccini M, Tuffanelli A, Taibi A \& Del Guerra A 2001 Spatial resolution measurements in quasimonochromatic $\mathrm{x}$ rays with mosaic crystals for mammography application Med Phys 28 412-8

Garrison J B, Grant D G, Guier W H \& Johns R J 1969 Three dimensional roentgenography Am J Roentgenol Radium Ther Nucl Med 105 903-8 
Ghosh Roy D N, Kruger R A, Yih B \& Del Rio P 1985 Selective plane removal in limited angle tomographic imaging Med Phys 12 65-70

Gifford H C, Didier C S, Das M \& Glick S J 2008 Optimizing breast-tomosynthesis acquisition parameters with scanning model observers vol 6917 pp 69170S-69170S-9

Glick S J \& Gong X 2006 Optimal spectra for indirect detector breast tomosynthesis vol 6142 pp 61421L-61421L-9

Godfrey D J, Mcadams H P \& Dobbins J T, 3rd 2006 Optimization of the matrix inversion tomosynthesis (MITS) impulse response and modulation transfer function characteristics for chest imaging Med Phys 33 655-67

Godfrey D J, Mcadams H P \& Dobbins J T, 3rd 2013 The effect of averaging adjacent planes for artifact reduction in matrix inversion tomosynthesis Med Phys $\mathbf{4 0} 021907$

Gong X, Glick S J, Liu B, Vedula A A \& Thacker S 2006 A computer simulation study comparing lesion detection accuracy with digital mammography, breast tomosynthesis, and cone-beam CT breast imaging Med Phys 33 1041-52

Grant D G 1972 Tomosynthesis: a three-dimensional radiographic imaging technique IEEE Trans Biomed Eng 19 20-8

Gur D, Abrams G S, Chough D M, Ganott M A, Hakim C M, Perrin R L, Rathfon G Y, Sumkin J H, Zuley M L \& Bandos A I 2009 Digital breast tomosynthesis: observer performance study AJR Am J Roentgenol 193 586-91

Haaker P, Klotz E, Koppe R, Linde R \& Mathey D G 1985a First clinical results with digital flashing tomosynthesis in coronary angiography Eur Heart $J 6$ 913-20

Haaker P, Klotz E, Koppe R, Linde R \& Moller H 1985b A new digital tomosynthesis method with less artifacts for angiography Med Phys 12 431-6

Haas B M, Kalra V, Geisel J, Raghu M, Durand M \& Philpotts L E 2013 Comparison of tomosynthesis plus digital mammography and digital mammography alone for breast cancer screening Radiology 269 694-700

Hammerstein G R, Miller D W, White D R, Masterson M E, Woodard H Q \& Laughlin J S 1979 Absorbed radiation dose in mammography Radiology 130 485-91

Hoheisel M, Bernhardt P, Lawaczeck R \& Pietsch H 2006 Comparison of polychromatic and monochromatic X-rays for imaging vol 6142 pp 614209-614209-8

Hoheisel M, Lawaczeck R, Pietsch H \& Arkadiev V 2005 Advantages of monochromatic xrays for imaging vol 5745 pp 1087-1095

Johns P C, Drost D J, Yaffe M J \& Fenster A 1985 Dual-energy mammography: initial experimental results Med Phys 12 297-304

Johns P C \& Yaffe M J 1985 Theoretical optimization of dual-energy x-ray imaging with application to mammography Med Phys 12 289-96

Johnston R E, Washburn D, Pisano E, Burns C, Thomlinson W C, Chapman L D, Arfelli F, Gmur N F, Zhong Z \& Sayers D 1996 Mammographic phantom studies with synchrotron radiation Radiology 200 659-63

Kamarianakis Z, Buliev I \& Pallikarakis N 2011 Robust identification and localization of intramedullary nail holes for distal locking using CBCT: a simulation study Med Eng Phys 33 479-89

Kamarianakis Z, Soimu D, Bliznakova K \& Pallikarakis N 2009 Microcalcification Detection using Digital Tomosynthesis, Dual Energy Mammography and Cone Beam Computed Tomography: A Comparative Study In: 4th European Conference of the International Federation for Medical and Biological Engineering Sloten, J., Verdonck, P., Nyssen, M. \& Haueisen, J. (eds.) Springer Berlin Heidelberg, pp. 660663

Kampp T D 1986 The backprojection method applied to classical tomography Med Phys $\mathbf{1 3}$ 329-33

Karahaliou A, Arikidis A, Skiadopoulos S, Panayiotakis G \& Costaridou L 2012 Computerized Image Analysis of Mammographic Microcalcifications: Diagnosis and Prognosis: Chapter 16, in Mammography - Recent Advances, (Eds) Nachiko Uchiyama and Marcelo Zanchetta do Nascimento 
Knutsson H E, Edholm P, Granlund G H \& Petersson C U 1980 Ectomography--a new radiographic reconstruction method--I. Theory and error estimates IEEE Trans Biomed Eng 27 640-8

Koch E E, Eastman D E \& Farges Y (eds.) 1983 Handbook of Synchrotron Radiation, Vol.1a, Chapter 1 North-Holland Publishing Company, Amsterdam

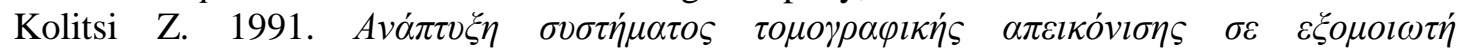
$\alpha \kappa \tau \imath v o \theta \varepsilon \rho \alpha \pi \varepsilon i \alpha \varsigma$. Ph.D. dissertation, University of Patras, Greece.

Kolitsi Z, Panayiotakis G, Anastassopoulos V, Scodras A \& Pallikarakis N 1992 A multiple projection method for digital tomosynthesis Med Phys 19 1045-50

Kolitsi Z, Panayiotakis G \& Pallikarakis N 1993 A method for selective removal of out-ofplane structures in digital tomosynthesis Med Phys 20 47-50

Lazos D, Bliznakova K, Kolitsi Z \& Pallikarakis N 2003a An integrated research tool for Xray imaging simulation Comput Methods Programs Biomed 70 241-51

Lemacks M R, Kappadath S C, Shaw C C, Liu X \& Whitman G J 2002 A dual-energy subtraction technique for microcalcification imaging in digital mammography--a signal-to-noise analysis Med Phys 29 1739-51

Lewin J M, Isaacs P K, Vance V \& Larke F J 2003 Dual-energy contrast-enhanced digital subtraction mammography: feasibility Radiology 229 261-8

Li T, Sun L, Miller N, Nicklee T, Woo J, Hulse-Smith L, Tsao M S, Khokha R, Martin L \& Boyd N 2005 The association of measured breast tissue characteristics with mammographic density and other risk factors for breast cancer Cancer Epidemiol Biomarkers Prev 14 343-9

Longo R, Tonutti M, Rigon L, Arfelli F, Dreossi D, Quai E, Zanconati F, Castelli E, Tromba G \& Cova M A 2014 Clinical study in phase- contrast mammography: image-quality analysis Philos Trans A Math Phys Eng Sci 37220130025

Lu Y, Chan H P, Wei J, Goodsitt M, Carson P L, Hadjiiski L, Schmitz A, Eberhard J W \& Claus B E 2011 Image quality of microcalcifications in digital breast tomosynthesis: effects of projection-view distributions Med Phys 38 5703-12

Lu Y, Chan H P, Wei J \& Hadjiiski L M 2010 Selective-diffusion regularization for enhancement of microcalcifications in digital breast tomosynthesis reconstruction Med Phys 37 6003-14

Malliori A, Bliznakova K, Daskalaki A \& Pallikarakis N 2014 Graphical User Interface for Breast Tomosynthesis Reconstructions: An application using Anisotropic Diffusion Filtering IFMBE Proc. XIII Mediterranean Conference on Medical and Biological Engineering and Computing-MEDICON (Seville, Spain, September 25-28, 2013) vol 41 pp 479-482

Malliori A, Bliznakova K, Dermitzakis A \& Pallikarakis N 2013 Evaluation of the Effect of Acquisition Parameters on Image Quality in Digital Breast Tomosynthesis: Simulation Studies IFMBE Proc. Congress on Medical Physics and Biomedical Engineering - WC2012 (Beijing, China, May 26-31, 2012) vol 39 pp 2211-2214

Malliori A, Bliznakova K \& Pallikarakis N 2010a A computer-based platform for Digital breast Tomosynthesis simulation studies IEEE/EMBS Region 8 International Conference on Information Technology Applications in Biomedicine, ITAB (Corfu, November 2-5, 2010)

Malliori A, Bliznakova K, Speller R D, Horrocks J A, Rigon L, Tromba G \& Pallikarakis N 2012 Image quality evaluation of breast tomosynthesis with synchrotron radiation Med Phys 39 5621-34

Maravilla K R, Murry R C, Jr. \& Horner S 1983 Digital tomosynthesis: technique for electronic reconstructive tomography AJR Am J Roentgenol 141 497-502

Margaritondo G, Hwu Y \& Tromba G 2003 Synchrotron light: From basics to coherencerelated applications In: Synchrotron Radiation: Fundamentals, Methodologies and Applications Mobilio, S. \& Vlaic, G. (eds.) Italian Physical Society, Bologna pp. 1-23

Matsuo H, Iwata A, Horiba I \& Suzumura N 1993 Three-dimensional image reconstruction by digital tomo-synthesis using inverse filtering IEEE Trans Med Imaging 12 307-13 
Mckinley R L, Tornai M P, Samei E \& Bradshaw M L 2004 Simulation study of a quasimonochromatic beam for x-ray computed mammotomography Med Phys 31 800-13

Mckinley R L, Tornai M P, Samei E \& Bradshaw M L 2005 Initial study of quasimonochromatic X-ray beam performance for X-ray computed mammotomography Nuclear Science, IEEE Transactions on 52 1243-1250

Mertelmeier T, Ludwig J, Zhao B \& Zhao W 2008 Optimization of Tomosynthesis Acquisition Parameters: Angular Range and Number of Projections In: Digital Mammography Krupinski, E. (eds.) Springer Berlin Heidelberg, pp. 220-227

Mertelmeier T, Orman J, Haerer W \& Dudam M K 2006 Optimizing filtered backprojection reconstruction for a breast tomosynthesis prototype device vol 6142 pp $61420 \mathrm{~F}-$ 61420F-12

Messaris G, Kolitsi Z, Badea C \& Pallikarakis N 1999 Three-dimensional localisation based on projectional and tomographic image correlation: an application for digital tomosynthesis Med Eng Phys 21 101-9

Michelson A 1927 Studies in Optics U. of Chicago Press

Miller E R, Mccurry E M \& Hruska B 1971 An infinite number of laminagrams from a finite number of radiographs Radiology $\mathbf{9 8}$ 249-55

Mobilio S \& Balerna A 2003 Introduction to the main properties of Synchrotron Radiation In: Synchrotron Radiation: Fundamentals, Methodologies and Applications Mobilio, S. \& Vlaic, G. (eds.) Italian Physical Society, Bologna pp. 1-23

Moeckli R, Verdun F R, Fiedler S, Pachoud M, Schnyder P \& Valley J F 2000 Objective comparison of image quality and dose between conventional and synchrotron radiation mammography Phys Med Biol 45 3509-23

Muller B, Schlattl H, Gruner F \& Hoeschen C 2011 A laser-driven undulator x-ray source: simulation of image formation and dose deposition in mammography Medical Imaging 2011: Physics of Medical Imaging 7961

Nadjmi M, Weiss H, Klotz E \& Linde R 1980 Flashing tomosynthesis--a new tomographic method Neuroradiology 19 113-7

Netter F H 2006 Atlas of Human Anatomy 4th ed.: Saunders

Niklason L T, Christian B T, Niklason L E, Kopans D B, Castleberry D E, Opsahl-Ong B H, Landberg C E, Slanetz P J, Giardino A A, Moore R, Albagli D, Dejule M C, Fitzgerald P F, Fobare D F, Giambattista B W, Kwasnick R F, Liu J, Lubowski S J, Possin G E, Richotte J F, Wei C Y \& Wirth R F 1997 Digital tomosynthesis in breast imaging Radiology 205 399-406

Orman J, Mertelmeier T \& Haerer W 2006 Adaptation of Image Quality Using Various Filter Setups in the Filtered Backprojection Approach for Digital Breast Tomosynthesis In: Digital Mammography Astley, S., Brady, M., Rose, C. \& Zwiggelaar, R. (eds.) Springer Berlin Heidelberg, pp. 175-182

Paap E, Holland R, Den Heeten G J, Van Schoor G, Botterweck A A, Verbeek A L \& Broeders M J 2010 A remarkable reduction of breast cancer deaths in screened versus unscreened women: a case-referent study Cancer Causes Control 21 1569-73

Paci E 2012 Summary of the evidence of breast cancer service screening outcomes in Europe and first estimate of the benefit and harm balance sheet J Med Screen 19 Suppl 1 513

Pani S, Longo R, Dreossi D, Montanari F, Olivo A, Arfelli F, Bergamaschi A, Poropat P, Rigon L, Zanconati F, Dalla Palma L \& Castelli E 2004 Breast tomography with synchrotron radiation: preliminary results Phys Med Biol 49 1739-54

Perona P \& Malik J 1987 Scale Space and Edge Detection Using Anisotropic Diffusion Proc. IEEE Comp. Soc. Workshop on Computer Vision IEEE Computer Society Press, Washington (Miami Beach)

Perry N, Broeders M, Wolf C, Törnberg S, Holland R \& Karsa L (eds.) 2006 European guidelines for quality assurance in breast cancer screening and diagnosis 4th ed., Office for Official Publications of the European Communities, Luxembourg 
Petersson C U, Edholm P, Granlund G H \& Knutsson H E 1980 Ectomography--a new radiographic reconstruction method--II. Computer simulated experiments IEEE Trans Biomed Eng 27 649-55

Poplack S P, Tosteson T D, Kogel C A \& Nagy H M 2007 Digital breast tomosynthesis: initial experience in 98 women with abnormal digital screening mammography AJR Am J Roentgenol 189 616-23

Quai E, Longo R, Zanconati F, Jaconelli G, Tonutti M, Abrami A, Arfelli F, Dreossi D, Tromba G \& Cova M A 2013 First application of computed radiology to mammography with synchrotron radiation Radiol Med 118 89-100

Reiser I \& Nishikawa R M 2010 Task-based assessment of breast tomosynthesis: effect of acquisition parameters and quantum noise Med Phys 37 1591-600

Ren B R, Wu T, Smith A, Ruth C, Niklason L, Jing Z X \& Stein J 2006 The Dependence of Tomosynthesis Imaging Performance on the Number of Scan Projections Lecture Notes in Computer Science (Springer, Berlin, 2006) vol 4046 pp 517-524

Rigon L 2008 Clinical Mammography With Synchrotron Radiation And The New Digital Development [Online] Proc. of the ICFA Workshop on Compton Sources for X/gamma Rays: Physics and Applications, 7-12 September, Alghero (Sardinia), Italy Available

at: https://agenda.infn.it/getFile.py/access?contribId=4\&sessionId=12\&resId=0\&materia IId=slides\&confId $=367$

Rimkus D S, Gill B M, Baily N A, Talner L B \& Friedman P J 1989 Digital tomosynthesis: phantom and patient studies with a prototype unit Comput Med Imaging Graph 13 $307-18$

Rose A 1948 The sensitivity performance of the human eye on an absolute scale $J$ Opt Soc Am 38 196-208

Rose S L, Tidwell A L, Bujnoch L J, Kushwaha A C, Nordmann A S \& Sexton R, Jr. 2013 Implementation of breast tomosynthesis in a routine screening practice: an observational study AJR Am J Roentgenol 200 1401-8

Ruttimann U E, Groenhuis R A \& Webber R L 1984 Restoration of digital multiplane tomosynthesis by a constrained iteration method IEEE Trans Med Imaging 3 141-8

Samala R K, Chan H-P, Lu Y, Hadjiiski L, Wei J, Sahiner B \& Helvie M A 2014 Computeraided detection of clustered microcalcifications in multiscale bilateral filtering regularized reconstructed digital breast tomosynthesis volume Medical Physics 41 -

Samei E, Dobbins J T, 3rd, Lo J Y \& Tornai M P 2005 A framework for optimising the radiographic technique in digital X-ray imaging Radiat Prot Dosimetry 114 220-9

Sarkar V, Shi C, Rassiah-Szegedi P, Diaz A, Eng T \& Papanikolaou N 2009 The effect of a limited number of projections and reconstruction algorithms on the image quality of megavoltage digital tomosynthesis J Appl Clin Med Phys 102970

Sechopoulos I 2013a A review of breast tomosynthesis. Part I. The image acquisition process Med Phys 40014301

Sechopoulos I 2013b A review of breast tomosynthesis. Part II. Image reconstruction, processing and analysis, and advanced applications Med Phys $\mathbf{4 0} 014302$

Sechopoulos I \& Ghetti C 2009 Optimization of the acquisition geometry in digital tomosynthesis of the breast Med Phys 36 1199-207

Shaheen E, Van Ongeval C, Zanca F, Cockmartin L, Marshall N, Jacobs J, Young K C, D R D \& Bosmans H 2011 The simulation of 3D microcalcification clusters in 2D digital mammography and breast tomosynthesis Med Phys 38 6659-71

Skaane P 2009 Studies comparing screen-film mammography and full-field digital mammography in breast cancer screening: updated review Acta Radiol 50 3-14

Skaane P, Bandos A I, Gullien R, Eben E B, Ekseth U, Haakenaasen U, Izadi M, Jebsen I N, Jahr G, Krager M, Niklason L T, Hofvind S \& Gur D 2013 Comparison of digital mammography alone and digital mammography plus tomosynthesis in a populationbased screening program Radiology 267 47-56

Smith A, Niklason L, Ren B, Wu T \& Ruth C 2006 Lesion Visibility in Low Dose Tomosynthesis 4046 pp. 160-166 
Soimu D, Buliev I \& Pallikarakis N 2008 Studies on circular isocentric cone-beam trajectories for 3D image reconstructions using FDK algorithm Comput Med Imaging Graph 32 210-20

Soimu D, Kolitsi Z \& Pallikarakis N 2005 A multiple projection algorithm with noise removal of out-of-focus plane structures for digital tomosynthesis using a stationary detector 4rd European Symposium in Biomedical Engineering and Medical Physics (Patras, June 25-27) vol pp

Sone S, Kasuga T, Sakai F, Aoki J, Izuno I, Tanizaki Y, Shigeta H \& Shibata K 1991 Development of a high-resolution digital tomosynthesis system and its clinical application Radiographics 11 807-22

Sone S, Kasuga T, Sakai F, Kawai T, Oguchi K, Hirano H, Li F, Kubo K, Honda T, Haniuda M, Takemura K \& Hosoba M 1995 Image processing in the digital tomosynthesis for pulmonary imaging European Radiology 5 96-101

Sone S, Kasuga T, Sakai F, Oguchi K, Itoh A, Li F, Maruyama Y, Kubo K, Honda T, Haniuda M \& Takemura K 1996 Digital tomosynthesis imaging of the lung Radiat Med 14 53-63

Stiel G M, Stiel L G, Klotz E \& Nienaber C A 1993 Digital flashing tomosynthesis: a promising technique for angiocardiographic screening IEEE Trans Med Imaging 12 314-21

Suryanarayanan S, Karellas A, Vedantham S, Glick S J, D'orsi C J, Baker S P \& Webber R L 2000 Comparison of tomosynthesis methods used with digital mammography Acad Radiol 7 1085-97

Thilander-Klang A C, Ackerholm P H, Berlin I C, Bjurstam N G, Mattsson S L, Mansson L G, Von Scheele C \& Thunberg S J 1997 Influence of anode-filter combinations on image quality and radiation dose in 965 women undergoing mammography Radiology 203 348-54

Timberg P, Bath M, Andersson I, Mattsson S, Tingberg A \& Ruschin M 2010 In-plane visibility of lesions using breast tomosynthesis and digital mammography Med Phys 37 5618-26

Tobon H \& Salazar H 1975 Ultrastructure of the human mammary gland. II. Postpartum lactogenesis $J$ Clin Endocrinol Metab 40 834-44

Tromba G, Cova M \& Castelli E 2011 Phase-Contrast Mammography at the SYRMEP Beamline of Elettra Synchrotron Radiation News 24 3-7

Van De Sompel D, Brady S M \& Boone J 2011 Task-based performance analysis of FBP, SART and ML for digital breast tomosynthesis using signal CNR and Channelised Hotelling Observers Med Image Anal 15 53-70

Van Engen R E, Young K C, Bosmans H \& Thijsen M 2006 European protocol for the quality control of the physical and technical aspects of mammography screening. Chapter $2 \mathrm{~b}$ : Digital mammography. In: European guidelines for quality assurance in breast cancer screening and diagnosis 4th ed. Perry, N., Broeders, M., Wolf, C., Törnberg, S., Holland, R. \& Karsa, L. (eds.) European Commission, Office for Official Publications of the European Union, Luxembourg pp. 105-106

Wagner R F 1977 Toward a unified view of radiological imaging systems. Part II: Noisy images Med Phys 4 279-96

Webber R L, Horton R A, Tyndall D A \& Ludlow J B 1997 Tuned-aperture computed tomography (TACT). Theory and application for three-dimensional dento-alveolar imaging Dentomaxillofac Radiol 26 53-62

Webber R L, Underhill H R \& Freimanis R I 2000 A controlled evaluation of tuned-aperture computed tomography applied to digital spot mammography J Digit Imaging 13 90-7

Wolbarst A B 1993 Physics of radiology Norwalk, Connecticut: Appleton \& Lange

Wu T, Moore R H, Rafferty E A \& Kopans D B 2004 A comparison of reconstruction algorithms for breast tomosynthesis Med Phys 31 2636-47

Wu T, Stewart A, Stanton M, Mccauley T, Phillips W, Kopans D B, Moore R H, Eberhard J W, Opsahl-Ong B, Niklason L \& Williams M B 2003 Tomographic mammography 
using a limited number of low-dose cone-beam projection images Med Phys 30 36580

Yaffe M J 2003 Mammography In: Biomedical Imaging Mudry, K. M., Plonsey, R. \& Bronzino, J. D. (eds.) pp.

Yang X, Sechopoulos I \& Fei B 2011 Automatic Tissue Classification for High-resolution Breast CT Images Based on Bilateral Filtering Medical Imaging 2012: Image Processing 7962 79623H

Yang X, Wu S, Sechopoulos I \& Fei B 2012 Cupping Artifact Correction and Automated Classification for High-Resolution Dedicated Breast CT Images Medical Physics 39 6397-6406

Yi Y, Lai C J, Han T, Zhong Y, Shen Y, Liu X, Ge S, You Z, Wang T \& Shaw C C 2011 Radiation doses in cone-beam breast computed tomography: a Monte Carlo simulation study Med Phys 38 589-97

Yoon K H, Kwon Y M, Choi B J, Son H H, Ryu C W, Chon K S, Park S H \& Juhng S K 2012 Monochromatic X-rays for low-dose digital mammography: preliminary results Invest Radiol 47 683-7

Young S, Park S, Anderson S K, Badano A, Myers K J \& Bakic P 2009 Estimating breast tomosynthesis performance in detection tasks with variable-background phantoms Proc. SPIE, Medical Imaging 2009: Physics of Medical Imaging (Lake Buena Vista, FL, February 07, 2009) vol 7258 pp 72580O-725890

Zhang Y, Chan H P, Sahiner B, Wei J, Goodsitt M M, Hadjiiski L M, Ge J \& Zhou C 2006 A comparative study of limited-angle cone-beam reconstruction methods for breast tomosynthesis Med Phys 33 3781-95

Zhao B \& Zhao W 2008 Three-dimensional linear system analysis for breast tomosynthesis Med Phys 35 5219-32

Zhao B, Zhou J, Hu Y H, Mertelmeier T, Ludwig J \& Zhao W 2009 Experimental validation of a three-dimensional linear system model for breast tomosynthesis Med Phys $\mathbf{3 6}$ 240-51

Zhao W, Deych R \& Dolazza E 2005 Optimization of operational conditions for direct digital mammography detectors for digital tomosynthesis vol 5745 pp 1272-1281

Zhou J, Zhao B \& Zhao W 2007 A computer simulation platform for the optimization of a breast tomosynthesis system Med Phys 34 1098-109

Ziedses Des Plantes B G 1932 Eine neue methode zur differenzierung in der roentgenographie (planographie) Acta Radiol 13 182-192

Zyganitidis C, Bliznakova K \& Pallikarakis N 2007 A novel simulation algorithm for soft tissue compression Med Biol Eng Comput 45 661-669 